\title{
Visible-Light-Induced, Palladium-Catalyzed 1,4-Difunctionalization of 1,3-Dienes with Bromodifluoroacetamides
}

Zhi-Lin Liu, ${ }^{\dagger}, "$ Zhi-Peng Ye, ${ }^{\dagger}, /$ Yi-Xuan Chen, ${ }^{\dagger}$ Yu Zheng, ${ }^{\dagger}$ Zhen-Zhen Xie, ${ }^{\dagger}$ Jian-Ping Guan, ${ }^{\dagger}$ Jun-An Xiao, ${ }^{\S}$ Kai Chen ${ }^{\dagger}$ Hao-Yue Xiang, ${ }^{*},, \dagger$ Hua Yang ${ }^{*}, \dagger$ $\dagger$ College of Chemistry and Chemical Engineering, Central South University, Changsha 410083, P. R. China

${ }^{*}$ School of Chemistry and Chemical Engineering, Henan Normal University, Xinxiang 453007, Henan, P. R. China

${ }^{\S}$ Guangxi Key Laboratory of Natural Polymer Chemistry and Physics, Nanning Normal University, Nanning 530001, Guangxi, P. R. China

* Hua Yang, E-mail: hyangchem@csu.edu.cn

*Hao-Yue Xiang, E-mail: xianghaoyue@csu.edu.cn

\section{Table of contents}

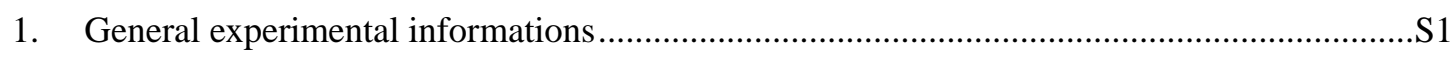

2. General procedure for the preparation of the starting materials.............................................S1

2.1 General procedure for the preparation of bromodifluoroacetamides .................................S1

2.2 General procedure for the preparation of 1,3 -dienes........................................................ 2

2.3 General procedure for the preparation of sodium sulfinates ..........................................S3

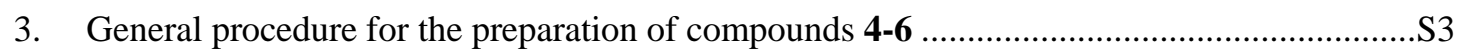

4. Scale-up reaction and unsuccessful nucleophiles ……........................................................

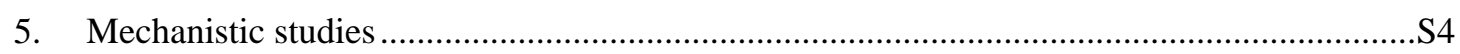

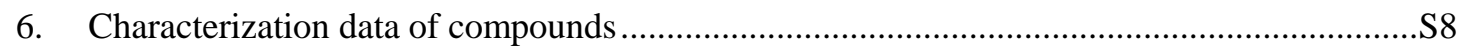

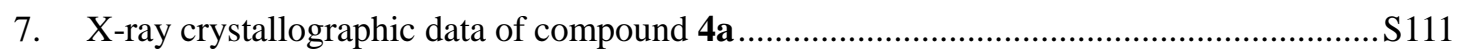




\section{General experimental informations}

Unless otherwise noted, all the reagents were purchased from commercial suppliers and used without further purification. The light source for the photocatalytic reaction is manufactured by XINNENGYUAN with power of $30 \mathrm{~W}(450 \mathrm{~nm})$, and the reaction is carried out in a borosilicate glass vessel without use of filters. ${ }^{1} \mathrm{H}$ NMR spectra were recorded at $400 \mathrm{MHz}$. The chemical shifts were recorded in ppm relative to tetramethylsilane and with the solvent resonance as the internal standard. Data were reported as follows: chemical shift, multiplicity $(\mathrm{s}=$ singlet, $\mathrm{d}=$ doublet, $\mathrm{t}=$ triplet, $\mathrm{q}=$ quartet, $\mathrm{p}=$ quintet, $\mathrm{m}=$ multiplet $)$, coupling constants $(\mathrm{Hz})$, integration .

${ }^{13} \mathrm{C}$ NMR data were collected at $100 \mathrm{MHz}$ with complete proton decoupling. ${ }^{19} \mathrm{~F}$ NMR data were collected at $376 \mathrm{MHz}$ with complete proton decoupling. Melting points (m.p.) were measured by Büchi 510 melting point apparatus and uncorrected. Infrared spectra (IR) were measured by FT-IR apparatus. High resolution mass spectroscopy (HRMS) was recorded on TOF MS $\mathrm{ES}^{+}$mass spectrometer and acetonitrile was used to dissolve the sample. Column chromatography was carried out on silica gel (200-300 mesh).

\section{General procedure for the preparation of the starting materials}

\subsection{General procedure for the preparation of bromodifluoroacetamides ${ }^{1}$}

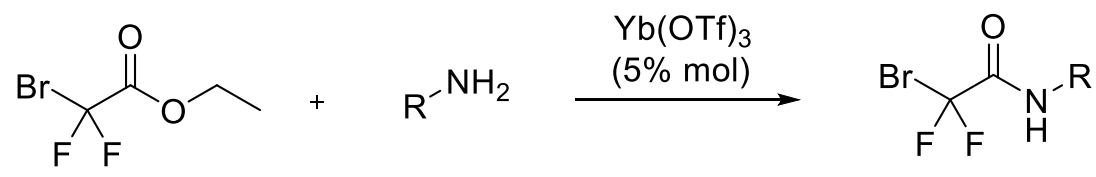

To a mixture of amines (5.0 mmol, 1.0 equiv.) and ytterbium (III) trifluoromethanesulfonate (5\% mol) was added ethyl bromodifluoroacetate $(5.0 \mathrm{mmol}, 1.0$ equiv.). The mixture was stirred until the reaction was judged to be completed by TLC analysis. The obtained crude product was purified by column chromatography (petroleum ether/EtOAc $=19: 1-9: 1)$ on silica gel to give the corresponding products. 


\subsection{General procedure for the preparation of 1,3 -dienes ${ }^{2}$}

Butadiene (2 M in THF), isoprene, 2,3-dimethyl-1,3-butadiene and 1,3-cyclohexadiene are commercially available and used as received. All other 1,3-dienes used were synthesized according to known literature procedures through Witting reaction.

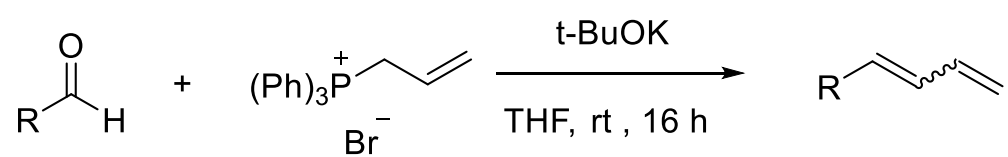

Under argon, a dry $100 \mathrm{~mL}$ round-bottom flask was charged with allyltriphenylphosphonium bromide (18 mmol, 1.2 equiv.) and dry diethyl ether $(40 \mathrm{~mL})$. To the stirred suspension, potassium tert-butoxide ( $21 \mathrm{mmol}, 1.4$ equiv.) was added at $0{ }^{\circ} \mathrm{C}$. The red reaction mixture was stirred at room temperature for 15 minutes, and then a solution of 3-phenylpropanal or cyclohexanecarbaldehyde (15 mmol, 1.0 equiv.) in dry diethyl ether $(20 \mathrm{~mL})$ was added dropwise over 15 minutes. After stirring at room temperature overnight, the reaction mixture was filtered through a short pad of silica gel and eluted with diethyl ether $(100 \mathrm{~mL} \times 3)$. The combined filtrate was concentrated under partial vacuum and the residue was purified by silica gel flash chromatograph (pentane) to give the desired product as a colorless oil in moderate yields. The cisand trans-isomers of 6-phenyl-1,3-hexadiene $(E / Z=1: 1)$ and buta-1,3-dien-1-ylcyclohexane $(E / Z$ $=1: 1)$ were inseparable by silica gel chromatography and the diastereomeric ratio of the diene was determined to be $1: 1$ by ${ }^{1} \mathrm{H}$ NMR analysis. 


\subsection{General procedure for the preparation of sodium sulfinates ${ }^{3}$}

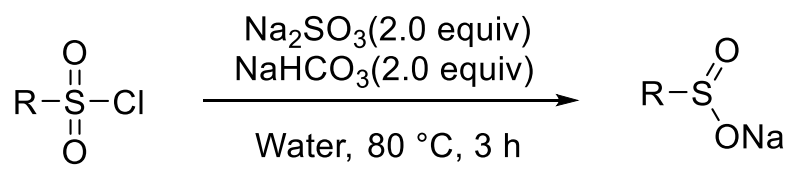

Sulfonyl chloride $(5.0 \mathrm{mmol})$ was added to a solution of sodium sulfites $(1.26 \mathrm{~g}, 10.0 \mathrm{mmol}$, 2.0 equiv.) and sodium bicarbonate $(840 \mathrm{mg}, 10.0 \mathrm{mmol}, 2.0$ equiv.) in water $(5 \mathrm{~mL}, 1 \mathrm{M})$. The mixture was heated at $80{ }^{\circ} \mathrm{C}$ (oil bath) for $3 \mathrm{~h}$. After cooling to room temperature, the volatiles were removed in vacuo. The obtained solid were repeatedly washed with ethanol. The combined ethanol washes were evaporated under reduced pressure to yield the titled sulfinates as an amorphous solid.

\section{General procedure for the preparation of compounds 4-6}

An oven-dried reaction tube containing a stirring bar was charged with the corresponding bromodifluoroacetamide 1 (0.2 mmol, 1.0 equiv.), $\mathrm{Pd}\left(\mathrm{PPh}_{3}\right)_{4}(11.5 \mathrm{mg}, 5 \mathrm{~mol} \%)$, DPEPhos (10.7 $\mathrm{mg}, 10 \mathrm{~mol} \%)$, sulfur- or nitrogen-based nucleophile ( $0.4 \mathrm{mmol}, 2.0$ equiv.), and DME ( $2 \mathrm{~mL}, 1$ M). The vessel was protected with argon by an argon balloon. Subsequently, 1,3-butadiene 2a (0.6 mmol, $0.3 \mathrm{~mL}, 2 \mathrm{M}$ in THF, 3 equiv.) or the corresponding butadiene $\mathbf{2 b - 2 \mathbf { f }}(0.4 \mathrm{mmol}, 2$ equiv.) was added. The reaction mixture was stirred at room temperature for $2 \mathrm{~h}$ or $4 \mathrm{~h}$ under irradiation of $30 \mathrm{~W}$ blue LEDs (distance app. $3 \mathrm{~cm}$ ). The solvent was removed under reduced pressure, the resulting residue was purified by flash chromatography on silica gel with petroleum ether and ethyl acetate as eluent to give products.

\section{Scale-up reaction and unsuccessful nucleophiles}

An oven-dried $120 \mathrm{~mL}$ seal tube containing a stirring bar was charged with bromodifluoroacetamide 1a (3 mmol, $0.75 \mathrm{~g}), \mathrm{Pd}\left(\mathrm{PPh}_{3}\right)_{4}(0.15 \mathrm{mmol}, 173 \mathrm{mg})$, DPEPhos $(0.3$ mmol, $161 \mathrm{mg}$ ), $\mathrm{PhSO}_{2} \mathrm{Na}(6 \mathrm{mmol}, 0.984 \mathrm{~g})$, and DME (30 mL). 1,3-butadiene $2 \mathrm{a}$ (9 mmol, 4.5 $\mathrm{mL}, 2 \mathrm{M}$ in THF, 3 equiv.) was added with a syringe. The seal tube was filled with argon gas and stirred at room temperature for $4 \mathrm{~h}$ under irradiation of $30 \mathrm{~W}$ blue LEDs (distance app. $3 \mathrm{~cm}$ ). The yellow precipitate was filtered off via a Büchner funnel and the solution was concentrated under reduced pressure and purified by flash column chromatography (petroleum ether/EtOAc $=9: 1-$ 4:1) to give the desired product $\mathbf{4 a}(0.746 \mathrm{~g}, 68 \%$ yield $)$. 


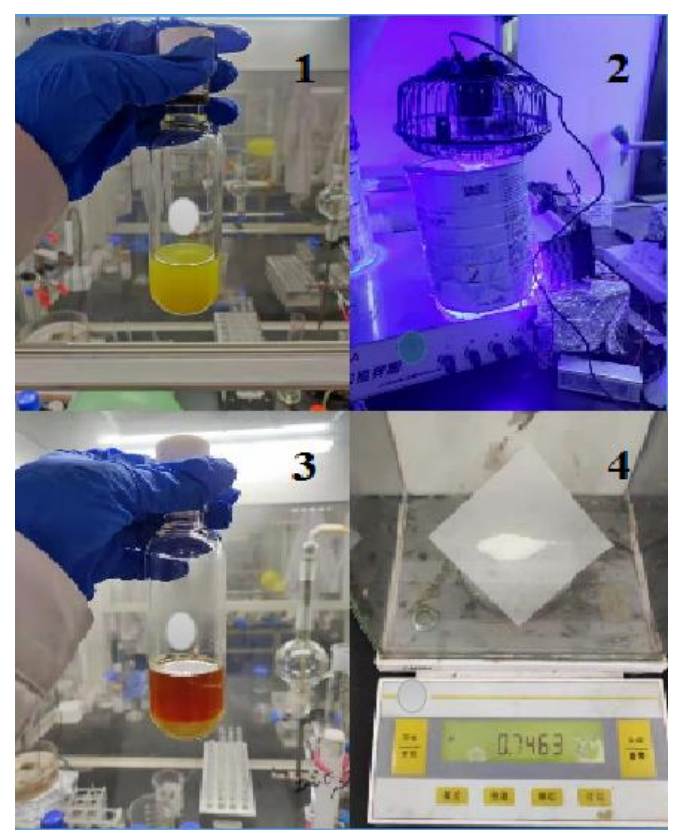

Figure S1 Scale-up synthesis of $\mathbf{4 a}$ (photographed by Z.-L. Liu)

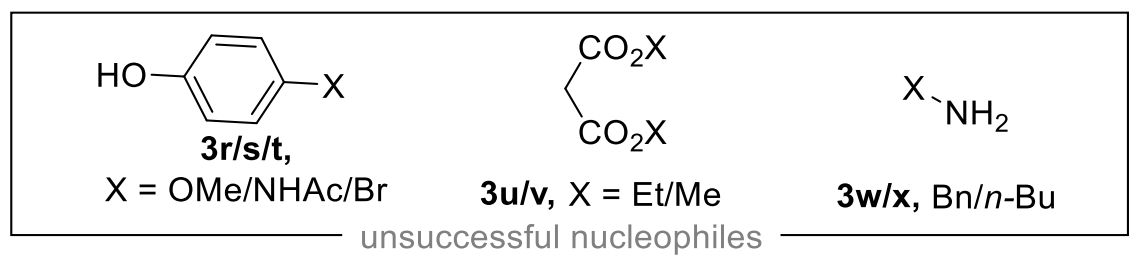

\section{Mechanistic studies}

\section{Trapping experiment}

In order to ensure whether the putative radical was trapped by TEMPO, ESI-MS analysis of the crude reaction mixture was performed. The resulting mass spectrum clearly shows a peak corresponding to the coupled product between TEMPO radical and the expected radical HRMS (ESI-TOF) m/z: [M+Na] ${ }^{+}$Calcd for 349.1698, Found 349.1708.

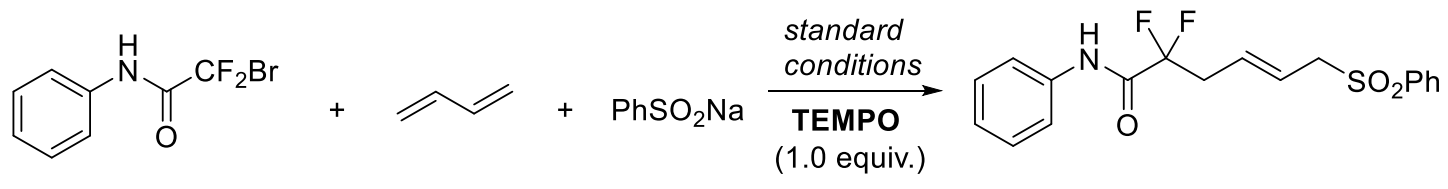

1a 


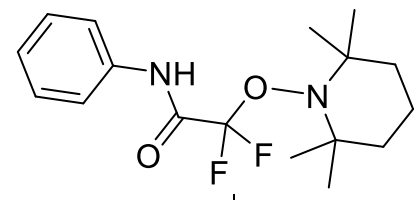

$\mathrm{Na}$

Chemical Formula: $\mathrm{C}_{17} \mathrm{H}_{24} \mathrm{~F}_{2} \mathrm{~N}_{2} \mathrm{NaO}_{2}{ }^{+}$ Exact Mass: 349.1698

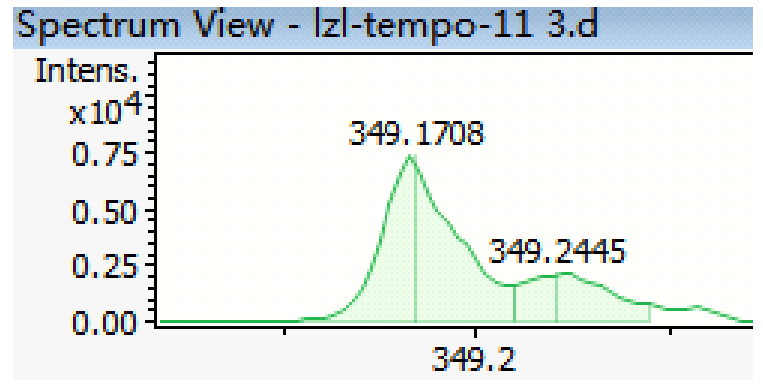

\section{Time profile of the transformation with the light on/off over time}

Six standard reactions were set up on a $0.20 \mathrm{mmol}$ scale according to the general procedure, and extra 1 equiv. of benzotrifluoride was added as the internal standard. Then the mixture was stirred and irradiated by $30 \mathrm{~W}$ blue LEDs at room temperature. After $5 \mathrm{~min}$, the blue LEDs were turned off, and one Shrek tube was removed from the irradiation setup for analysis. The remaining five Shrek tubes were stirred in the absence of light for an additional $5 \mathrm{~min}$. Then, one Shrek tube was removed for analysis, and the blue LEDs were turned back on to irradiate the remaining four reaction mixtures. After an additional 5 min of irradiation, the blue LEDs were turned off, and one Shrek tube was removed for analysis. The remaining three Shrek tubes were stirred in the absence of light for an additional $5 \mathrm{~min}$. Then, one Shrek tube was removed for analysis, and the blue LEDs were turned back on to irradiate the remaining four reaction mixtures. After $5 \mathrm{~min}$, the blue LEDs were turned off, and one Shrek tube was removed for analysis. The remaining Shrek tube was stirred in the absence of light for an additional $5 \mathrm{~min}$ and then was removed for analysis. The yield of product was determined by ${ }^{19} \mathrm{~F}$ NMR. 


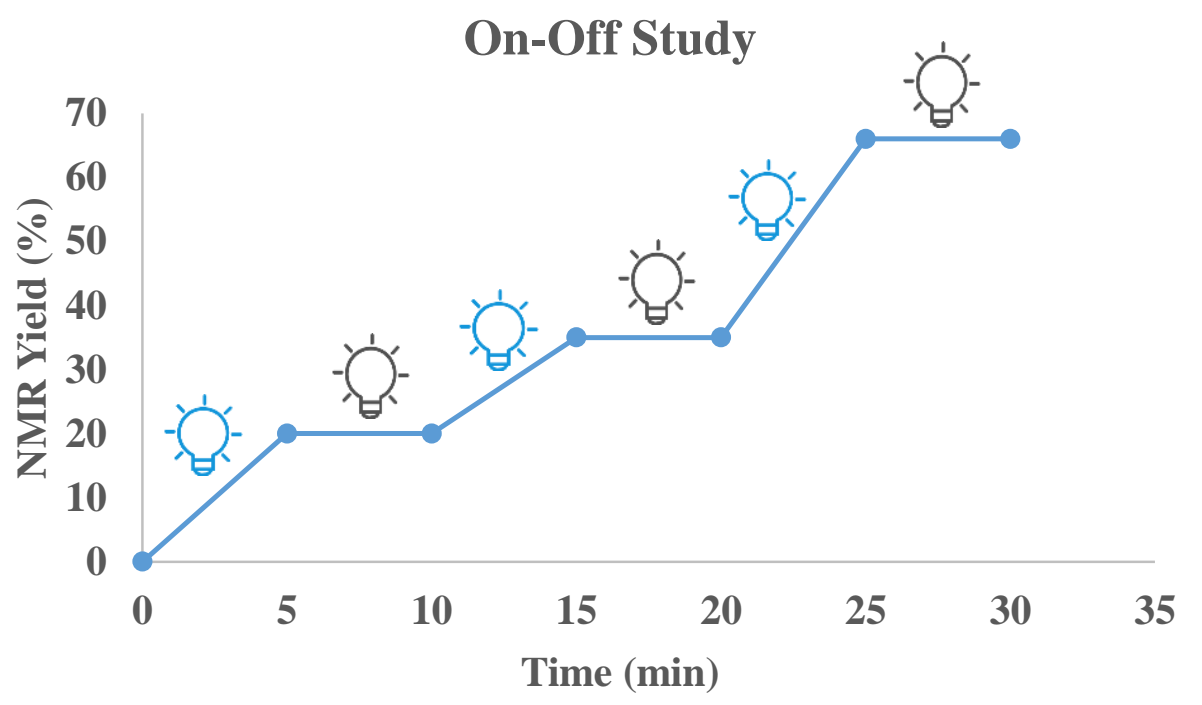

\begin{tabular}{llllllll}
\hline \multirow{2}{*}{ Entry } & \multicolumn{7}{c}{ Time (min) } \\
\cline { 2 - 7 } & 5 & 10 & 15 & 20 & 25 & 30 & Yield(\%) \\
\hline 1 & On & - & - & - & - & - & 20 \\
2 & On & Off & - & - & - & - & 20 \\
3 & On & Off & On & - & - & - & 35 \\
4 & On & Off & On & Off & - & - & 35 \\
5 & On & Off & On & Off & On & - & 66 \\
6 & On & Off & On & Off & On & Off & 66 \\
\hline
\end{tabular}




\section{References:}

1. Mai, W.-P.; Wang, F.; Zhang, X.-F.; Wang, S.-M.; Duan, Q.-P.; Lu, K. Nickel-Catalysed Radical Tandem Cyclisation/Arylation: Practical Synthesis of 4-Benzyl-3,3-Difluoro- $\gamma$-Lactams. Org. Biomol. Chem. 2018, 16, 6491-6498.

2. Wu, Q.; Hu, J.; Ren, X.; Zhou, J. An Efficient, Overall [4+1] Cycloadditon of 1,3-Dienes and Nitrene Precursors. Chem. - Eur. J. 2011, 17, 11553-11558.

3. (a) Du, B.; Qian, P.; Wang, Y.; Mei, H.; Han, J.; Pan, Y. Cu-Catalyzed Deoxygenative C2-Sulfonylation Reaction of Quinoline N-Oxides with Sodium Sulfinate. Org. Lett. 2016, 18, 4144-4147. (b) Liu, Y.; Xie, P.; Sun, Z.; Wo, X.; Gao, C.; Fu, W.; Loh, T.-P. Direct Substitution of Secondary and Tertiary Alcohols to Generate Sulfones under Catalyst- and Additive-Free Conditions. Org. Lett. 2018, 20, 5353-5356. 


\section{Characterization data of compounds}<smiles>O=C(Nc1ccccc1)C(F)(F)C/C=C/CS(=O)(=O)c1ccccc1</smiles>

4a: $59.2 \mathrm{mg}$, white solid, yield: $81 \%$; purified by flash column chromatography (PE/EA = 4/1);

m.p. $125-127^{\circ} \mathrm{C}$;

IR (neat) $v 3361,3058,2923,2851,1689,1529,1445,727,592 \mathrm{~cm}^{-1}$;

${ }^{1}$ H NMR (400 MHz, Chloroform- $d$ ) $\delta 7.98$ (s, 1H), $7.88-7.77$ (m, 2H), 7.63- 7.55(m, 3H), 7.52 (t, $J=7.6 \mathrm{~Hz}, 2 \mathrm{H}), 7.37(\mathrm{t}, J=7.9 \mathrm{~Hz}, 2 \mathrm{H}), 7.20(\mathrm{t}, J=7.4 \mathrm{~Hz}, 1 \mathrm{H}), 5.69(\mathrm{dt}, J=14.7,7.1 \mathrm{~Hz}, 1 \mathrm{H})$, $5.61-5.55(\mathrm{~m}, 1 \mathrm{H}), 3.79(\mathrm{~d}, J=7.1 \mathrm{~Hz}, 2 \mathrm{H}), 2.93(\mathrm{td}, J=16.5,6.9 \mathrm{~Hz}, 2 \mathrm{H})$;

${ }^{19} \mathbf{F}\left\{{ }^{1} \mathbf{H}\right\}$ NMR $(376 \mathrm{MHz}$, Chloroform- $d) \delta-104.87$;

${ }^{13} \mathbf{C}\left\{{ }^{1} \mathbf{H}\right\}$ NMR $(100 \mathrm{MHz}$, Chloroform- $d) \delta 161.3\left(\mathrm{t},{ }^{2} J_{C-F}=28.3 \mathrm{~Hz}\right), 138.3,135.9,133.8,129.5$ $\left(\mathrm{t},{ }^{3} J_{C-F}=5.3 \mathrm{~Hz}\right), 129.21,129.16,128.4,125.7,123.7,120.4,116.5\left(\mathrm{t},{ }^{1} J_{C-F}=256.5 \mathrm{~Hz}\right), 59.8$, $37.2\left(\mathrm{t},{ }^{2} J_{C-F}=24.4 \mathrm{~Hz}\right)$;

HRMS (ESI) m/z: $\mathrm{C}_{18} \mathrm{H}_{17} \mathrm{~F}_{2} \mathrm{NNaO}_{3} \mathrm{~S}^{+}[\mathrm{M}+\mathrm{Na}]^{+}$Calcd for 388.0789; Found 388.0795.<smiles>COc1ccccc1NC(=O)C(F)(F)C/C=C/CS(=O)(=O)c1ccccc1</smiles>

4b: $53.2 \mathrm{mg}$, yellow oil, yield: 67\%; purified by flash column chromatography (PE/EA = 4/1);

IR (neat) $v 3411,3360,3058,2923,1693,1533,1301,727 \mathrm{~cm}^{-1}$;

${ }^{1}$ H NMR $(400 \mathrm{MHz}$, Chloroform- $d$ ) $\delta 8.59(\mathrm{~s}, 1 \mathrm{H}), 8.30(\mathrm{dd}, J=8.0,1.5 \mathrm{~Hz}, 1 \mathrm{H}), 7.87-7.78(\mathrm{~m}$, 2H), $7.58(\mathrm{t}, J=7.4 \mathrm{~Hz}, 1 \mathrm{H}), 7.50(\mathrm{t}, J=7.5 \mathrm{~Hz}, 2 \mathrm{H}), 7.13(\mathrm{td}, J=8.0,1.6 \mathrm{~Hz}, 1 \mathrm{H}), 6.98(\mathrm{t}, J=$ $8.3 \mathrm{~Hz}, 1 \mathrm{H}), 6.92(\mathrm{~d}, J=9.1 \mathrm{~Hz}, 1 \mathrm{H}), 5.70(\mathrm{dt}, J=14.9,7.3 \mathrm{~Hz}, 1 \mathrm{H}), 5.55(\mathrm{dt}, J=15.3,7.1 \mathrm{~Hz}$, 1H), 3.91 (s, 3H), $3.79(\mathrm{~d}, J=7.3 \mathrm{~Hz}, 2 \mathrm{H}), 2.92(\mathrm{td}, J=16.8,7.0 \mathrm{~Hz}, 2 \mathrm{H})$;

${ }^{19} \mathbf{F}\left\{{ }^{1} \mathbf{H}\right\}$ NMR (376 MHz, Chloroform- $d$ ) $\delta-104.94$;

${ }^{13} \mathbf{C}\left\{{ }^{1} \mathbf{H}\right\}$ NMR $(100 \mathrm{MHz}$, Chloroform- $d) \delta 161.0\left(\mathrm{t},{ }^{2} J_{C-F}=28.2 \mathrm{~Hz}\right), 148.4,138.1,133.8,129.4$ $\left(\mathrm{t},{ }^{3} J_{C-F}=5.1 \mathrm{~Hz}\right), 129.1,128.4,125.7,125.4,123.6,121.1,120.0,116.6\left(\mathrm{t},{ }^{1} J_{C-F}=255.2 \mathrm{~Hz}\right)$, $110.2,59.8,55.9,37.2\left(\mathrm{t},{ }^{2} J_{C-F}=24.4 \mathrm{~Hz}\right)$;

HRMS (ESI) $\mathbf{m} / \mathbf{z}: \mathrm{C}_{19} \mathrm{H}_{19} \mathrm{~F}_{2} \mathrm{NNaO}_{4} \mathrm{~S}^{+}[\mathrm{M}+\mathrm{Na}]^{+}$Calcd for 418.0895; Found 418.0910. 
<smiles>Cc1ccccc1NC(=O)C(F)(F)C/C=C/CS(=O)(=O)c1ccccc1</smiles>

4c: $46.3 \mathrm{mg}$, yellow oil, yield: $61 \%$; purified by flash column chromatography $(\mathrm{PE} / \mathrm{EA}=4 / 1)$;

IR (neat) $v 3359,3059,2923,1692,1529,1031,1141,687 \mathrm{~cm}^{-1}$;

${ }^{1}$ H NMR (400 MHz, Chloroform- $d$ ) $\delta 7.91-7.80(\mathrm{~m}, 3 \mathrm{H}), 7.77(\mathrm{~d}, J=7.9 \mathrm{~Hz}, 1 \mathrm{H}), 7.60(\mathrm{t}, J=$ $7.4 \mathrm{~Hz}, 1 \mathrm{H}), 7.51(\mathrm{t}, J=7.6 \mathrm{~Hz}, 2 \mathrm{H}), 7.29-7.19(\mathrm{~m}, 2 \mathrm{H}), 7.15(\mathrm{t}, J=7.0 \mathrm{~Hz}, 1 \mathrm{H}), 5.69(\mathrm{dt}, J=$ 14.7, 7.1 Hz, 1H), $5.64-5.54(\mathrm{~m}, 1 \mathrm{H}), 3.79(\mathrm{~d}, J=7.1 \mathrm{~Hz}, 2 \mathrm{H}), 2.94(\mathrm{td}, J=16.7,6.9 \mathrm{~Hz}, 2 \mathrm{H})$, $2.26(\mathrm{~s}, 3 \mathrm{H})$

${ }^{19} \mathbf{F}\left\{{ }^{1} \mathbf{H}\right\}$ NMR $(376 \mathrm{MHz}$, Chloroform- $d) \delta-104.77$;

${ }^{13} \mathbf{C}\left\{{ }^{1} \mathbf{H}\right\}$ NMR $(100 \mathrm{MHz}$, Chloroform- $d) \delta 161.4\left(\mathrm{t},{ }^{2} J_{C-F}=28.1 \mathrm{~Hz}\right), 138.2,133.8,133.6,130.8$, $129.7,129.4\left(\mathrm{t},{ }^{3} J_{C-F}=5.3 \mathrm{~Hz}\right), 129.1,128.4,126.9,126.5,123.7,123.0,116.8\left(\mathrm{t},{ }^{1} J_{C-F}=255.2\right.$ $\mathrm{Hz}), 59.8,37.2\left(\mathrm{t},{ }^{2} J_{C-F}=24.4 \mathrm{~Hz}\right), 17.5$;

HRMS (ESI) $\mathbf{m} / \mathbf{z}: \mathrm{C}_{19} \mathrm{H}_{19} \mathrm{~F}_{2} \mathrm{NNaO}_{3} \mathrm{~S}^{+}[\mathrm{M}+\mathrm{Na}]^{+}$Calcd for 402.0946; Found 402.0955 .<smiles>Cc1cccc(NC(=O)C(F)(F)C/C=C/CS(=O)(=O)c2ccccc2)c1</smiles>

4d: $57.2 \mathrm{mg}$, white solid, yield: $75 \%$; purified by flash column chromatography (PE/EA = 4/1); m.p. $90-92{ }^{\circ} \mathrm{C}$;

IR (neat) v 3331, 3060, 2922, 1693, 1550, 1331, 1141, 1083, $687 \mathrm{~cm}^{-1}$;

${ }^{1}$ H NMR (400 MHz, Chloroform- $d$ ) $\delta 8.01(\mathrm{~s}, 1 \mathrm{H}), 7.88-7.76(\mathrm{~m}, 2 \mathrm{H}), 7.60(\mathrm{t}, J=7.4 \mathrm{~Hz}, 1 \mathrm{H})$, $7.51(\mathrm{t}, J=7.6 \mathrm{~Hz}, 2 \mathrm{H}), 7.41(\mathrm{~s}, 1 \mathrm{H}), 7.36(\mathrm{~d}, J=8.1 \mathrm{~Hz}, 1 \mathrm{H}), 7.23(\mathrm{t}, J=7.8 \mathrm{~Hz}, 1 \mathrm{H}), 7.00(\mathrm{~d}, J$ $=7.5 \mathrm{~Hz}, 1 \mathrm{H}), 5.68(\mathrm{dt}, J=14.8,7.2 \mathrm{~Hz}, 1 \mathrm{H}), 5.63-5.49(\mathrm{~m}, 1 \mathrm{H}), 3.78(\mathrm{~d}, J=7.2 \mathrm{~Hz}, 2 \mathrm{H}), 2.91$ $(\mathrm{td}, J=16.6,7.0 \mathrm{~Hz}, 2 \mathrm{H}), 2.34(\mathrm{~s}, 3 \mathrm{H})$;

${ }^{19} \mathbf{F}\left\{{ }^{1} \mathbf{H}\right\}$ NMR $(376 \mathrm{MHz}$, Chloroform- $d) \delta-104.87$;

${ }^{13} \mathbf{C}\left\{{ }^{1} \mathbf{H}\right\}$ NMR $(100 \mathrm{MHz}$, Chloroform- $d) \delta 161.3\left(\mathrm{t},{ }^{2} J_{C-F}=28.2 \mathrm{~Hz}\right), 139.2,138.2,135.9,133.8$, $129.5\left(\mathrm{t},{ }^{3} J_{C-F}=5.3 \mathrm{~Hz}\right), 129.2,129.0,128.4,126.5,123.6,121.0,117.5,116.6\left(\mathrm{t},{ }^{1} J_{C-F}=255.3\right.$ $\mathrm{Hz}), 59.8,37.2\left(\mathrm{t},{ }^{2} J_{C-F}=24.4 \mathrm{~Hz}\right), 21.4$;

HRMS (ESI) m/z: $\mathrm{C}_{19} \mathrm{H}_{19} \mathrm{~F}_{2} \mathrm{NNaO}_{3} \mathrm{~S}^{+}[\mathrm{M}+\mathrm{Na}]^{+}$Calcd for 402.0946; Found 402.0964 . 
$\overbrace{\mathrm{F}}^{\mathrm{N}} \overbrace{\mathrm{SO}_{2} \mathrm{Ph}}^{\mathrm{C}}$

4e: $46.5 \mathrm{mg}$, white solid, yield: $60 \%$; purified by flash column chromatography $(\mathrm{PE} / \mathrm{EA}=4 / 1)$;

m.p. $116-118^{\circ} \mathrm{C}$;

IR (neat) v 3354, 3067, 2981, 2925, 1690, 1595, 1300, 1142, 1083, $677 \mathrm{~cm}^{-1}$;

${ }^{1}$ H NMR (400 MHz, Chloroform- $d$ ) $\delta 8.17$ (s, 1H), $7.87-7.79(\mathrm{~m}, 2 \mathrm{H}), 7.62(\mathrm{t}, J=7.4 \mathrm{~Hz}, 1 \mathrm{H})$, $7.58-7.46(\mathrm{~m}, 3 \mathrm{H}), 7.38-7.20(\mathrm{~m}, 2 \mathrm{H}), 6.89(\mathrm{td}, J=7.5,6.9 \mathrm{~Hz}, 1 \mathrm{H}), 5.69(\mathrm{dt}, J=14.3,7.0 \mathrm{~Hz}$, $1 \mathrm{H}), 5.64-5.55(\mathrm{~m}, 1 \mathrm{H}), 3.80(\mathrm{~d}, J=7.0 \mathrm{~Hz}, 2 \mathrm{H}), 2.92(\mathrm{td}, J=16.5,6.8 \mathrm{~Hz}, 2 \mathrm{H})$;

${ }^{19} \mathbf{F}\left\{{ }^{1} \mathbf{H}\right\}$ NMR (376 MHz, Chloroform- $d$ ) $\delta-104.90,-110.78$;

${ }^{13} \mathbf{C}\left\{{ }^{1} \mathbf{H}\right\}$ NMR (100 MHz, Chloroform- $\left.d\right) \delta 162.9\left(\mathrm{~d},{ }^{1} J_{C-F}=245.8 \mathrm{~Hz}\right), 161.5\left(\mathrm{t},{ }^{2} J_{C-F}=28.6 \mathrm{~Hz}\right)$, $138.3,137.5\left(\mathrm{~d},{ }^{3} J_{C-F}=10.8 \mathrm{~Hz}\right), 133.9,130.3\left(\mathrm{~d},{ }^{3} J_{C-F}=9.2 \mathrm{~Hz}\right), 129.4\left(\mathrm{t},{ }^{3} J_{C-F}=5.3 \mathrm{~Hz}\right), 129.2$, $128.3,123.8,116.4\left(\mathrm{t},{ }^{1} J_{C-F}=255.3 \mathrm{~Hz}\right), 115.8\left(\mathrm{~d},{ }^{4} J_{C-F}=3.1 \mathrm{~Hz}\right), 112.4\left(\mathrm{~d},{ }^{1} J_{C-F}=21.3 \mathrm{~Hz}\right)$, $108.0\left(\mathrm{~d},{ }^{2} J_{C-F}=26.5 \mathrm{~Hz}\right), 59.8,37.1\left(\mathrm{t},{ }^{2} J_{C-F}=24.4 \mathrm{~Hz}\right)$;

HRMS (ESI) m/z: $\mathrm{C}_{18} \mathrm{H}_{16} \mathrm{~F}_{3} \mathrm{NNaO}_{3} \mathrm{~S}^{+}[\mathrm{M}+\mathrm{Na}]^{+}$Calcd for 406.0695; Found 406.0707.<smiles>O=C(Nc1cccc(C(F)(F)F)c1)C(F)(F)C/C=C/CS(=O)(=O)c1ccccc1</smiles>

4f: $48.1 \mathrm{mg}$, yellow solid, yield: 55\%; purified by flash column chromatography (PE/EA = 4/1); m.p. $103-105^{\circ} \mathrm{C}$;

IR (neat) v 3353, 2950, 1707, 1608, 1557, 1297, 1139, 1106, $696 \mathrm{~cm}^{-1}$;

${ }^{1}$ H NMR (400 MHz, Chloroform- $d$ ) $\delta 8.31$ (s, 1H), 7.95 (s, 1H), 7.86 - 7.75 (m, 3H), 7.66 - 7.59 $(\mathrm{m}, 1 \mathrm{H}), 7.53(\mathrm{t}, J=7.7 \mathrm{~Hz}, 2 \mathrm{H}), 7.50-7.42(\mathrm{~m}, 2 \mathrm{H}), 5.75-5.57(\mathrm{~m}, 2 \mathrm{H}), 3.80(\mathrm{~d}, J=6.9 \mathrm{~Hz}$, $2 \mathrm{H}), 2.93(\mathrm{td}, J=16.4,6.7 \mathrm{~Hz}, 2 \mathrm{H})$;

${ }^{19} \mathbf{F}\left\{{ }^{1} \mathbf{H}\right\}$ NMR (376 MHz, Chloroform- $d$ ) $\delta-62.80,-104.88$;

${ }^{13} \mathbf{C}\left\{{ }^{1} \mathbf{H}\right\}$ NMR $(100 \mathrm{MHz}$, Chloroform- $d) \delta 161.7\left(\mathrm{t},{ }^{2} J_{C-F}=28.9 \mathrm{~Hz}\right), 138.3,136.6,133.9,131.6$ $\left(\mathrm{q},{ }^{2} J_{C-F}=32.7 \mathrm{~Hz}\right), 129.8,129.4\left(\mathrm{t},{ }^{3} J_{C-F}=5.4 \mathrm{~Hz}\right), 129.2,128.3,123.8,123.5,123.7\left(\mathrm{q},{ }^{1} J_{C-F}=\right.$ 
$272.5 \mathrm{~Hz}), 122.2\left(\mathrm{q},{ }^{3} J_{C-F}=3.9 \mathrm{~Hz}\right), 117.3\left(\mathrm{q},{ }^{3} J_{C-F}=4.0 \mathrm{~Hz}\right), 116.4\left(\mathrm{t},{ }^{1} J_{C-F}=255.5 \mathrm{~Hz}\right), 59.8$, $37.2\left(\mathrm{t},{ }^{2} J_{C-F}=24.4 \mathrm{~Hz}\right)$;

HRMS (ESI) m/z: $\mathrm{C}_{19} \mathrm{H}_{16} \mathrm{~F}_{5} \mathrm{NNaO}_{3} \mathrm{~S}^{+}[\mathrm{M}+\mathrm{Na}]^{+}$Calcd for 456.0663; Found 456.0673.<smiles>O=C(Nc1ccc(F)cc1)C(F)(F)C/C=C/CS(=O)(=O)c1ccccc1</smiles>

4g: $56.3 \mathrm{mg}$, white solid, yield: 73\%; purified by flash column chromatography (PE/EA = 4/1);

m.p. $152-154{ }^{\circ} \mathrm{C}$;

IR (neat) v 3364, 3061, 2956, 2920, 2850, 1691, 1529, 1300, 1144, 690, $593 \mathrm{~cm}^{-1}$;

${ }^{1}$ H NMR (400 MHz, DMSO- $\left.d_{6}\right) \delta 10.57$ (s, 1H), $7.87-7.79(\mathrm{~m}, 2 \mathrm{H}), 7.72-7.68(\mathrm{~m}, 3 \mathrm{H}), 7.61(\mathrm{t}$, $J=7.6 \mathrm{~Hz}, 2 \mathrm{H}), 7.21(\mathrm{t}, J=8.9 \mathrm{~Hz}, 2 \mathrm{H}), 5.62(\mathrm{dt}, J=12.9,6.7 \mathrm{~Hz}, 2 \mathrm{H}), 4.14(\mathrm{~d}, J=6.5 \mathrm{~Hz}, 2 \mathrm{H})$, $2.95(\mathrm{td}, J=17.5,6.3 \mathrm{~Hz}, 2 \mathrm{H})$;

${ }^{19} \mathbf{F}\left\{{ }^{1} \mathbf{H}\right\}$ NMR $\left(376 \mathrm{MHz}\right.$, DMSO- $\left.d_{6}\right) \delta-103.72,-117.30 ;$

${ }^{13} \mathbf{C}\left\{{ }^{1} \mathbf{H}\right\}$ NMR $\left(100 \mathrm{MHz}\right.$, DMSO- $\left.d_{6}\right) \delta 161.9\left(\mathrm{t},{ }^{2} J_{C-F}=29.2 \mathrm{~Hz}\right), 159.4\left(\mathrm{~d},{ }^{1} J_{C-F}=241.7 \mathrm{~Hz}\right)$, 138.9, 134.2, $134.0\left(\mathrm{~d},{ }^{4} J_{C-F}=2.7 \mathrm{~Hz}\right), 129.6,129.5\left(\mathrm{t},{ }^{3} J_{C-F}=4.9 \mathrm{~Hz}\right), 128.4,124.0,123.3(\mathrm{~d}$, $\left.{ }^{3} J_{C-F}=8.1 \mathrm{~Hz}\right), 117.1\left(\mathrm{t},{ }^{1} J_{C-F}=253.2 \mathrm{~Hz}\right), 115.9\left(\mathrm{~d},{ }^{2} J_{C-F}=22.4 \mathrm{~Hz}\right), 58.6,37.1\left(\mathrm{t},{ }^{2} J_{C-F}=24.0\right.$ $\mathrm{Hz})$;

HRMS (ESI) m/z: $\mathrm{C}_{18} \mathrm{H}_{16} \mathrm{~F}_{3} \mathrm{NNaO}_{3} \mathrm{~S}^{+}[\mathrm{M}+\mathrm{Na}]^{+}$Calcd for 406.0695; Found 406.0705.<smiles>O=C(Nc1ccc(Br)cc1)C(F)(F)C/C=C/CS(=O)(=O)c1ccccc1</smiles>

4h: $53.4 \mathrm{mg}$, white solid, yield: $60 \%$; purified by flash column chromatography (PE/EA = 4/1);

m.p. $154-156^{\circ} \mathrm{C}$;

IR (neat) v 3357, 2921, 2850, 1691, 1524, 1300, 1145, 729, $594 \mathrm{~cm}^{-1}$;

${ }^{1}$ H NMR $\left(400 \mathrm{MHz}, \mathrm{DMSO}-d_{6}\right) \delta 10.63(\mathrm{~s}, 1 \mathrm{H}), 7.86-7.77$ (m, 2H), $7.74-7.49$ (m, 7H), 5.71 $5.50(\mathrm{~m}, 2 \mathrm{H}), 4.14(\mathrm{~d}, J=6.6 \mathrm{~Hz}, 2 \mathrm{H}), 2.95(\mathrm{td}, J=17.5,6.3 \mathrm{~Hz}, 2 \mathrm{H})$;

${ }^{19} \mathbf{F}\left\{{ }^{1} \mathbf{H}\right\}$ NMR $\left(376 \mathrm{MHz}, \mathrm{DMSO}-d_{6}\right) \delta-103.69$;

${ }^{13} \mathbf{C}\left\{{ }^{1} \mathbf{H}\right\}$ NMR $\left(100 \mathrm{MHz}, \mathrm{DMSO}-d_{6}\right) \delta 162.1\left(\mathrm{t},{ }^{2} J_{C-F}=29.4 \mathrm{~Hz}\right), 138.9,137.1,134.2,132.1$, 129.6, $129.4\left(\mathrm{t},{ }^{3} J_{C-F}=4.8 \mathrm{~Hz}\right), 128.4,124.0,123.2,117.3,117.1\left(\mathrm{t},{ }^{1} J_{C-F}=253.1 \mathrm{~Hz}\right), 58.6,37.1$ $\left(\mathrm{t},{ }^{2} J_{C-F}=24.1 \mathrm{~Hz}\right)$; 
HRMS (ESI) m/z: $\mathrm{C}_{18} \mathrm{H}_{16} \mathrm{BrF}_{2} \mathrm{NNaO}_{3} \mathrm{~S}^{+}[\mathrm{M}+\mathrm{Na}]^{+}$Calcd for 465.9895; Found 465.9903 .<smiles>COc1ccc(NC(=O)C(F)(F)C/C=C/CS(=O)(=O)c2ccccc2)cc1</smiles>

4i: $52.2 \mathrm{mg}$, white solid, yield: $66 \%$; purified by flash column chromatography (PE/EA = 4/1);

m.p. $148-150{ }^{\circ} \mathrm{C}$;

IR (neat) $v 3383,2929,2833,1696,1519,1298,1142,727,597 \mathrm{~cm}^{-1}$;

${ }^{1}$ H NMR $\left(400 \mathrm{MHz}\right.$, DMSO- $\left.d_{6}\right) \delta 10.35(\mathrm{~s}, 1 \mathrm{H}), 7.82(\mathrm{~d}, J=7.6 \mathrm{~Hz}, 2 \mathrm{H}), 7.70(\mathrm{t}, J=7.4 \mathrm{~Hz}, 1 \mathrm{H})$, $7.64-7.50(\mathrm{~m}, 4 \mathrm{H}), 7.00-6.88(\mathrm{~m}, 2 \mathrm{H}), 5.67-5.53(\mathrm{~m}, 2 \mathrm{H}), 4.13(\mathrm{~d}, J=6.5 \mathrm{~Hz}, 2 \mathrm{H}), 3.74(\mathrm{~s}$, $3 \mathrm{H}), 2.93(\mathrm{td}, J=17.5,6.2 \mathrm{~Hz}, 2 \mathrm{H})$;

${ }^{19} \mathbf{F}\left\{{ }^{1} \mathbf{H}\right\}$ NMR $\left(376 \mathrm{MHz}, \mathrm{DMSO}-d_{6}\right) \delta-103.59 ;$

${ }^{13} \mathbf{C}\left\{{ }^{1} \mathbf{H}\right\}$ NMR $\left(100 \mathrm{MHz}, \mathrm{DMSO}-d_{6}\right) \delta 161.6\left(\mathrm{t},{ }^{2} J_{C-F}=29.0 \mathrm{~Hz}\right), 156.8,138.9,134.2,130.6$, 129.6, 128.4, 123.9, 122.9, $117.2\left(\mathrm{t},{ }^{1} J_{C-F}=253.0 \mathrm{~Hz}\right), 114.3,58.6,55.7,37.2\left(\mathrm{t},{ }^{2} J_{C-F}=24.0 \mathrm{~Hz}\right)$;

HRMS (ESI) m/z: $\mathrm{C}_{19} \mathrm{H}_{19} \mathrm{~F}_{2} \mathrm{NNaO}_{4} \mathrm{~S}^{+}[\mathrm{M}+\mathrm{Na}]^{+}$Calcd for 418.0895; Found 418.0902.<smiles>CC(C)c1ccc(NC(=O)C(F)(F)C/C=C/CS(=O)(=O)c2ccccc2)cc1</smiles>

4j: $48.3 \mathrm{mg}$, white solid, yield: 59\%; purified by flash column chromatography $(\mathrm{PE} / \mathrm{EA}=4 / 1)$; m.p. $150-152^{\circ} \mathrm{C}$;

IR (neat) $v 3379,2964,2928,1698,1518,1302,1144,728,596 \mathrm{~cm}^{-1}$;

${ }^{1}$ H NMR (400 MHz, Chloroform- $d$ ) $\delta 7.97(\mathrm{~s}, 1 \mathrm{H}), 7.83(\mathrm{~d}, J=7.6 \mathrm{~Hz}, 2 \mathrm{H}), 7.61(\mathrm{t}, J=7.4 \mathrm{~Hz}$, 1H), $7.53-7.47(\mathrm{~m}, 4 \mathrm{H}), 7.22(\mathrm{~d}, J=8.4 \mathrm{~Hz}, 2 \mathrm{H}), 5.68(\mathrm{dt}, J=14.7,7.2 \mathrm{~Hz}, 1 \mathrm{H}), 5.57(\mathrm{dt}, J=$ 15.1, $6.9 \mathrm{~Hz}, 1 \mathrm{H}), 3.79(\mathrm{~d}, J=7.1 \mathrm{~Hz}, 2 \mathrm{H}), 2.96-72.86(\mathrm{~m}, 3 \mathrm{H}), 1.23(\mathrm{~d}, J=6.9 \mathrm{~Hz}, 6 \mathrm{H})$;

${ }^{19} \mathbf{F}\left\{{ }^{1} \mathbf{H}\right\}$ NMR $(376 \mathrm{MHz}$, Chloroform- $d) \delta-104.89$;

${ }^{13} \mathbf{C}\left\{{ }^{1} \mathbf{H}\right\}$ NMR $(100 \mathrm{MHz}$, Chloroform- $d) \delta 161.2\left(\mathrm{t},{ }^{2} J_{C-F}=28.1 \mathrm{~Hz}\right), 146.6,138.3,133.8,133.6$, $129.5\left(\mathrm{t},{ }^{3} J_{C-F}=5.4 \mathrm{~Hz}\right), 129.2,128.4,127.1,123.6,120.5,116.6\left(\mathrm{t},{ }^{1} J_{C-F}=255.2 \mathrm{~Hz}\right), 59.8,37.2$ $\left(\mathrm{t},{ }^{2} J_{C-F}=24.5 \mathrm{~Hz}\right), 33.7,23.9$;

HRMS (ESI) m/z: $\mathrm{C}_{21} \mathrm{H}_{23} \mathrm{~F}_{2} \mathrm{NNaO}_{3} \mathrm{~S}^{+}[\mathrm{M}+\mathrm{Na}]^{+}$Calcd for 430.1259; Found 430.1263 . 
<smiles>Cc1ccc(NC(=O)C(F)(F)C/C=C/CS(=O)(=O)c2ccccc2)cc1</smiles>

4k: $52.4 \mathrm{mg}$, pale yellow solid, yield: 69\%; purified by flash column chromatography (PE/EA = $4 / 1)$

m.p. $130-132{ }^{\circ} \mathrm{C}$;

IR (neat) $v 3383,2919,1700,1521,1301,1143,726,597 \mathrm{~cm}^{-1}$;

${ }^{1}$ H NMR (400 MHz, Chloroform- $d$ ) $\delta 7.98(\mathrm{~s}, 1 \mathrm{H}), 7.82(\mathrm{~d}, J=7.5 \mathrm{~Hz}, 2 \mathrm{H}), 7.61(\mathrm{t}, J=7.4 \mathrm{~Hz}$, $1 \mathrm{H}), 7.51(\mathrm{t}, J=7.7 \mathrm{~Hz}, 2 \mathrm{H}), 7.45(\mathrm{~d}, J=8.4 \mathrm{~Hz}, 2 \mathrm{H}), 5.67(\mathrm{dt}, J=14.7,7.2 \mathrm{~Hz}, 1 \mathrm{H}), 5.57(\mathrm{dt}, J=$ $15.3,7.0 \mathrm{~Hz}, 1 \mathrm{H}), 3.78(\mathrm{~d}, J=7.1 \mathrm{~Hz}, 2 \mathrm{H}), 2.91(\mathrm{td}, J=16.6,6.9 \mathrm{~Hz}, 2 \mathrm{H}), 2.33(\mathrm{~s}, 3 \mathrm{H})$;

${ }^{19} \mathbf{F}\left\{{ }^{1} \mathbf{H}\right\}$ NMR $(376 \mathrm{MHz}$, Chloroform- $d) \delta-104.90 ;$

${ }^{13} \mathbf{C}\left\{{ }^{1} \mathbf{H}\right\}$ NMR $(100 \mathrm{MHz}$, Chloroform- $d) \delta 161.2\left(\mathrm{t},{ }^{2} J_{C-F}=28.3 \mathrm{~Hz}\right), 138.2,135.5,133.8,133.4$, $129.7,129.6\left(\mathrm{t},{ }^{3} J_{C-F}=5.3 \mathrm{~Hz}\right), 129.2,128.4,123.6,120.4,116.6\left(\mathrm{t},{ }^{1} J_{C-F}=255.2 \mathrm{~Hz}\right), 59.80,37.2$ $\left(\mathrm{t},{ }^{2} J_{C-F}=24.4 \mathrm{~Hz}\right), 20.93$;

HRMS (ESI) m/z: $\mathrm{C}_{19} \mathrm{H}_{19} \mathrm{~F}_{2} \mathrm{NNaO}_{3} \mathrm{~S}^{+}[\mathrm{M}+\mathrm{Na}]^{+}$Calcd for 402.0946; Found 402.0952.<smiles>O=C(NCc1ccccc1)C(F)(F)C/C=C/CS(=O)(=O)c1ccccc1</smiles>

4l: $61.3 \mathrm{mg}$, white solid, yield: $80 \%$; purified by flash column chromatography $(\mathrm{PE} / \mathrm{EA}=3 / 1)$; m.p. $86-88{ }^{\circ} \mathrm{C}$;

IR (neat) v $3348,2999,2919,1671,1544,1291,1140,685,553 \mathrm{~cm}^{-1}$;

${ }^{1}$ H NMR (400 MHz, Chloroform- $d$ ) $\delta 7.90-7.75(\mathrm{~m}, 2 \mathrm{H}), 7.62(\mathrm{t}, J=7.4 \mathrm{~Hz}, 1 \mathrm{H}), 7.53(\mathrm{t}, J=$ $7.6 \mathrm{~Hz}, 2 \mathrm{H}), 7.39-7.19(\mathrm{~m}, 5 \mathrm{H}), 6.77(\mathrm{t}, J=5.6 \mathrm{~Hz}, 1 \mathrm{H}), 5.62(\mathrm{dt}, J=14.9,7.3 \mathrm{~Hz}, 1 \mathrm{H}), 5.56-$ $5.43(\mathrm{~m}, 1 \mathrm{H}), 4.45(\mathrm{~d}, J=5.9 \mathrm{~Hz}, 2 \mathrm{H}), 3.72(\mathrm{~d}, J=7.2 \mathrm{~Hz}, 2 \mathrm{H}), 2.83(\mathrm{td}, J=16.6,7.1 \mathrm{~Hz}, 2 \mathrm{H})$;

${ }^{19} \mathbf{F}\left\{{ }^{1} \mathbf{H}\right\}$ NMR $(376 \mathrm{MHz}$, Chloroform- $d) \delta-105.75$;

${ }^{13} \mathbf{C}\left\{{ }^{1} \mathbf{H}\right\}$ NMR $(100 \mathrm{MHz}$, Chloroform- $d) \delta 163.5\left(\mathrm{t},{ }^{2} J_{C-F}=28.5 \mathrm{~Hz}\right), 138.3,136.9,133.9,129.7$ $\left(\mathrm{t},{ }^{3} J_{C-F}=5.3 \mathrm{~Hz}\right), 129.2,128.9,128.4,127.9,127.9,1234,116.6\left(\mathrm{t},{ }^{1} J_{C-F}=254.0 \mathrm{~Hz}\right), 59.8,43.5$, $37.3\left(\mathrm{t},{ }^{2} J_{C-F}=24.5 \mathrm{~Hz}\right)$;

HRMS (ESI) m/z: $\mathrm{C}_{19} \mathrm{H}_{19} \mathrm{~F}_{2} \mathrm{NNaO}_{3} \mathrm{~S}^{+}[\mathrm{M}+\mathrm{Na}]^{+}$Calcd for 402.0946; Found 402.0954 . 
<smiles>O=C(NCc1ccccc1Cl)C(F)(F)C/C=C/CS(=O)(=O)c1ccccc1</smiles>

4m: $64.2 \mathrm{mg}$, yellow solid, yield: 77\%; purified by flash column chromatography (PE/EA = 3/1); m.p. $94-96{ }^{\circ} \mathrm{C}$;

IR (neat) $v 3412,2977,2928,1693,1544,1301,1143,689,524 \mathrm{~cm}^{-1}$;

${ }^{1}$ H NMR (400 MHz, Chloroform- $d$ ) $\delta 7.87-7.76(\mathrm{~m}, 2 \mathrm{H}), 7.65-7.62(\mathrm{~m}, 1 \mathrm{H}), 7.53(\mathrm{t}, J=7.6$ $\mathrm{Hz}, 2 \mathrm{H}), 7.40-7.31(\mathrm{~m}, 2 \mathrm{H}), 7.29-7.21(\mathrm{~m}, 2 \mathrm{H}), 6.83(\mathrm{t}, J=6.1 \mathrm{~Hz}, 1 \mathrm{H}), 5.62(\mathrm{dt}, J=14.9,7.3$ $\mathrm{Hz}, 1 \mathrm{H}), 5.49(\mathrm{dt}, J=15.3,7.1 \mathrm{~Hz}, 1 \mathrm{H}), 4.55(\mathrm{~d}, J=6.1 \mathrm{~Hz}, 2 \mathrm{H}), 3.72(\mathrm{~d}, J=7.3 \mathrm{~Hz}, 2 \mathrm{H}), 2.82(\mathrm{td}$, $J=16.6,7.1 \mathrm{~Hz}, 2 \mathrm{H})$;

${ }^{19} \mathbf{F}\left\{{ }^{1} \mathbf{H}\right\}$ NMR (376 MHz, Chloroform- $\left.d\right) \delta-105.78$;

${ }^{13} \mathbf{C}\left\{{ }^{1} \mathbf{H}\right\}$ NMR $(100 \mathrm{MHz}$, Chloroform- $d) \delta 163.5\left(\mathrm{t},{ }^{2} J_{C-F}=28.6 \mathrm{~Hz}\right), 138.2,134.2,133.8,133.7$, $130.2,129.7,129.6\left(\mathrm{~d},{ }^{3} J_{C-F}=5.3 \mathrm{~Hz}\right), 129.5,129.2,128.4,127.3,123.4,116.5\left(\mathrm{t},{ }^{1} J_{C-F}=254.0 \mathrm{~Hz}\right)$, $59.7,41.5,37.3\left(\mathrm{t},{ }^{2} J_{C-F}=24.4 \mathrm{~Hz}\right)$;

HRMS (ESI) m/z: $\mathrm{C}_{19} \mathrm{H}_{18} \mathrm{ClF}_{2} \mathrm{NNaO}_{3} \mathrm{~S}^{+}[\mathrm{M}+\mathrm{Na}]^{+}$Calcd for 436.0556; Found 436.0569.<smiles>COc1cccc(CNC(=O)C(F)(F)C/C=C/CS(=O)(=O)c2ccccc2)c1</smiles>

4n: $46.4 \mathrm{mg}$, yellow solid, yield: 56\%; purified by flash column chromatography (PE/EA = 3/1); m.p. $83-85^{\circ} \mathrm{C}$;

IR (neat) v $3354,2927,1690,1541,1303,1141,687,527 \mathrm{~cm}^{-1}$;

${ }^{1}$ H NMR (400 MHz, Chloroform- $d$ ) $\delta 7.92-7.77(\mathrm{~m}, 2 \mathrm{H}), 7.67-7.58(\mathrm{~m}, 1 \mathrm{H}), 7.53(\mathrm{t}, J=7.6$ $\mathrm{Hz}, 2 \mathrm{H}), 7.24(\mathrm{t}, J=7.9 \mathrm{~Hz}, 1 \mathrm{H}), 6.89-6.73(\mathrm{~m}, 4 \mathrm{H}), 5.63(\mathrm{dt}, J=14.8,7.3 \mathrm{~Hz}, 1 \mathrm{H}), 5.50(\mathrm{dt}, J=$ $15.3,7.1 \mathrm{~Hz}, 1 \mathrm{H}), 4.42(\mathrm{~d}, J=5.9 \mathrm{~Hz}, 2 \mathrm{H}), 3.77(\mathrm{~s}, 3 \mathrm{H}), 3.74(\mathrm{~d}, J=7.2 \mathrm{~Hz}, 2 \mathrm{H}), 2.84(\mathrm{td}, J=$ $16.5,7.1 \mathrm{~Hz}, 2 \mathrm{H})$;

${ }^{19} \mathbf{F}\left\{{ }^{1} \mathbf{H}\right\}$ NMR $(376 \mathrm{MHz}$, Chloroform- $d) \delta-105.75$;

${ }^{13} \mathbf{C}\left\{{ }^{1} \mathbf{H}\right\}$ NMR (100 MHz, Chloroform- $\left.d\right) \delta 163.5\left(\mathrm{t},{ }^{2} J_{C-F}=28.5 \mathrm{~Hz}\right), 159.9,138.4,138.3,133.8$, $129.9,129.7\left(\mathrm{t},{ }^{3} J_{C-F}=5.3 \mathrm{~Hz}\right), 129.2,128.4,123.4,120.0,116.6\left(\mathrm{t},{ }^{1} J_{C-F}=254.0 \mathrm{~Hz}\right), 113.7$, 113.0, 59.7, 55.2, 43.4, $37.4\left(\mathrm{t},{ }^{2} J_{C-F}=24.5 \mathrm{~Hz}\right)$;

HRMS (ESI) m/z: $\mathrm{C}_{20} \mathrm{H}_{21} \mathrm{~F}_{2} \mathrm{NNaO}_{4} \mathrm{~S}^{+}[\mathrm{M}+\mathrm{Na}]^{+}$Calcd for 432.1052; Found 432.1059. 


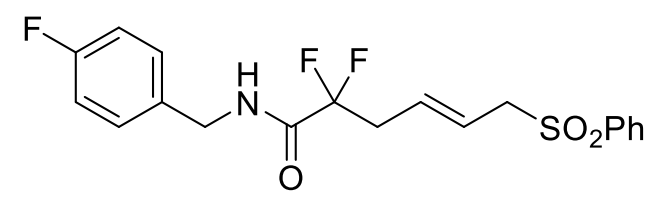

4o: $59.3 \mathrm{mg}$, pale yellow solid, yield: $74 \%$; purified by flash column chromatography $(\mathrm{PE} / \mathrm{EA}=$ $3 / 1)$

m.p. $136-138^{\circ} \mathrm{C}$;

IR (neat) $v 3358,2924,1671,1538,1300,1144,674,536 \mathrm{~cm}^{-1}$;

${ }^{1}$ H NMR $\left(400 \mathrm{MHz}, \mathrm{DMSO}-d_{6}\right) \delta 9.25(\mathrm{t}, J=5.8 \mathrm{~Hz}, 1 \mathrm{H}), 7.91-7.80(\mathrm{~m}, 2 \mathrm{H}), 7.74(\mathrm{t}, J=7.4 \mathrm{~Hz}$, 1H), $7.64(\mathrm{t}, J=7.6 \mathrm{~Hz}, 2 \mathrm{H}), 7.28(\mathrm{dd}, J=8.5,5.6 \mathrm{~Hz}, 2 \mathrm{H}), 7.15(\mathrm{t}, J=8.9 \mathrm{~Hz}, 2 \mathrm{H}), 5.68-5.42$ $(\mathrm{m}, 2 \mathrm{H}), 4.30(\mathrm{~d}, J=6.0 \mathrm{~Hz}, 2 \mathrm{H}), 4.09(\mathrm{~d}, J=6.8 \mathrm{~Hz}, 2 \mathrm{H}), 2.85(\mathrm{td}, J=17.5,6.6 \mathrm{~Hz}, 2 \mathrm{H})$;

${ }^{19} \mathbf{F}\left\{{ }^{1} \mathbf{H}\right\}$ NMR $\left(376 \mathrm{MHz}, \mathrm{DMSO}-d_{6}\right) \delta-104.39,-115.77$;

${ }^{13} \mathbf{C}\left\{{ }^{1} \mathbf{H}\right\}$ NMR $\left(100 \mathrm{MHz}, \mathrm{DMSO}-d_{6}\right) \delta 163.6\left(\mathrm{t},{ }^{2} J_{C-F}=28.9 \mathrm{~Hz}\right), 161.8\left(\mathrm{~d},{ }^{1} J_{C-F}=242.6 \mathrm{~Hz}\right)$, $138.9,135.0\left(\mathrm{~d},{ }^{4} J_{C-F}=3.0 \mathrm{~Hz}\right), 134.2,129.8\left(\mathrm{~d},{ }^{3} J_{C-F}=8.2 \mathrm{~Hz}\right), 129.7,129.6\left(\mathrm{~d},{ }^{3} J_{C-F}=5.6 \mathrm{~Hz}\right)$, $128.4,123.8,117.2\left(\mathrm{t},{ }^{1} J_{C-F}=252.2 \mathrm{~Hz}\right), 115.6\left(\mathrm{~d},{ }^{2} J_{C-F}=21.3 \mathrm{~Hz}\right), 58.6,42.0,37.2\left(\mathrm{t},{ }^{2} J_{C-F}=24.1\right.$ $\mathrm{Hz})$;

HRMS (ESI) m/z: $\mathrm{C}_{19} \mathrm{H}_{18} \mathrm{~F}_{3} \mathrm{NNaO}_{3} \mathrm{~S}^{+}[\mathrm{M}+\mathrm{Na}]^{+}$Calcd for 420.0852; Found 420.0863.<smiles>O=C(NC1CCCC1)C(F)(F)C/C=C/CS(=O)(=O)c1ccccc1</smiles>

4p: $51.4 \mathrm{mg}$, pale yellow solid, yield: $72 \%$; purified by flash column chromatography $(\mathrm{PE} / \mathrm{EA}=$ $3 / 1-2 / 1)$

m.p. $101-103^{\circ} \mathrm{C}$;

IR (neat) $v 3339,2957,1661,1535,1293,1140,1081,739,686,546 \mathrm{~cm}^{-1}$;

${ }^{1}$ H NMR (400 MHz, Chloroform- $\left.d\right) \delta 7.85(\mathrm{~d}, J=7.6 \mathrm{~Hz}, 2 \mathrm{H}), 7.66(\mathrm{t}, J=7.4 \mathrm{~Hz}, 1 \mathrm{H}), 7.56(\mathrm{t}, J$ $=7.7 \mathrm{~Hz}, 2 \mathrm{H}), 6.34(\mathrm{~d}, J=7.6 \mathrm{~Hz}, 1 \mathrm{H}), 5.64(\mathrm{dt}, J=14.9,7.2 \mathrm{~Hz}, 1 \mathrm{H}), 5.53(\mathrm{dt}, J=15.1,7.0 \mathrm{~Hz}$, 1H), $4.18(\mathrm{~h}, J=7.0 \mathrm{~Hz}, 1 \mathrm{H}), 3.78(\mathrm{~d}, J=7.2 \mathrm{~Hz}, 2 \mathrm{H}), 2.82(\mathrm{td}, J=16.8,7.0 \mathrm{~Hz}, 2 \mathrm{H}), 2.04-1.96$ $(\mathrm{m}, 2 \mathrm{H}), 1.76-1.53(\mathrm{~m}, 4 \mathrm{H}), 1.47-1.39(\mathrm{~m}, 2 \mathrm{H})$;

${ }^{19} \mathbf{F}\left\{{ }^{1} \mathbf{H}\right\}$ NMR $\left(376 \mathrm{MHz}\right.$, Chloroform- $d$ ) $\delta-105.82 ;{ }^{13} \mathbf{C}\left\{{ }^{\mathbf{1}} \mathbf{H}\right\}$ NMR $(100 \mathrm{MHz}$, Chloroform- $d$ ) $\delta$ $163.0\left(\mathrm{t},{ }^{2} J_{C-F}=28.1 \mathrm{~Hz}\right), 1383,133.8,129.8\left(\mathrm{t},{ }^{3} J_{C-F}=5.2 \mathrm{~Hz}\right), 129.2,128.4,123.2,116.5\left(\mathrm{t},{ }^{1} J_{C-F}\right.$ $=253.9 \mathrm{~Hz}), 59.8,51.4,37.3\left(\mathrm{t},{ }^{2} J_{C-F}=24.5 \mathrm{~Hz}\right), 32.8,23.7$; 
HRMS (ESI) m/z: $\mathrm{C}_{17} \mathrm{H}_{21} \mathrm{~F}_{2} \mathrm{NNaO}_{3} \mathrm{~S}^{+}[\mathrm{M}+\mathrm{Na}]^{+}$Calcd for 380.1102; Found 380.1108.<smiles>O=C(N1CCCCC1)C(F)(F)C/C=C/CS(=O)(=O)c1ccccc1</smiles>

4q: $42.2 \mathrm{mg}$, yellow oil, yield: 59\%; purified by flash column chromatography (PE/EA = 3/1 $2 / 1)$

IR (neat) $v 2931,2860,1656,1538,1300,1142,686,536 \mathrm{~cm}^{-1}$;

${ }^{1}$ H NMR (400 MHz, Chloroform- $\left.d\right) \delta 7.85(\mathrm{~d}, J=7.4 \mathrm{~Hz}, 2 \mathrm{H}), 7.64(\mathrm{t}, J=7.4 \mathrm{~Hz}, 1 \mathrm{H}), 7.54(\mathrm{t}, J$ $=7.7 \mathrm{~Hz}, 2 \mathrm{H}), 5.72-5.53(\mathrm{~m}, 2 \mathrm{H}), 3.80(\mathrm{~d}, J=6.5 \mathrm{~Hz}, 2 \mathrm{H}), 3.60(\mathrm{t}, J=5.4 \mathrm{~Hz}, 2 \mathrm{H}), 3.53(\mathrm{t}, J=$ $5.5 \mathrm{~Hz}, 2 \mathrm{H}), 2.86(\mathrm{td}, J=17.6,6.1 \mathrm{~Hz}, 2 \mathrm{H}), 1.73-1.51(\mathrm{~m}, 6 \mathrm{H})$;

${ }^{19} \mathbf{F}\left\{{ }^{1} \mathbf{H}\right\}$ NMR (376 MHz, Chloroform- $d$ ) $\delta-98.41$;

${ }^{13} \mathbf{C}\left\{{ }^{1} \mathbf{H}\right\}$ NMR $(100 \mathrm{MHz}$, Chloroform- $d) \delta 161.0\left(\mathrm{t},{ }^{2} J_{C-F}=28.7 \mathrm{~Hz}\right), 138.1,133.7,131.1\left(\mathrm{t},{ }^{3} J_{C-F}\right.$ $=5.3 \mathrm{~Hz}), 129.0,128.5,122.5,118.2\left(\mathrm{t},{ }^{1} J_{C-F}=256.6 \mathrm{~Hz}\right), 60.0,46.7\left(\mathrm{t},{ }^{4} J_{C-F}=6.6 \mathrm{~Hz}\right), 44.4,38.4$ $\left(\mathrm{t},{ }^{2} J_{C-F}=24.4 \mathrm{~Hz}\right), 26.5,25.6,24.4$;

HRMS (ESI) m/z: $\mathrm{C}_{17} \mathrm{H}_{21} \mathrm{~F}_{2} \mathrm{NNaO}_{3} \mathrm{~S}^{+}[\mathrm{M}+\mathrm{Na}]^{+}$Calcd for 380.1102; Found 380.1119.<smiles>O=C(N1CCOCC1)C(F)(F)C/C=C/CS(=O)(=O)c1ccccc1</smiles>

4r: $48.3 \mathrm{mg}$, yellow solid, yield: $67 \%$; purified by flash column chromatography (PE/EA = 3/1 $2 / 1)$

m.p. $113-115^{\circ} \mathrm{C}$;

IR (neat) v 2927, 2859, 1662, 1538, 1300, 1142, 686, $535 \mathrm{~cm}^{-1}$;

${ }^{1}$ H NMR (400 MHz, Chloroform- $d$ ) $\delta 7.90-7.82(\mathrm{~m}, 2 \mathrm{H}), 7.65(\mathrm{t}, J=7.4 \mathrm{~Hz}, 1 \mathrm{H}), 7.54(\mathrm{t}, J=$ $7.6 \mathrm{~Hz}, 2 \mathrm{H}), 5.76-5.49(\mathrm{~m}, 2 \mathrm{H}), 3.80(\mathrm{~d}, J=6.7 \mathrm{~Hz}, 2 \mathrm{H}), 3.74-3.57(\mathrm{~m}, 8 \mathrm{H}), 2.88(\mathrm{td}, J=17.7$, $6.4 \mathrm{~Hz}, 2 \mathrm{H})$;

${ }^{19} \mathbf{F}\left\{{ }^{1} \mathbf{H}\right\}$ NMR $(376 \mathrm{MHz}$, Chloroform- $d$ ) $\delta-98.33$;

${ }^{13} \mathbf{C}\left\{{ }^{1} \mathbf{H}\right\}$ NMR $(100 \mathrm{MHz}$, Chloroform- $d) \delta 161.3\left(\mathrm{t},{ }^{2} J_{C-F}=29.2 \mathrm{~Hz}\right), 138.2,133.7,130.6\left(\mathrm{t},{ }^{3} J_{C-F}\right.$ $=5.3 \mathrm{~Hz}), 129.0,128.5,122.8,118.1(\mathrm{t}, J=256.3 \mathrm{~Hz}), 66.7,66.6,59.9,46.4\left(\mathrm{t},{ }^{4} J_{C-F}=6.2 \mathrm{~Hz}\right)$, $43.3,38.1\left(\mathrm{t},{ }^{2} J_{C-F}=24.1 \mathrm{~Hz}\right)$;

HRMS (ESI) $\mathbf{~ m / z : ~} \mathrm{C}_{16} \mathrm{H}_{19} \mathrm{~F}_{2} \mathrm{NNaO}_{4} \mathrm{~S}^{+}[\mathrm{M}+\mathrm{Na}]^{+}$Calcd for 382.0895; Found 382.0905 . 


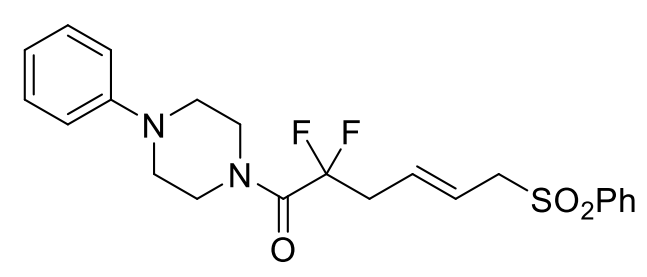

4s: $70.6 \mathrm{mg}$, yellow solid, yield: $81 \%$; purified by flash column chromatography $(\mathrm{PE} / \mathrm{EA}=3 / 1-$ $2 / 1)$

m.p. $105-107^{\circ} \mathrm{C}$;

IR (neat) v 2973, 2827, 1657, 1502, 1290, 1138, 1061, 743, 689, $527 \mathrm{~cm}^{-1}$;

${ }^{1}$ H NMR (400 MHz, Chloroform- $d$ ) $\delta 7.89-7.82(\mathrm{~m}, 2 \mathrm{H}), 7.62(\mathrm{t}, J=7.4 \mathrm{~Hz}, 1 \mathrm{H}), 7.53(\mathrm{t}, J=$ $7.6 \mathrm{~Hz}, 2 \mathrm{H}), 7.33-7.20(\mathrm{~m}, 2 \mathrm{H}), 6.91(\mathrm{~m}, 3 \mathrm{H}), 5.77-5.50(\mathrm{~m}, 2 \mathrm{H}), 3.88-3.69(\mathrm{~m}, 6 \mathrm{H}), 3.27-$ $3.11(\mathrm{~m}, 4 \mathrm{H}), 2.89(\mathrm{td}, J=17.6,6.3 \mathrm{~Hz}, 2 \mathrm{H})$;

${ }^{19} \mathbf{F}\left\{{ }^{1} \mathbf{H}\right\}$ NMR (376 MHz, Chloroform- $d$ ) $\delta-98.02$;

${ }^{13} \mathbf{C}\left\{{ }^{1} \mathbf{H}\right\}$ NMR (100 MHz, Chloroform- $\left.d\right) \delta 161.3\left(\mathrm{t},{ }^{2} J_{C-F}=29.1 \mathrm{~Hz}\right), 150.7,138.2,133.7,130.7$ $\left(\mathrm{t},{ }^{3} J_{C-F}=5.3 \mathrm{~Hz}\right), 129.3,129.1,128.5,122.8,120.8,118.1\left(\mathrm{t},{ }^{1} J_{C-F}=256.3 \mathrm{~Hz}\right), 116.7,59.9,49.9$, $49.3,45.6\left(\mathrm{t},{ }^{4} J_{C-F}=6.1 \mathrm{~Hz}\right), 43.0,38.2\left(\mathrm{t},{ }^{2} J_{C-F}=24.1 \mathrm{~Hz}\right)$;

HRMS (ESI) m/z: $\mathrm{C}_{22} \mathrm{H}_{24} \mathrm{~F}_{2} \mathrm{~N}_{2} \mathrm{NaO}_{3} \mathrm{~S}^{+}[\mathrm{M}+\mathrm{Na}]^{+}$Calcd for 457.1368; Found 457.1377.<smiles>O=C(NCCc1c[nH]c2ccccc12)C(F)(F)C/C=C/CS(=O)(=O)c1ccccc1</smiles>

4t: $69.5 \mathrm{mg}$, yellow solid, yield: $80 \%$; purified by flash column chromatography (PE/EA = 3/1 $2 / 1)$

m.p. $91-93^{\circ} \mathrm{C}$;

IR (neat) v 3396, 3357, 3059, 2944, 1689, 1667, 1555, 1286, 1139, 730, $602 \mathrm{~cm}^{-1}$;

${ }^{1}$ H NMR (400 MHz, Chloroform- $d$ ) $\delta 8.26(\mathrm{~s}, 1 \mathrm{H}), 7.81(\mathrm{~d}, J=7.7 \mathrm{~Hz}, 2 \mathrm{H}), 7.58(\mathrm{~d}, J=7.4 \mathrm{~Hz}$, 2H), $7.51(\mathrm{t}, J=7.6 \mathrm{~Hz}, 2 \mathrm{H}), 7.38(\mathrm{~d}, J=8.1 \mathrm{~Hz}, 1 \mathrm{H}), 7.20(\mathrm{t}, J=7.5 \mathrm{~Hz}, 1 \mathrm{H}), 7.12(\mathrm{t}, J=7.4 \mathrm{~Hz}$, 1H), $7.05(\mathrm{~s}, 1 \mathrm{H}), 6.43(\mathrm{~s}, 1 \mathrm{H}), 5.56(\mathrm{dt}, J=14.9,7.3 \mathrm{~Hz}, 1 \mathrm{H}), 5.40(\mathrm{dt}, J=15.0,7.1 \mathrm{~Hz}, 1 \mathrm{H}), 3.65$ (m, 4H), $3.01(\mathrm{t}, J=6.7 \mathrm{~Hz}, 2 \mathrm{H}), 2.75(\mathrm{td}, J=16.5,7.1 \mathrm{~Hz}, 2 \mathrm{H})$;

${ }^{19} \mathbf{F}\left\{{ }^{1} \mathbf{H}\right\}$ NMR $(376 \mathrm{MHz}$, Chloroform- $d) \delta-105.78 ;$ 
${ }^{13} \mathbf{C}\left\{{ }^{1} \mathbf{H}\right\}$ NMR $\left(100 \mathrm{MHz}\right.$, Chloroform- $d$ ) $\delta 163.5\left(\mathrm{t},{ }^{2} J_{C-F}=28.0 \mathrm{~Hz}\right), 138.3,136.4,133.8,129.8$ $\left(\mathrm{t},{ }^{3} J_{C-F}=5.2 \mathrm{~Hz}\right), 129.2,128.4,127.2,123.1,122.4,122.2,119.6,118.6,116.5\left(\mathrm{t},{ }^{1} J_{C-F}=253.7\right.$ $\mathrm{Hz}), 112.2,111.4,59.7,39.7,37.3\left(\mathrm{t},{ }^{2} J_{C-F}=24.7 \mathrm{~Hz}\right), 24.9$;

HRMS (ESI) m/z: $\mathrm{C}_{22} \mathrm{H}_{22} \mathrm{~F}_{2} \mathrm{~N}_{2} \mathrm{NaO}_{3} \mathrm{~S}^{+}[\mathrm{M}+\mathrm{Na}]^{+}$Calcd for 455.1211; Found 455.1218.<smiles>C/C(=C\CC(F)(F)C(=O)Nc1ccccc1)CS(=O)(=O)Oc1ccccc1</smiles>

5b: $35.1 \mathrm{mg}$, white solid, yield: 46\%; purified by flash column chromatography (PE/EA = 4/1); m.p. $125-127^{\circ} \mathrm{C}$;

IR (neat) v 3359, 3061, 2918, 1693, 1600, 1538, 1446, 1303, 1146, 745, $685 \mathrm{~cm}^{-1}$;

${ }^{1}$ H NMR (400 MHz, Chloroform-d, as a E/Z mixture, 1:0.3) $\delta 8.05$ (s, 1H), 8.00 (s, 0.3H), 7.89 $7.82(\mathrm{~m}, 2.63 \mathrm{H}), 7.60(\mathrm{~m}, 4.17 \mathrm{H}), 7.52(\mathrm{t}, J=7.8 \mathrm{~Hz}, 2.65 \mathrm{H}), 7.37(\mathrm{t}, J=7.9 \mathrm{~Hz}, 2.69 \mathrm{H}), 7.19(\mathrm{t}$, $J=7.4 \mathrm{~Hz}, 1.28 \mathrm{H}), 5.44(\mathrm{t}, J=8.1 \mathrm{~Hz}, 1 \mathrm{H}), 5.24(\mathrm{t}, J=7.6 \mathrm{~Hz}, 0.3 \mathrm{H}), 3.84(\mathrm{~d}, J=8.0 \mathrm{~Hz}, 2 \mathrm{H})$, $3.77(\mathrm{~s}, 0.63 \mathrm{H}), 2.92(\mathrm{t}, J=16.5 \mathrm{~Hz}, 0.72 \mathrm{H}), 2.90(\mathrm{t}, J=17.3 \mathrm{~Hz}, 2 \mathrm{H}), 1.80(\mathrm{~s}, 0.91 \mathrm{H}), 1.54(\mathrm{~s}$, $3 \mathrm{H})$;

${ }^{19} \mathbf{F}\left\{{ }^{1} \mathbf{H}\right\}$ NMR (376 MHz, Chloroform-d) $\delta$-104.03 (2F), -104.66 (0.52F);

${ }^{13} \mathbf{C}\left\{{ }^{1} \mathbf{H}\right\}$ NMR $(100 \mathrm{MHz}$, Chloroform-d, as a $E / Z$ mixture $) \delta 161.57^{\mathrm{M}}\left(\mathrm{t},{ }^{2} J_{C-F}=28.4 \mathrm{~Hz}\right)$, $138.57,{ }^{\mathrm{M}} 138.43,{ }^{\mathrm{m}} 136.51^{\mathrm{M}}\left(\mathrm{t},{ }^{3} J_{C-F}=3.5 \mathrm{~Hz}\right), 136.02,{ }^{\mathrm{m}} 135.96,{ }^{\mathrm{M}} 133.80,{ }^{\mathrm{M}} 133.77,{ }^{\mathrm{m}} 130.54,{ }^{\mathrm{m}}$ $129.22,{ }^{\mathrm{M}} 129.20,{ }^{\mathrm{M}} 129.13,{ }^{\mathrm{m}} 128.38,{ }^{\mathrm{M}} 128.33,{ }^{\mathrm{m}} 125.71,{ }^{\mathrm{M}} 125.64,{ }^{\mathrm{m}} 124.02\left(\mathrm{~d},{ }^{3} J_{C-F}=5.3 \mathrm{~Hz}\right),{ }^{\mathrm{m}}$ $120.40,{ }^{\mathrm{M}} 120.34,{ }^{\mathrm{m}} 119.43^{\mathrm{m}}\left(\mathrm{d},{ }^{1} J_{C-F}=249.7 \mathrm{~Hz}\right), 118.21,{ }^{\mathrm{M}} 116.89^{\mathrm{M}}\left(\mathrm{d},{ }^{1} J_{C-F}=255.9 \mathrm{~Hz}\right), 65.95,{ }^{\mathrm{m}}$ $56.04,{ }^{\mathrm{M}} 43.05^{\mathrm{M}}\left(\mathrm{t},{ }^{2} J_{C-F}=23.4 \mathrm{~Hz}\right), 33.29^{\mathrm{m}}\left(\mathrm{t},{ }^{2} J_{C-F}=24.5 \mathrm{~Hz}\right), 17.61,{ }^{\mathrm{M}} 17.34^{\mathrm{m}}$;

HRMS (ESI) m/z: $\mathrm{C}_{19} \mathrm{H}_{19} \mathrm{~F}_{2} \mathrm{NNaO}_{3} \mathrm{~S}^{+}[\mathrm{M}+\mathrm{Na}]^{+}$Calcd for 402.0946; Found 402.0943.<smiles>C/C(CC(F)(F)C(=O)Nc1ccccc1)=C(/C)CS(=O)(=O)Oc1ccccc1</smiles>

5c: $37.3 \mathrm{mg}$, white solid, yield: $49 \%$; purified by flash column chromatography (PE/EA = 4/1); m.p. $145-147^{\circ} \mathrm{C}$;

IR (neat) $v$ 3362, 2925, 1688, 1601, 1540, 1447, 1304, 1142, 745, $685 \mathrm{~cm}^{-1}$; 
${ }^{1}$ H NMR (400 MHz, Chloroform-d) $\delta 8.10(\mathrm{~s}, 1 \mathrm{H}), 7.89-7.82(\mathrm{~m}, 2 \mathrm{H}), 7.63-7.61$ (m, 3H), 7.51 (t, $J=7.7 \mathrm{~Hz}, 2 \mathrm{H}), 7.38(\mathrm{t}, J=7.9 \mathrm{~Hz}, 2 \mathrm{H}), 7.20(\mathrm{t}, J=7.4 \mathrm{~Hz}, 1 \mathrm{H}), 3.92(\mathrm{~s}, 2 \mathrm{H}), 2.99$ (t, $J=17.5$ $\mathrm{Hz}, 2 \mathrm{H}), 1.86$ (s, 3H), 1.51 (s, 3H);

${ }^{19} \mathbf{F}\left\{{ }^{1} \mathbf{H}\right\}$ NMR (376 MHz, Chloroform-d) $\delta$-103.72;

${ }^{13} \mathbf{C}\left\{{ }^{1} \mathbf{H}\right\}$ NMR (100 MHz, Chloroform-d) $\delta 161.9\left(\mathrm{t},{ }^{2} J_{C-F}=28.5 \mathrm{~Hz}\right), 139.2,136.1,133.8,129.9$ $\left(\mathrm{t},{ }^{3} J_{C-F}=2.8 \mathrm{~Hz}\right), 129.3,129.2,128.3,125.7,124.5,120.4,117.5\left(\mathrm{t},{ }^{1} J_{C-F}=255.9 \mathrm{~Hz}\right), 62.0,38.7$ $\left(\mathrm{d},{ }^{2} J_{C-F}=23.5 \mathrm{~Hz}\right), 20.4,20.2$;

HRMS (ESI) m/z: $\mathrm{C}_{20} \mathrm{H}_{21} \mathrm{~F}_{2} \mathrm{NNaO}_{3} \mathrm{~S}^{+}[\mathrm{M}+\mathrm{Na}]^{+}$Calcd for 416.1102; Found 416.1100 .<smiles>O=C(Nc1ccccc1)C(F)(F)C/C=C/C(CCc1ccccc1)[Sb](=O)(=O)c1ccccc1</smiles>

5d: $47.1 \mathrm{mg}$, white solid, yield: 50\%; purified by flash column chromatography (PE/EA = 4/1); m.p. $126-128^{\circ} \mathrm{C}$;

IR (neat) v 3364, 2987, 1697, 1600, 1538, 1447, 1284, 1140, 735, $686 \mathrm{~cm}^{-1}$;

${ }^{1}$ H NMR (400 MHz, Chloroform-d) $\delta 8.03$ (s, 1H), $7.76(\mathrm{~d}, J=7.4 \mathrm{~Hz}, 2 \mathrm{H}), 7.61-7.57$ (m, 3H), $7.49(\mathrm{t}, J=7.7 \mathrm{~Hz}, 2 \mathrm{H}), 7.34(\mathrm{t}, J=7.9 \mathrm{~Hz}, 2 \mathrm{H}), 7.26-7.13(\mathrm{~m}, 4 \mathrm{H}), 7.02(\mathrm{~d}, J=6.8 \mathrm{~Hz}, 2 \mathrm{H})$, $5.60-5.48(\mathrm{~m}, 2 \mathrm{H}), 3.52-3.46(\mathrm{~m}, 1 \mathrm{H}), 3.12-2.80(\mathrm{~m}, 2 \mathrm{H}), 2.74-2.68(\mathrm{~m}, 1 \mathrm{H}), 2.57-2.31(\mathrm{~m}$ $2 \mathrm{H}), 1.99-1.79(\mathrm{~m}, 1 \mathrm{H})$

${ }^{19} \mathbf{F}\left\{{ }^{1} \mathbf{H}\right\}$ NMR $(376 \mathrm{MHz}$, Chloroform-d) $\delta-104.05(\mathrm{~d}, J=253.5 \mathrm{~Hz}, 1 \mathrm{~F}),-105.48(\mathrm{~d}, J=253.8$ $\mathrm{Hz}, 1 \mathrm{~F})$

${ }^{13} \mathbf{C}\left\{{ }^{1} \mathbf{H}\right\}$ NMR $\left(100 \mathrm{MHz}\right.$, Chloroform-d) $\delta 161.3\left(\mathrm{t},{ }^{2} J_{C-F}=28.3 \mathrm{~Hz}\right), 139.7,137.1,135.9,133.8$, $129.3,129.2,129.1,129.0,128.6\left(\mathrm{t},{ }^{3} J_{C-F}=5.7 \mathrm{~Hz}\right), 128.6,128.4,126.4,125.7,120.3,116.6(\mathrm{t}$, $\left.{ }^{1} J_{C-F}=255.3 \mathrm{~Hz}\right), 68.1,37.3\left(\mathrm{t},{ }^{2} J_{C-F}=24.4 \mathrm{~Hz}\right), 32.3,28.7$;

HRMS (ESI) m/z: $\mathrm{C}_{26} \mathrm{H}_{25} \mathrm{~F}_{2} \mathrm{NNaO}_{3} \mathrm{~S}^{+}[\mathrm{M}+\mathrm{Na}]^{+}$Calcd for 492.1415; Found 492.1407.<smiles>O=C(Nc1ccccc1)C(F)(F)C1C=CC(S(=O)(=O)c2ccccc2)CC1</smiles>

5e: $45.5 \mathrm{mg}$, white solid, yield: 58\%; purified by flash column chromatography (PE/EA = 4/1); m.p. $129-131^{\circ} \mathrm{C}$; 
IR (neat) $v$ 3379, 2987, 1698, 1596, 1533, 1447, 1305, 1144, 725, $687 \mathrm{~cm}^{-1}$;

${ }^{1}$ H NMR (400 MHz, Chloroform-d) $\delta 8.03$ (s, 1H), $7.92-7.80$ (m, 2H), $7.70-7.60$ (m, 1H), 7.60 - 7.51 (m, 4H), 7.37 (t, $J=7.9 \mathrm{~Hz}, 2 \mathrm{H}), 7.20$ (t, $J=7.4 \mathrm{~Hz}, 1 \mathrm{H}), 6.14(\mathrm{dt}, J=10.4,2.0 \mathrm{~Hz}, 1 \mathrm{H})$, $6.04(\mathrm{dt}, J=10.4,2.9 \mathrm{~Hz}, 1 \mathrm{H}), 3.75-3.69(\mathrm{~m}, 1 \mathrm{H}), 3.29-2.96(\mathrm{~m}, 1 \mathrm{H}), 2.32-2.22(\mathrm{~m}, 1 \mathrm{H})$, $1.95-1.87(\mathrm{~m}, 1 \mathrm{H}), 1.82-1.66(\mathrm{~m}, 2 \mathrm{H})$;

${ }^{19} \mathbf{F}\left\{{ }^{1} \mathbf{H}\right\}$ NMR $(376 \mathrm{MHz}$, Chloroform-d) $\delta-110.69(\mathrm{~d}, J=252.7 \mathrm{~Hz}, 1 \mathrm{~F}),-113.22(\mathrm{~d}, J=252.7$ $\mathrm{Hz}, 1 \mathrm{~F})$;

${ }^{13} \mathbf{C}\left\{{ }^{1} \mathbf{H}\right\}$ NMR (100 MHz, Chloroform-d) $\delta 161.18\left(\mathrm{t},{ }^{2} J_{C-F}=28.2 \mathrm{~Hz}\right), 137.25,135.84,133.91$, $129.51\left(\mathrm{t},{ }^{3} J_{C-F}=5.1 \mathrm{~Hz}\right), 129.21,129.19,129.13,125.76,123.13,120.37,117.54\left(\mathrm{t},{ }^{1} J_{C-F}=\right.$ $258.5 \mathrm{~Hz}), 60.23,38.70\left(\mathrm{t},{ }^{2} J_{C-F}=23.2 \mathrm{~Hz}\right), 20.53$, 17.73;

HRMS (ESI) m/z: $\mathrm{C}_{20} \mathrm{H}_{19} \mathrm{~F}_{2} \mathrm{NNaO}_{3} \mathrm{~S}^{+}[\mathrm{M}+\mathrm{Na}]^{+}$Calcd for 414.0946; Found 414.0940.<smiles>O=C(Nc1ccccc1)C(F)(F)C/C=C/C(C1CCCCC1)[R5](=O)(O)c1ccccc1</smiles>

5f: $52.8 \mathrm{mg}$, white solid, yield: 59\%; purified by flash column chromatography (PE/EA = 4/1); m.p. $152-154{ }^{\circ} \mathrm{C}$;

IR (neat) $v$ 3374, 2925, 2854, 1691, 1605, 1551, 1448, 1292, 1136, 1085, 750, $689 \mathrm{~cm}^{-1}$;

${ }^{1}$ H NMR (400 MHz, Chloroform-d) $\delta 8.00$ (s, 1H), $7.84-7.73$ (m, 2H), 7.58 - 7.54 (m, 3H), 7.48 $(\mathrm{t}, J=7.5 \mathrm{~Hz}, 2 \mathrm{H}), 7.35(\mathrm{t}, J=8.0 \mathrm{~Hz}, 2 \mathrm{H}), 7.18(\mathrm{t}, J=7.4 \mathrm{~Hz}, 1 \mathrm{H}), 5.77(\mathrm{dd}, J=15.4,10.5 \mathrm{~Hz}$, $1 \mathrm{H}), 5.24(\mathrm{dt}, \mathrm{J}=15.0,7.3 \mathrm{~Hz}, 1 \mathrm{H}), 3.31(\mathrm{dd}, J=10.4,3.6 \mathrm{~Hz}, 1 \mathrm{H}), 3.07-2.66(\mathrm{~m}, 2 \mathrm{H}), 2.27$ (tq, $\mathrm{J}=11.9,3.4 \mathrm{~Hz}, 1 \mathrm{H}), 2.06-2.03(\mathrm{~m}, 1 \mathrm{H}), 1.72-1.44(\mathrm{~m}, 4 \mathrm{H}), 1.32-1.16(\mathrm{~m}, 2 \mathrm{H}), 1.12-0.91$ $(\mathrm{m}, 3 \mathrm{H})$

${ }^{19} \mathbf{F}\left\{{ }^{1} \mathbf{H}\right\}$ NMR $(376 \mathrm{MHz}$, Chloroform-d) $\delta-103.31(\mathrm{~d}, J=254.1 \mathrm{~Hz}, 1 \mathrm{~F}),-106.45(\mathrm{~d}, J=254.1$ $\mathrm{Hz}, 1 \mathrm{~F})$;

${ }^{13} \mathbf{C}\left\{{ }^{1} \mathbf{H}\right\}$ NMR $(100 \mathrm{MHz}$, Chloroform- $d) \delta 161.3\left(\mathrm{t},{ }^{2} J_{C-F}=28.3 \mathrm{~Hz}\right), 138.4,135.9,133.5,129.2$, 128.8, 128.7, $128.2\left(\mathrm{dd},{ }^{3} J_{C-F}=6.3,4.0 \mathrm{~Hz}\right), 127.9,125.7,120.2,116.7\left(\mathrm{t},{ }^{1} J_{C-F}=255.4 \mathrm{~Hz}\right), 74.4$, $37.2\left(\mathrm{t},{ }^{2} J_{C-F}=24.3 \mathrm{~Hz}\right), 36.7,32.0,28.6,26.3,25.9,25.7$;

HRMS (ESI) m/z: $\mathrm{C}_{24} \mathrm{H}_{27} \mathrm{~F}_{2} \mathrm{NNaO}_{3} \mathrm{~S}^{+}[\mathrm{M}+\mathrm{Na}]^{+}$Calcd for 470.1572; Found 470.1586. 
<smiles>O=C(Nc1ccccc1)C(F)(F)C/C=C/CS(=O)(=O)c1ccc(F)cc1</smiles>

6b: $48.1 \mathrm{mg}$, white solid, yield: 62\%; purified by flash column chromatography (PE/EA = 4/1); m.p. $160-162{ }^{\circ} \mathrm{C}$;

IR (neat) v 3360, 2922, 2850, 1689, 1589, 1143, 1083, 690, $530 \mathrm{~cm}^{-1}$;

${ }^{1}$ H NMR $\left(400 \mathrm{MHz}\right.$, DMSO- $\left.d_{6}\right) \delta 10.50(\mathrm{~s}, 1 \mathrm{H}), 7.95-7.82(\mathrm{~m}, 2 \mathrm{H}), 7.67(\mathrm{~d}, J=8.1 \mathrm{~Hz}, 2 \mathrm{H})$, $7.43(\mathrm{t}, J=8.7 \mathrm{~Hz}, 2 \mathrm{H}), 7.37(\mathrm{t}, J=7.7 \mathrm{~Hz}, 2 \mathrm{H}), 7.16(\mathrm{t}, J=7.2 \mathrm{~Hz}, 1 \mathrm{H}), 5.69-5.54(\mathrm{~m}, 2 \mathrm{H})$, $4.16(\mathrm{~d}, J=6.8 \mathrm{~Hz}, 2 \mathrm{H}), 2.96(\mathrm{td}, J=17.5,6.4 \mathrm{~Hz}, 2 \mathrm{H})$;

${ }^{19} \mathbf{F}\left\{{ }^{1} \mathbf{H}\right\}$ NMR $\left(376 \mathrm{MHz}\right.$, DMSO- $\left.d_{6}\right) \delta-103.63,-104.93$;

${ }^{13} \mathbf{C}\left\{{ }^{1} \mathbf{H}\right\}$ NMR $\left(100 \mathrm{MHz}\right.$, DMSO- $\left.d_{6}\right) \delta 165.5\left(\mathrm{~d},{ }^{1} J_{C-F}=252.5 \mathrm{~Hz}\right), 162.0\left(\mathrm{t},{ }^{2} J_{C-F}=29.3 \mathrm{~Hz}\right)$, 137.6, $135.2\left(\mathrm{~d},{ }^{4} J_{C-F}=2.9 \mathrm{~Hz}\right), 131.7\left(\mathrm{~d},{ }^{3} J_{C-F}=9.9 \mathrm{~Hz}\right), 129.7\left(\mathrm{t},{ }^{3} J_{C-F}=4.8 \mathrm{~Hz}\right), 129.2,125.3$, 124.0, 121.3, $\left.117.1\left(\mathrm{t},{ }^{1} J_{C-F}=253.5 \mathrm{~Hz}\right)\right), 116.8\left(\mathrm{~d},{ }^{2} J_{C-F}=22.8 \mathrm{~Hz}\right), 58.6,37.1\left(\mathrm{t},{ }^{2} J_{C-F}=24.0 \mathrm{~Hz}\right)$; HRMS (ESI) m/z: $\mathrm{C}_{18} \mathrm{H}_{16} \mathrm{~F}_{3} \mathrm{NNaO}_{3} \mathrm{~S}^{+}[\mathrm{M}+\mathrm{Na}]^{+}$Calcd for 406.0695; Found 406.0676.<smiles>O=C(Nc1ccccc1)C(F)(F)C/C=C/CS(=O)(=O)c1ccc(Cl)cc1</smiles>

6c: $50.3 \mathrm{mg}$, white solid, yield: $63 \%$; purified by flash column chromatography (PE/EA = 4/1); m.p. $165-167^{\circ} \mathrm{C}$;

IR (neat) v 3362, 2922, 2850, 1688, 1530, 1146, 1082, 689, $531 \mathrm{~cm}^{-1}$;

${ }^{1}$ H NMR $\left(400 \mathrm{MHz}\right.$, DMSO- $\left.d_{6}\right) \delta 10.50(\mathrm{~s}, 1 \mathrm{H}), 7.82(\mathrm{~d}, J=8.5 \mathrm{~Hz}, 2 \mathrm{H}), 7.72-7.62(\mathrm{~m}, 4 \mathrm{H})$, $7.37(\mathrm{t}, J=7.8 \mathrm{~Hz}, 2 \mathrm{H}), 7.17(\mathrm{t}, J=7.4 \mathrm{~Hz}, 1 \mathrm{H}), 5.69-5.56(\mathrm{~m}, 2 \mathrm{H}), 4.18(\mathrm{~d}, J=6.1 \mathrm{~Hz}, 2 \mathrm{H})$, $2.97(\mathrm{td}, J=17.5,5.8 \mathrm{~Hz}, 2 \mathrm{H})$;

${ }^{19} \mathbf{F}\left\{{ }^{1} \mathbf{H}\right\}$ NMR $\left(376 \mathrm{MHz}, \mathrm{DMSO}-d_{6}\right) \delta-103.60 ;$

${ }^{13} \mathbf{C}\left\{{ }^{1} \mathbf{H}\right\}$ NMR $\left(100 \mathrm{MHz}\right.$, DMSO- $\left.d_{6}\right) \delta 161.9\left(\mathrm{t},{ }^{2} J_{C-F}=29.3 \mathrm{~Hz}\right), 139.3,137.7,137.6,130.4$, $129.9\left(\mathrm{t},{ }^{3} J_{C-F}=5.0 \mathrm{~Hz}\right), 129.8,129.2,125.3,123.8,121.3,117.1\left(\mathrm{t},{ }^{1} J_{C-F}=254.5 \mathrm{~Hz}\right), 58.5,37.1$ $\left(\mathrm{t},{ }^{2} J_{C-F}=24.0 \mathrm{~Hz}\right)$;

HRMS (ESI) m/z: $\mathrm{C}_{18} \mathrm{H}_{16} \mathrm{ClF}_{2} \mathrm{NNaO}_{3} \mathrm{~S}^{+}[\mathrm{M}+\mathrm{Na}]^{+}$Calcd for 422.0400; Found 422.0379 . 
<smiles>O=C(Nc1ccccc1)C(F)(F)C/C=C/CS(=O)(=O)c1ccc(C(F)(F)F)cc1</smiles>

6d: $33.4 \mathrm{mg}$, white solid, yield: 38\%; purified by flash column chromatography (PE/EA = 4/1);

m.p. $180-182^{\circ} \mathrm{C}$;

IR (neat) v 3365, 3058, 2980, 2925, 1687, 1532, 1314, 1121, $690 \mathrm{~cm}^{-1}$;

${ }^{1}$ H NMR $\left(400 \mathrm{MHz}\right.$, DMSO- $\left.d_{6}\right) \delta 10.49(\mathrm{~s}, 1 \mathrm{H}), 8.05(\mathrm{~d}, J=8.2 \mathrm{~Hz}, 2 \mathrm{H}), 7.98(\mathrm{~d}, J=8.1 \mathrm{~Hz}, 2 \mathrm{H})$, $7.67(\mathrm{~d}, J=8.0 \mathrm{~Hz}, 2 \mathrm{H}), 7.36(\mathrm{t}, J=7.8 \mathrm{~Hz}, 2 \mathrm{H}), 7.16(\mathrm{t}, J=7.4 \mathrm{~Hz}, 1 \mathrm{H}), 5.79-5.52(\mathrm{~m}, 2 \mathrm{H})$, $4.26(\mathrm{~d}, J=6.1 \mathrm{~Hz}, 2 \mathrm{H}), 2.97(\mathrm{td}, J=17.5,5.8 \mathrm{~Hz}, 2 \mathrm{H})$;

${ }^{19} \mathbf{F}\left\{{ }^{1} \mathbf{H}\right\}$ NMR $\left(376 \mathrm{MHz}, \mathrm{DMSO}-d_{6}\right) \delta-61.74,-103.71$;

${ }^{13} \mathbf{C}\left\{{ }^{1} \mathbf{H}\right\}$ NMR $\left(100 \mathrm{MHz}\right.$, DMSO- $\left.d_{6}\right) \delta 162.0\left(\mathrm{t},{ }^{2} J_{C-F}=28.3 \mathrm{~Hz}\right), 142.7,137.6,133.8\left(\mathrm{q},{ }^{2} J_{C-F}=\right.$ $32.1 \mathrm{~Hz}), 130.1\left(\mathrm{t},{ }^{3} J_{C-F}=4.8 \mathrm{~Hz}\right), 129.6,129.2,126.8\left(\mathrm{q},{ }^{3} J_{C-F}=3.9 \mathrm{~Hz}\right), 125.3,123.8\left(\mathrm{q},{ }^{1} J_{C-F}=\right.$ $273.1 \mathrm{~Hz}), 123.6,121.3,117.1\left(\mathrm{t},{ }^{1} J_{C-F}=252.9 \mathrm{~Hz}\right), 58.2,37.1\left(\mathrm{t},{ }^{2} J_{C-F}=23.8 \mathrm{~Hz}\right)$;

HRMS (ESI) m/z: $\mathrm{C}_{19} \mathrm{H}_{16} \mathrm{~F}_{5} \mathrm{NNaO}_{3} \mathrm{~S}^{+}[\mathrm{M}+\mathrm{Na}]^{+}$Calcd for 456.0663; Found 456.0641.<smiles>Cc1ccc(S(=O)(=O)C/C=C/CC(F)(F)C(=O)Nc2ccccc2)cc1</smiles>

6e: $55.3 \mathrm{mg}$, pale yellow solid, yield: 73\%; purified by flash column chromatography (PE/EA = $4 / 1)$

m.p. $134-136^{\circ} \mathrm{C}$;

IR (neat) $v$ 3361, 2924, 1689, 1533, 1145, 1084, 689, $530 \mathrm{~cm}^{-1}$;

${ }^{1}$ H NMR $(400 \mathrm{MHz}$, Chloroform- $d$ ) $\delta 8.01(\mathrm{~s}, 1 \mathrm{H}), 7.70(\mathrm{~d}, J=8.3 \mathrm{~Hz}, 2 \mathrm{H}), 7.58(\mathrm{~d}, J=7.7 \mathrm{~Hz}$, 2H), $7.37(\mathrm{t}, J=8.0 \mathrm{~Hz}, 2 \mathrm{H}), 7.30(\mathrm{~d}, J=8.0 \mathrm{~Hz}, 2 \mathrm{H}), 7.20(\mathrm{t}, J=7.4 \mathrm{~Hz}, 1 \mathrm{H}), 5.76-5.48(\mathrm{~m}$, 2H), $3.77(\mathrm{~d}, J=7.0 \mathrm{~Hz}, 2 \mathrm{H}), 2.93(\mathrm{td}, J=16.5,6.7 \mathrm{~Hz}, 2 \mathrm{H}), 2.41(\mathrm{~s}, 3 \mathrm{H})$;

${ }^{19} \mathbf{F}\left\{{ }^{1} \mathbf{H}\right\}$ NMR $(376 \mathrm{MHz}$, Chloroform- $d) \delta-104.89$;

${ }^{13} \mathbf{C}\left\{{ }^{1} \mathbf{H}\right\}$ NMR (100 MHz, Chloroform- $d$ ) $\delta 161.3\left(\mathrm{t},{ }^{2} J_{C-F}=28.4 \mathrm{~Hz}\right), 144.9,136.0,135.4,129.8$, $129.3\left(\mathrm{t},{ }^{3} J_{C-F}=5.4 \mathrm{~Hz}\right), 129.2,128.4,125.7,124.0,120.4,116.6\left(\mathrm{t},{ }^{1} J_{C-F}=255.3 \mathrm{~Hz}\right), 59.9,37.2$ $\left(\mathrm{t},{ }^{2} J_{C-F}=24.5 \mathrm{~Hz}\right), 21.6$;

HRMS (ESI) m/z: $\mathrm{C}_{19} \mathrm{H}_{19} \mathrm{~F}_{2} \mathrm{NNaO}_{3} \mathrm{~S}^{+}[\mathrm{M}+\mathrm{Na}]^{+}$Calcd for 402.0946; Found 402.0930 . 
<smiles>COc1ccc(S(=O)(=O)C/C=C/CC(F)(F)C(=O)Nc2ccccc2)cc1</smiles>

6f: $58.4 \mathrm{mg}$, white solid, yield: $73 \%$; purified by flash column chromatography (PE/EA = 4/1); m.p. $146-148^{\circ} \mathrm{C}$;

IR (neat) $v 3347,2987,2920,1679,1536,1138,1085,690 \mathrm{~cm}^{-1}$;

${ }^{1}$ H NMR $(400 \mathrm{MHz}$, Chloroform- $d$ ) $\delta 7.99(\mathrm{~s}, 1 \mathrm{H}), 7.75(\mathrm{~d}, J=8.9 \mathrm{~Hz}, 2 \mathrm{H}), 7.58(\mathrm{~d}, J=7.8 \mathrm{~Hz}$, 2H), $7.37(\mathrm{t}, J=7.9 \mathrm{~Hz}, 2 \mathrm{H}), 7.20(\mathrm{t}, J=7.4 \mathrm{~Hz}, 1 \mathrm{H}), 6.97(\mathrm{~d}, J=8.9 \mathrm{~Hz}, 2 \mathrm{H}), 5.69$ (dt, $J=14.6$, $7.1 \mathrm{~Hz}, 1 \mathrm{H}), 5.59(\mathrm{dt}, J=15.5,6.9 \mathrm{~Hz}, 1 \mathrm{H}), 3.85(\mathrm{~s}, 3 \mathrm{H}), 3.76(\mathrm{~d}, J=7.1 \mathrm{~Hz}, 2 \mathrm{H}), 2.93(\mathrm{td}, J=$ $16.5,6.9 \mathrm{~Hz}, 2 \mathrm{H})$

${ }^{19} \mathbf{F}\left\{{ }^{1} \mathbf{H}\right\}$ NMR (376 MHz, Chloroform- $\left.d\right) \delta-104.86$;

${ }^{13} \mathbf{C}\left\{{ }^{1} \mathbf{H}\right\}$ NMR (100 MHz, Chloroform- $d$ ) $\delta 163.9,161.3\left(\mathrm{t},{ }^{2} J_{C-F}=28.2 \mathrm{~Hz}\right), 135.9,130.6,129.9$, $129.2,129.18\left(\mathrm{t},{ }^{3} J_{C-F}=5.7 \mathrm{~Hz}\right), 125.7,124.1,120.4,116.6\left(\mathrm{t},{ }^{1} J_{C-F}=256.5 \mathrm{~Hz}\right), 114.3,60.1,55.7$, $37.2\left(\mathrm{t},{ }^{2} J_{C-F}=24.6 \mathrm{~Hz}\right)$;

HRMS (ESI) m/z: $\mathrm{C}_{19} \mathrm{H}_{19} \mathrm{~F}_{2} \mathrm{NNaO}_{4} \mathrm{~S}^{+}[\mathrm{M}+\mathrm{Na}]^{+}$Calcd for 418.0895; Found 418.0876.<smiles>Cc1cc(C)c(S(=O)(=O)C/C=C/CC(F)(F)C(=O)Nc2ccccc2)c(C)c1</smiles>

6g: $46.7 \mathrm{mg}$, white solid, yield: 57\%; purified by flash column chromatography (PE/EA = 4/1); m.p. $122-124^{\circ} \mathrm{C}$;

IR (neat) $v 3348,2938,1691,1534,1308,1132,968,767 \mathrm{~cm}^{-1}$;

${ }^{1}$ H NMR (400 MHz, Chloroform- $d$ ) $\delta 8.09$ (s, 1H), $7.56(\mathrm{~d}, J=8.0 \mathrm{~Hz}, 2 \mathrm{H}), 7.35$ (t, $J=7.9 \mathrm{~Hz}$, 2H), $7.18(\mathrm{t}, J=7.4 \mathrm{~Hz}, 1 \mathrm{H}), 6.93(\mathrm{~s}, 2 \mathrm{H}), 5.71(\mathrm{dt}, J=14.8,7.2 \mathrm{~Hz}, 1 \mathrm{H}), 5.60(\mathrm{dt}, J=15.1,7.0$ Hz, 1H), 3.78 (d, $J=7.2 \mathrm{~Hz}, 2 \mathrm{H}), 2.91(\mathrm{td}, J=16.7,7.0 \mathrm{~Hz}, 2 \mathrm{H}), 2.60$ (s, 6H), 2.27 (s, 3H); ${ }^{19} \mathbf{F}\left\{{ }^{1} \mathbf{H}\right\}$ NMR $(376 \mathrm{MHz}$, Chloroform- $d) \delta-105.01$;

${ }^{13} \mathbf{C}\left\{{ }^{1} \mathbf{H}\right\}$ NMR $(100 \mathrm{MHz}$, Chloroform- $d) \delta 161.4\left(\mathrm{t},{ }^{2} J_{C-F}=28.3 \mathrm{~Hz}\right), 143.6,140.2,135.9,132.2$, $129.23\left(\mathrm{t},{ }^{3} J_{C-F}=5.2 \mathrm{~Hz}\right), 129.2,125.7,123.6,120.4,116.5\left(\mathrm{t},{ }^{1} J_{C-F}=255.3 \mathrm{~Hz}\right), 59.6,37.3(\mathrm{t}$, $\left.{ }^{2} J_{C-F}=24.4 \mathrm{~Hz}\right), 23.0,21.0$;

HRMS (ESI) m/z: $\mathrm{C}_{21} \mathrm{H}_{23} \mathrm{~F}_{2} \mathrm{NNaO}_{3} \mathrm{~S}^{+}[\mathrm{M}+\mathrm{Na}]^{+}$Calcd for 430.1259; Found 430.1269. 
<smiles>O=C(Nc1ccccc1)C(F)(F)C/C=C/CS(=O)(=O)c1ccc2ccccc2c1</smiles>

6h: $48.6 \mathrm{mg}$, white solid, yield: 59\%; purified by flash column chromatography (PE/EA = 4/1); m.p. $142-144^{\circ} \mathrm{C}$;

IR (neat) $v$ 3364, 3052, 2974, 2922, 1690, 1527, 1301, 1126, 732, $690 \mathrm{~cm}^{-1}$;

${ }^{1}$ H NMR (400 MHz, Chloroform- $d$ ) $\delta 8.41(\mathrm{~s}, 1 \mathrm{H}), 8.00-7.86(\mathrm{~m}, 4 \mathrm{H}), 7.80(\mathrm{dd}, J=8.7,1.7 \mathrm{~Hz}$, 1H), $7.70-7.59(\mathrm{~m}, 2 \mathrm{H}), 7.51(\mathrm{~d}, J=7.8 \mathrm{~Hz}, 2 \mathrm{H}), 7.34(\mathrm{t}, J=7.9 \mathrm{~Hz}, 2 \mathrm{H}), 7.18(\mathrm{t}, J=7.4 \mathrm{~Hz}$, 1H), $5.72(\mathrm{dt}, J=14.8,7.3 \mathrm{~Hz}, 1 \mathrm{H}), 5.60(\mathrm{dt}, J=15.2,7.1 \mathrm{~Hz}, 1 \mathrm{H}), 3.86(\mathrm{~d}, J=7.2 \mathrm{~Hz}, 2 \mathrm{H}), 2.91$ (td, $J=16.4,7.1 \mathrm{~Hz}, 2 \mathrm{H})$;

${ }^{19} \mathbf{F}\left\{{ }^{1} \mathbf{H}\right\}$ NMR (376 MHz, Chloroform- $\left.d\right) \delta-104.83$;

${ }^{13} \mathbf{C}\left\{{ }^{1} \mathbf{H}\right\}$ NMR (100 MHz, Chloroform- $\left.d\right) \delta 161.3\left(\mathrm{t},{ }^{2} J_{C-F}=28.3 \mathrm{~Hz}\right), 135.9,135.4,135.3,132.1$, $130.4,129.53\left(\mathrm{t},{ }^{3} J_{C-F}=5.5 \mathrm{~Hz}\right), 129.47,129.43,129.38,129.2,128.0,127.8,125.7,123.8,122.9$, $120.5,116.5\left(\mathrm{t},{ }^{1} J_{C-F}=255.4 \mathrm{~Hz}\right), 59.9,37.2\left(\mathrm{t},{ }^{2} J_{C-F}=24.5 \mathrm{~Hz}\right)$;

HRMS (ESI) m/z: $\mathrm{C}_{22} \mathrm{H}_{19} \mathrm{~F}_{2} \mathrm{NNaO}_{3} \mathrm{~S}^{+}[\mathrm{M}+\mathrm{Na}]^{+}$Calcd for 438.0946; Found 438.0929.<smiles>O=C(Nc1ccccc1)C(F)(F)C/C=C/CS(=O)(=O)c1cccc2ccccc12</smiles>

6i: $35.2 \mathrm{mg}$, white solid, yield: $42 \%$; purified by flash column chromatography $(\mathrm{PE} / \mathrm{EA}=4 / 1)$; m.p. $116-118{ }^{\circ} \mathrm{C}$;

IR (neat) v 3329, 3048, 2919, 1688, 1530, 1302, 1125, $762,694 \mathrm{~cm}^{-1}$;

${ }^{1}$ H NMR $(400 \mathrm{MHz}$, Chloroform- $d) \delta 8.71(\mathrm{~d}, J=8.6 \mathrm{~Hz}, 1 \mathrm{H}), 8.18(\mathrm{~d}, J=7.3 \mathrm{~Hz}, 1 \mathrm{H}), 8.08(\mathrm{~d}, J$ $=8.2 \mathrm{~Hz}, 1 \mathrm{H}), 7.93(\mathrm{~d}, J=8.0 \mathrm{~Hz}, 2 \mathrm{H}), 7.68(\mathrm{t}, J=7.7 \mathrm{~Hz}, 1 \mathrm{H}), 7.60(\mathrm{t}, J=7.5 \mathrm{~Hz}, 1 \mathrm{H}), 7.57-$ $7.50(\mathrm{~m}, 3 \mathrm{H}), 7.35(\mathrm{t}, J=7.9 \mathrm{~Hz}, 2 \mathrm{H}), 7.19(\mathrm{t}, J=7.4 \mathrm{~Hz}, 1 \mathrm{H}), 5.63(\mathrm{dt}, J=15.0,7.4 \mathrm{~Hz}, 1 \mathrm{H})$ $5.48(\mathrm{dt}, J=15.2,7.2 \mathrm{~Hz}, 1 \mathrm{H}), 3.99(\mathrm{~d}, J=7.3 \mathrm{~Hz}, 2 \mathrm{H}), 2.83(\mathrm{td}, J=16.5,7.2 \mathrm{~Hz}, 2 \mathrm{H})$;

${ }^{19} \mathbf{F}\left\{{ }^{1} \mathbf{H}\right\}$ NMR $(376 \mathrm{MHz}$, Chloroform- $d) \delta-104.99 ;$

${ }^{13} \mathbf{C}\left\{{ }^{1} \mathbf{H}\right\}$ NMR (100 MHz, Chloroform- $\left.d\right) \delta 161.3\left(\mathrm{t},{ }^{2} J_{C-F}=28.4 \mathrm{~Hz}\right), 135.9,135.4,134.1,133.3$, $131.3,129.33,129.3\left(\mathrm{t},{ }^{3} J_{C-F}=5.6 \mathrm{~Hz}\right), 129.2,128.9,128.8,127.0,125.7,124.3,124.0,123.6$, 
$120.4,116.4\left(\mathrm{t},{ }^{1} J_{C-F}=255.3 \mathrm{~Hz}\right), 59.3,37.1\left(\mathrm{t},{ }^{2} J_{C-F}=24.5 \mathrm{~Hz}\right)$;

HRMS (ESI) m/z: $\mathrm{C}_{22} \mathrm{H}_{19} \mathrm{~F}_{2} \mathrm{NNaO}_{3} \mathrm{~S}^{+}[\mathrm{M}+\mathrm{Na}]^{+}$Calcd for 438.0946; Found 438.0922.<smiles>O=C(Nc1ccccc1)C(F)(F)C/C=C/CS(=O)(=O)c1cccnc1</smiles>

6j: $31.6 \mathrm{mg}$, pale yellow solid, yield: $43 \%$; purified by flash column chromatography $(\mathrm{PE} / \mathrm{EA}=$ $2 / 1)$

m.p. $128-130^{\circ} \mathrm{C}$;

IR (neat) v 3354, 2920, 2899, 1693, 1545, 1303, 1145, 703, $689 \mathrm{~cm}^{-1}$;

${ }^{1}$ H NMR (400 MHz, Chloroform- $d$ ) $\delta 9.05(\mathrm{~d}, J=2.2 \mathrm{~Hz}, 1 \mathrm{H}), 8.81(\mathrm{~d}, J=4.4 \mathrm{~Hz}, 1 \mathrm{H}), 8.13(\mathrm{~d}$, $J=8.0 \mathrm{~Hz}, 1 \mathrm{H}), 8.04(\mathrm{~s}, 1 \mathrm{H}), 7.58(\mathrm{~d}, J=7.9 \mathrm{~Hz}, 2 \mathrm{H}), 7.46(\mathrm{dd}, J=7.9,4.9 \mathrm{~Hz}, 1 \mathrm{H}), 7.38(\mathrm{t}, J=$ $7.9 \mathrm{~Hz}, 2 \mathrm{H}), 7.21(\mathrm{t}, J=7.4 \mathrm{~Hz}, 1 \mathrm{H}), 5.74(\mathrm{dt}, J=15.0,7.4 \mathrm{~Hz}, 1 \mathrm{H}), 5.59(\mathrm{dt}, J=15.2,7.2 \mathrm{~Hz}$, $1 \mathrm{H}), 3.85(\mathrm{~d}, J=7.3 \mathrm{~Hz}, 2 \mathrm{H}), 2.95(\mathrm{td}, J=16.6,7.1 \mathrm{~Hz}, 2 \mathrm{H})$;

${ }^{19} \mathbf{F}\left\{{ }^{1} \mathbf{H}\right\}$ NMR (376 MHz, Chloroform-d) $\delta-104.52$;

${ }^{13} \mathbf{C}\left\{{ }^{1} \mathbf{H}\right\}$ NMR $(100 \mathrm{MHz}$, Chloroform- $d) \delta 161.1\left(\mathrm{t},{ }^{2} J_{C-F}=28.2 \mathrm{~Hz}\right), 154.2,149.4,136.5,135.9$, 134.6, $130.4\left(\mathrm{t},{ }^{3} J_{C-F}=5.1 \mathrm{~Hz}\right), 129.2,125.8,123.8,123.2,120.3,116.5\left(\mathrm{t},{ }^{1} J_{C-F}=256.5 \mathrm{~Hz}\right), 60.1$, $37.1\left(\mathrm{t},{ }^{2} J_{C-F}=24.5 \mathrm{~Hz}\right)$;

HRMS (ESI) m/z: $\mathrm{C}_{17} \mathrm{H}_{16} \mathrm{~F}_{2} \mathrm{~N}_{2} \mathrm{NaO}_{3} \mathrm{~S}^{+}[\mathrm{M}+\mathrm{Na}]^{+}$Calcd for 389.0742; Found 389.0730.<smiles>CS(=O)(=O)C/C=C/CC(F)(F)C(=O)Nc1ccccc1</smiles>

6k: $34.3 \mathrm{mg}$, yellow solid, yield: 57\%; purified by flash column chromatography (PE/EA = 2/1); m.p. $94-96{ }^{\circ} \mathrm{C}$;

IR (neat) v 3316, 3023, 2929, 1682, 1538, 1292, 1127, 1085, $686 \mathrm{~cm}^{-1}$;

${ }^{1}$ H NMR (400 MHz, Chloroform- $d$ ) $\delta 8.10(\mathrm{~s}, 1 \mathrm{H}), 7.58(\mathrm{~d}, J=8.7 \mathrm{~Hz}, 2 \mathrm{H}), 7.36(\mathrm{t}, J=8.0 \mathrm{~Hz}$, 2H), $7.20(\mathrm{t}, J=7.4 \mathrm{~Hz}, 1 \mathrm{H}), 6.01-5.72(\mathrm{~m}, 2 \mathrm{H}), 3.71(\mathrm{~d}, J=6.2 \mathrm{~Hz}, 2 \mathrm{H}), 3.03(\mathrm{td}, J=16.4,5.9$ $\mathrm{Hz}, 2 \mathrm{H}), 2.79$ (s, 3H);

${ }^{19} \mathbf{F}\left\{{ }^{1} \mathbf{H}\right\}$ NMR $(376 \mathrm{MHz}$, Chloroform- $d) \delta-104.71$;

${ }^{13} \mathbf{C}\left\{{ }^{1} \mathbf{H}\right\}$ NMR $(100 \mathrm{MHz}$, Chloroform- $d) \delta 161.3\left(\mathrm{t},{ }^{2} J_{C-F}=28.3 \mathrm{~Hz}\right), 135.9,129.7\left(\mathrm{t},{ }^{3} J_{C-F}=5.4\right.$ $\mathrm{Hz}), 129.2,125.8,124.1,120.4,116.6\left(\mathrm{t},{ }^{1} J_{C-F}=255.4 \mathrm{~Hz}\right), 58.3,39.2,37.4\left(\mathrm{t},{ }^{2} J_{C-F}=24.4 \mathrm{~Hz}\right)$; 
HRMS (ESI) m/z: $\mathrm{C}_{13} \mathrm{H}_{15} \mathrm{~F}_{2} \mathrm{NNaO}_{3} \mathrm{~S}^{+}[\mathrm{M}+\mathrm{Na}]^{+}$Calcd for 326.0633; Found 326.0625.<smiles>CCS(=O)(=O)C/C=C/CC(F)(F)C(=O)Nc1ccccc1</smiles>

61: $42.4 \mathrm{mg}$, yellow solid, yield: $66 \%$; purified by flash column chromatography $(\mathrm{PE} / \mathrm{EA}=2 / 1)$; m.p. $90-92{ }^{\circ} \mathrm{C}$;

IR (neat) $v 3359,3060,2924,1689,1535,1303,1126,1083,690,531 \mathrm{~cm}^{-1}$;

${ }^{1}$ H NMR (400 MHz, Chloroform- $d$ ) $\delta 8.14(\mathrm{~s}, 1 \mathrm{H}), 7.58(\mathrm{~d}, J=7.8 \mathrm{~Hz}, 2 \mathrm{H}), 7.36(\mathrm{t}, J=7.9 \mathrm{~Hz}$, 2H), $7.19(\mathrm{t}, J=7.4 \mathrm{~Hz}, 1 \mathrm{H}), 6.04-5.57(\mathrm{~m}, 2 \mathrm{H}), 3.68(\mathrm{~d}, J=6.1 \mathrm{~Hz}, 2 \mathrm{H}), 3.01(\mathrm{td}, J=16.5,6.1$ $\mathrm{Hz}, 2 \mathrm{H}), 2.91(\mathrm{q}, J=7.5 \mathrm{~Hz}, 2 \mathrm{H}), 1.29(\mathrm{t}, J=7.5 \mathrm{~Hz}, 3 \mathrm{H})$;

${ }^{19} \mathbf{F}\left\{{ }^{1} \mathbf{H}\right\}$ NMR $(376 \mathrm{MHz}$, Chloroform- $d) \delta-104.75$;

${ }^{13} \mathbf{C}\left\{{ }^{1} \mathbf{H}\right\}$ NMR (100 MHz, Chloroform- $d$ ) $\delta 161.3\left(\mathrm{t},{ }^{2} J_{C-F}=28.3 \mathrm{~Hz}\right), 135.9,129.3\left(\mathrm{t},{ }^{3} J_{C-F}=5.5\right.$ $\mathrm{Hz}), 129.2,125.7,124.0,120.3,116.6\left(\mathrm{t},{ }^{1} J_{C-F}=255.4 \mathrm{~Hz}\right), 55.8,45.8,37.4\left(\mathrm{t},{ }^{2} J_{C-F}=24.5 \mathrm{~Hz}\right)$, 6.3

HRMS (ESI) m/z: $\mathrm{C}_{14} \mathrm{H}_{17} \mathrm{~F}_{2} \mathrm{NNaO}_{3} \mathrm{~S}^{+}[\mathrm{M}+\mathrm{Na}]^{+}$Calcd for 340.0789; Found 340.0766.<smiles>O=C(Nc1ccccc1)C(F)(F)C/C=C/CS(=O)(=O)C1CC1</smiles>

6m: $45.2 \mathrm{mg}$, white solid, yield: 69\%; purified by flash column chromatography (PE/EA = 3/1); m.p. $89-91{ }^{\circ} \mathrm{C}$;

IR (neat) v 3340, 3050, 2927, 1682, 1536, 1290, 1086, 721, $656 \mathrm{~cm}^{-1}$;

${ }^{1}$ H NMR $(400 \mathrm{MHz}$, Chloroform- $d$ ) $\delta 8.13(\mathrm{~s}, 1 \mathrm{H}), 7.58(\mathrm{~d}, J=7.9 \mathrm{~Hz}, 2 \mathrm{H}), 7.36(\mathrm{t}, J=7.9 \mathrm{~Hz}$, 2H), $7.19(\mathrm{t}, J=7.4 \mathrm{~Hz}, 1 \mathrm{H}), 5.93-5.83(\mathrm{~m}, 2 \mathrm{H}), 3.73(\mathrm{~d}, J=5.6 \mathrm{~Hz}, 2 \mathrm{H}), 3.03(\mathrm{td}, J=16.3,4.7$ $\mathrm{Hz}, 2 \mathrm{H}), 2.33$ (tt, $J=8.0,4.8 \mathrm{~Hz}, 1 \mathrm{H}), 1.19-1.15(\mathrm{~m}, 2 \mathrm{H}), 0.97-0.89(\mathrm{~m}, 2 \mathrm{H})$;

${ }^{19} \mathbf{F}\left\{{ }^{1} \mathbf{H}\right\}$ NMR $(376 \mathrm{MHz}$, Chloroform- $d) \delta-104.79$;

${ }^{13} \mathbf{C}\left\{{ }^{1} \mathbf{H}\right\}$ NMR $\left(100 \mathrm{MHz}\right.$, Chloroform-d) $\delta 161.4\left(\mathrm{t},{ }^{2} J_{C-F}=28.3 \mathrm{~Hz}\right), 135.9,129.2,129.2\left(\mathrm{t},{ }^{3} J_{C-F}\right.$ $=5.5 \mathrm{~Hz}), 125.7,124.1,120.3,116.7\left(\mathrm{t},{ }^{1} J_{C-F}=255.3 \mathrm{~Hz}\right), 57.5,37.4\left(\mathrm{t},{ }^{2} J_{C-F}=24.5 \mathrm{~Hz}\right), 28.5$, 4.7 ;

HRMS (ESI) $\mathbf{m} / \mathbf{z}: \mathrm{C}_{15} \mathrm{H}_{17} \mathrm{~F}_{2} \mathrm{NNaO}_{3} \mathrm{~S}^{+}[\mathrm{M}+\mathrm{Na}]^{+}$Calcd for 352.0789; Found 352.0796. 
<smiles>O=C(Nc1ccccc1)C(F)(F)C/C=C/CN1CCOCC1</smiles>

6n: $49.2 \mathrm{mg}$, yellow solid, yield: 79\%; purified by flash column chromatography $\left(\mathrm{PE} / \mathrm{EA} / \mathrm{Et}_{3} \mathrm{~N}=\right.$ 2/1/0.1);

m.p. $80-82{ }^{\circ} \mathrm{C}$;

IR (neat) v 3346, 2920, 2815, 1681, 1537, 1108, 757, $690 \mathrm{~cm}^{-1}$;

${ }^{1}$ H NMR (400 MHz, Chloroform- $d$ ) $\delta 8.02(\mathrm{~s}, 1 \mathrm{H}), 7.55(\mathrm{~d}, J=7.8 \mathrm{~Hz}, 2 \mathrm{H}), 7.36(\mathrm{t}, J=7.9 \mathrm{~Hz}$, 2H), $7.18(\mathrm{t}, J=7.4 \mathrm{~Hz}, 1 \mathrm{H}), 5.76(\mathrm{dt}, J=13.4,6.6 \mathrm{~Hz}, 1 \mathrm{H}), 5.59(\mathrm{dt}, J=15.3,7.1 \mathrm{~Hz}, 1 \mathrm{H}), 3.64$ $-3.54(\mathrm{~m}, 4 \mathrm{H}), 3.01-2.86(\mathrm{~m}, 4 \mathrm{H}), 2.35(\mathrm{t}, J=4.6 \mathrm{~Hz}, 4 \mathrm{H})$;

${ }^{19} \mathbf{F}\left\{{ }^{1} \mathbf{H}\right\}$ NMR $(376 \mathrm{MHz}$, Chloroform- $d) \delta-105.33$;

${ }^{13} \mathbf{C}\left\{{ }^{1} \mathbf{H}\right\}$ NMR $(100 \mathrm{MHz}$, Chloroform- $d) \delta 161.6\left(\mathrm{t},{ }^{3} J_{C-F}=28.4 \mathrm{~Hz}\right), 136.0,134.1,129.2,125.6$, $122.5\left(\mathrm{t},{ }^{3} J_{C-F}=5.5 \mathrm{~Hz}\right), 120.1,117.2\left(\mathrm{t},{ }^{1} J_{C-F}=254.9 \mathrm{~Hz}\right), 66.8,60.8,53.4,37.2\left(\mathrm{t},{ }^{2} J_{C-F}=24.0\right.$ $\mathrm{Hz})$;

HRMS (ESI) m/z: $\mathrm{C}_{16} \mathrm{H}_{20} \mathrm{~F}_{2} \mathrm{~N}_{2} \mathrm{NaO}_{2}{ }^{+}[\mathrm{M}+\mathrm{Na}]^{+}$Calcd for 333.1385; Found 333.1371.<smiles>O=C(Nc1ccccc1)C(F)(F)C/C=C/CN1CCSCC1</smiles>

6o: $46.3 \mathrm{mg}$, white solid, yield: $71 \%$; purified by flash column chromatography $\left(\mathrm{PE} / \mathrm{EA} / \mathrm{Et}_{3} \mathrm{~N}=\right.$ $4 / 1 / 0.1)$

m.p. $76-78^{\circ} \mathrm{C}$;

IR (neat) v 3304, 2930, 2810, 2768, 1681, 1539, 1104, $748,686 \mathrm{~cm}^{-1}$;

${ }^{1}$ H NMR (400 MHz, Chloroform- $d$ ) $\delta 7.95(\mathrm{~s}, 1 \mathrm{H}), 7.56(\mathrm{~d}, J=8.2 \mathrm{~Hz}, 2 \mathrm{H}), 7.37$ (t, $J=7.7 \mathrm{~Hz}$, 2H), $7.19(\mathrm{t}, J=7.4 \mathrm{~Hz}, 1 \mathrm{H}), 5.75(\mathrm{dt}, J=13.4,6.5 \mathrm{~Hz}, 1 \mathrm{H}), 5.58(\mathrm{dt}, J=14.6,7.0 \mathrm{~Hz}, 1 \mathrm{H}), 2.96$ (m, 4H), $2.60(\mathrm{~m}, 8 \mathrm{H})$;

${ }^{19} \mathbf{F}\left\{{ }^{1} \mathbf{H}\right\}$ NMR $(376 \mathrm{MHz}$, Chloroform- $d) \delta-105.29$;

${ }^{13} \mathbf{C}\left\{{ }^{1} \mathbf{H}\right\}$ NMR (100 MHz, Chloroform- $d$ ) $\delta 161.6\left(\mathrm{t},{ }^{2} J_{C-F}=28.4 \mathrm{~Hz}\right), 136.0,134.2,129.3,125.6$, $122.5\left(\mathrm{t},{ }^{3} J_{C-F}=4.9 \mathrm{~Hz}\right), 120.1,117.2\left(\mathrm{t},{ }^{1} J_{C-F}=254.9 \mathrm{~Hz}\right), 61.1,54.7,37.2\left(\mathrm{t},{ }^{2} J_{C-F}=24.1 \mathrm{~Hz}\right)$, 27.8;

HRMS (ESI) m/z: $\mathrm{C}_{16} \mathrm{H}_{20} \mathrm{~F}_{2} \mathrm{~N}_{2} \mathrm{NaOS}^{+}[\mathrm{M}+\mathrm{Na}]^{+}$Calcd for 349.1157; Found 349.1145. 
<smiles>O=C(Nc1ccccc1)C(F)(F)C/C=C/CN1CCCCC1</smiles>

6p: $36.3 \mathrm{mg}$, yellow oil, yield: $58 \%$; purified by flash column chromatography $\left(\mathrm{PE} / \mathrm{EA} / \mathrm{Et}_{3} \mathrm{~N}=\right.$ $4 / 1 / 0.1)$

IR (neat) $v 3346,2934,2825,2807,1686,1537,1102,755,690 \mathrm{~cm}^{-1}$;

${ }^{1}$ H NMR (400 MHz, Chloroform- $d$ ) $\delta 7.97(\mathrm{~s}, 1 \mathrm{H}), 7.56(\mathrm{~d}, J=7.7 \mathrm{~Hz}, 2 \mathrm{H}), 7.36(\mathrm{t}, J=8.0 \mathrm{~Hz}$, 2H), $7.18(\mathrm{t}, J=7.4 \mathrm{~Hz}, 1 \mathrm{H}), 5.80(\mathrm{dt}, J=14.0,6.7 \mathrm{~Hz}, 1 \mathrm{H}), 5.55(\mathrm{dt}, J=15.1,7.2 \mathrm{~Hz}, 1 \mathrm{H}), 3.01$ $-2.84(\mathrm{~m}, 4 \mathrm{H}), 2.31(\mathrm{t}, J=5.4 \mathrm{~Hz}, 4 \mathrm{H}), 1.50(\mathrm{p}, J=5.6 \mathrm{~Hz}, 4 \mathrm{H}), 1.37$ (p, $J=6.1 \mathrm{~Hz}, 2 \mathrm{H})$;

${ }^{19} \mathbf{F}\left\{{ }^{1} \mathbf{H}\right\}$ NMR $(376 \mathrm{MHz}$, Chloroform- $d) \delta-105.37$;

${ }^{13} \mathbf{C}\left\{{ }^{1} \mathbf{H}\right\}$ NMR (100 MHz, Chloroform- $d$ ) $\delta 161.7\left(\mathrm{t},{ }^{2} J_{C-F}=28.4 \mathrm{~Hz}\right), 136.0,135.0,129.2,125.6$, $121.7\left(\mathrm{t},{ }^{3} J_{C-F}=5.4 \mathrm{~Hz}\right), 120.2,117.3\left(\mathrm{t},{ }^{1} J_{C-F}=254.9 \mathrm{~Hz}\right), 61.2,54.3,37.2\left(\mathrm{t},{ }^{2} J_{C-F}=24.0 \mathrm{~Hz}\right)$, $25.8,24.2$

HRMS (ESI) m/z: $\mathrm{C}_{17} \mathrm{H}_{22} \mathrm{~F}_{2} \mathrm{~N}_{2} \mathrm{NaO}^{+}[\mathrm{M}+\mathrm{Na}]^{+}$Calcd for 331.1592; Found 331.1577.<smiles>CC(=O)N1CCN(C/C=C/CC(F)(F)C(=O)Nc2ccccc2)CC1</smiles>

6q: $42.6 \mathrm{mg}$, pale yellow oil, yield: $61 \%$; purified by flash column chromatography (PE/EA/Et ${ }_{3} \mathrm{~N}$ $=1 / 1 / 0.1)$

IR (neat) v 3304, 2930, 2810, 2768, 1682, 1540, 1103, 748, $686 \mathrm{~cm}^{-1}$;

${ }^{1}$ H NMR (400 MHz, Chloroform- $d$ ) $\delta 8.14$ (s, 1H), 7.59 (d, $\left.J=7.8 \mathrm{~Hz}, 2 \mathrm{H}\right), 7.37$ (t, $J=7.9 \mathrm{~Hz}$, 2H), $7.19(\mathrm{t}, J=7.4 \mathrm{~Hz}, 1 \mathrm{H}), 5.76(\mathrm{dt}, J=15.3,6.6 \mathrm{~Hz}, 1 \mathrm{H}), 5.60(\mathrm{dt}, J=14.9,7.1 \mathrm{~Hz}, 1 \mathrm{H}), 3.53$ $(\mathrm{t}, J=5.1 \mathrm{~Hz}, 2 \mathrm{H}), 3.30(\mathrm{t}, J=5.0 \mathrm{~Hz}, 2 \mathrm{H}), 3.05-2.85(\mathrm{~m}, 4 \mathrm{H}), 2.35-2.32(\mathrm{~m}, 4 \mathrm{H}), 2.02(\mathrm{~s}$, $3 \mathrm{H})$;

${ }^{19} \mathbf{F}\left\{{ }^{1} \mathbf{H}\right\}$ NMR $(376 \mathrm{MHz}$, Chloroform- $d) \delta-105.25 ;$

${ }^{13} \mathbf{C}\left\{{ }^{1} \mathbf{H}\right\}$ NMR $(100 \mathrm{MHz}$, Chloroform- $d) \delta 168.9,161.6\left(\mathrm{t},{ }^{2} J_{C-F}=28.4 \mathrm{~Hz}\right), 136.1,133.8,129.2$, $125.6,122.9\left(\mathrm{t},{ }^{3} J_{C-F}=5.2 \mathrm{~Hz}\right), 120.1,117.2\left(\mathrm{t},{ }^{1} J_{C-F}=255.0 \mathrm{~Hz}\right), 60.2,52.8,52.6,46.0,41.2,37.2$ $\left(\mathrm{t},{ }^{2} J_{C-F}=24.2 \mathrm{~Hz}\right), 21.3$;

HRMS (ESI) m/z: $\mathrm{C}_{18} \mathrm{H}_{23} \mathrm{~F}_{2} \mathrm{~N}_{3} \mathrm{NaO}_{2}^{+}[\mathrm{M}+\mathrm{Na}]^{+}$Calcd for 374.1651; Found 374.1644. 


\section{NMR spectra}

${ }^{1} \mathrm{H}$ NMR (400 MHz, CDCl $),{ }^{19} \mathrm{~F}\left\{{ }^{1} \mathrm{H}\right\}$ NMR $\left(375 \mathrm{MHz}, \mathrm{CDCl}_{3}\right),{ }^{13} \mathrm{C}\left\{{ }^{1} \mathrm{H}\right\}$ NMR (100 MHz, $\mathrm{CDCl}_{3}$ ) spectrum of product $4 \mathrm{a}$

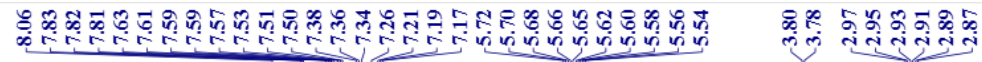

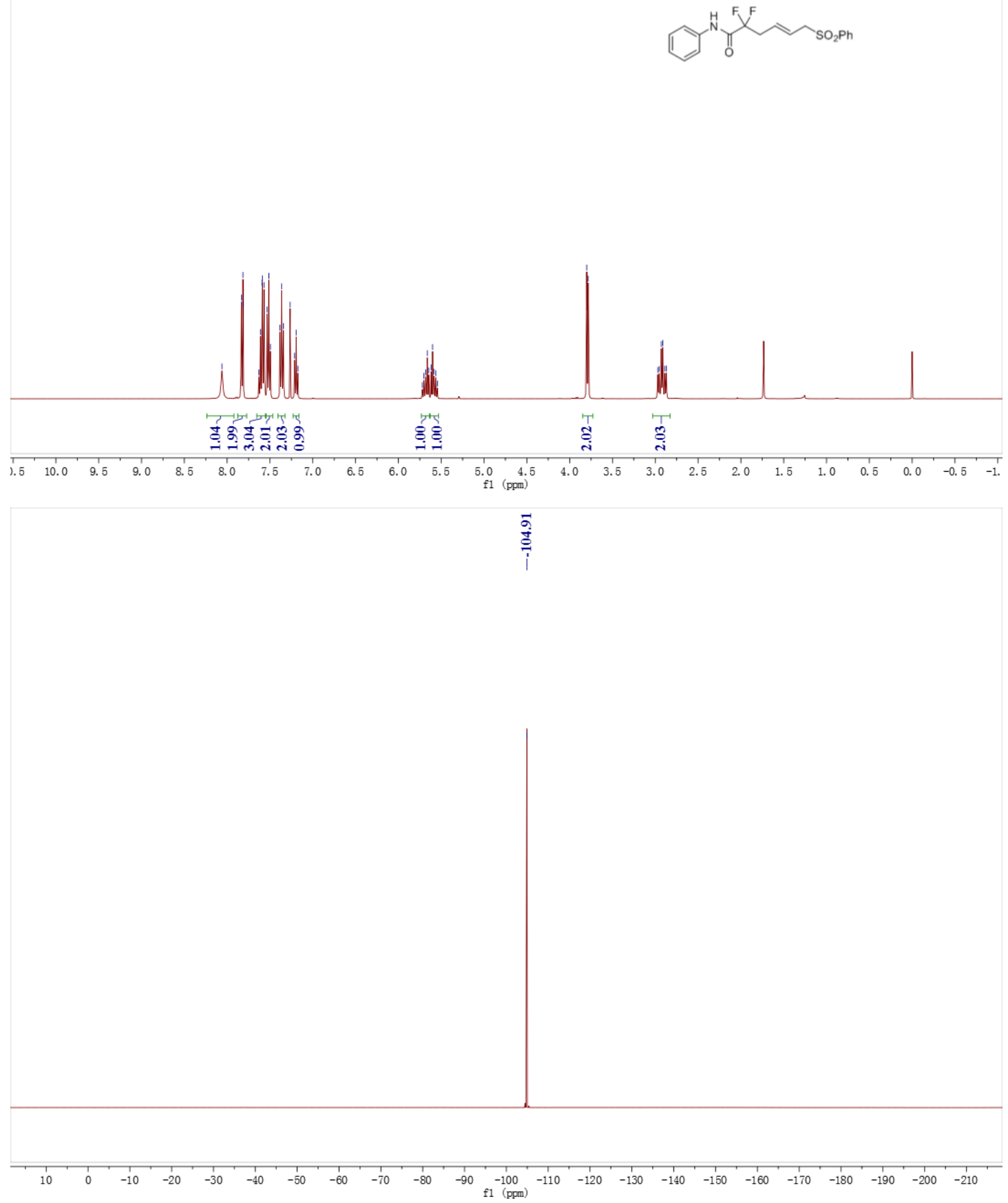




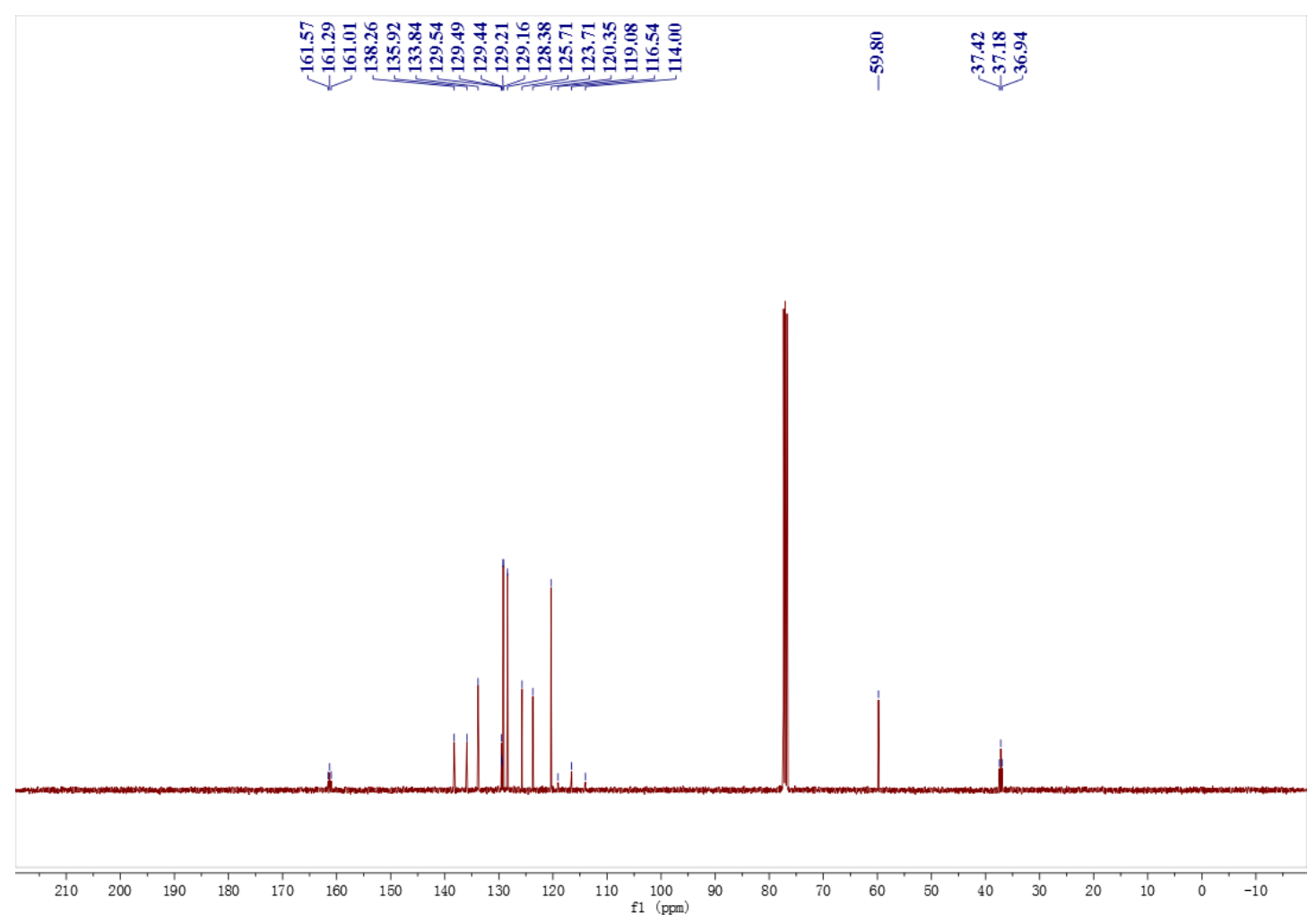


${ }^{1} \mathrm{H}$ NMR (400 MHz, CDCl $),{ }^{19} \mathrm{~F}\left\{{ }^{1} \mathrm{H}\right\}$ NMR $\left(375 \mathrm{MHz}, \mathrm{CDCl}_{3}\right),{ }^{13} \mathrm{C}\left\{{ }^{1} \mathrm{H}\right\}$ NMR (100 MHz, $\mathrm{CDCl}_{3}$ ) spectrum of product $4 \mathrm{~b}$

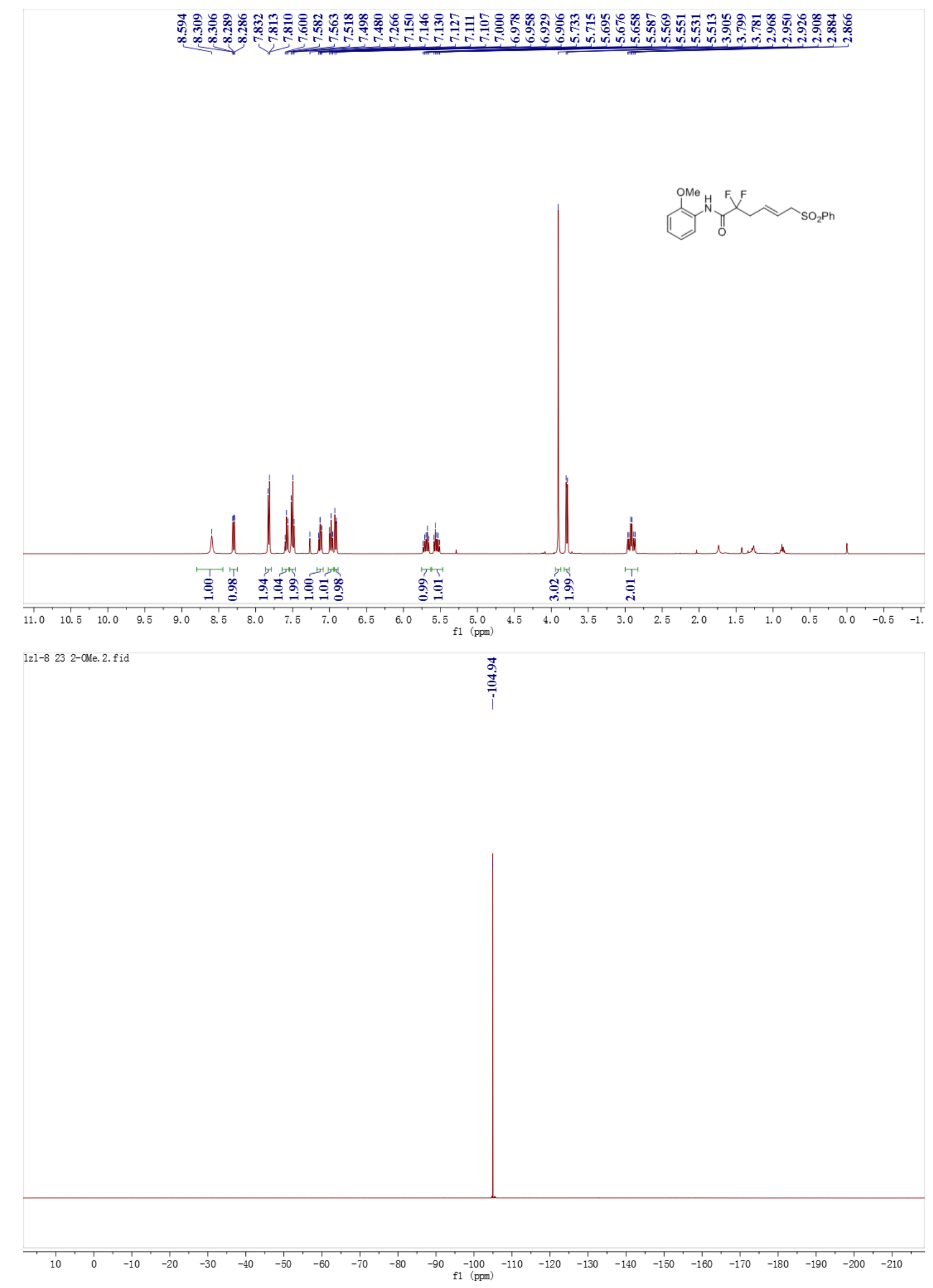




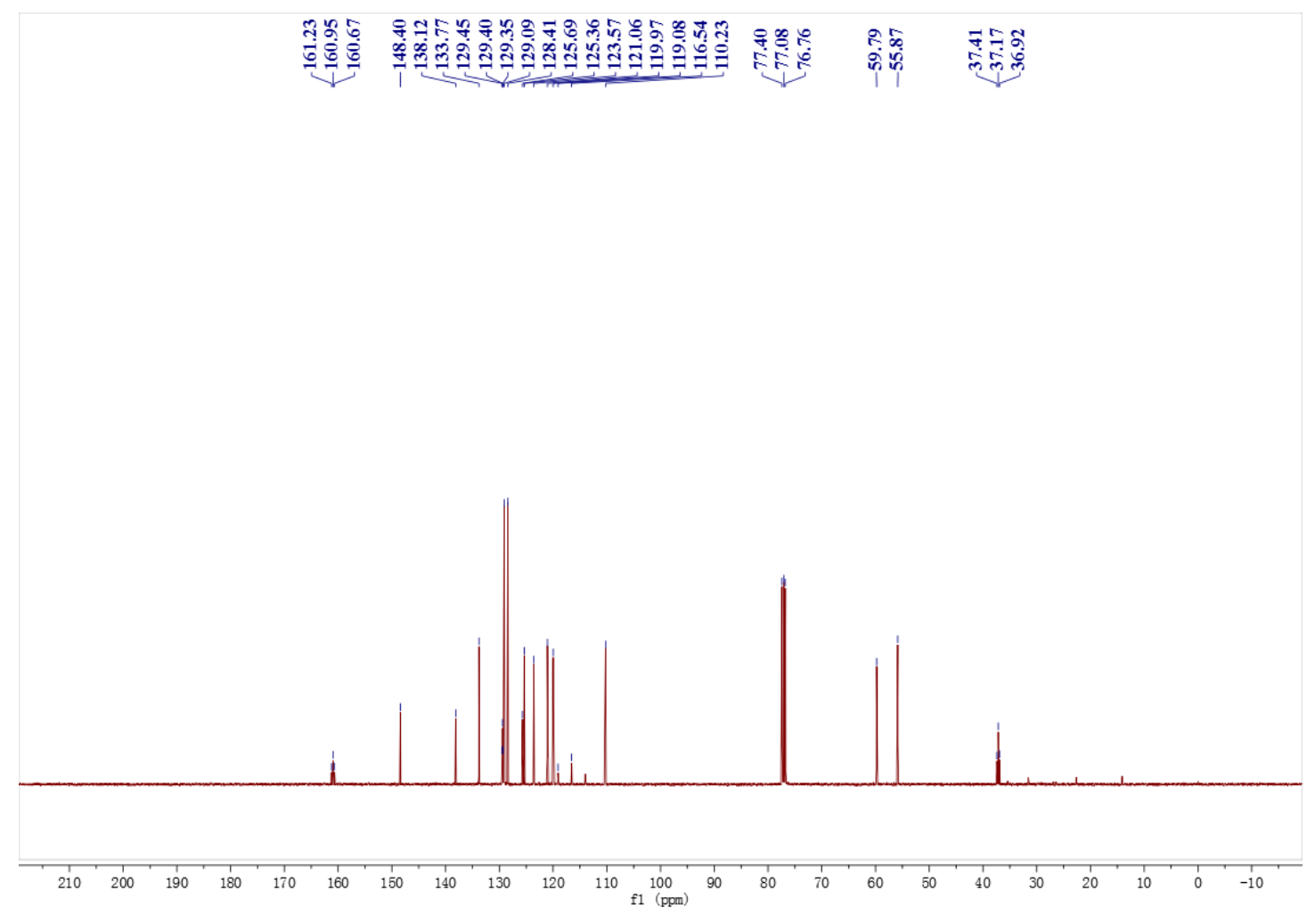


${ }^{1} \mathrm{H}$ NMR (400 MHz, CDCl $),{ }^{19} \mathrm{~F}\left\{{ }^{1} \mathrm{H}\right\}$ NMR $\left(375 \mathrm{MHz}, \mathrm{CDCl}_{3}\right),{ }^{13} \mathrm{C}\left\{{ }^{1} \mathrm{H}\right\}$ NMR (100 MHz, $\mathrm{CDCl}_{3}$ ) spectrum of product $4 \mathrm{c}$

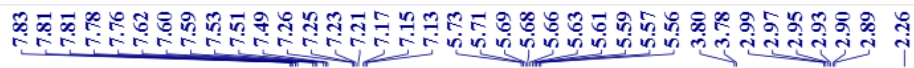
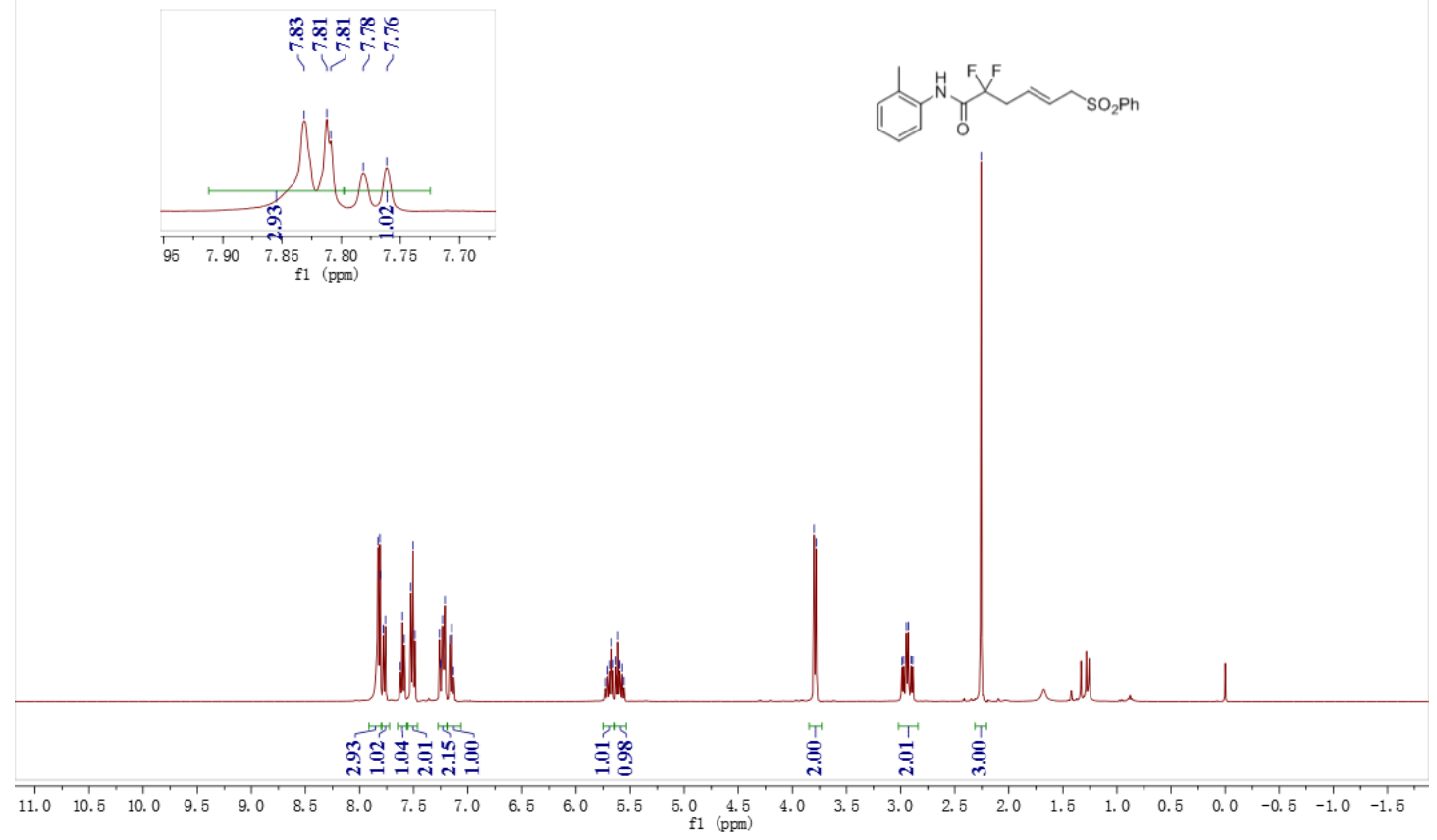

点

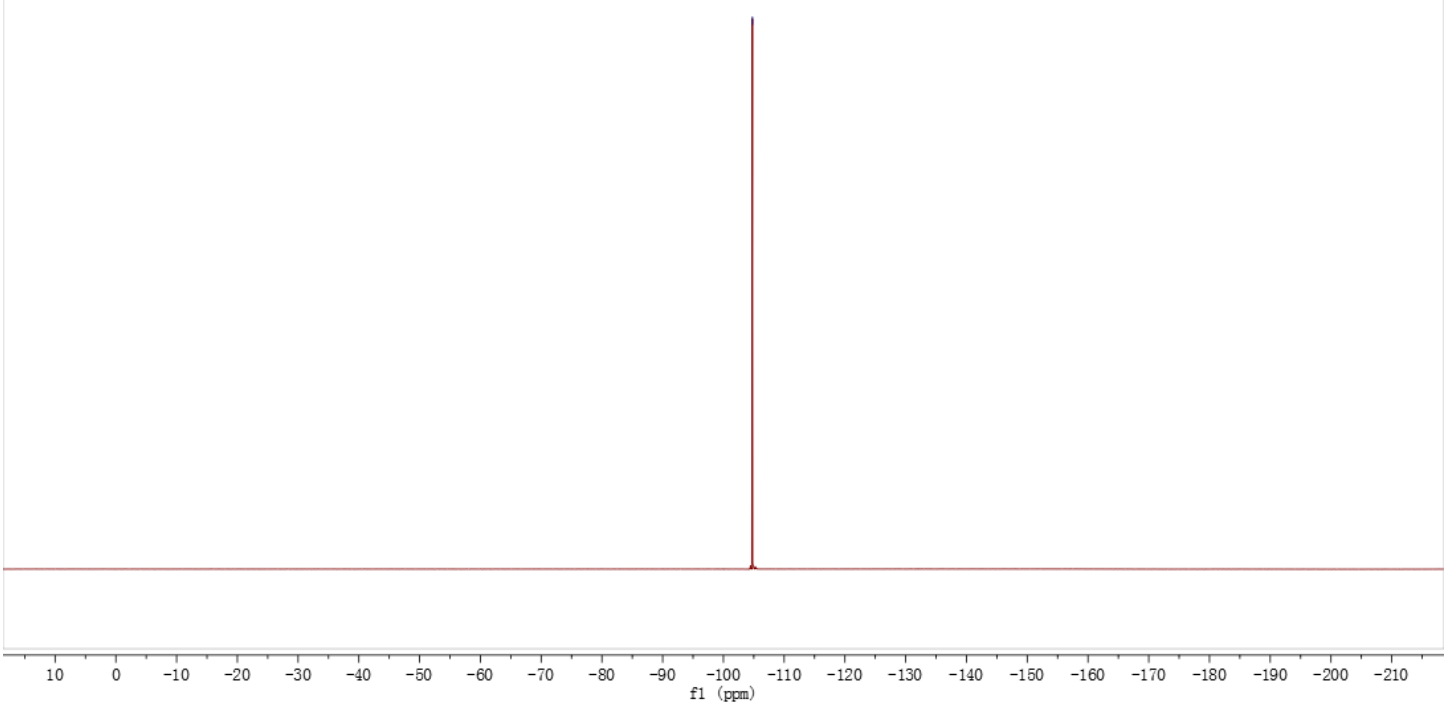




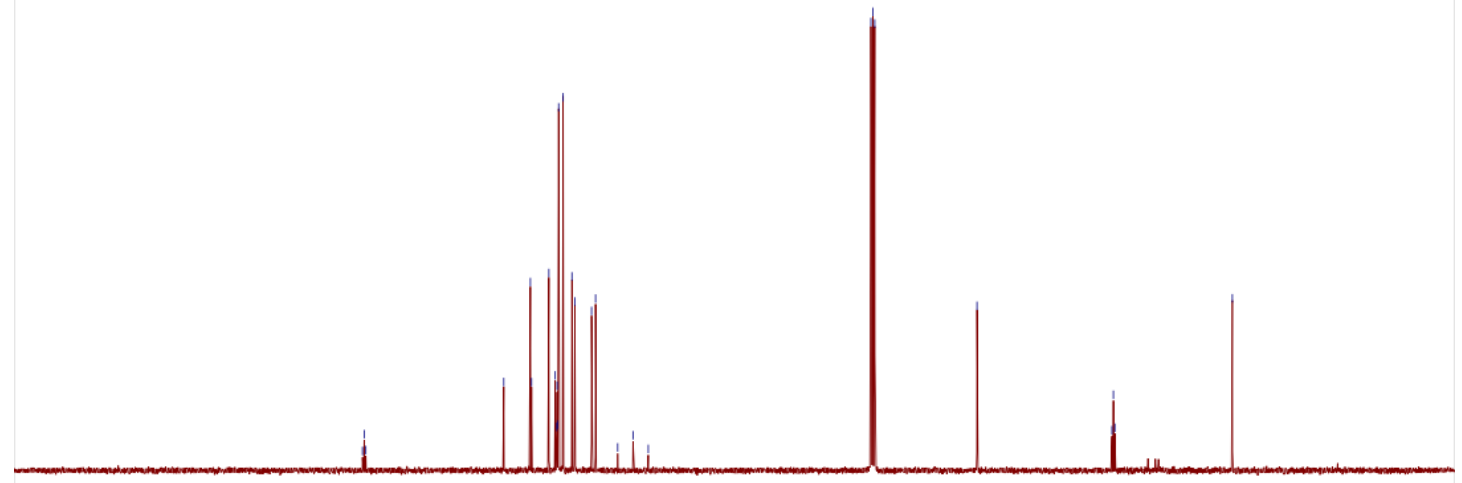

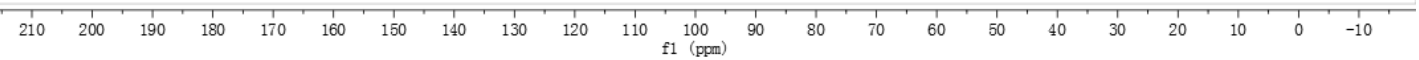


${ }^{1} \mathrm{H}$ NMR (400 MHz, CDCl $),{ }^{19} \mathrm{~F}\left\{{ }^{1} \mathrm{H}\right\}$ NMR $\left(375 \mathrm{MHz}, \mathrm{CDCl}_{3}\right),{ }^{13} \mathrm{C}\left\{{ }^{1} \mathrm{H}\right\}$ NMR (100 MHz, $\mathrm{CDCl}_{3}$ ) spectrum of product $4 \mathrm{~d}$

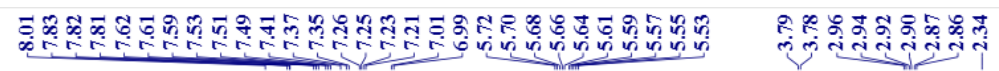
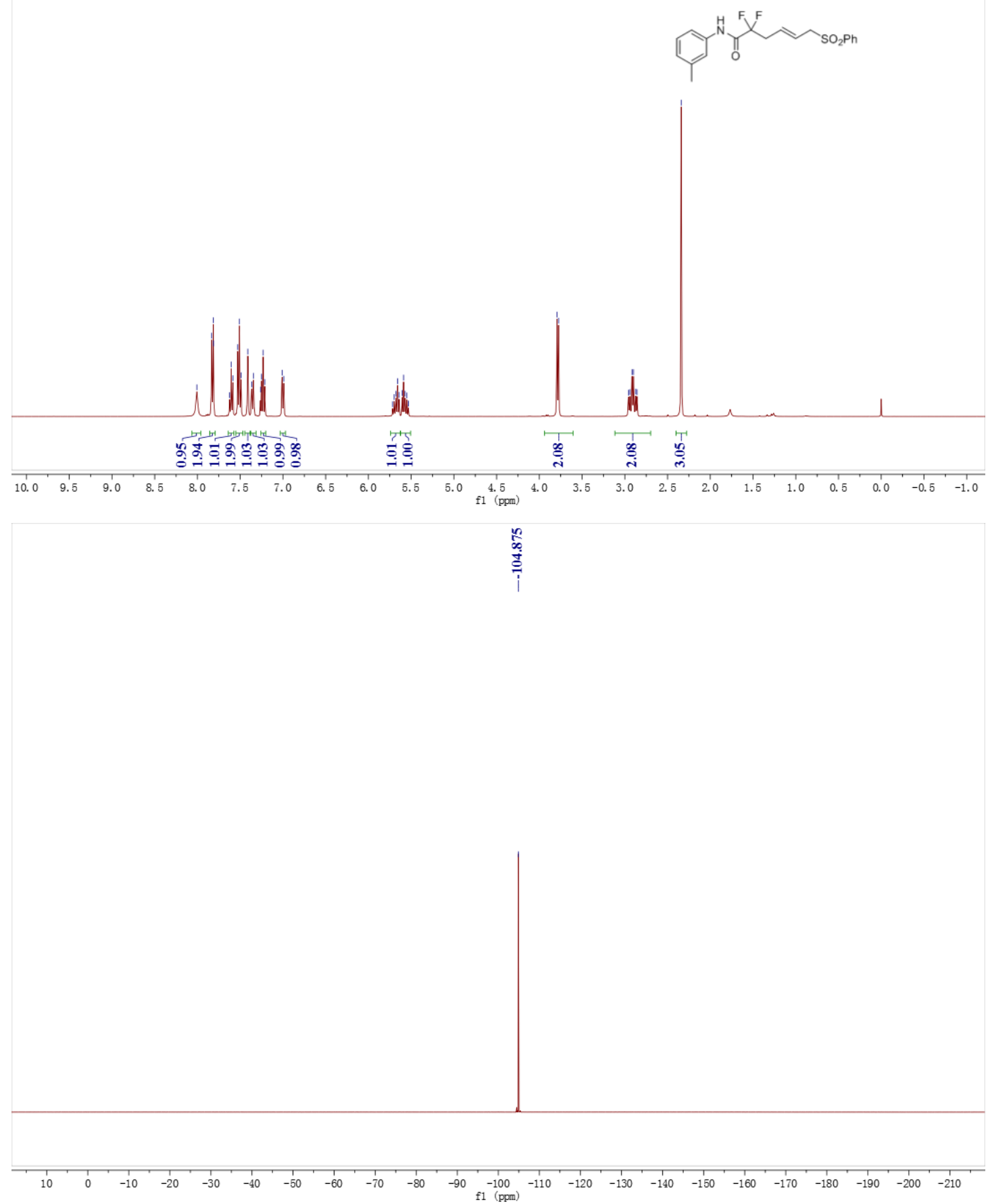


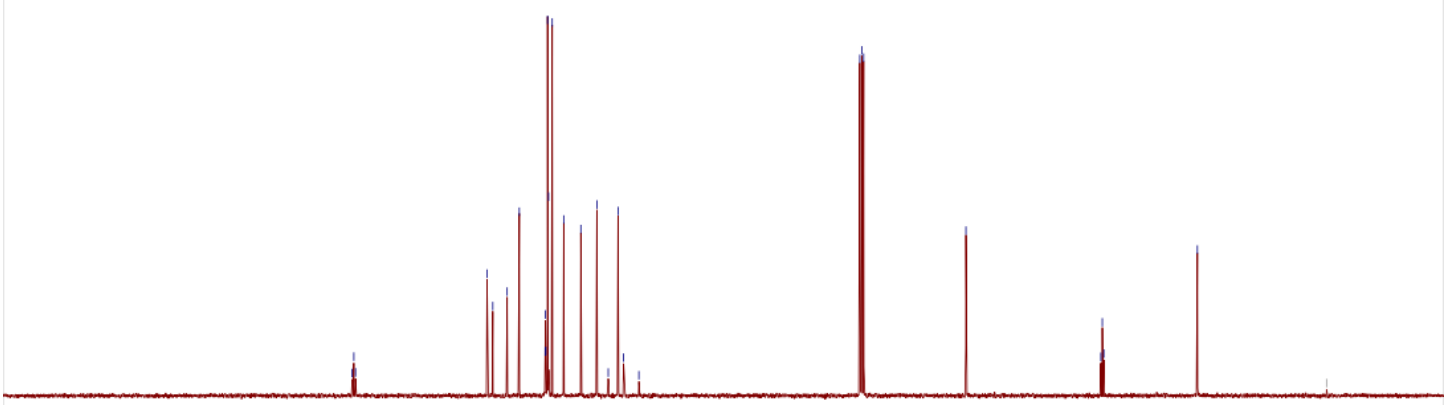

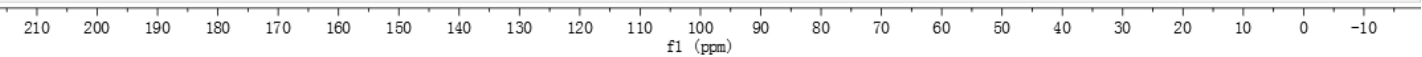


${ }^{1} \mathrm{H}$ NMR (400 MHz, CDCl $),{ }^{19} \mathrm{~F}\left\{{ }^{1} \mathrm{H}\right\}$ NMR $\left(375 \mathrm{MHz}, \mathrm{CDCl}_{3}\right),{ }^{13} \mathrm{C}\left\{{ }^{1} \mathrm{H}\right\}$ NMR (100 MHz, $\mathrm{CDCl}_{3}$ ) spectrum of product $4 \mathrm{e}$

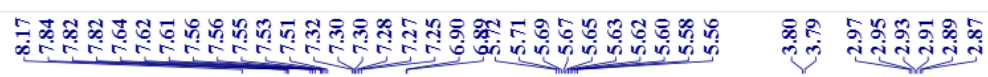

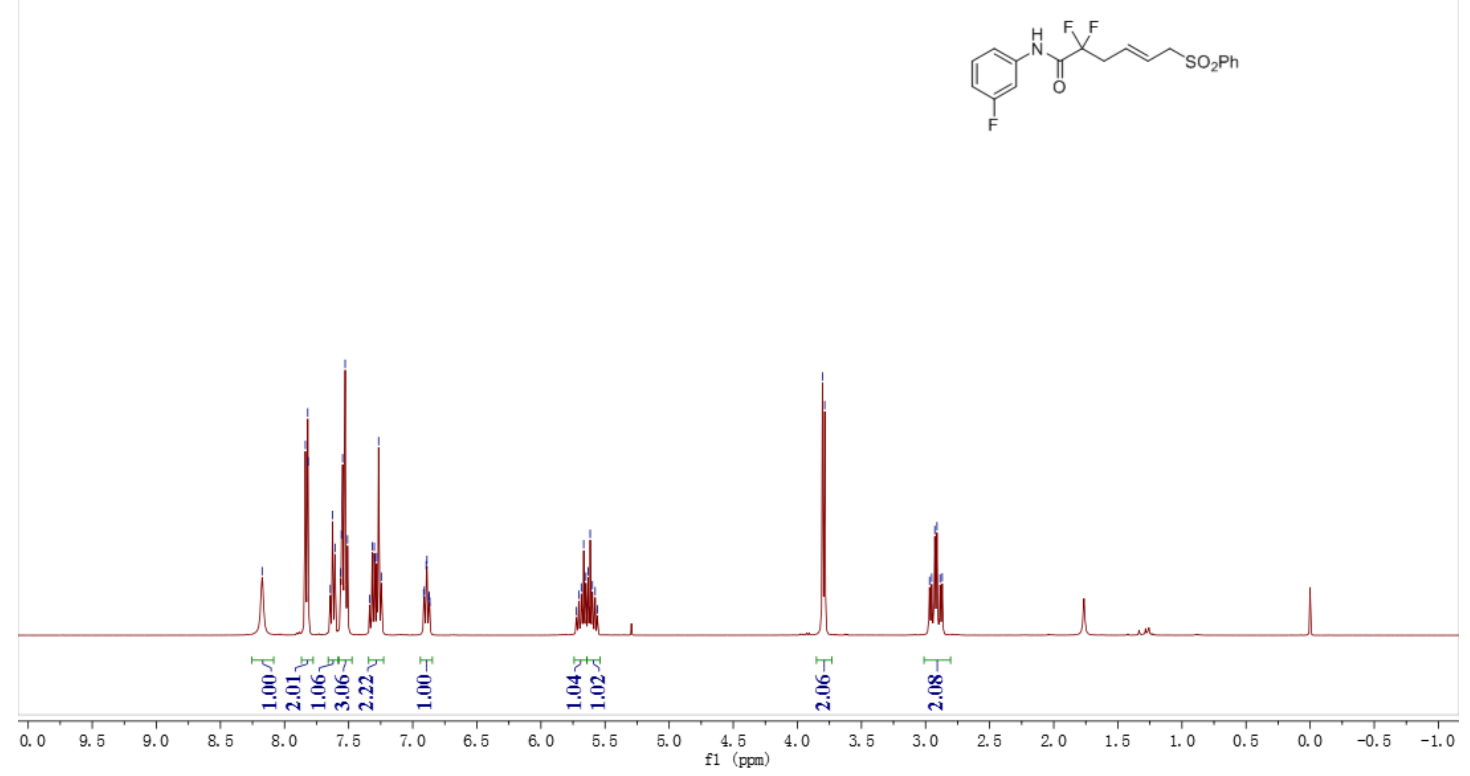

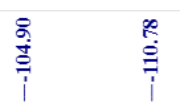

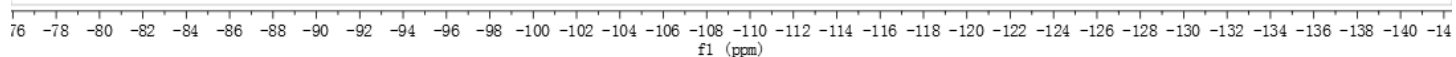




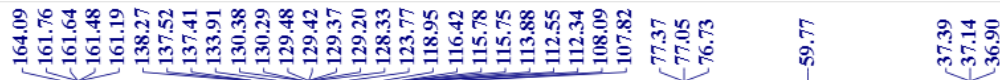

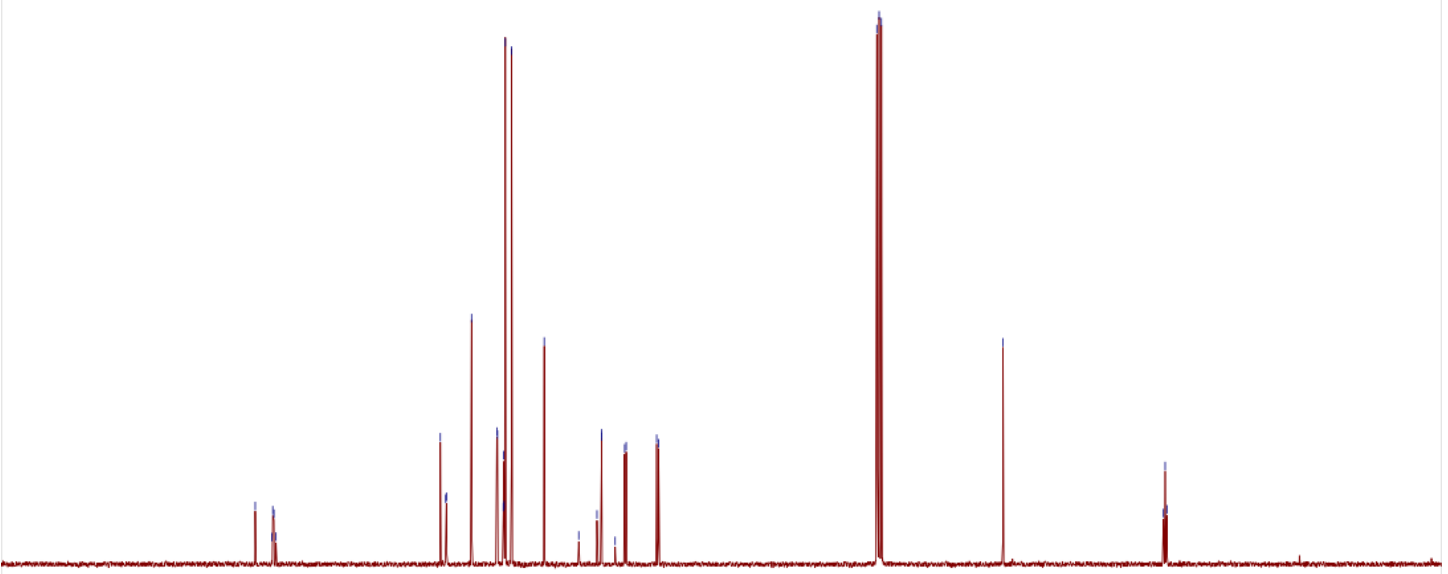

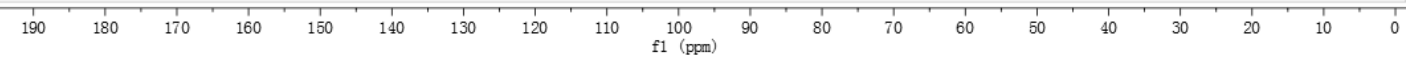


${ }^{1} \mathrm{H}$ NMR (400 MHz, CDCl $),{ }^{19} \mathrm{~F}\left\{{ }^{1} \mathrm{H}\right\}$ NMR $\left(375 \mathrm{MHz}, \mathrm{CDCl}_{3}\right),{ }^{13} \mathrm{C}\left\{{ }^{1} \mathrm{H}\right\}$ NMR (100 MHz, $\mathrm{CDCl}_{3}$ ) spectrum of product $4 \mathrm{f}$

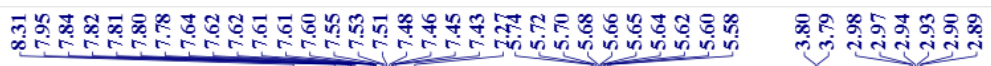

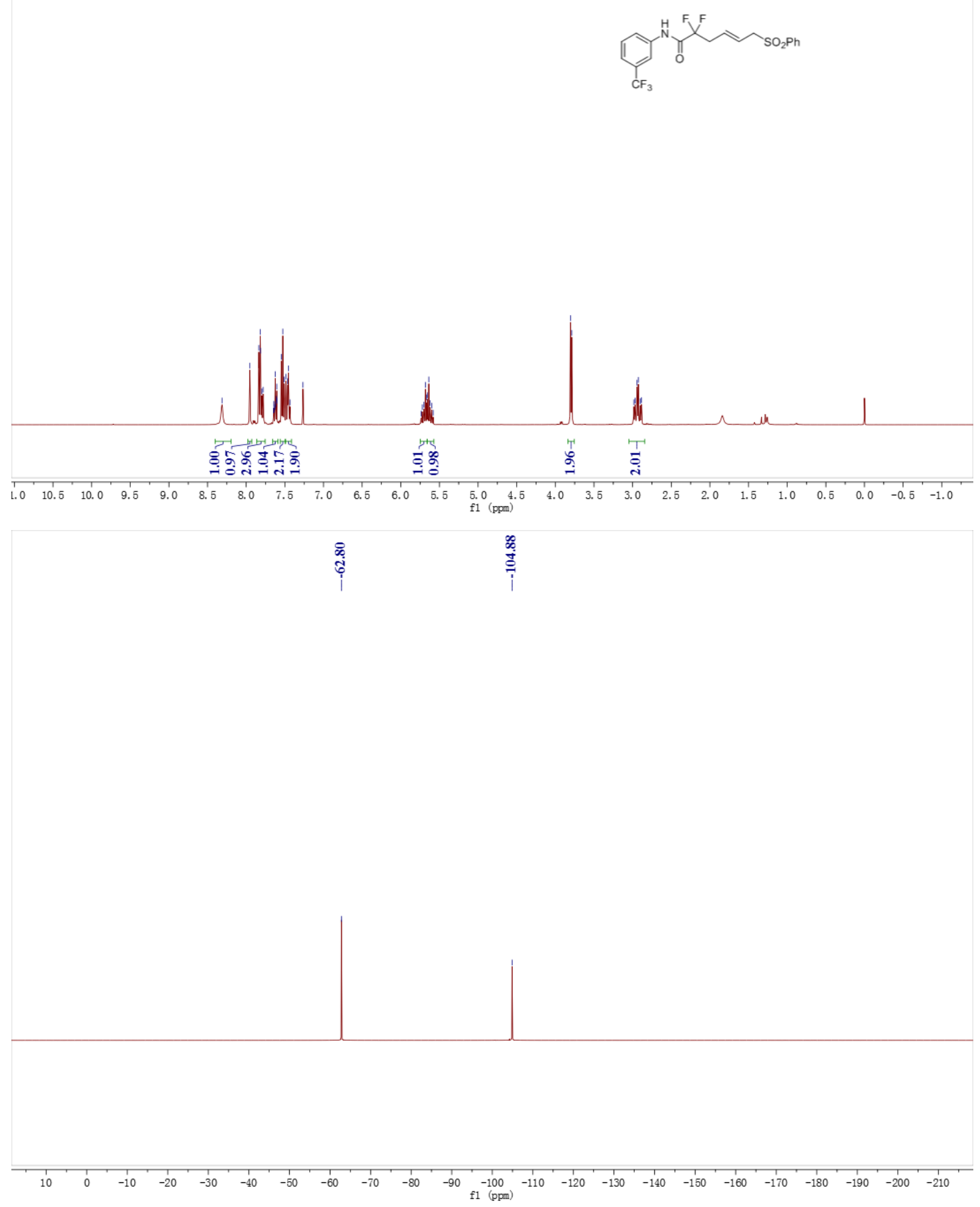




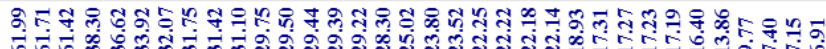

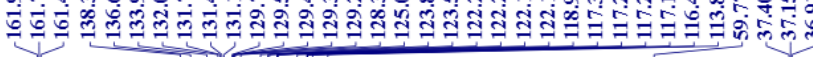

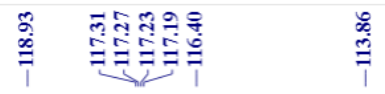
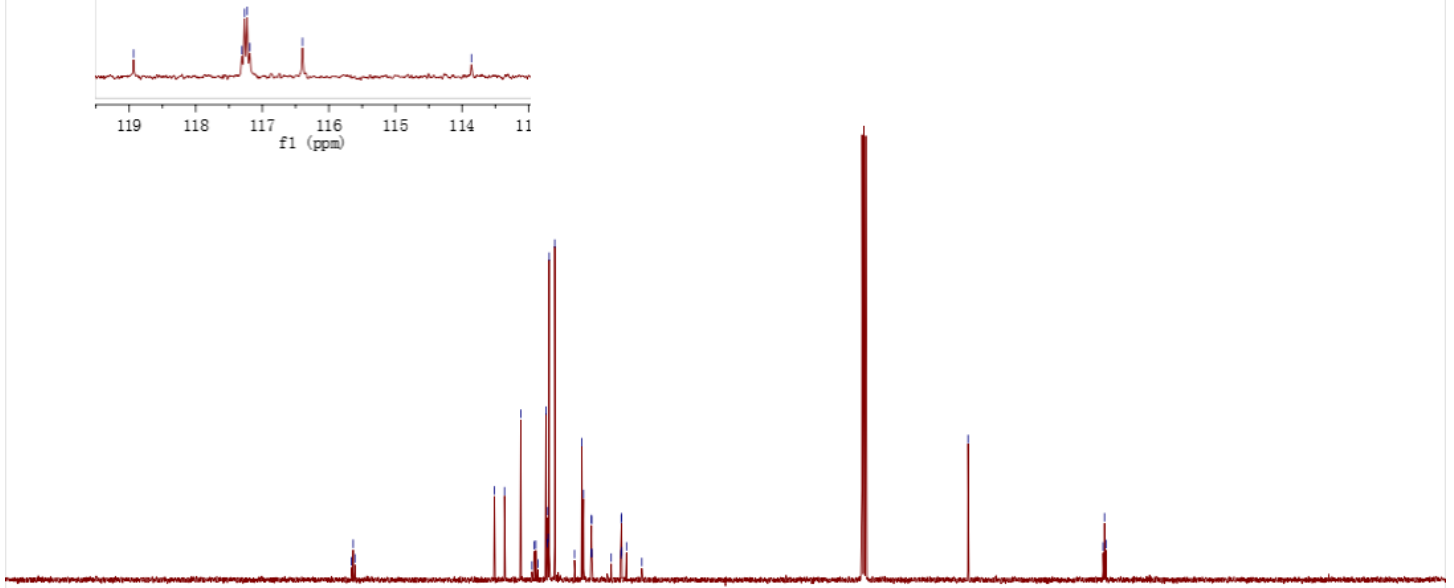

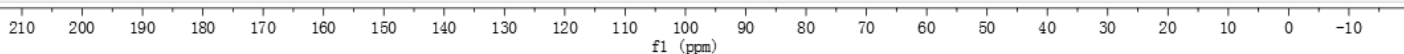


${ }^{1} \mathrm{H}$ NMR (400 MHz, DMSO-d $\left.d_{6}\right),{ }^{19} \mathrm{~F}\left\{{ }^{1} \mathrm{H}\right\}$ NMR (375 MHz, DMSO- $\left.d_{6}\right),{ }^{13} \mathrm{C}\left\{{ }^{1} \mathrm{H}\right\}$ NMR (100 MHz, DMSO- $d_{6}$ ) spectrum of product $4 \mathrm{~g}$

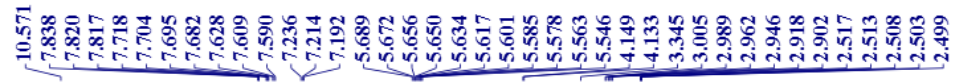
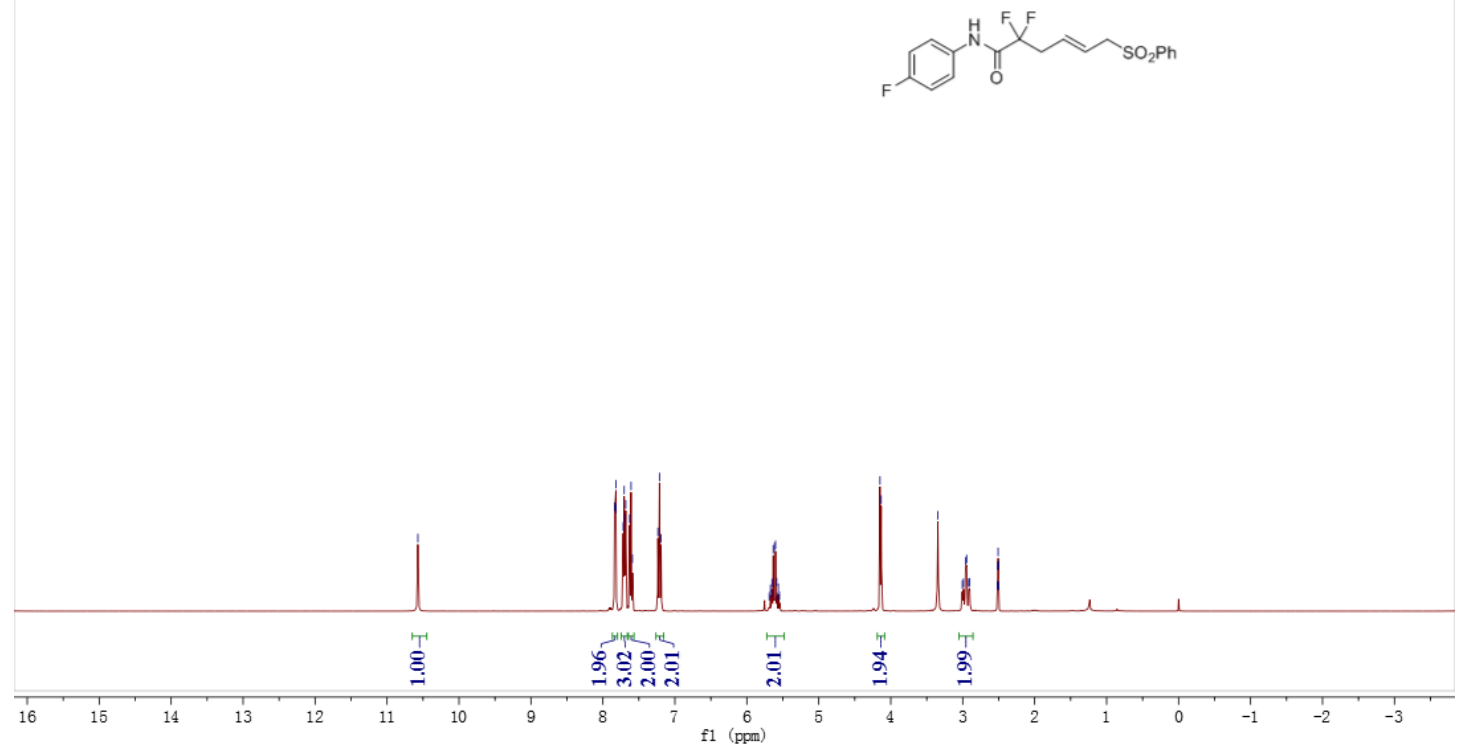

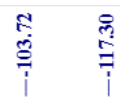
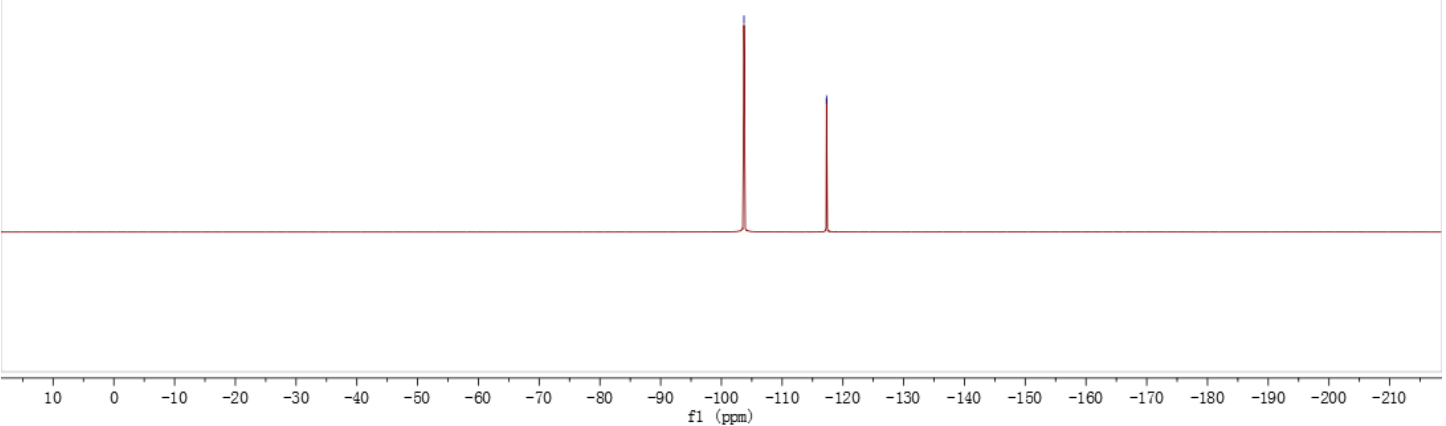


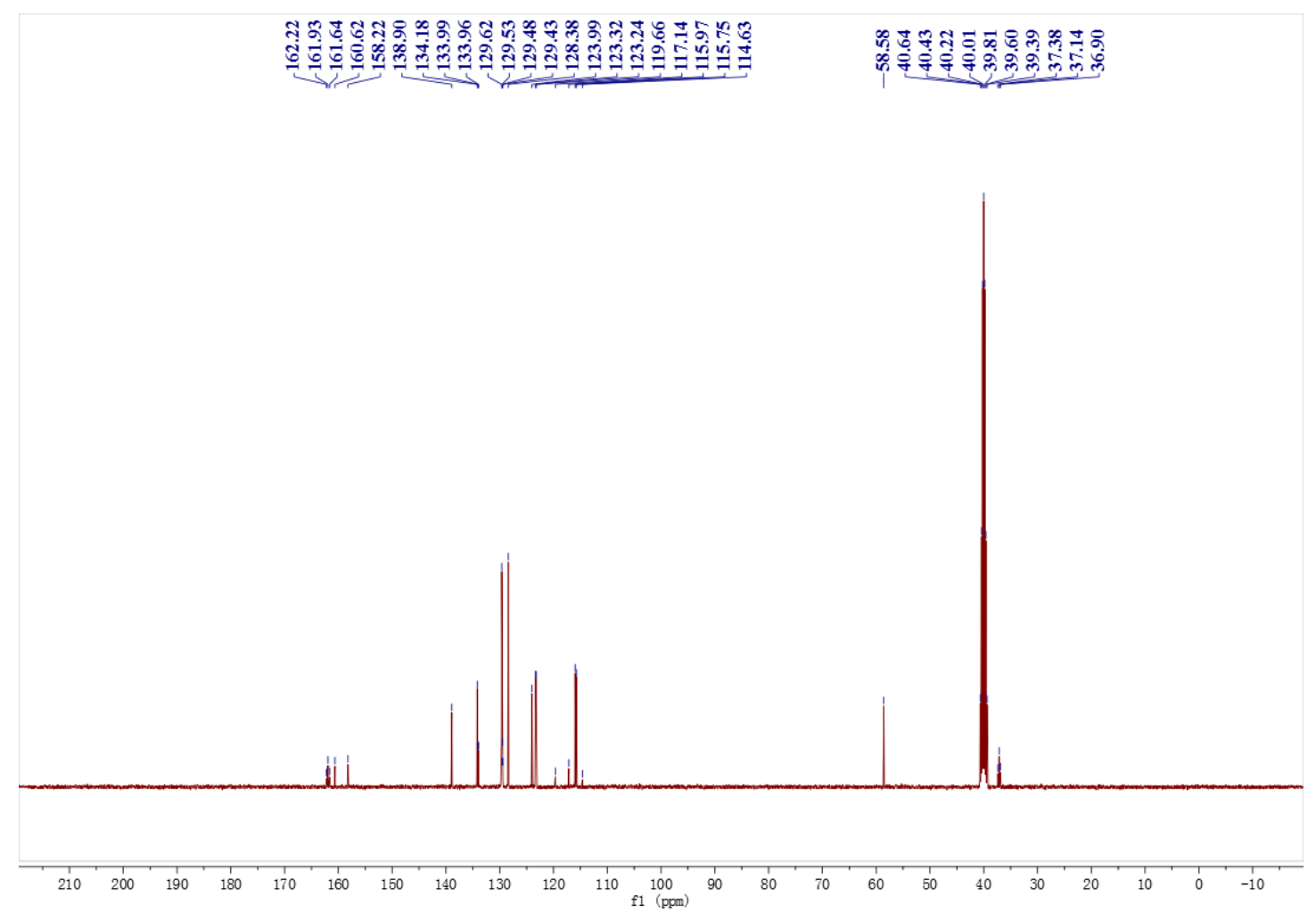


${ }^{1} \mathrm{H}$ NMR (400 MHz, DMSO-d $\left.d_{6}\right),{ }^{19} \mathrm{~F}\left\{{ }^{1} \mathrm{H}\right\}$ NMR (375 MHz, DMSO- $\left.d_{6}\right),{ }^{13} \mathrm{C}\left\{{ }^{1} \mathrm{H}\right\}$ NMR (100 MHz, DMSO- $d_{6}$ ) spectrum of product $4 \mathrm{~h}$

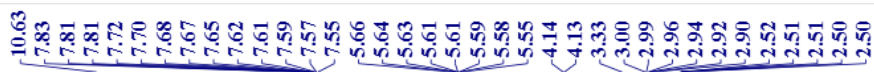

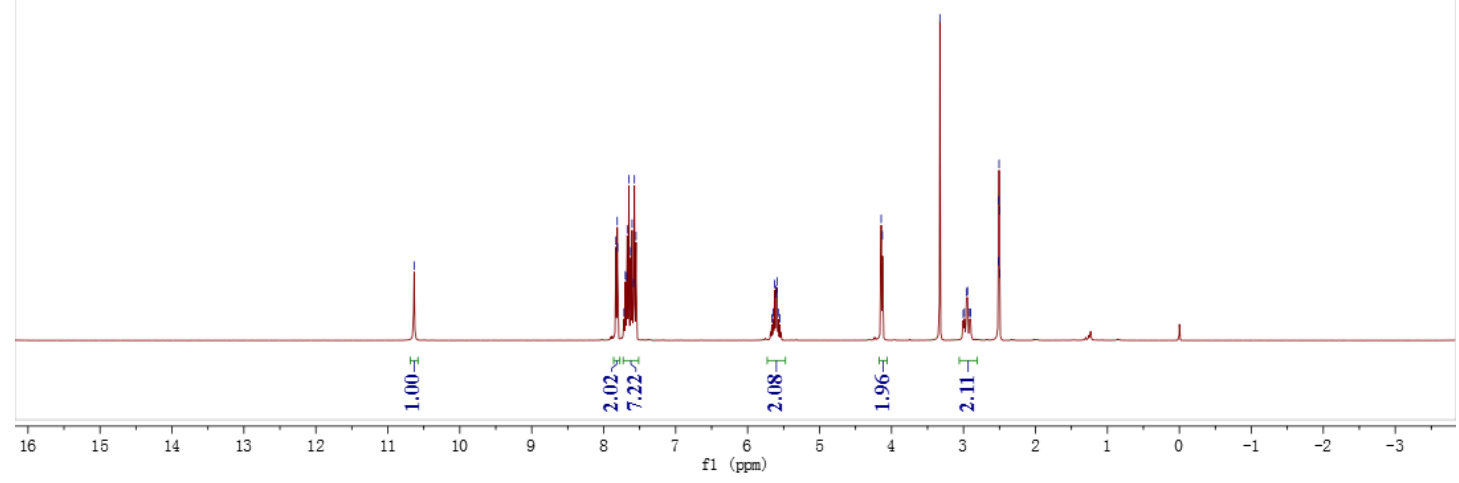
$\stackrel{8}{\stackrel{8}{\oplus}}$

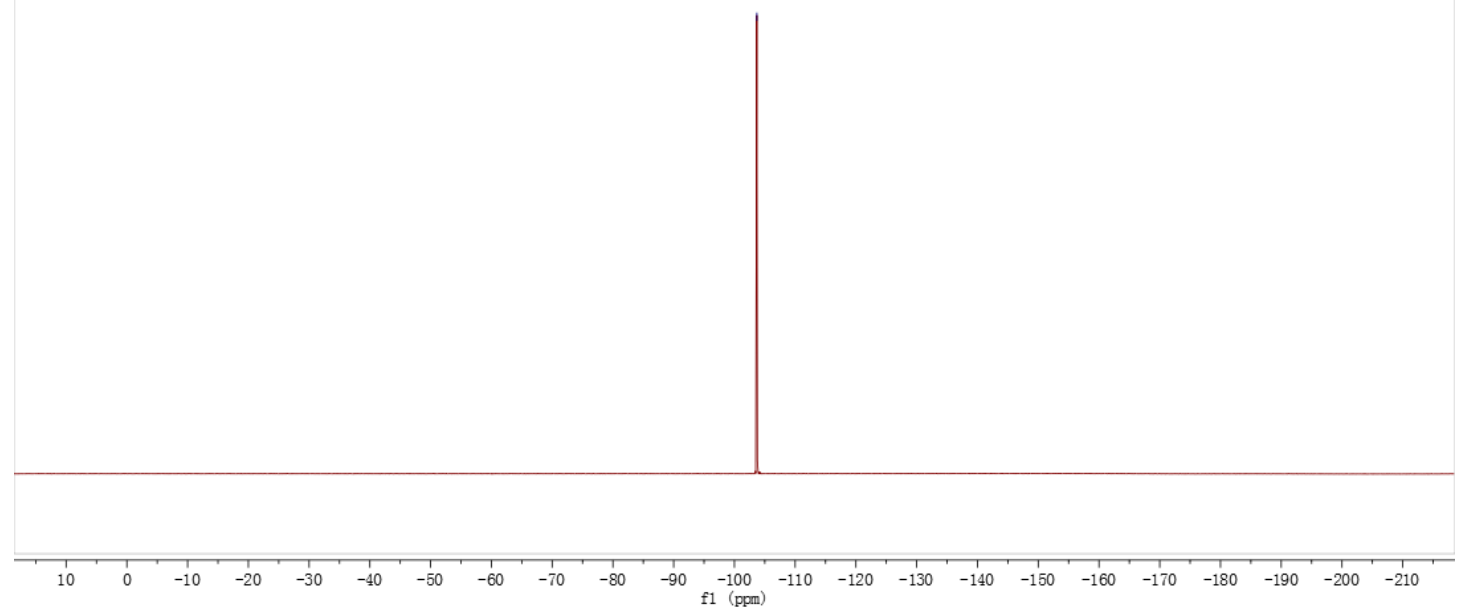




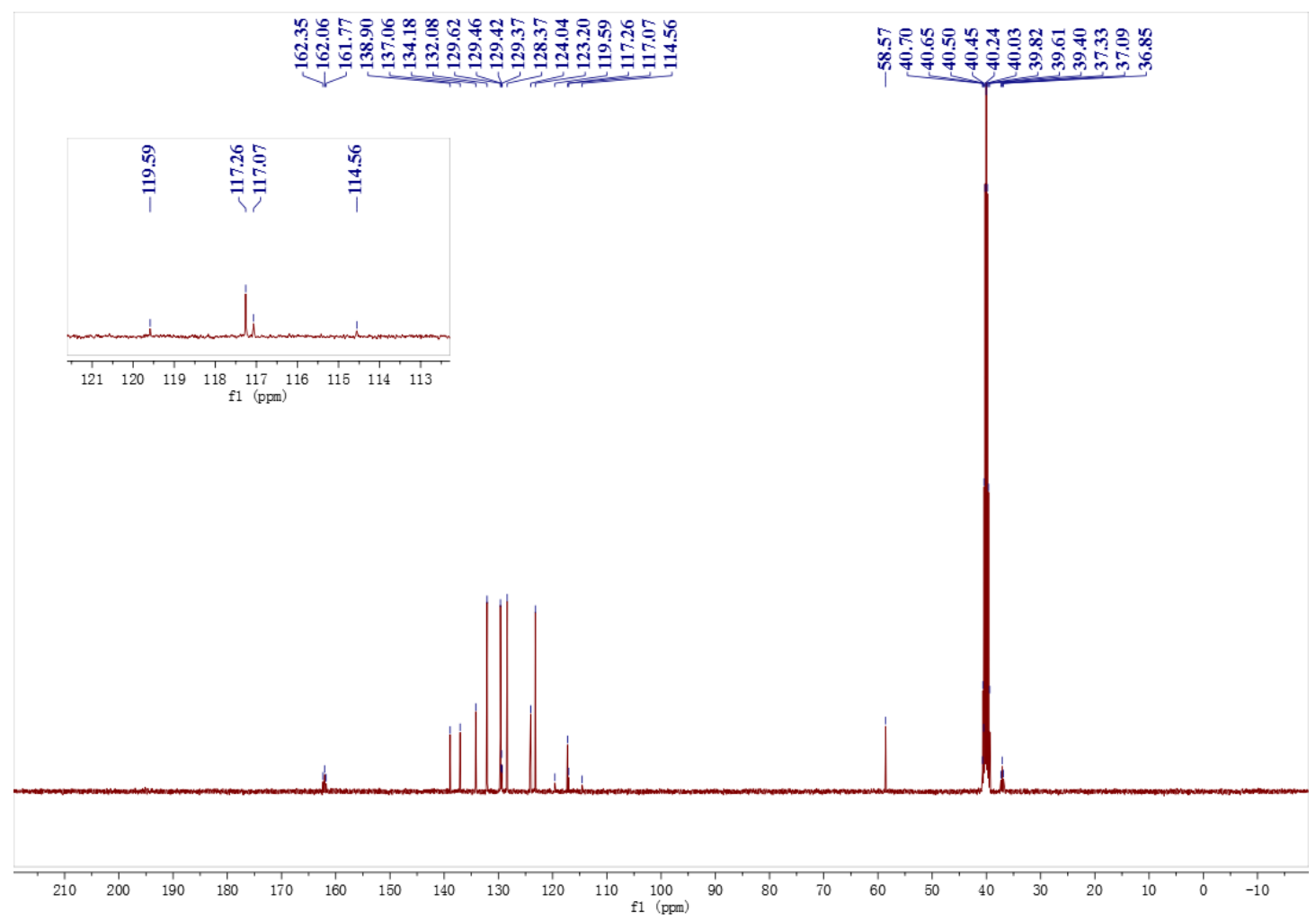


${ }^{1} \mathrm{H}$ NMR (400 MHz, DMSO-d $\left.d_{6}\right),{ }^{19} \mathrm{~F}\left\{{ }^{1} \mathrm{H}\right\}$ NMR (375 MHz, DMSO- $\left.d_{6}\right),{ }^{13} \mathrm{C}\left\{{ }^{1} \mathrm{H}\right\}$ NMR (100 MHz, DMSO- $d_{6}$ ) spectrum of product $4 \mathrm{i}$

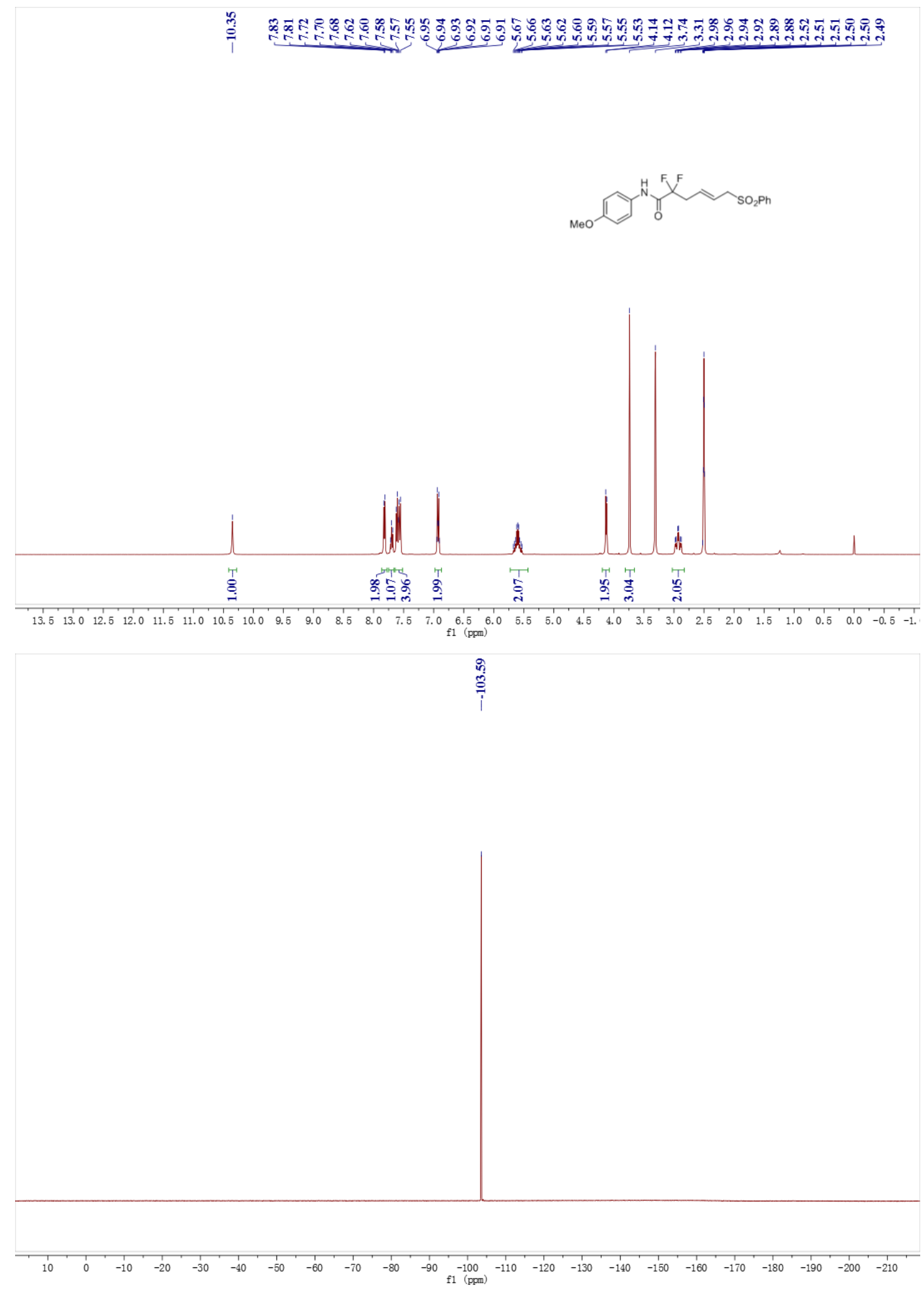




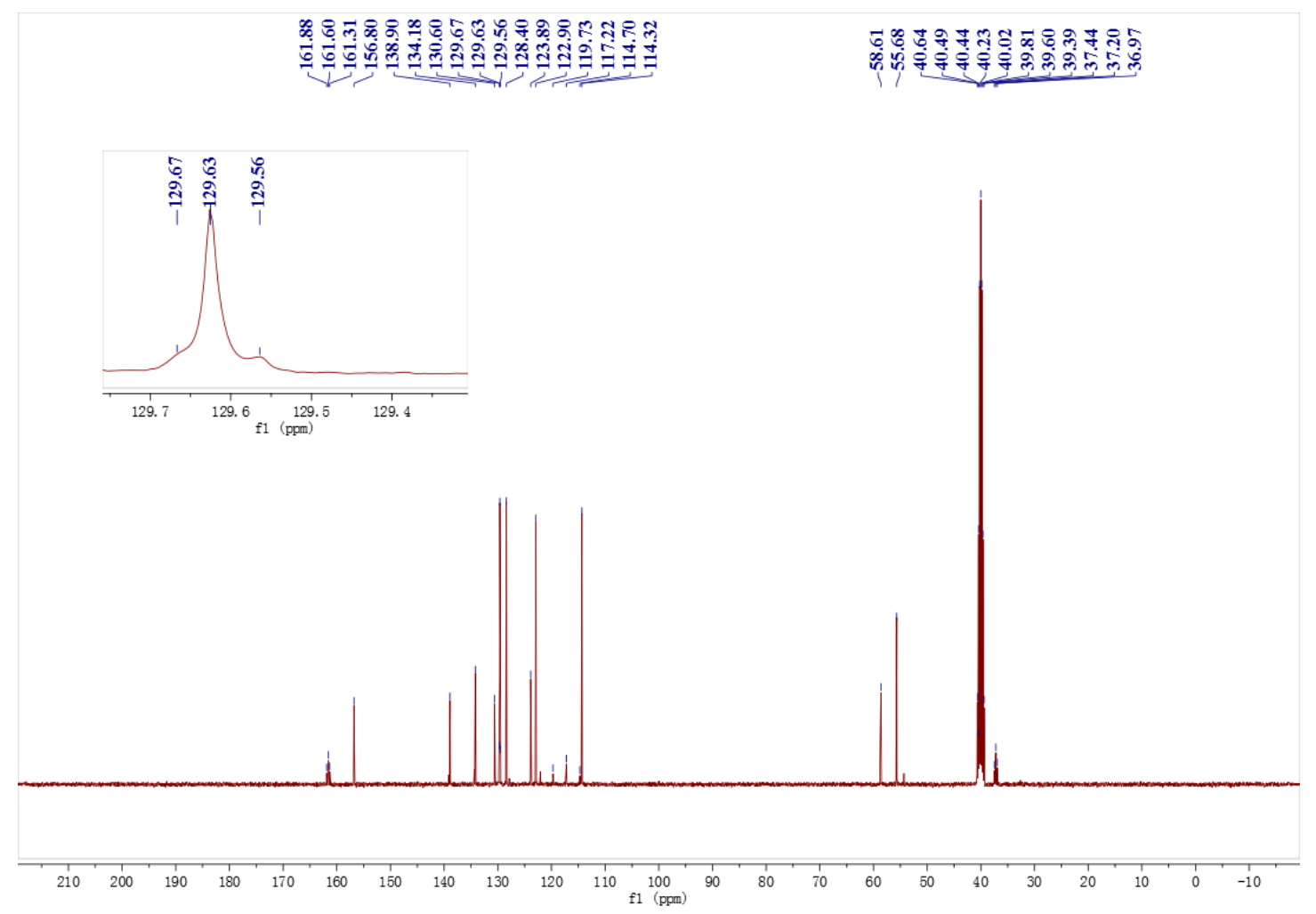

S46 
${ }^{1} \mathrm{H}$ NMR (400 MHz, CDCl $),{ }^{19} \mathrm{~F}\left\{{ }^{1} \mathrm{H}\right\}$ NMR $\left(375 \mathrm{MHz}, \mathrm{CDCl}_{3}\right),{ }^{13} \mathrm{C}\left\{{ }^{1} \mathrm{H}\right\}$ NMR (100 MHz, $\mathrm{CDCl}_{3}$ ) spectrum of product $4 \mathrm{j}$

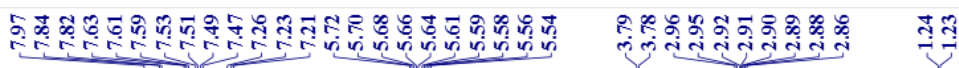
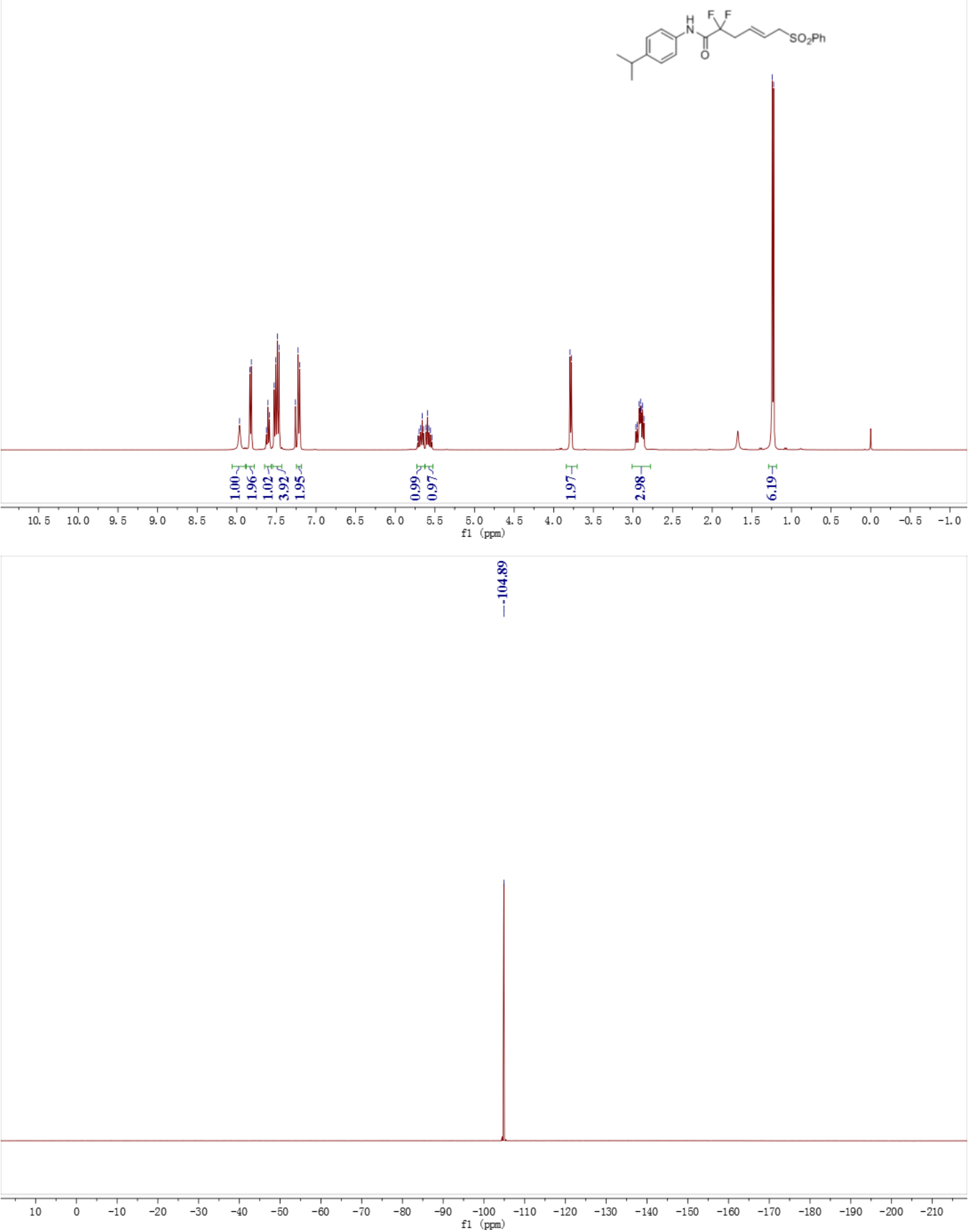


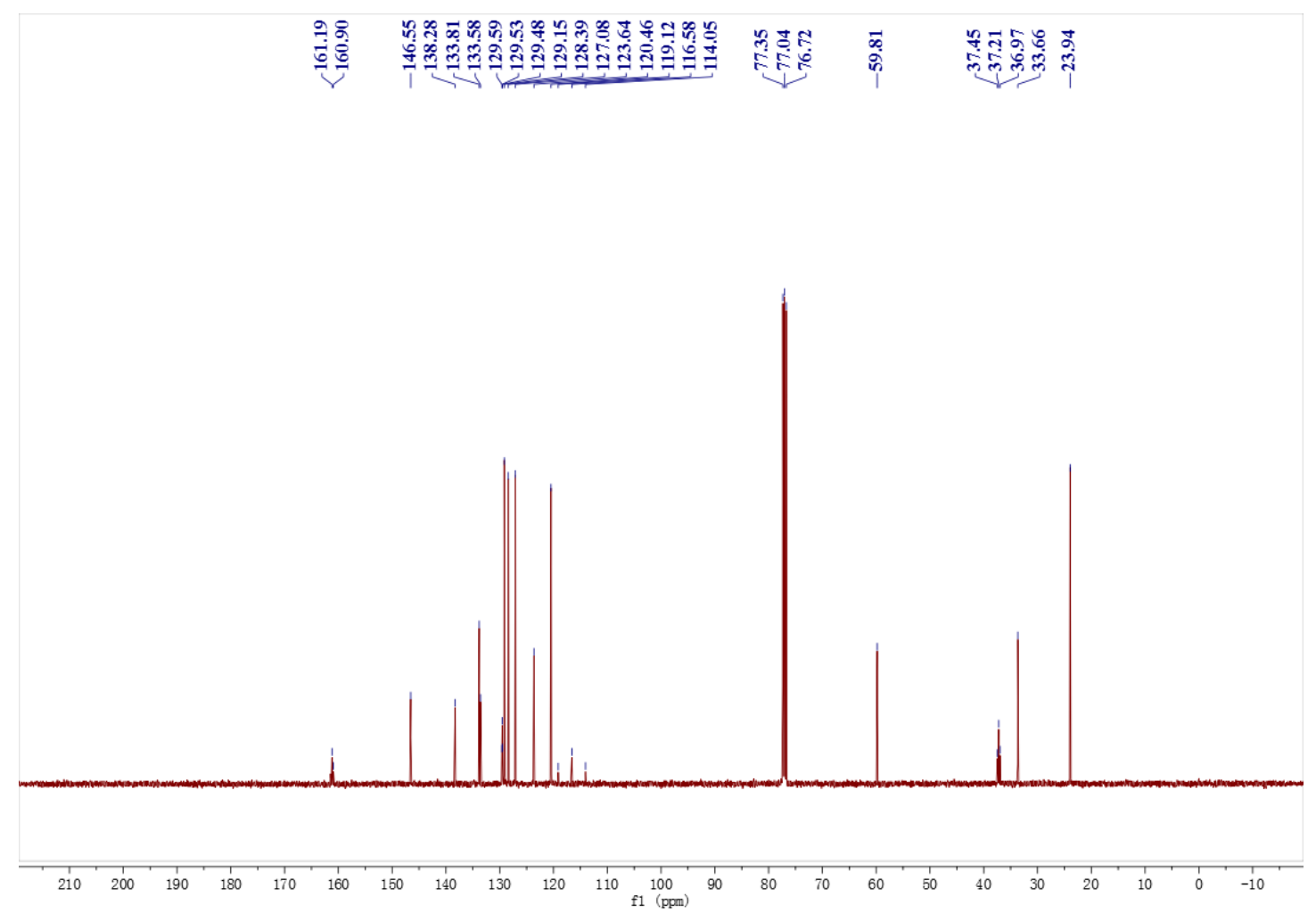


${ }^{1} \mathrm{H}$ NMR (400 MHz, CDCl $),{ }^{19} \mathrm{~F}\left\{{ }^{1} \mathrm{H}\right\}$ NMR $\left(375 \mathrm{MHz}, \mathrm{CDCl}_{3}\right),{ }^{13} \mathrm{C}\left\{{ }^{1} \mathrm{H}\right\}$ NMR (100 MHz, $\mathrm{CDCl}_{3}$ ) spectrum of product $4 \mathrm{k}$

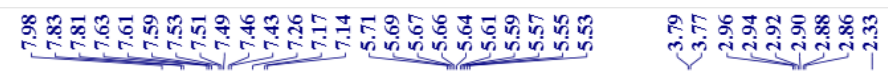

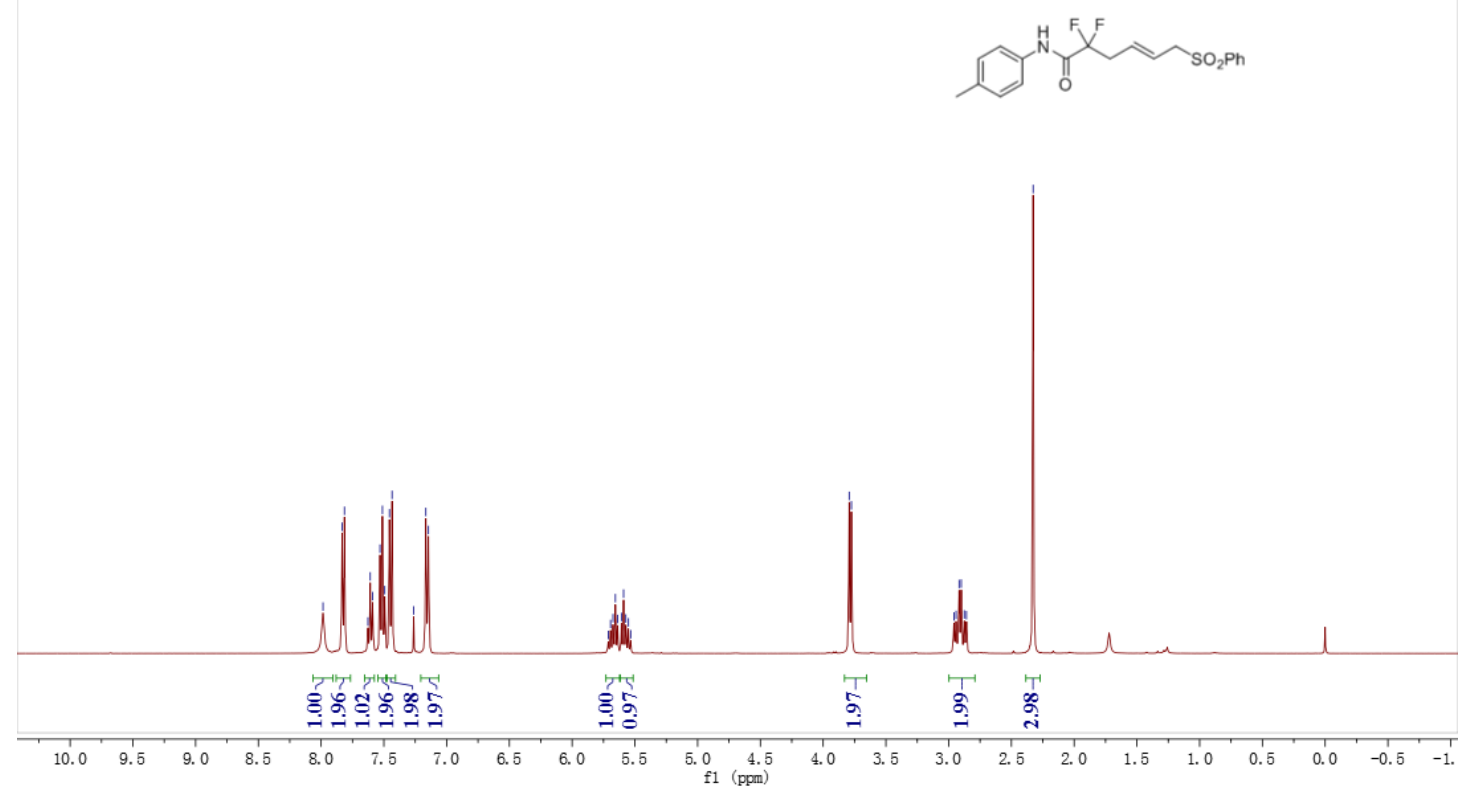

$\stackrel{8}{\frac{1}{2}}$

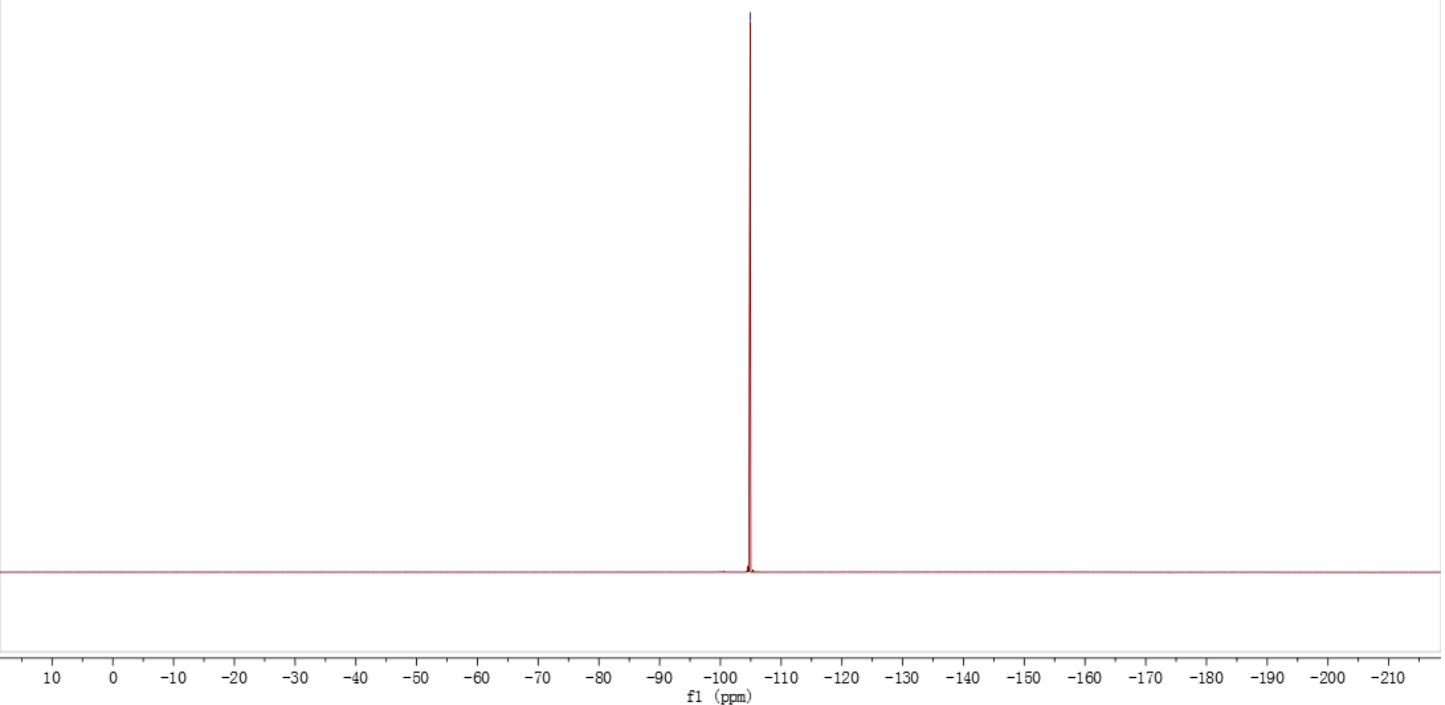




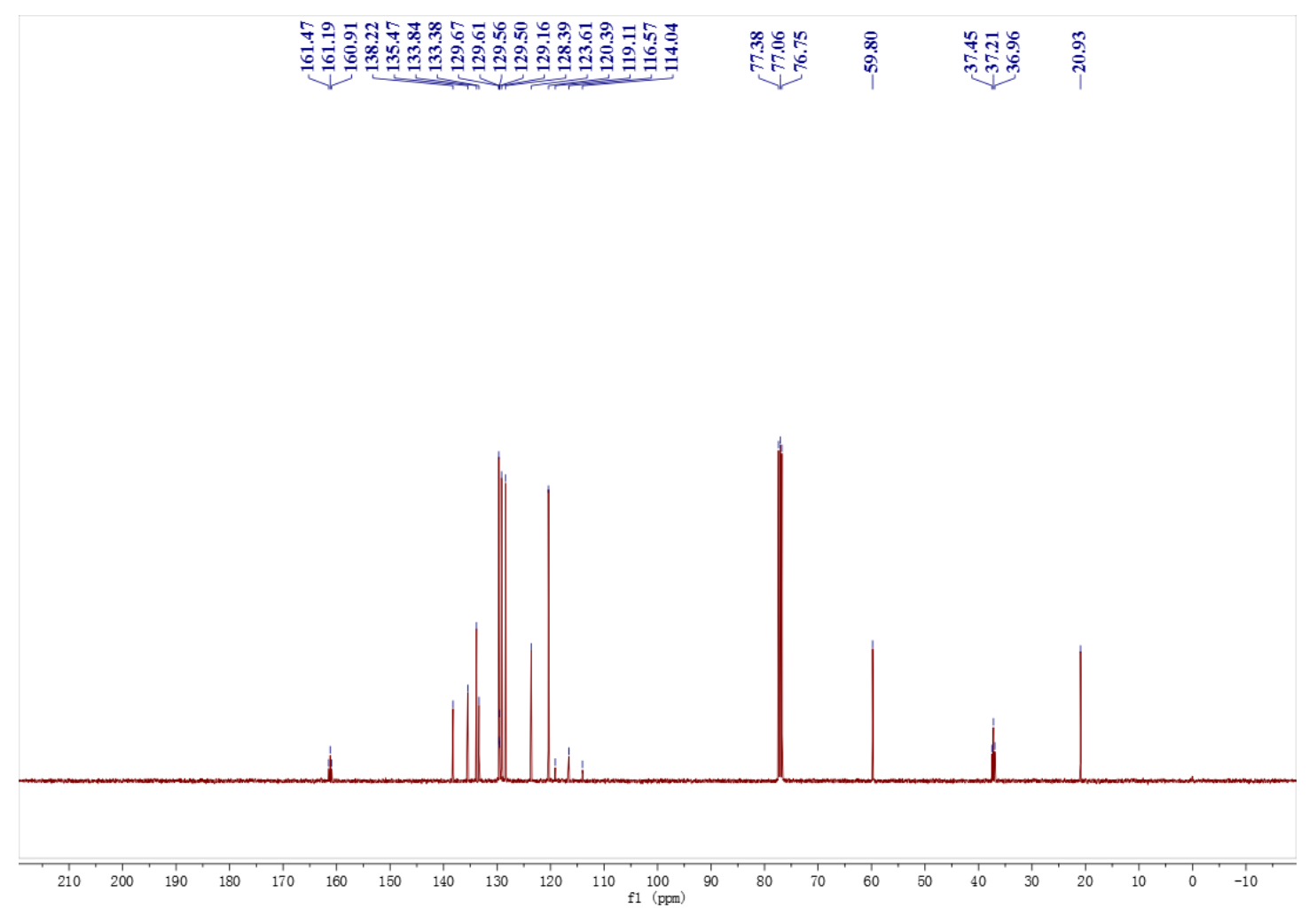


${ }^{1} \mathrm{H}$ NMR $\left(400 \mathrm{MHz}, \mathrm{CDCl}_{3}\right),{ }^{19} \mathrm{~F}\left\{{ }^{1} \mathrm{H}\right\}$ NMR $\left(375 \mathrm{MHz}, \mathrm{CDCl}_{3}\right),{ }^{13} \mathrm{C}\left\{{ }^{1} \mathrm{H}\right\}$ NMR $(100 \mathrm{MHz}$, $\mathrm{CDCl}_{3}$ ) spectrum of product 41

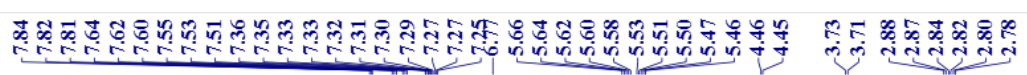
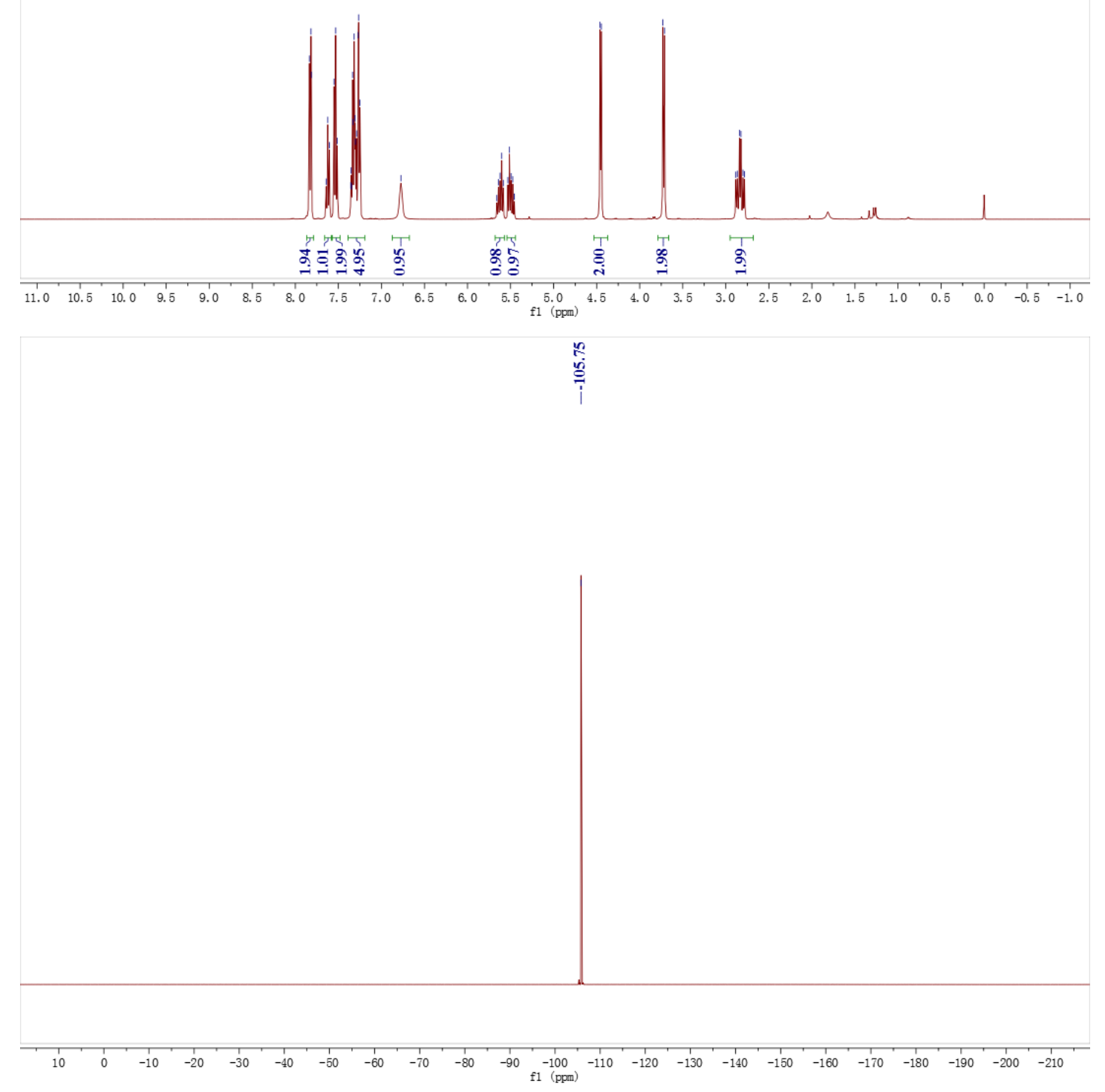

S51 


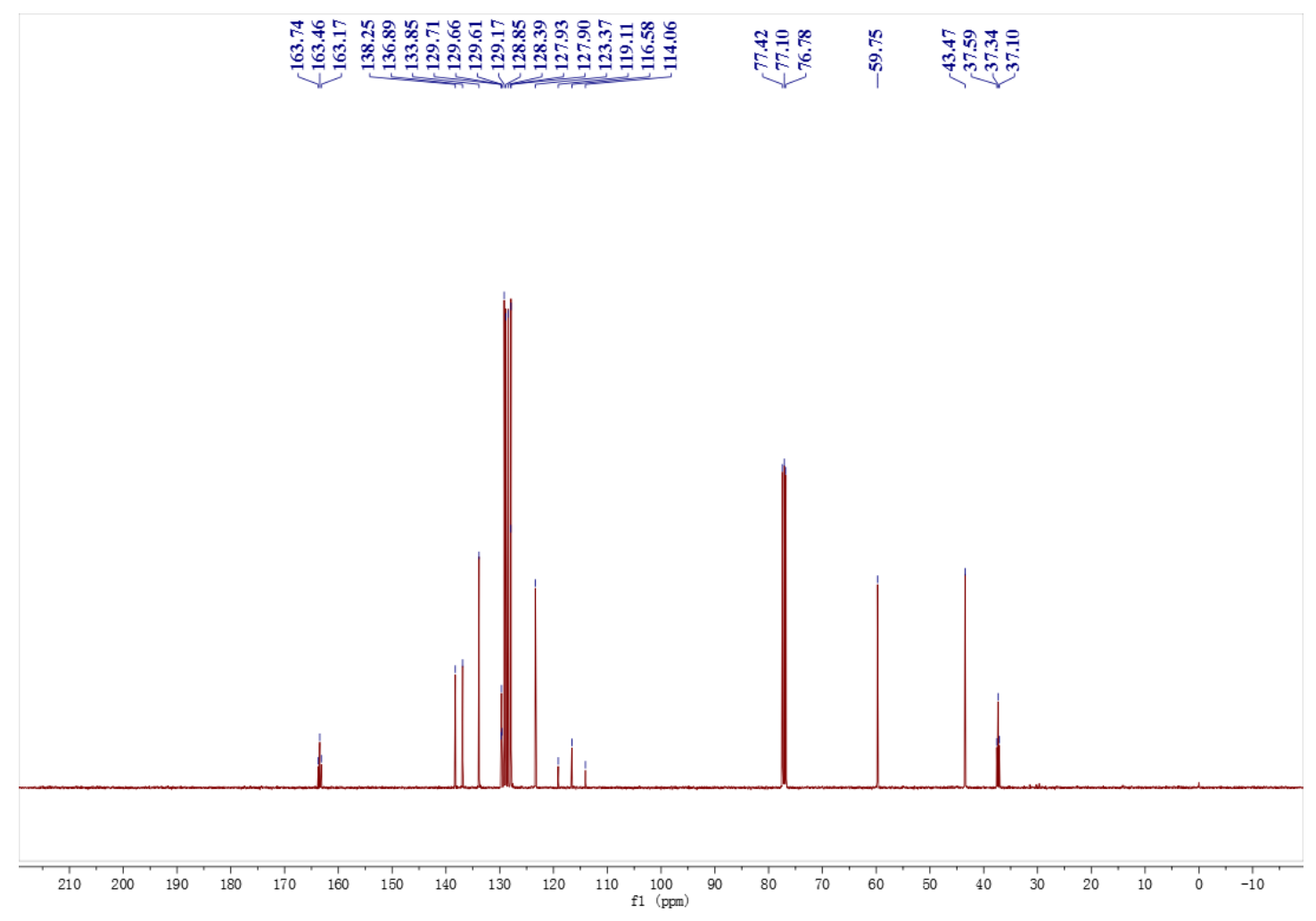


${ }^{1} \mathrm{H}$ NMR (400 MHz, CDCl $),{ }^{19} \mathrm{~F}\left\{{ }^{1} \mathrm{H}\right\}$ NMR $\left(375 \mathrm{MHz}, \mathrm{CDCl}_{3}\right),{ }^{13} \mathrm{C}\left\{{ }^{1} \mathrm{H}\right\}$ NMR (100 MHz, $\mathrm{CDCl}_{3}$ ) spectrum of product $4 \mathrm{~m}$

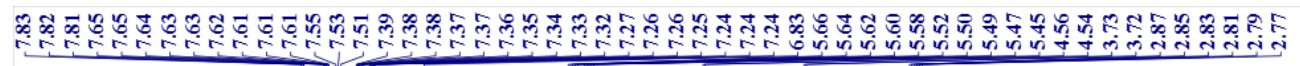
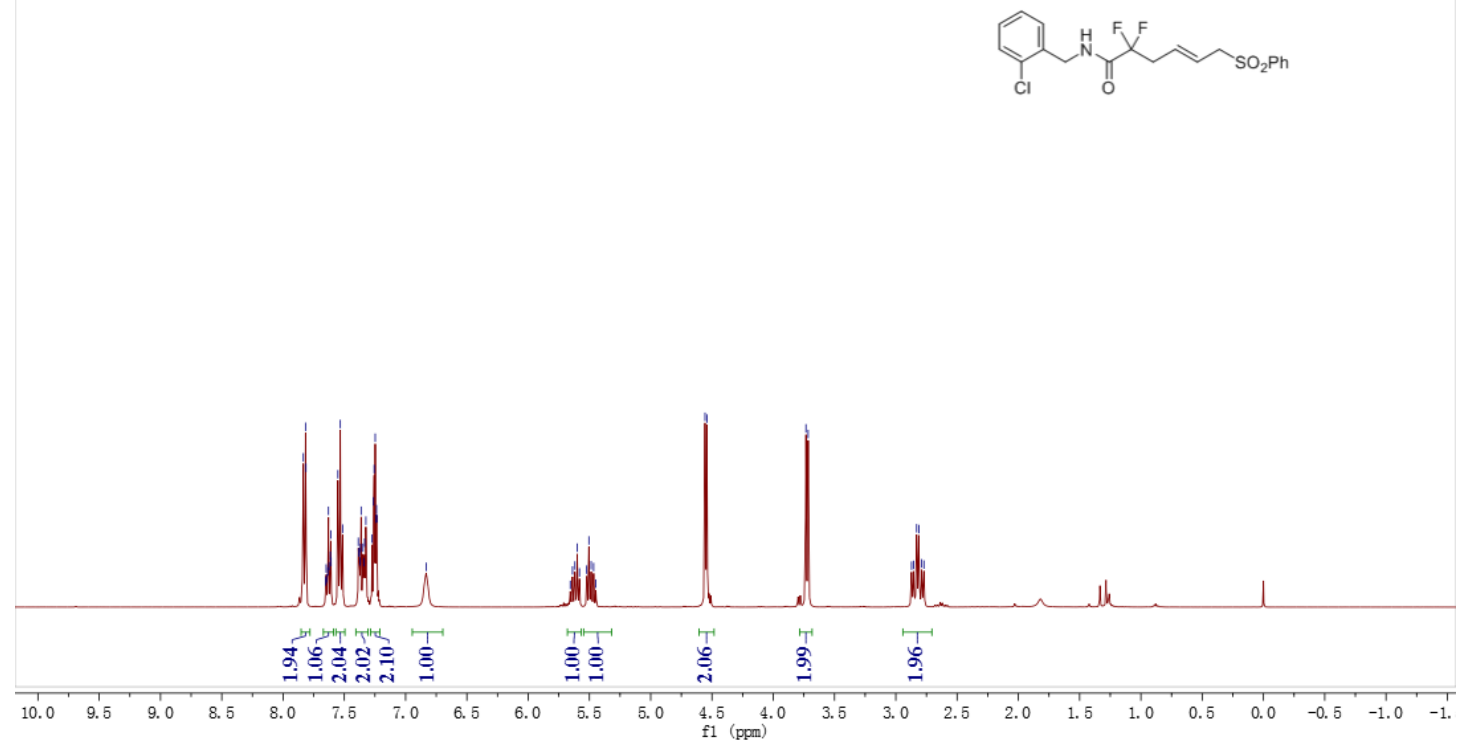
$\stackrel{\infty}{\stackrel{0}{0}}$

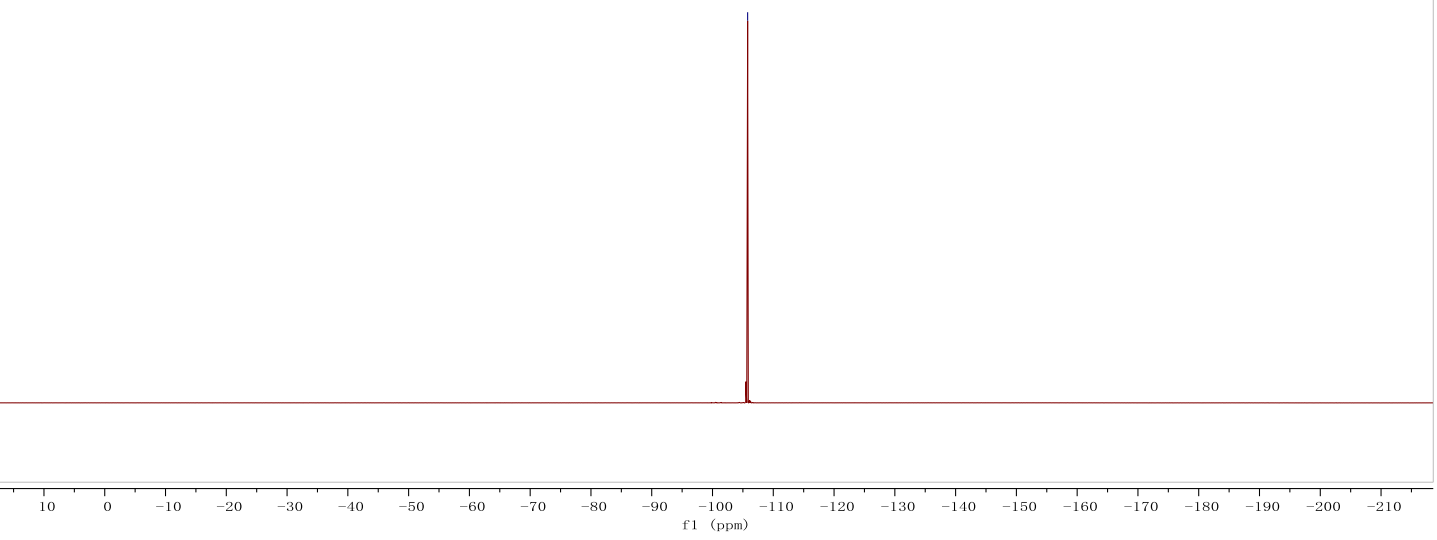




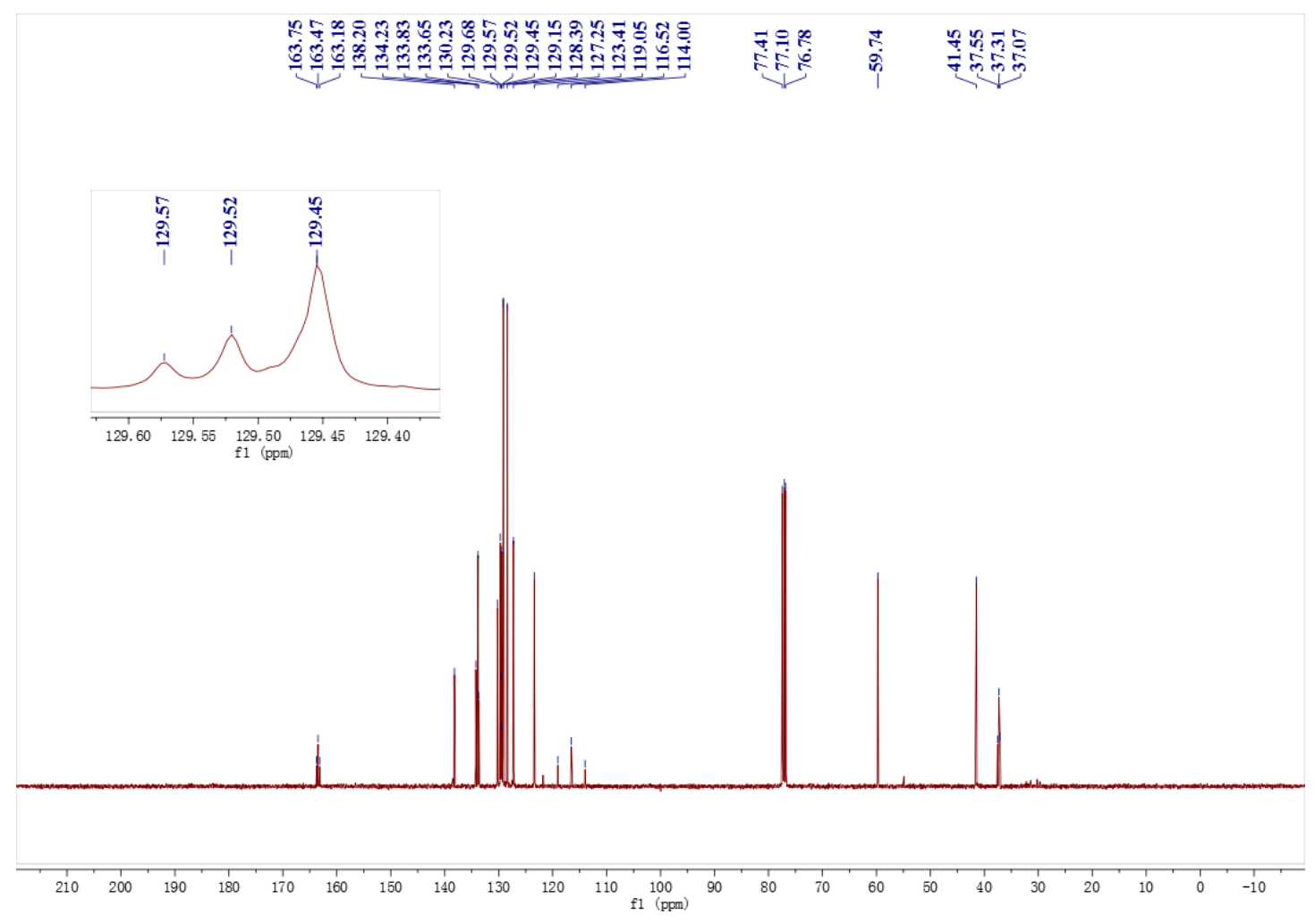


${ }^{1} \mathrm{H}$ NMR (400 MHz, CDCl $),{ }^{19} \mathrm{~F}\left\{{ }^{1} \mathrm{H}\right\}$ NMR $\left(375 \mathrm{MHz}, \mathrm{CDCl}_{3}\right),{ }^{13} \mathrm{C}\left\{{ }^{1} \mathrm{H}\right\}$ NMR (100 MHz, $\mathrm{CDCl}_{3}$ ) spectrum of product $4 \mathrm{n}$

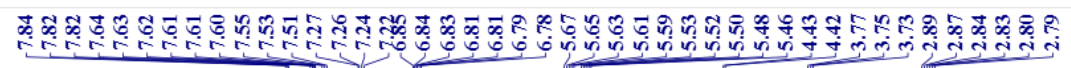

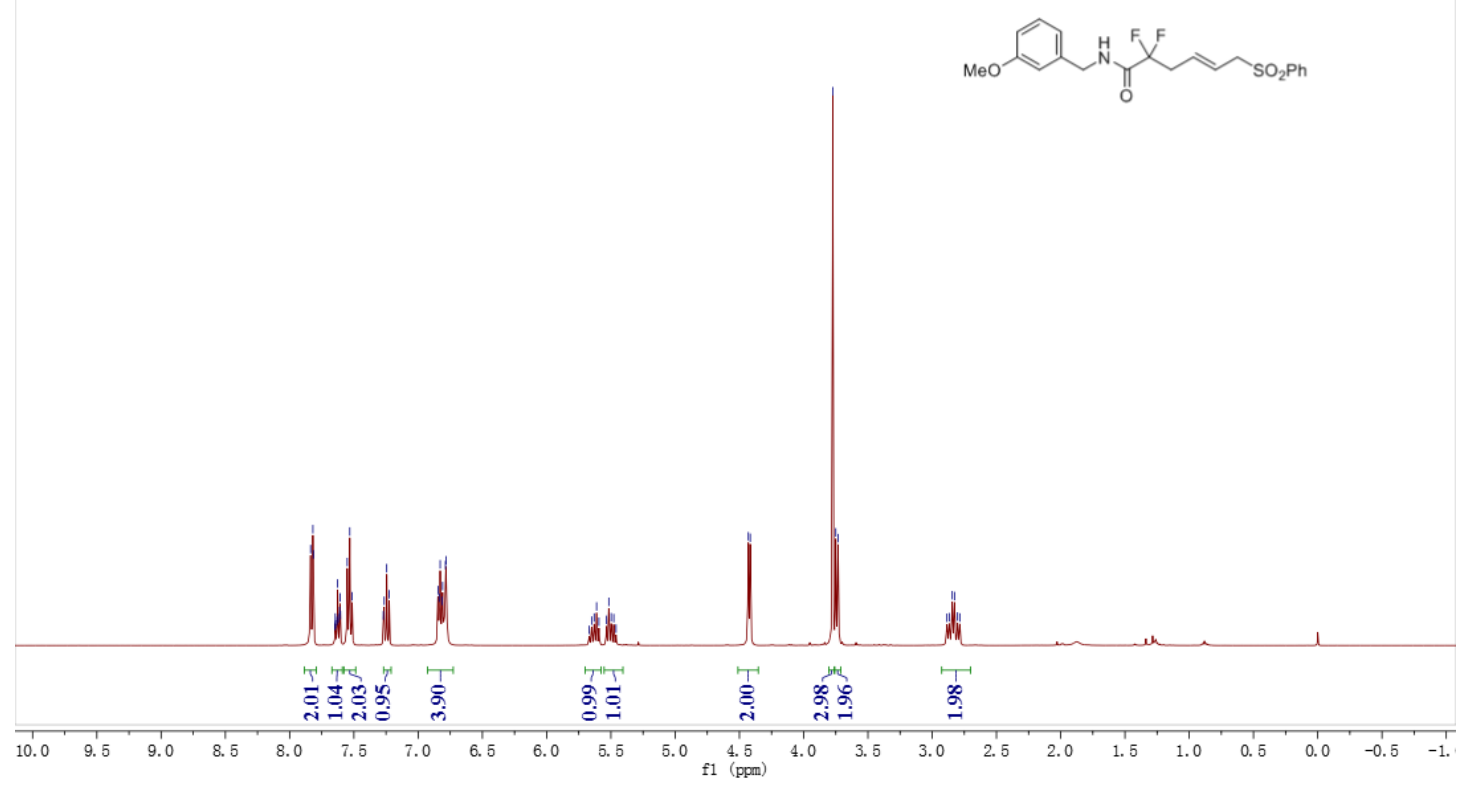

है

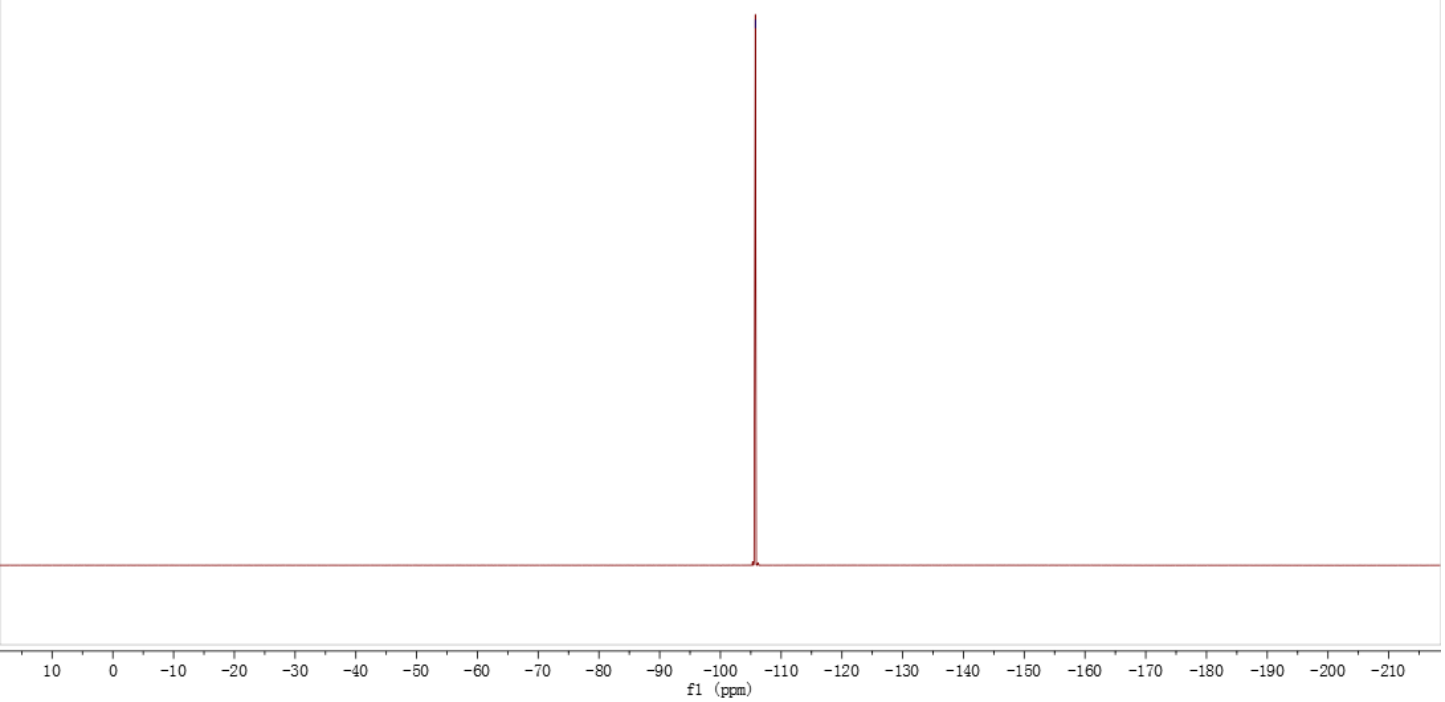




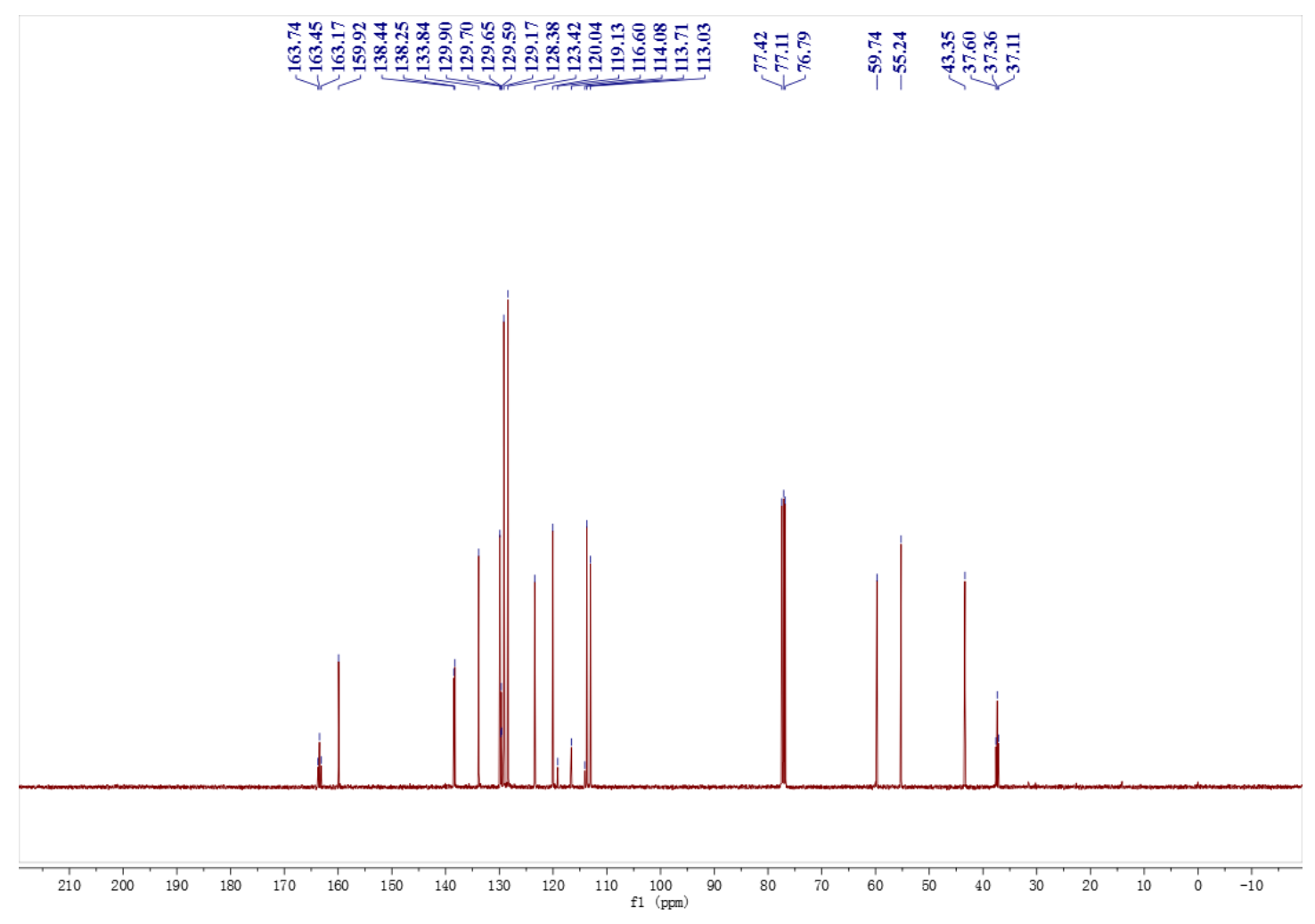


${ }^{1} \mathrm{H}$ NMR (400 MHz, DMSO-d $\left.d_{6}\right),{ }^{19} \mathrm{~F}\left\{{ }^{1} \mathrm{H}\right\}$ NMR (375 MHz, DMSO- $\left.d_{6}\right),{ }^{13} \mathrm{C}\left\{{ }^{1} \mathrm{H}\right\}$ NMR (100 MHz, DMSO- $d_{6}$ ) spectrum of product 40

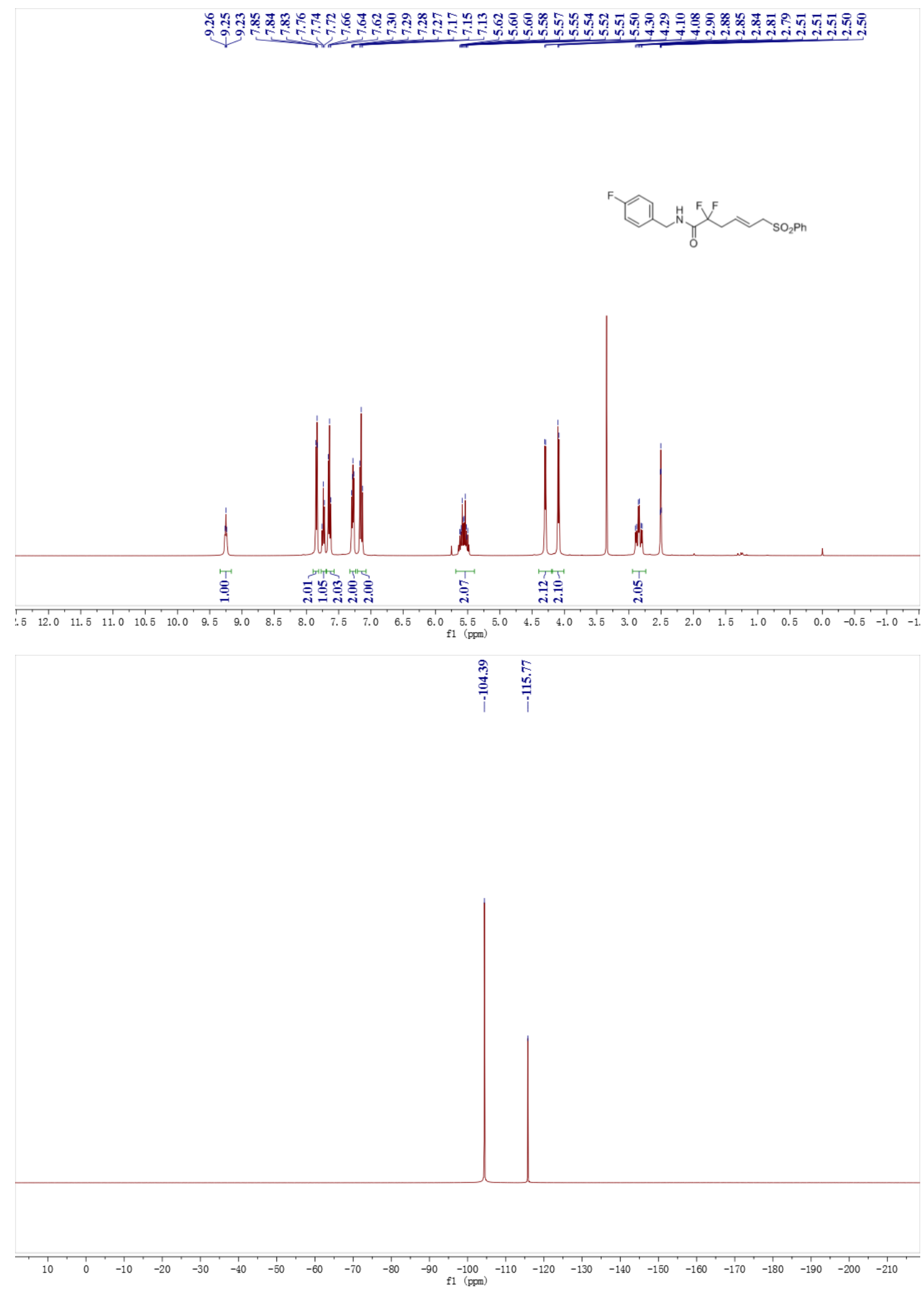




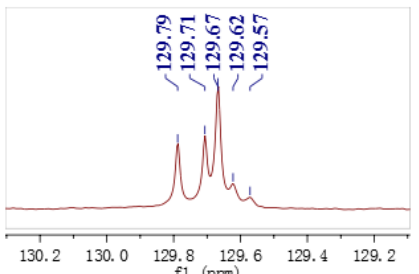

$\begin{array}{llllll}130.2 & 130.0 & 129.8 & 129.6 & 129.4 & 129.2\end{array}$

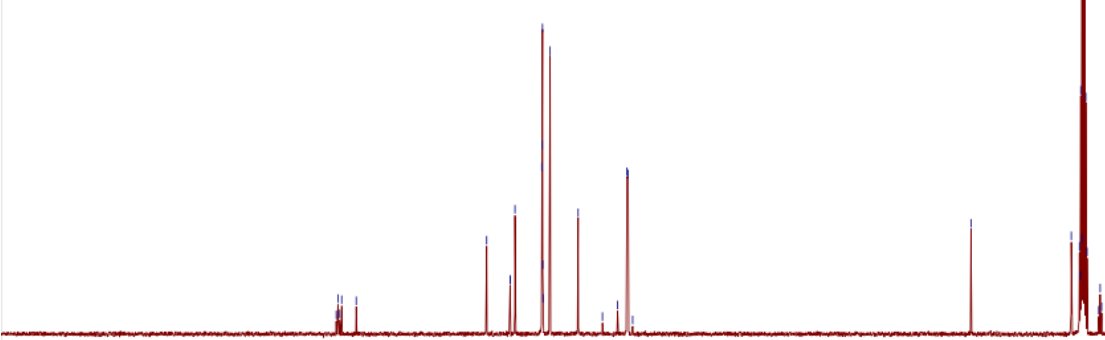

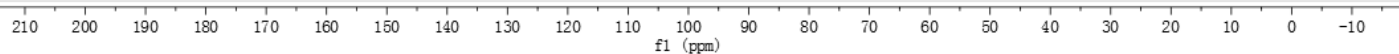


${ }^{1} \mathrm{H}$ NMR (400 MHz, CDCl $),{ }^{19} \mathrm{~F}\left\{{ }^{1} \mathrm{H}\right\}$ NMR $\left(375 \mathrm{MHz}, \mathrm{CDCl}_{3}\right),{ }^{13} \mathrm{C}\left\{{ }^{1} \mathrm{H}\right\}$ NMR (100 MHz, $\left.\mathrm{CDCl}_{3}\right)$ spectrum of product $4 \mathrm{p}$

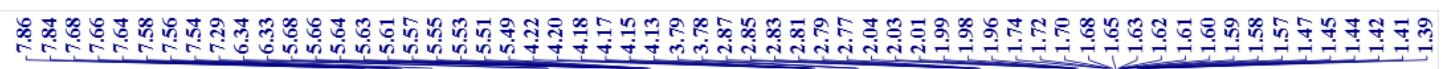
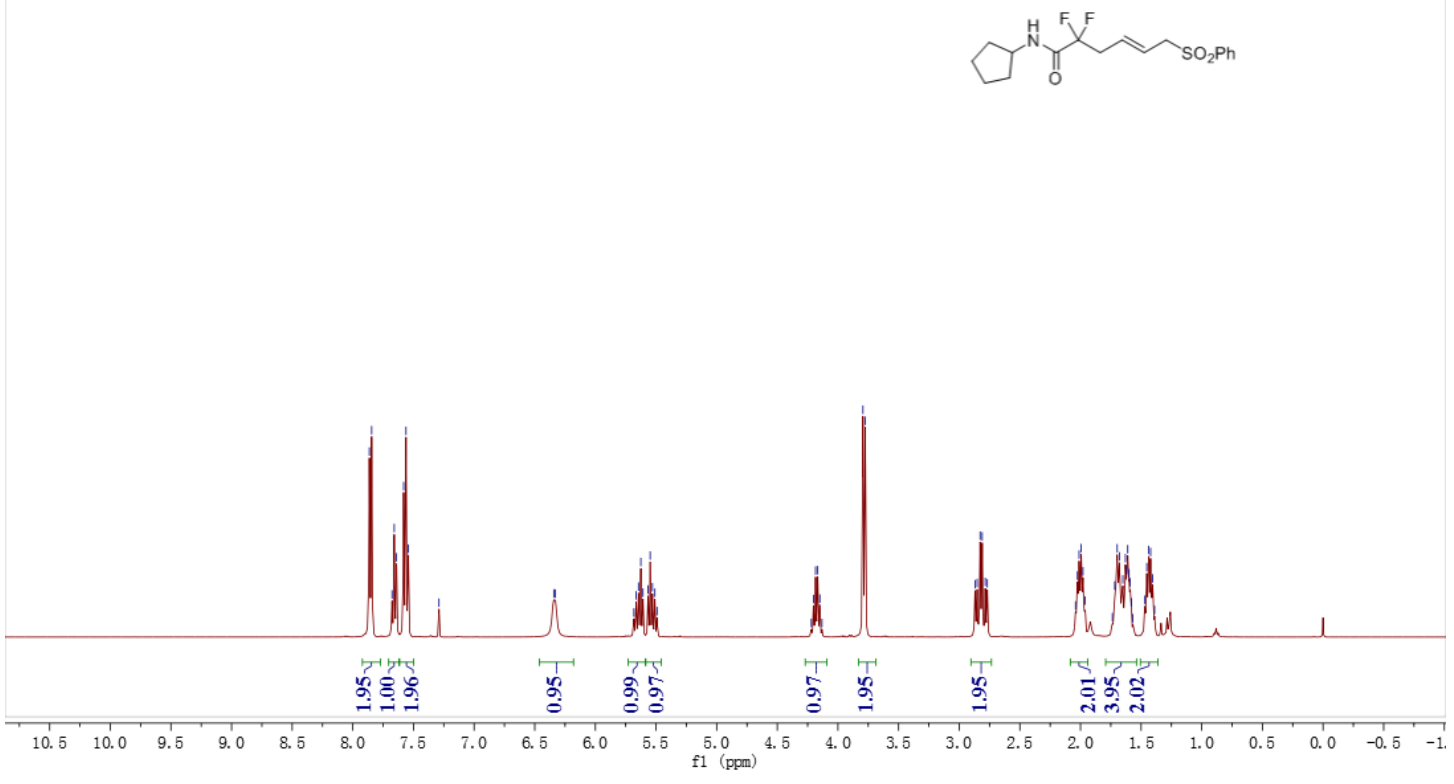

$\infty$
$\stackrel{\infty}{0}$
$\stackrel{0}{\mid}$

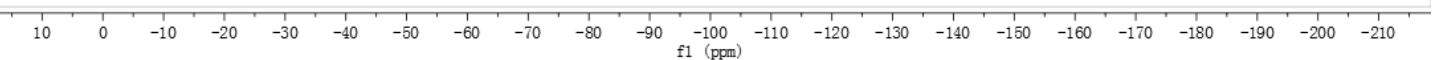




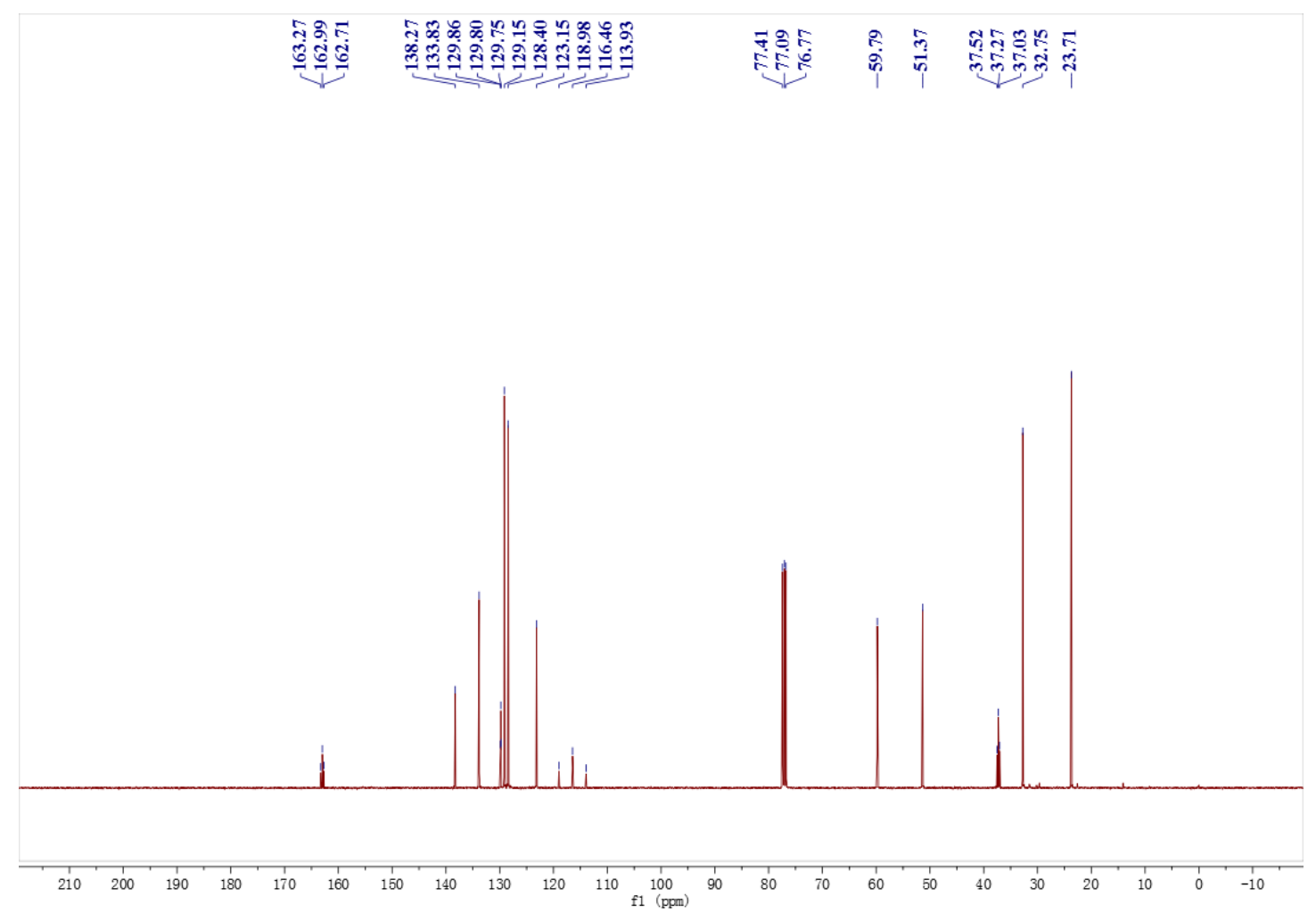


${ }^{1} \mathrm{H}$ NMR (400 MHz, CDCl $),{ }^{19} \mathrm{~F}\left\{{ }^{1} \mathrm{H}\right\}$ NMR $\left(375 \mathrm{MHz}, \mathrm{CDCl}_{3}\right),{ }^{13} \mathrm{C}\left\{{ }^{1} \mathrm{H}\right\}$ NMR (100 MHz, $\mathrm{CDCl}_{3}$ ) spectrum of product $4 \mathrm{q}$

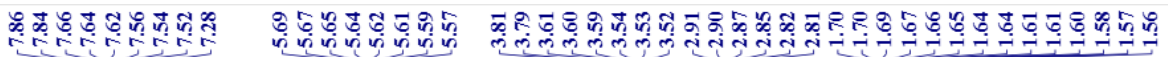
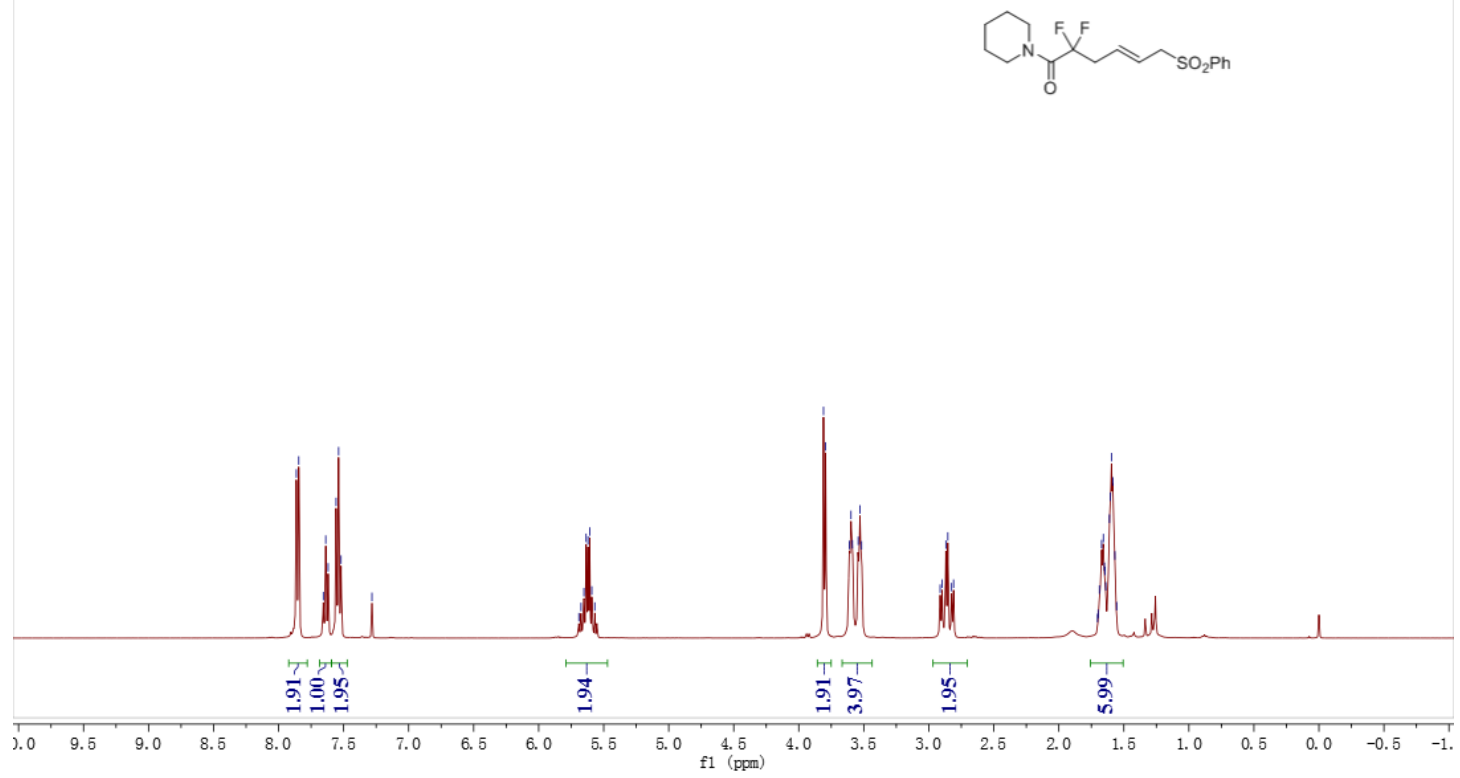

จ

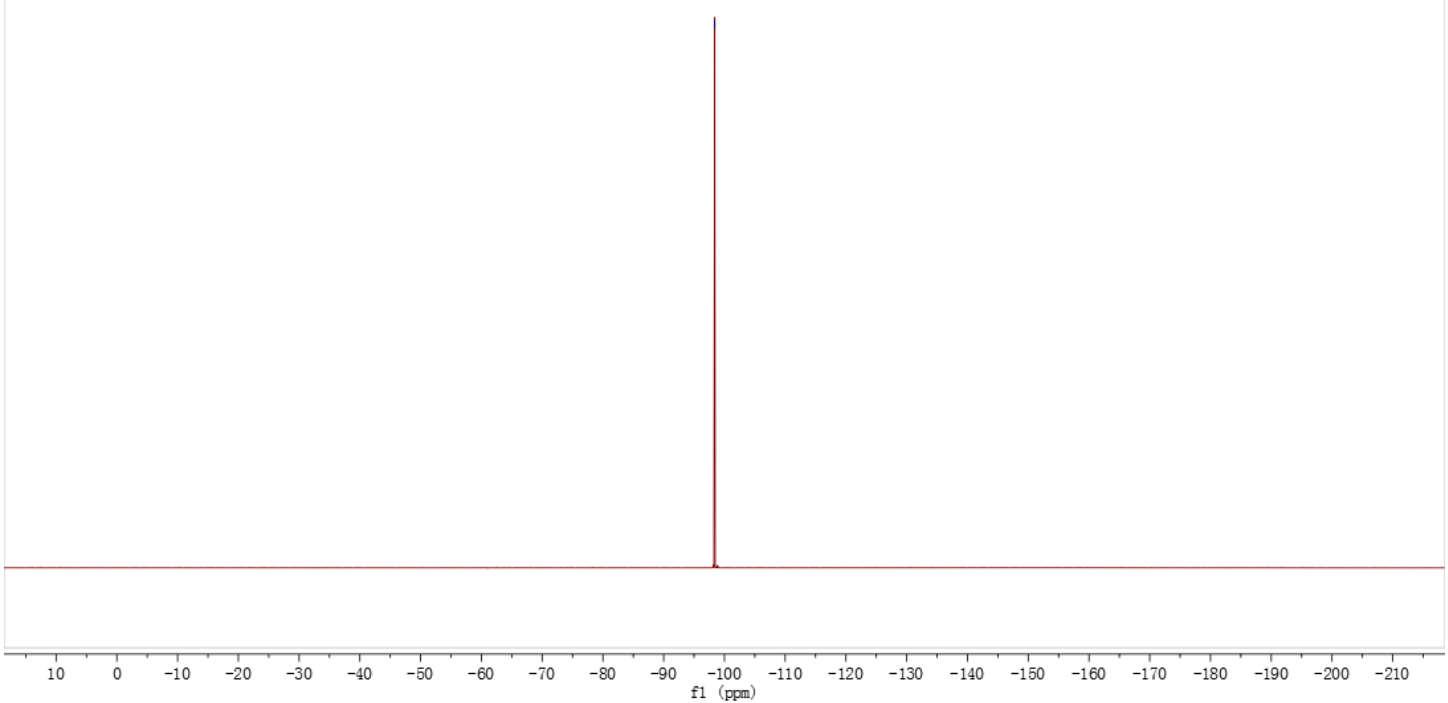




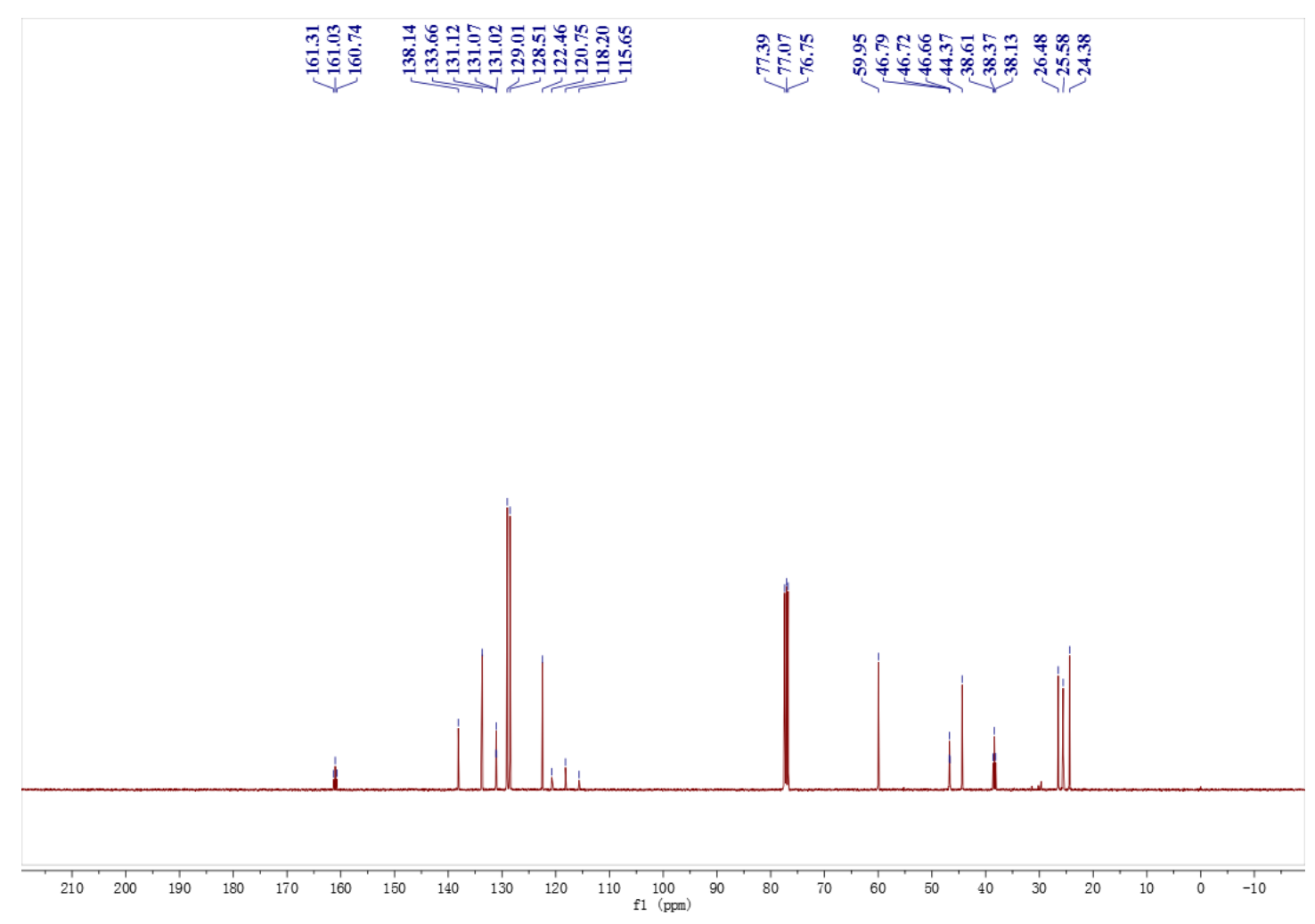


${ }^{1} \mathrm{H}$ NMR (400 MHz, CDCl $),{ }^{19} \mathrm{~F}\left\{{ }^{1} \mathrm{H}\right\}$ NMR $\left(375 \mathrm{MHz}, \mathrm{CDCl}_{3}\right),{ }^{13} \mathrm{C}\left\{{ }^{1} \mathrm{H}\right\}$ NMR (100 MHz, $\left.\mathrm{CDCl}_{3}\right)$ spectrum of product $4 \mathrm{r}$

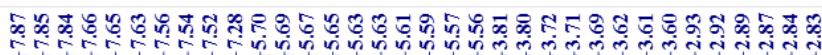
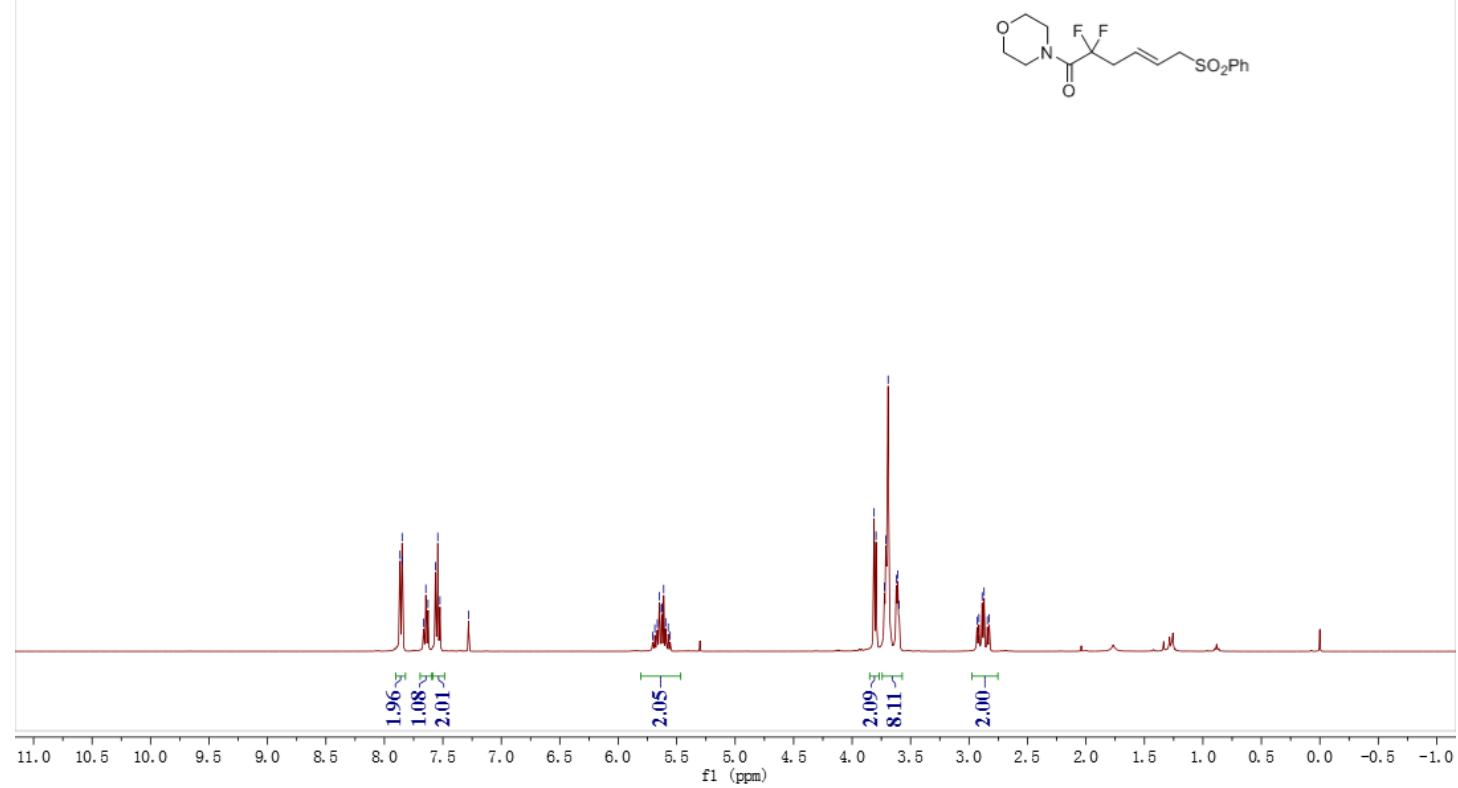

के

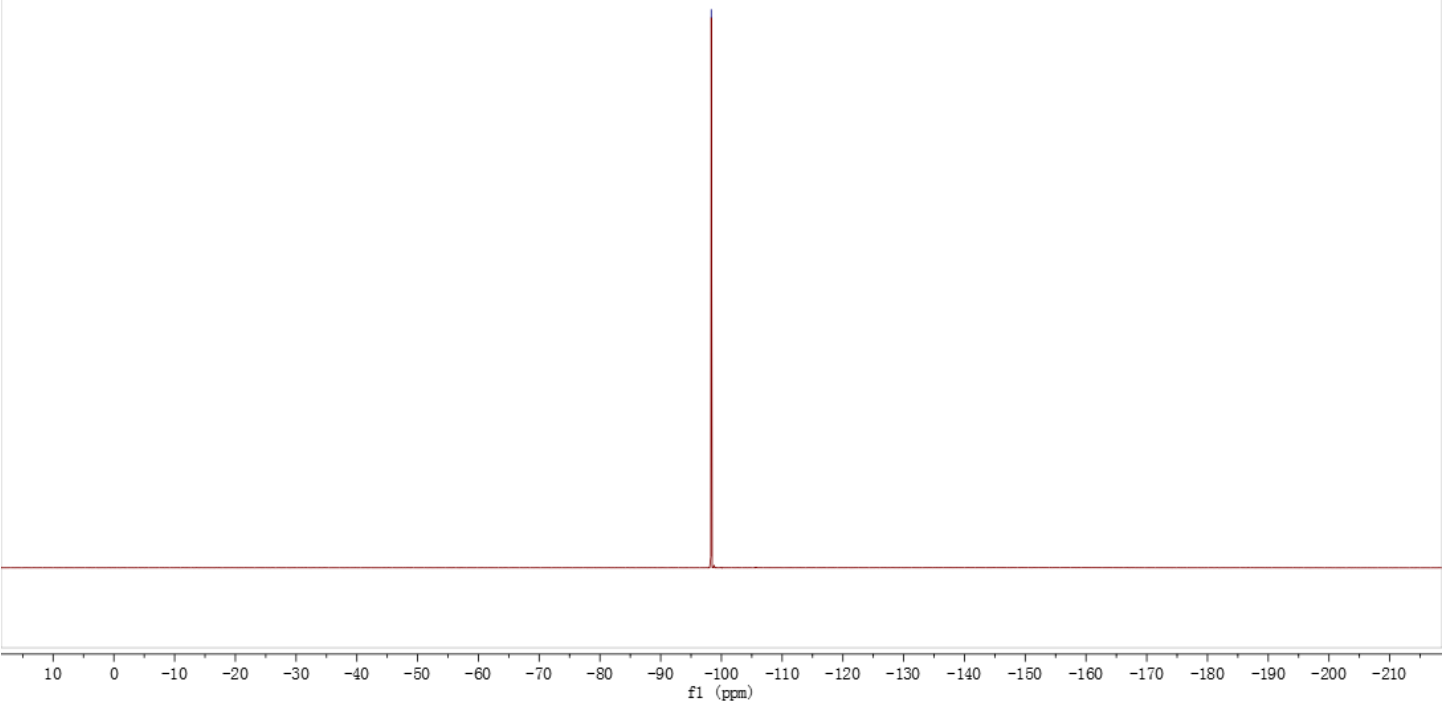




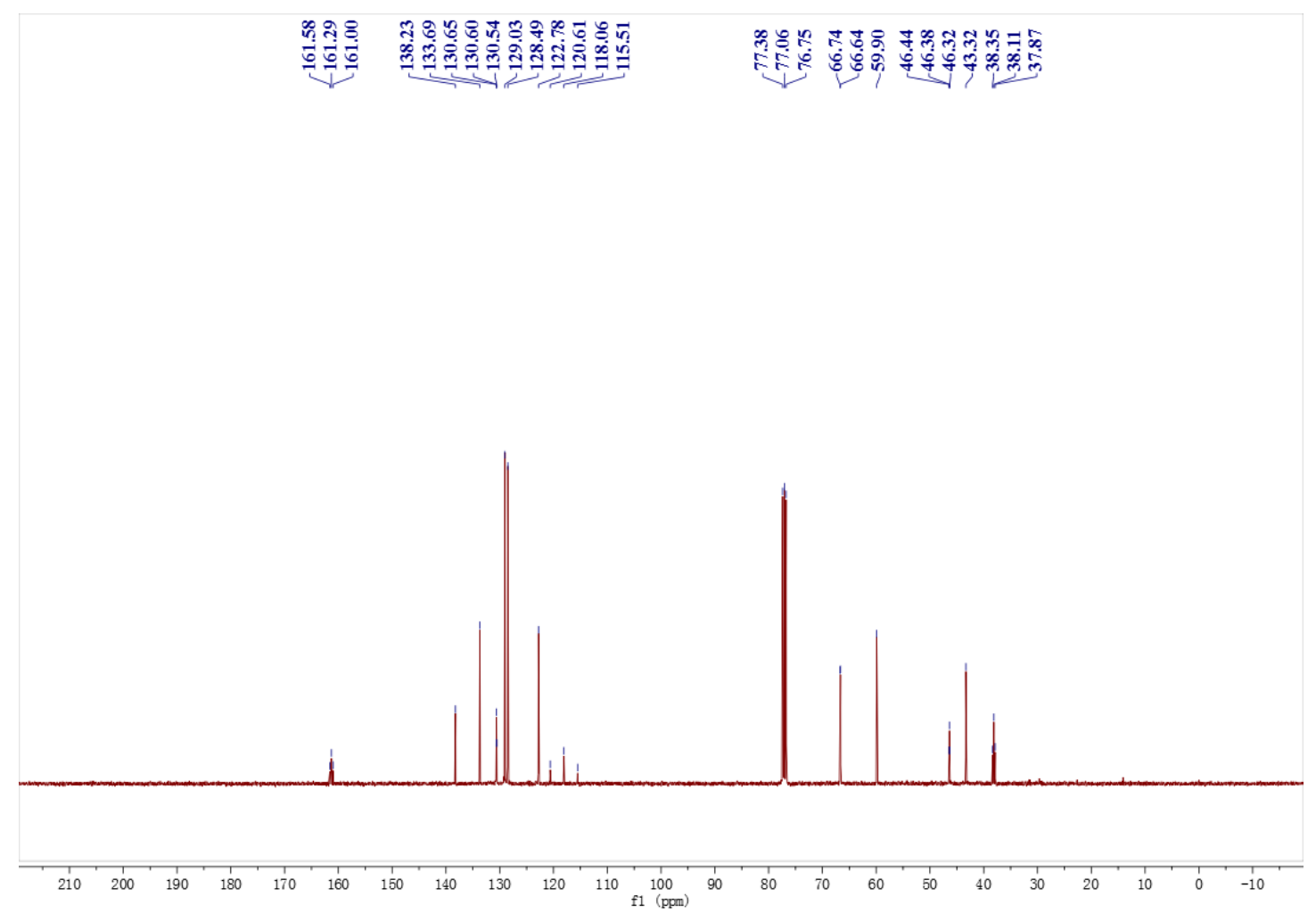


${ }^{1} \mathrm{H}$ NMR (400 MHz, CDCl $),{ }^{19} \mathrm{~F}\left\{{ }^{1} \mathrm{H}\right\}$ NMR $\left(375 \mathrm{MHz}, \mathrm{CDCl}_{3}\right),{ }^{13} \mathrm{C}\left\{{ }^{1} \mathrm{H}\right\}$ NMR (100 MHz, $\mathrm{CDCl}_{3}$ ) spectrum of product $4 \mathrm{~s}$

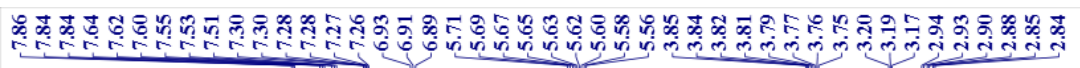
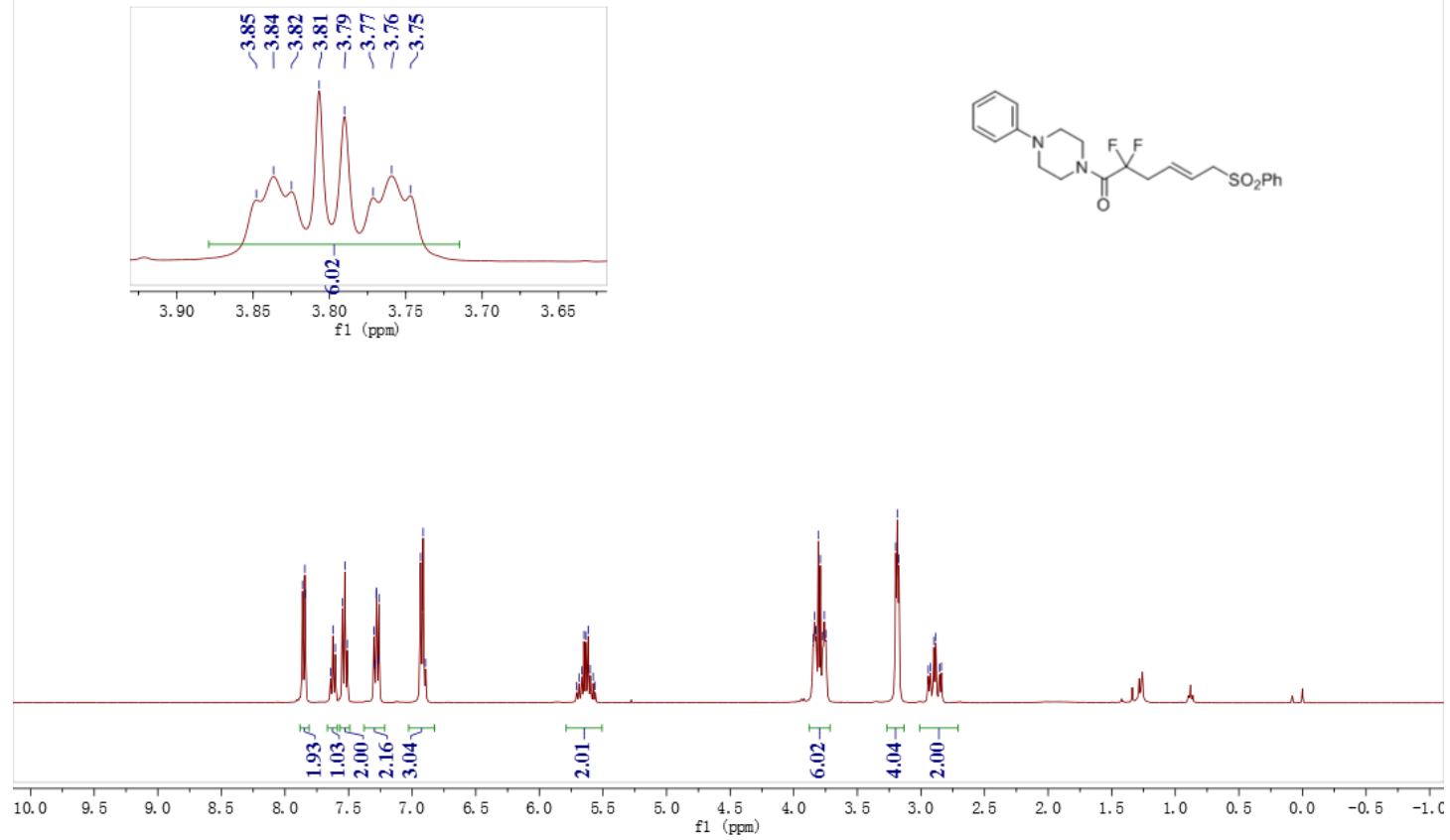

S̊ํํ

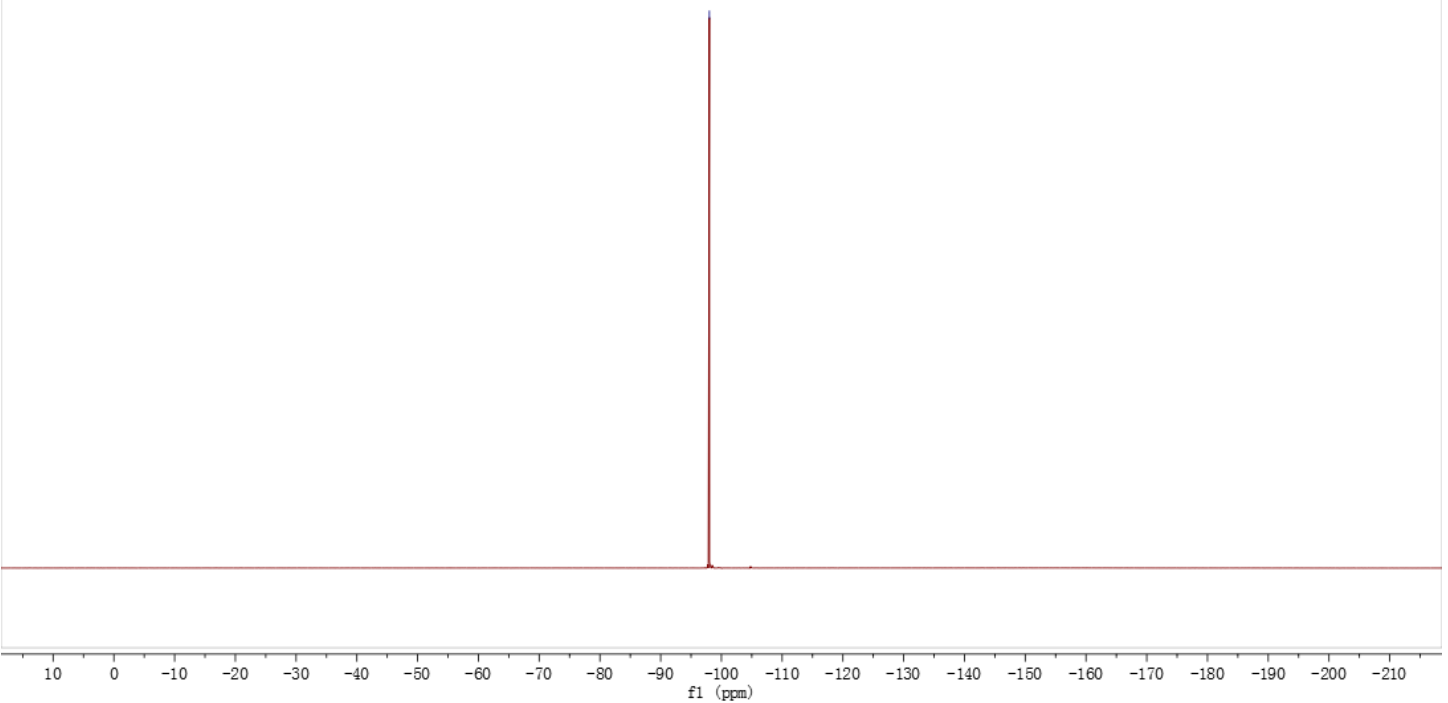




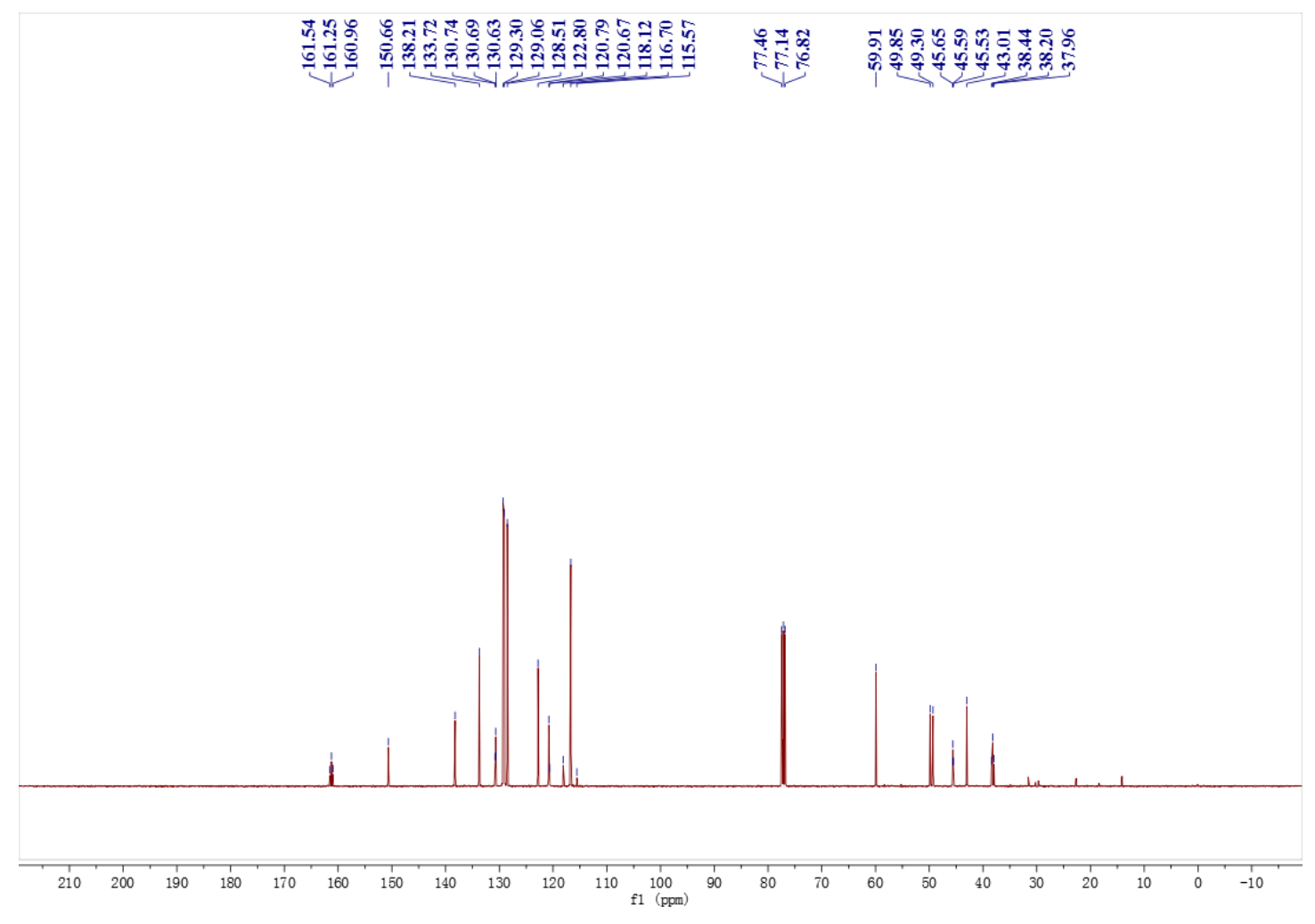


${ }^{1} \mathrm{H}$ NMR (400 MHz, $\left.\mathrm{CDCl}_{3}\right),{ }^{19} \mathrm{~F}\left\{{ }^{1} \mathrm{H}\right\}$ NMR $\left(375 \mathrm{MHz}, \mathrm{CDCl}_{3}\right),{ }^{13} \mathrm{C}\left\{{ }^{1} \mathrm{H}\right\}$ NMR (100 MHz, $\mathrm{CDCl}_{3}$ ) spectrum of product $4 \mathrm{t}$

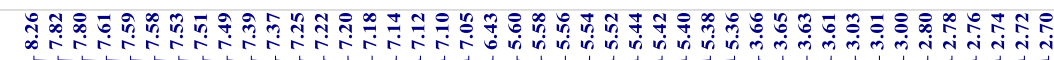
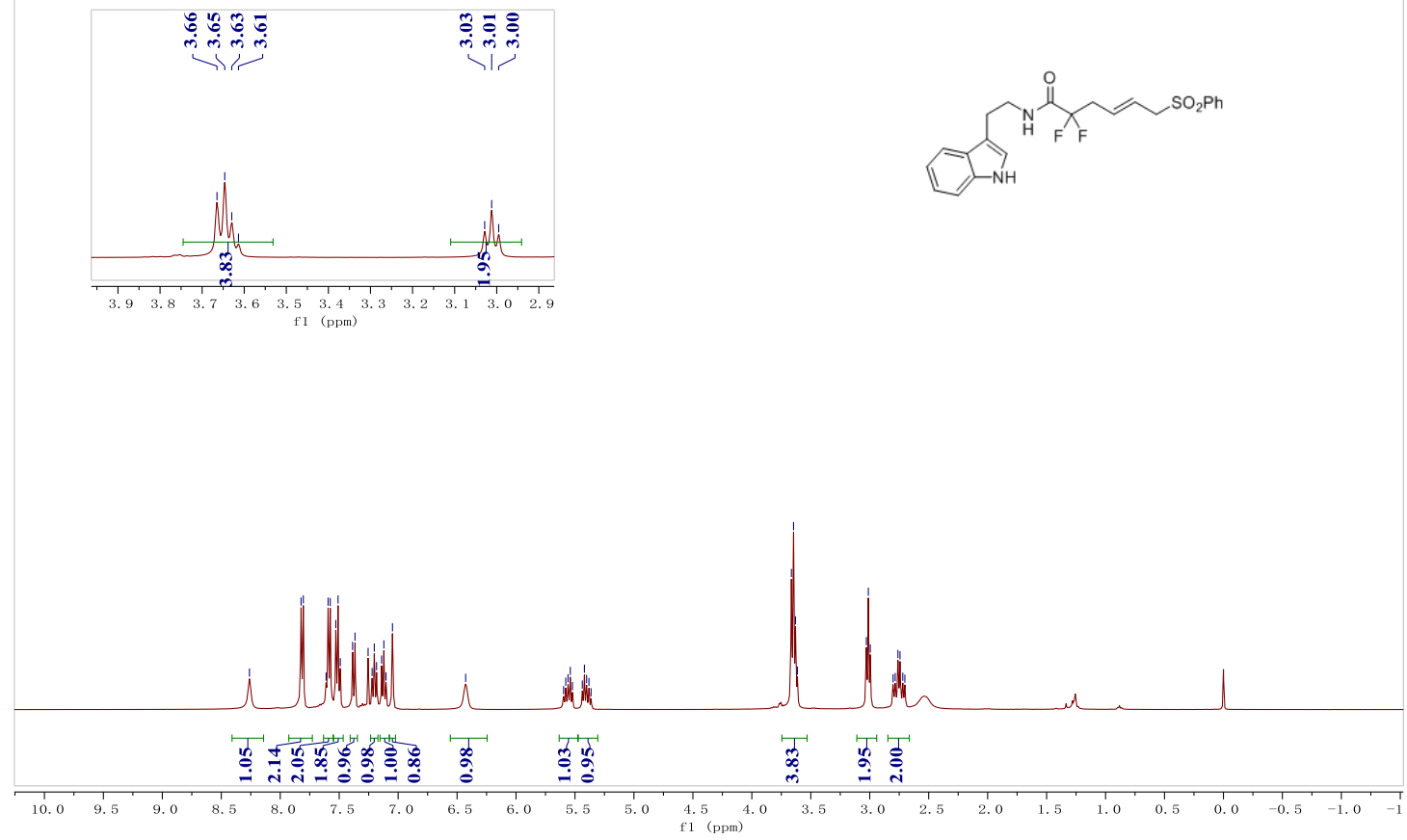

品

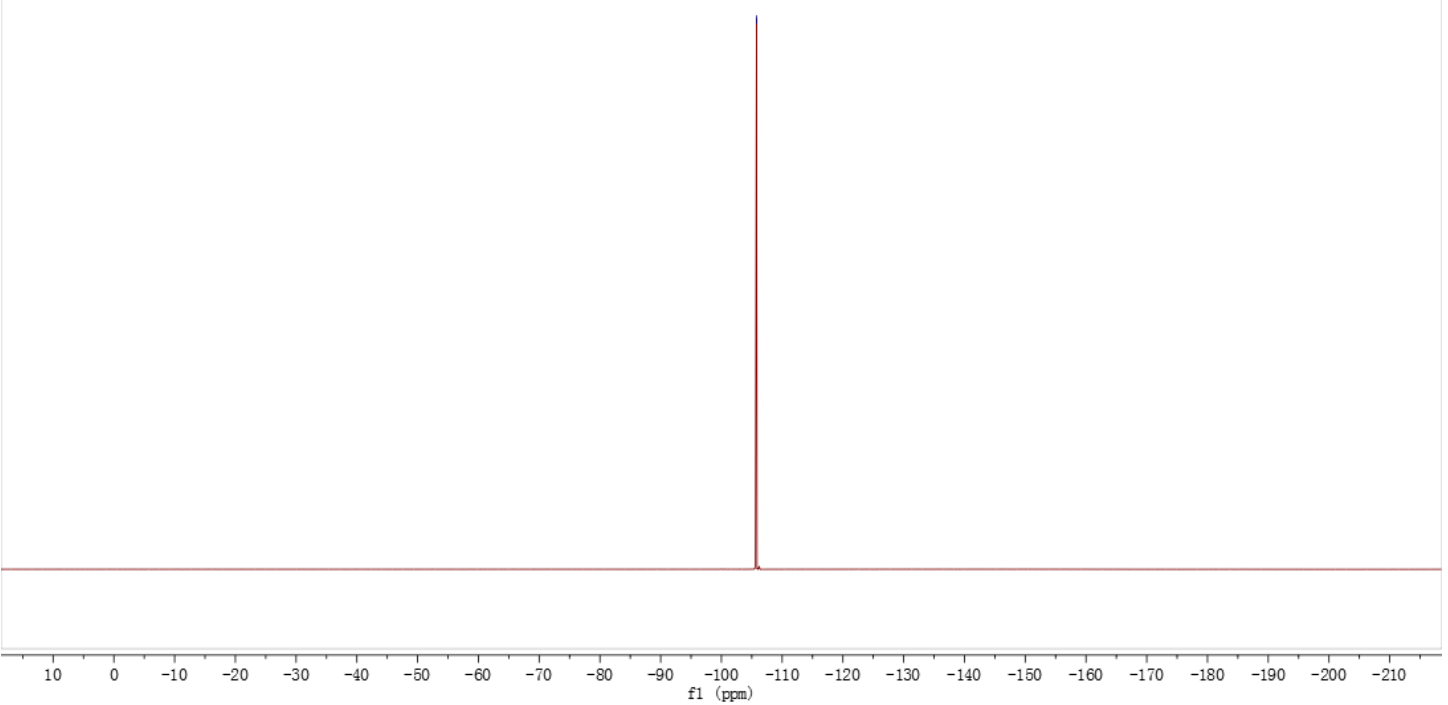




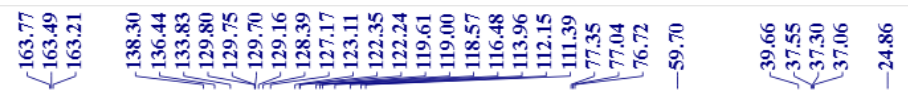

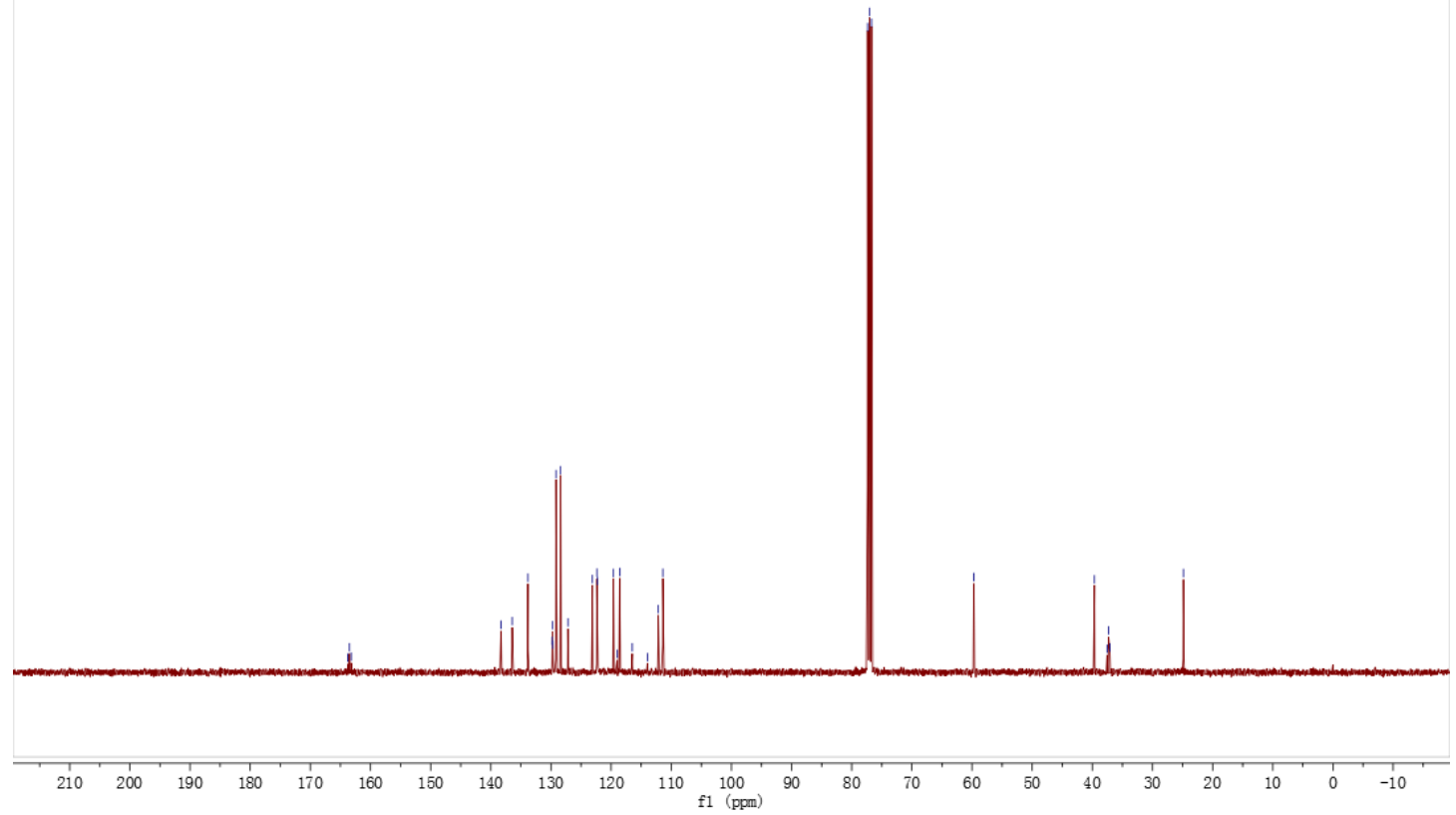


${ }^{1} \mathrm{H}$ NMR $\left(400 \mathrm{MHz}, \mathrm{CDCl}_{3}\right),{ }^{19} \mathrm{~F}\left\{{ }^{1} \mathrm{H}\right\}$ NMR $\left(375 \mathrm{MHz}, \mathrm{CDCl}_{3}\right),{ }^{13} \mathrm{C}\left\{{ }^{1} \mathrm{H}\right\}$ NMR (100 MHz, $\mathrm{CDCl}_{3}$ ) spectrum of product $5 \mathrm{~b}$

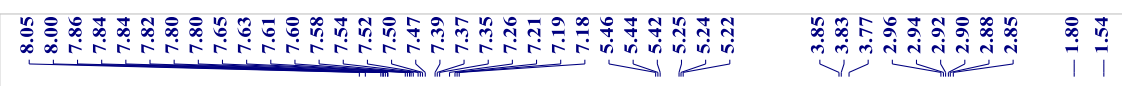

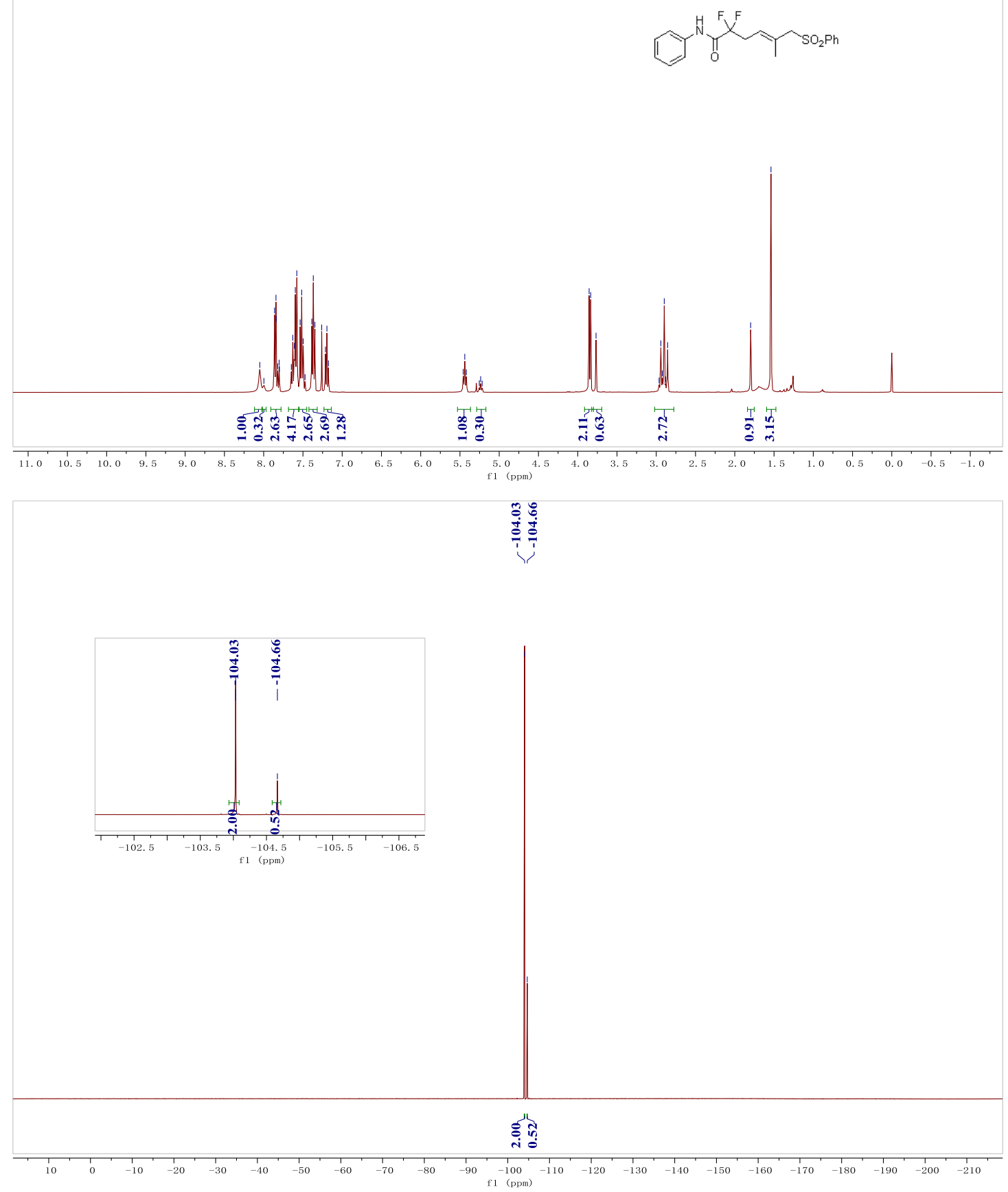




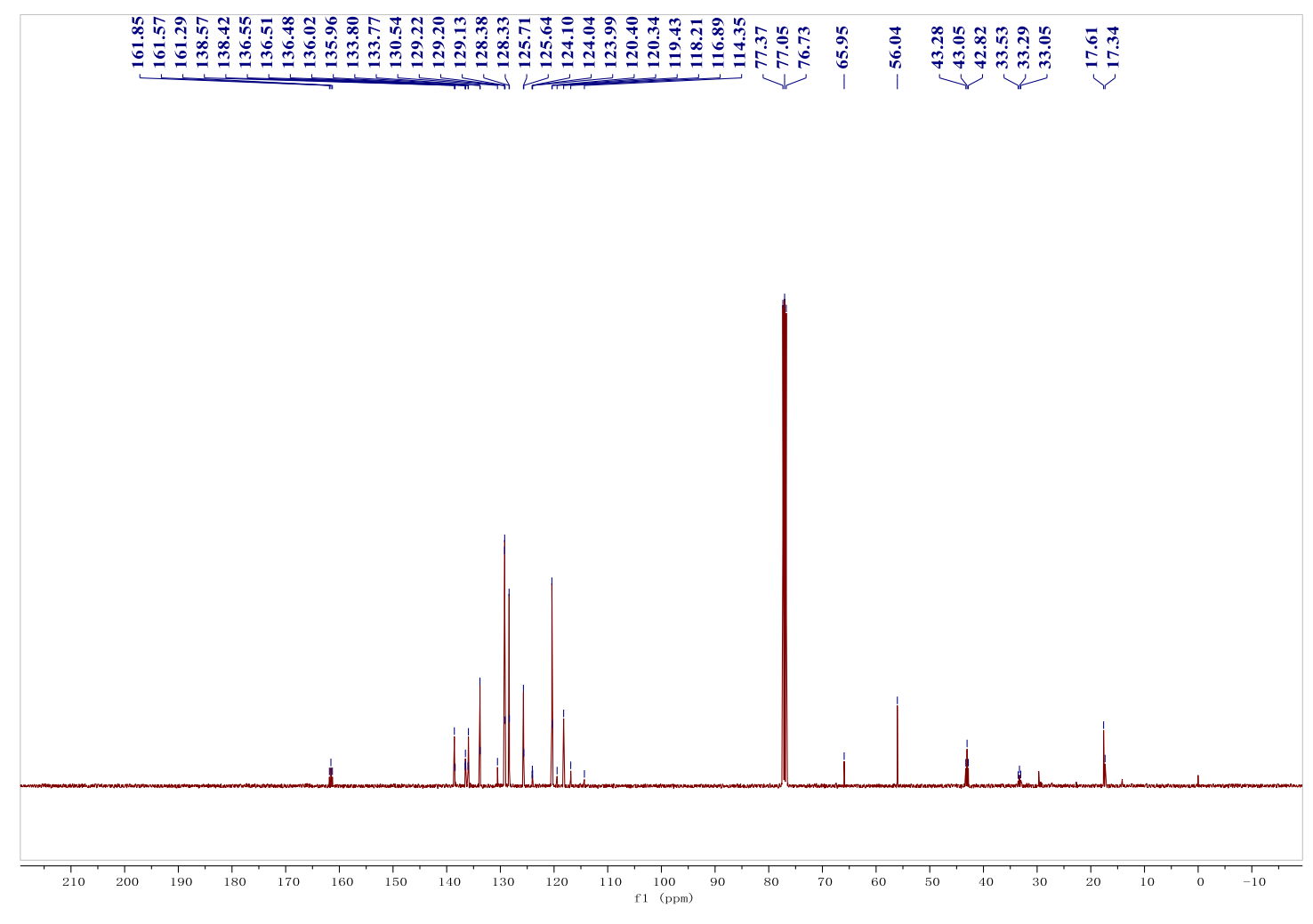


${ }^{1} \mathrm{H}$ NMR (400 MHz, CDCl $),{ }^{19} \mathrm{~F}\left\{{ }^{1} \mathrm{H}\right\}$ NMR $\left(375 \mathrm{MHz}, \mathrm{CDCl}_{3}\right),{ }^{13} \mathrm{C}\left\{{ }^{1} \mathrm{H}\right\}$ NMR (100 MHz, $\mathrm{CDCl}_{3}$ ) spectrum of product $5 \mathrm{c}$

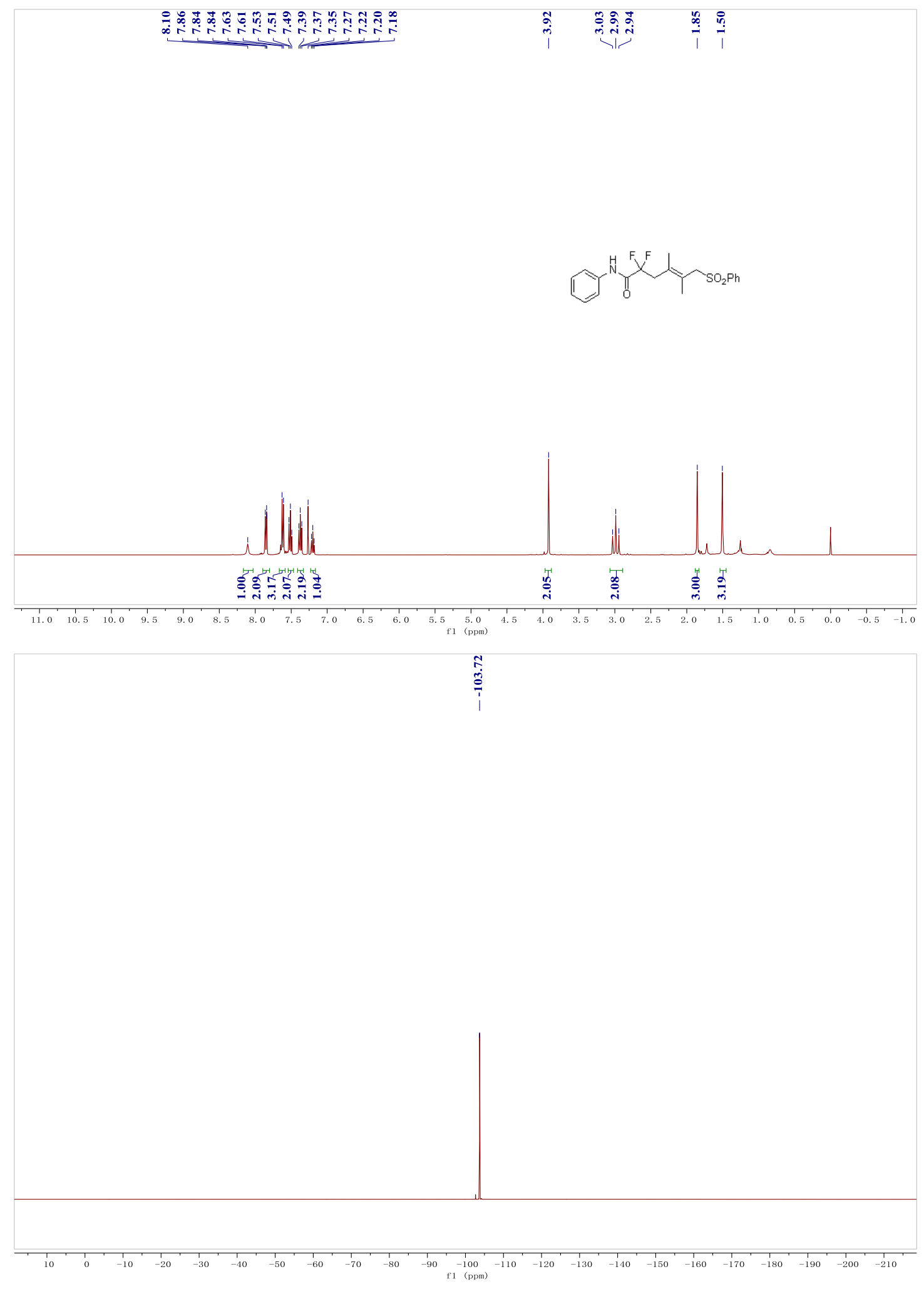




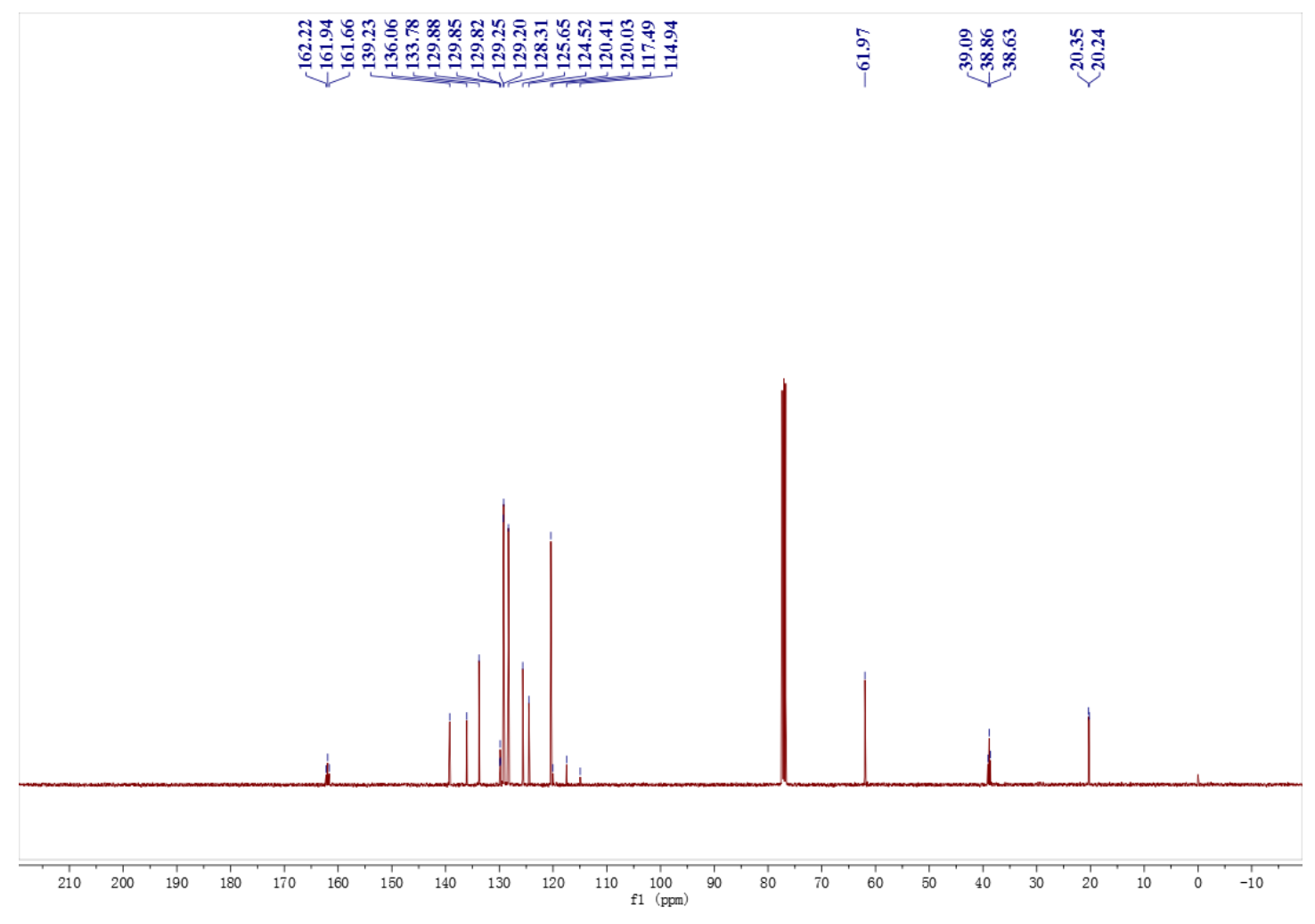


${ }^{1} \mathrm{H}$ NMR (400 MHz, CDCl $),{ }^{19} \mathrm{~F}\left\{{ }^{1} \mathrm{H}\right\}$ NMR $\left(375 \mathrm{MHz}, \mathrm{CDCl}_{3}\right),{ }^{13} \mathrm{C}\left\{{ }^{1} \mathrm{H}\right\}$ NMR (100 MHz, $\mathrm{CDCl}_{3}$ ) spectrum of product $5 \mathrm{~d}$

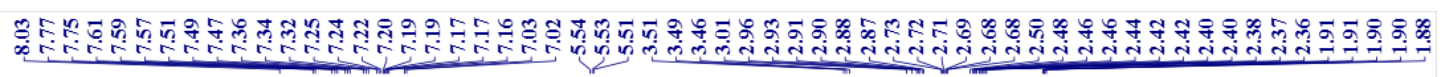
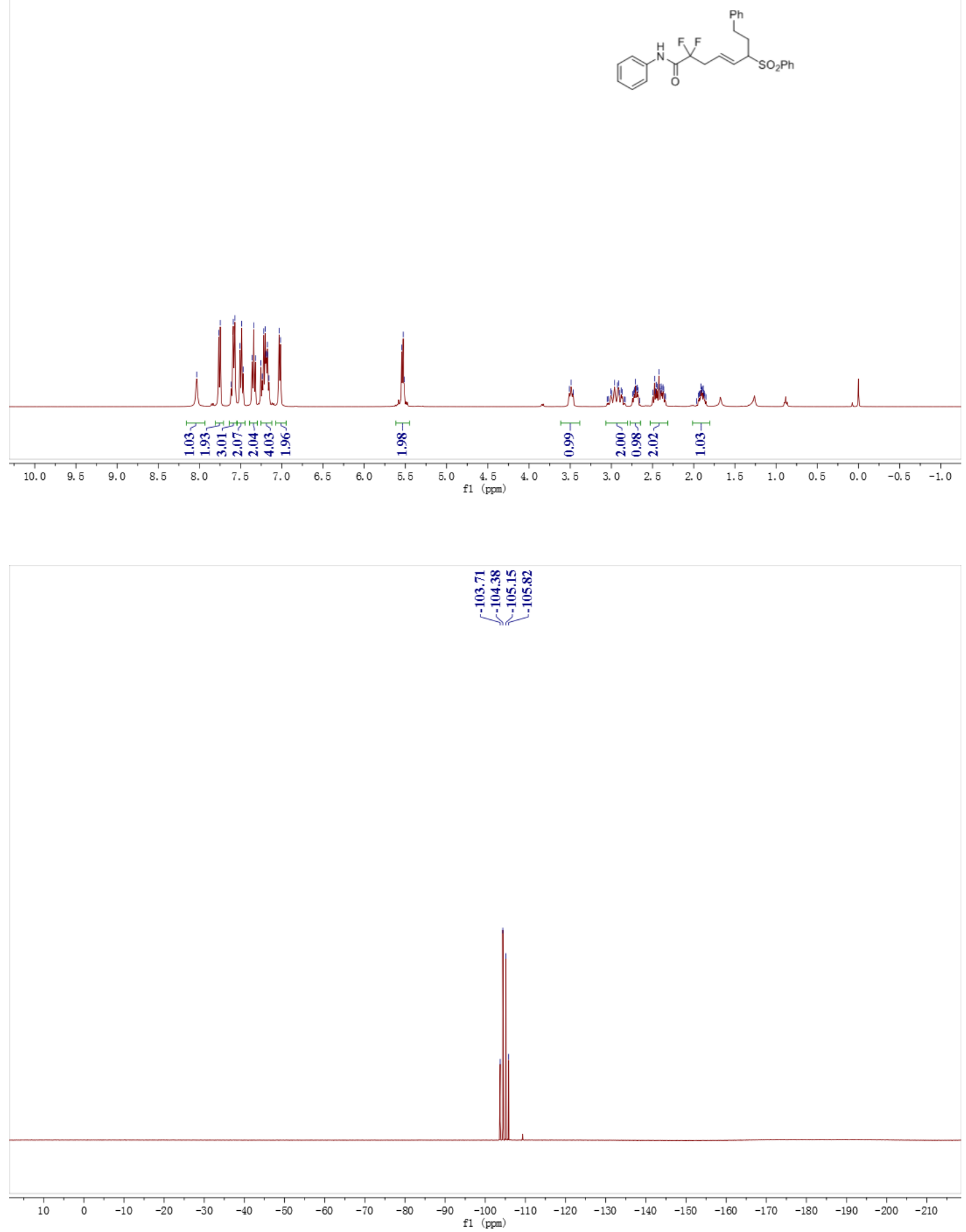


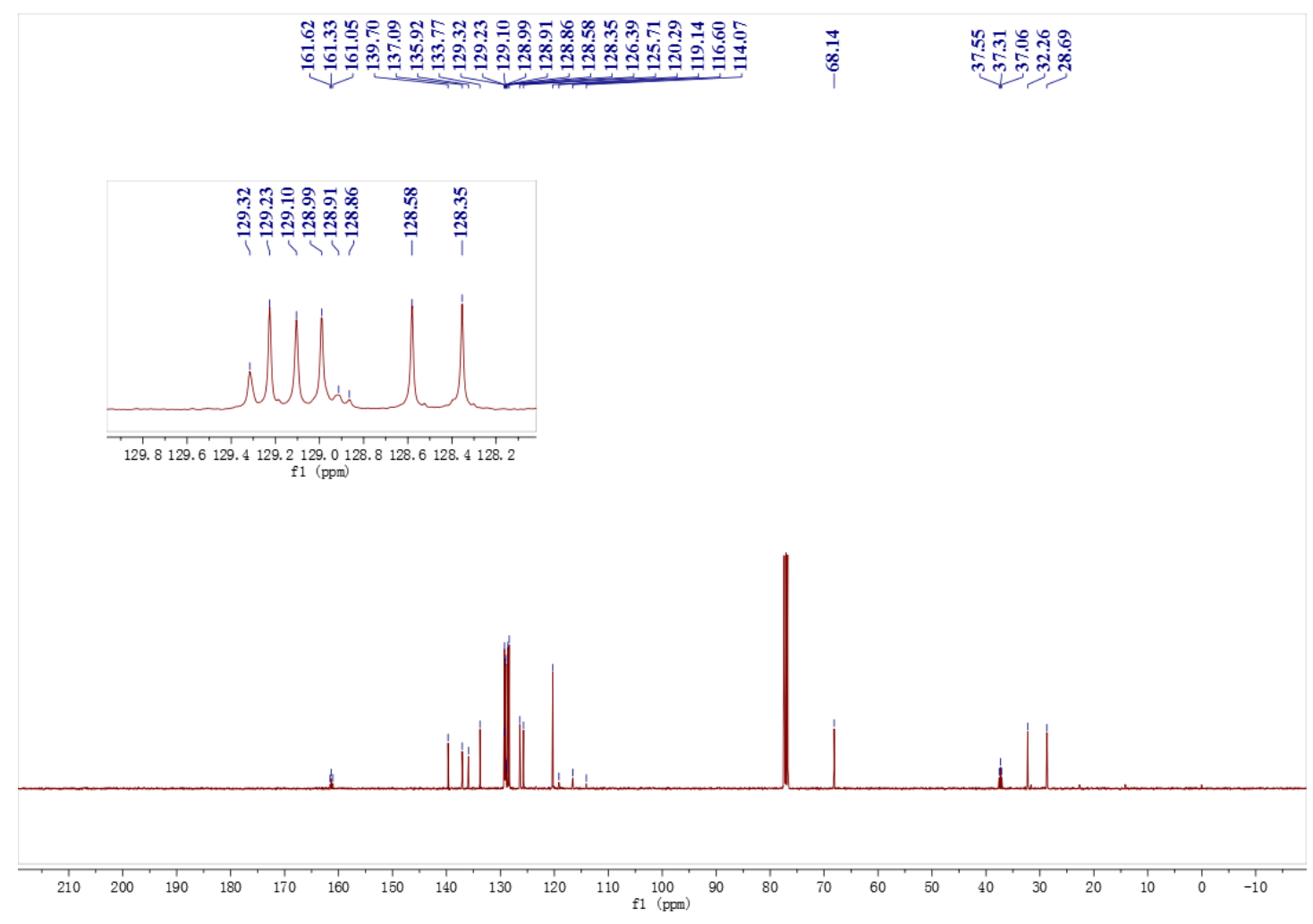


${ }^{1} \mathrm{H}$ NMR (400 MHz, CDCl $),{ }^{19} \mathrm{~F}\left\{{ }^{1} \mathrm{H}\right\}$ NMR $\left(375 \mathrm{MHz}, \mathrm{CDCl}_{3}\right),{ }^{13} \mathrm{C}\left\{{ }^{1} \mathrm{H}\right\}$ NMR (100 MHz, $\mathrm{CDCl}_{3}$ ) spectrum of product $5 \mathrm{e}$

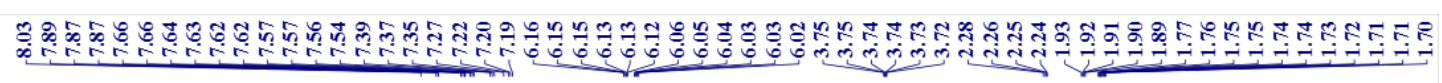
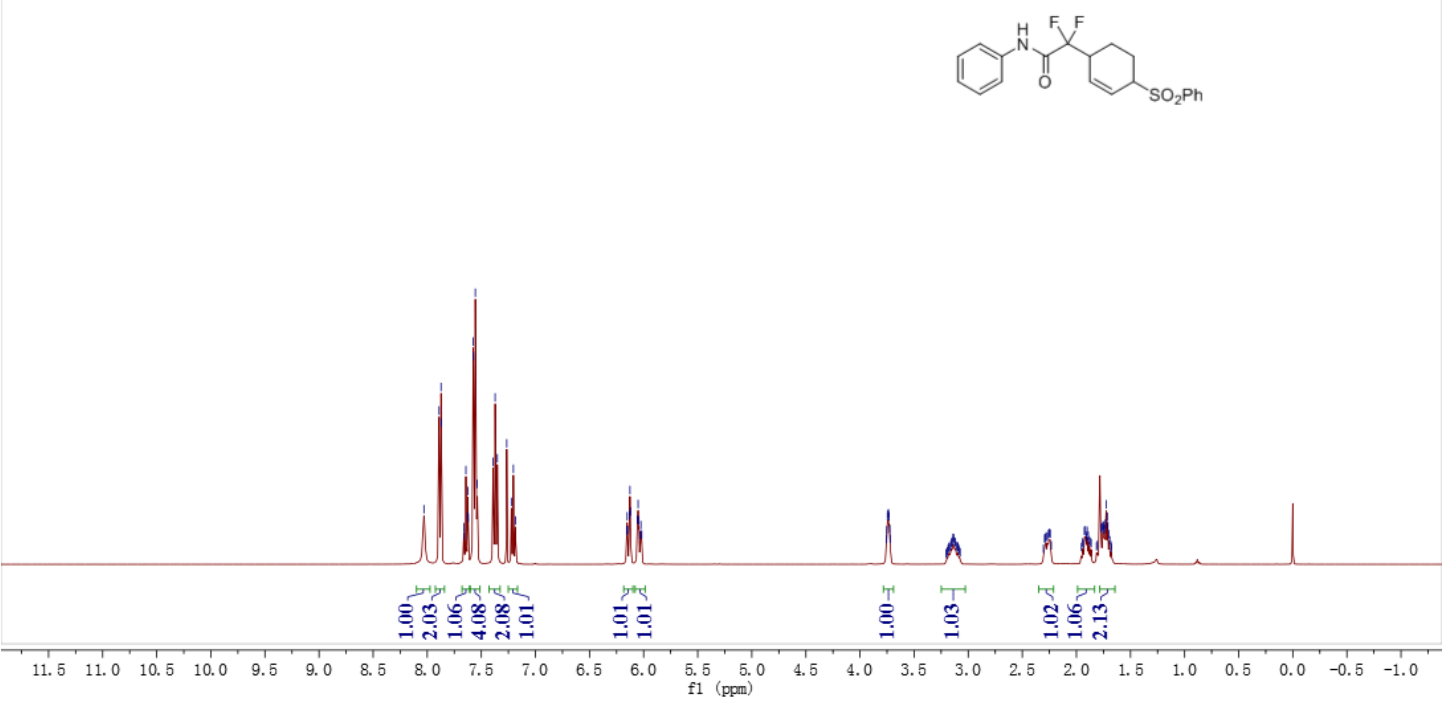

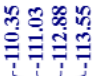

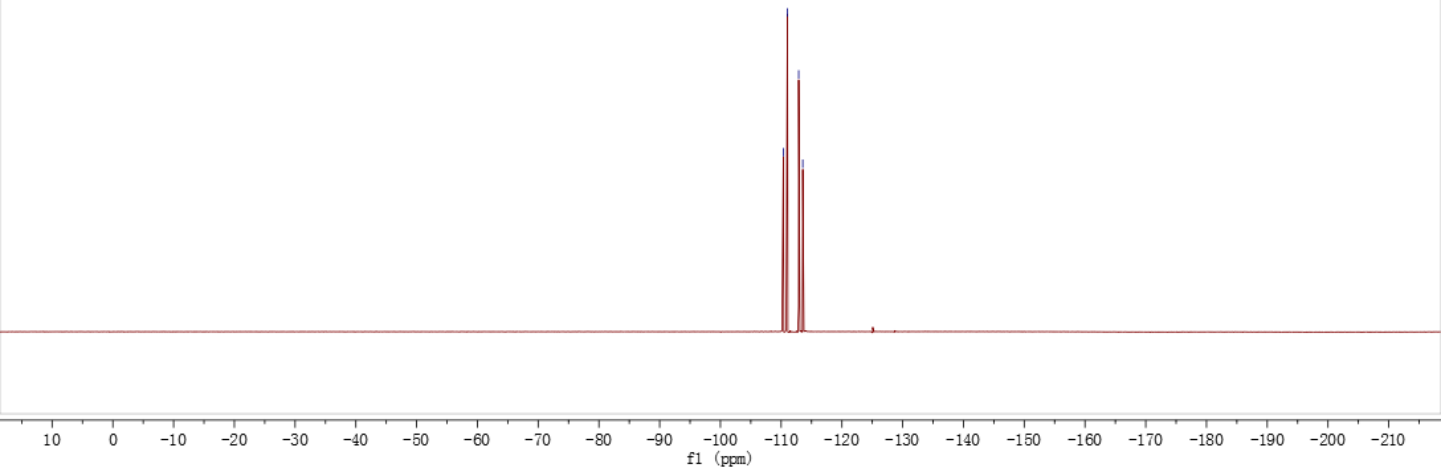




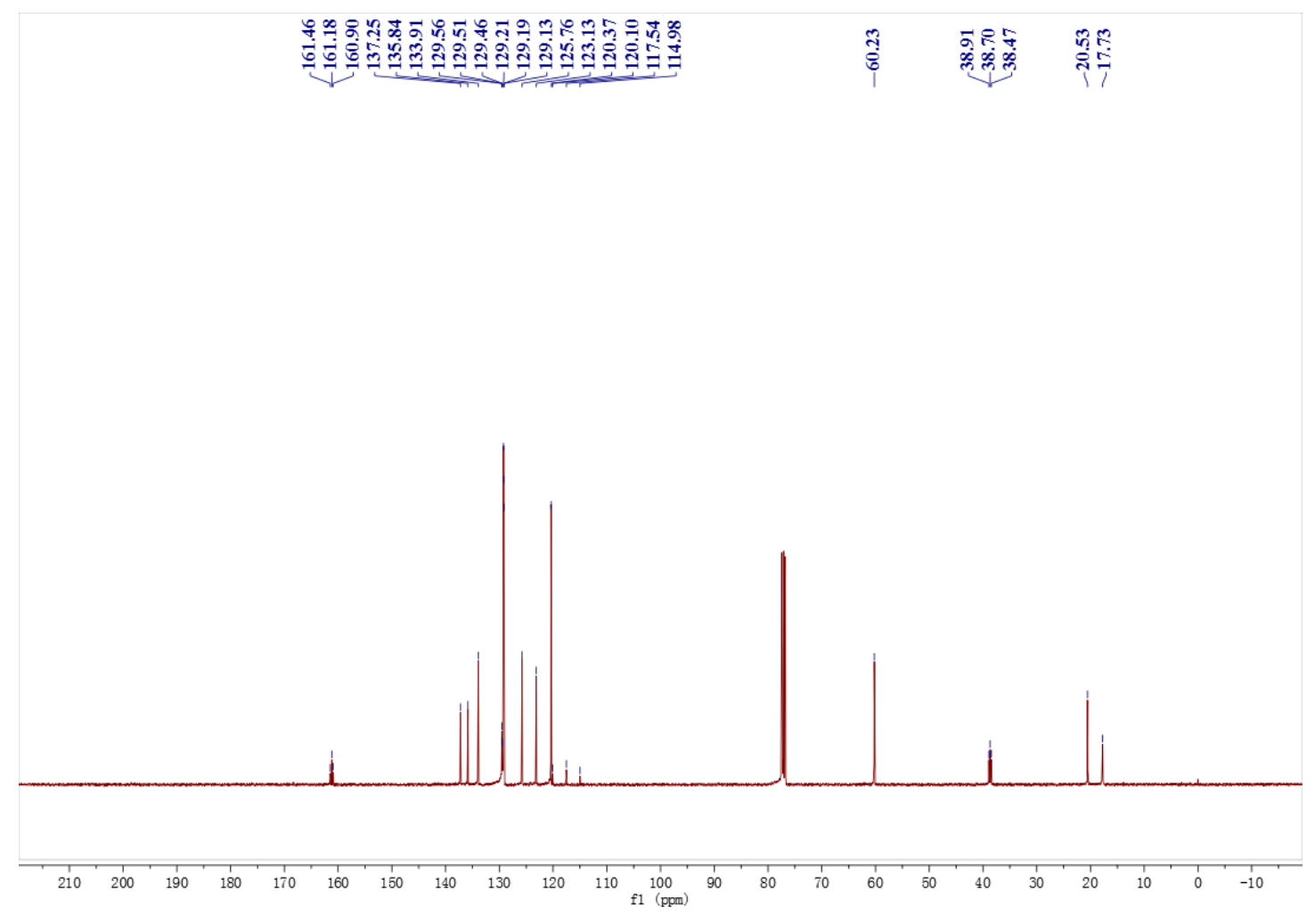


${ }^{1} \mathrm{H}$ NMR (400 MHz, CDCl $),{ }^{19} \mathrm{~F}\left\{{ }^{1} \mathrm{H}\right\}$ NMR $\left(375 \mathrm{MHz}, \mathrm{CDCl}_{3}\right),{ }^{13} \mathrm{C}\left\{{ }^{1} \mathrm{H}\right\}$ NMR (100 MHz, $\mathrm{CDCl}_{3}$ ) spectrum of product $5 \mathrm{f}$

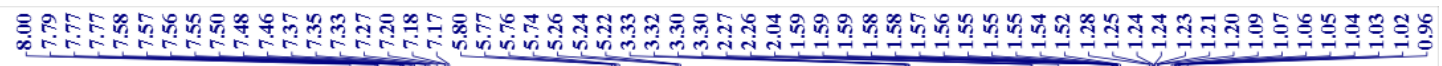

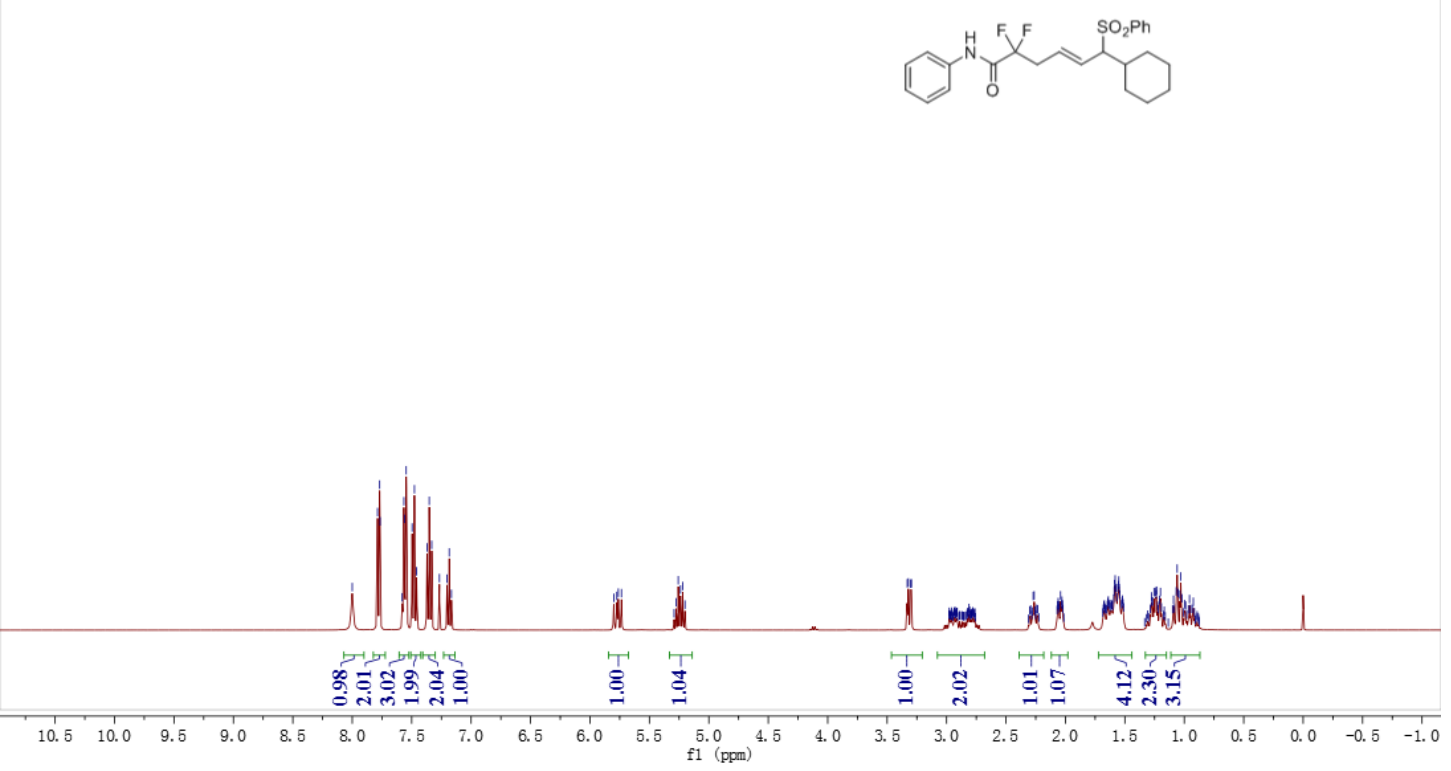

क⿻日乚

S인

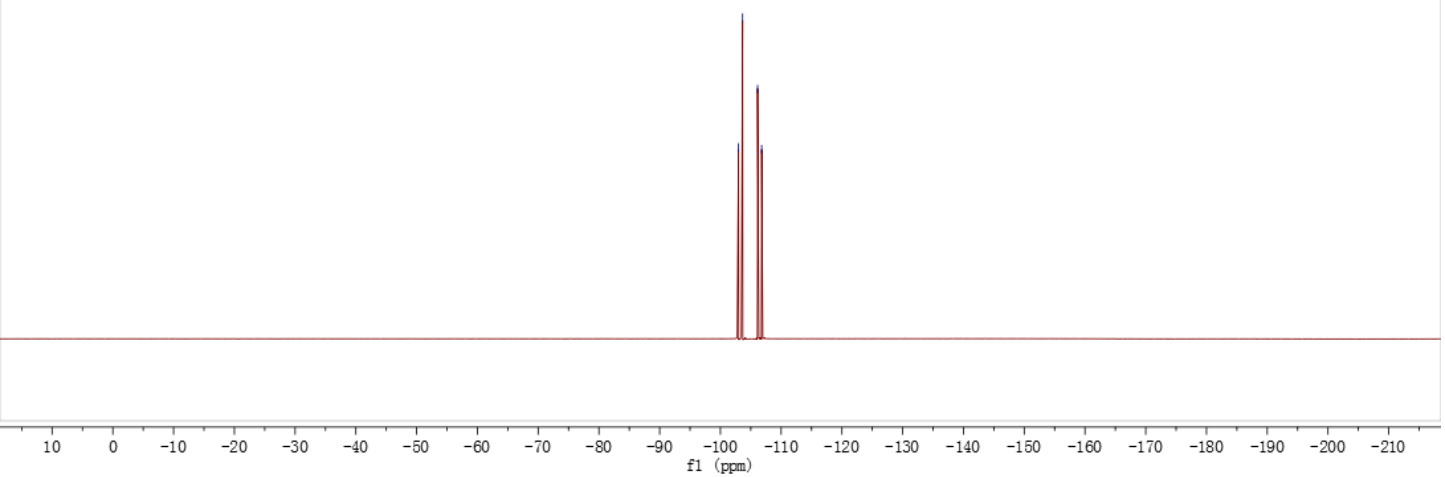




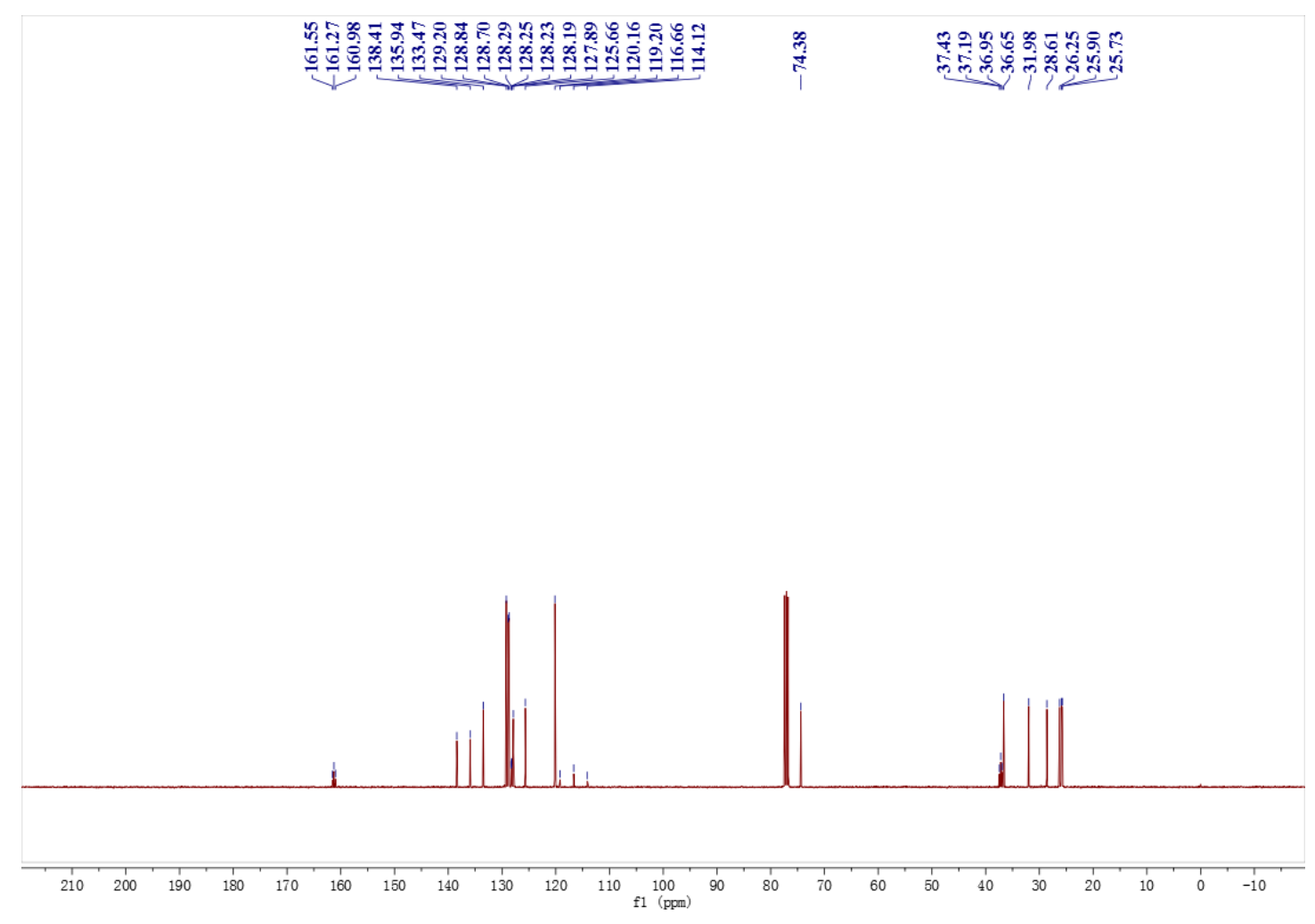


${ }^{1} \mathrm{H}$ NMR (400 MHz, DMSO- $\left.d_{6}\right),{ }^{19} \mathrm{~F}\left\{{ }^{1} \mathrm{H}\right\}$ NMR $\left(375 \mathrm{MHz}\right.$, DMSO- $\left.d_{6}\right),{ }^{13} \mathrm{C}\left\{{ }^{1} \mathrm{H}\right\}$ NMR (100 MHz, DMSO- $d_{6}$ ) spectrum of product $6 \mathrm{~b}$

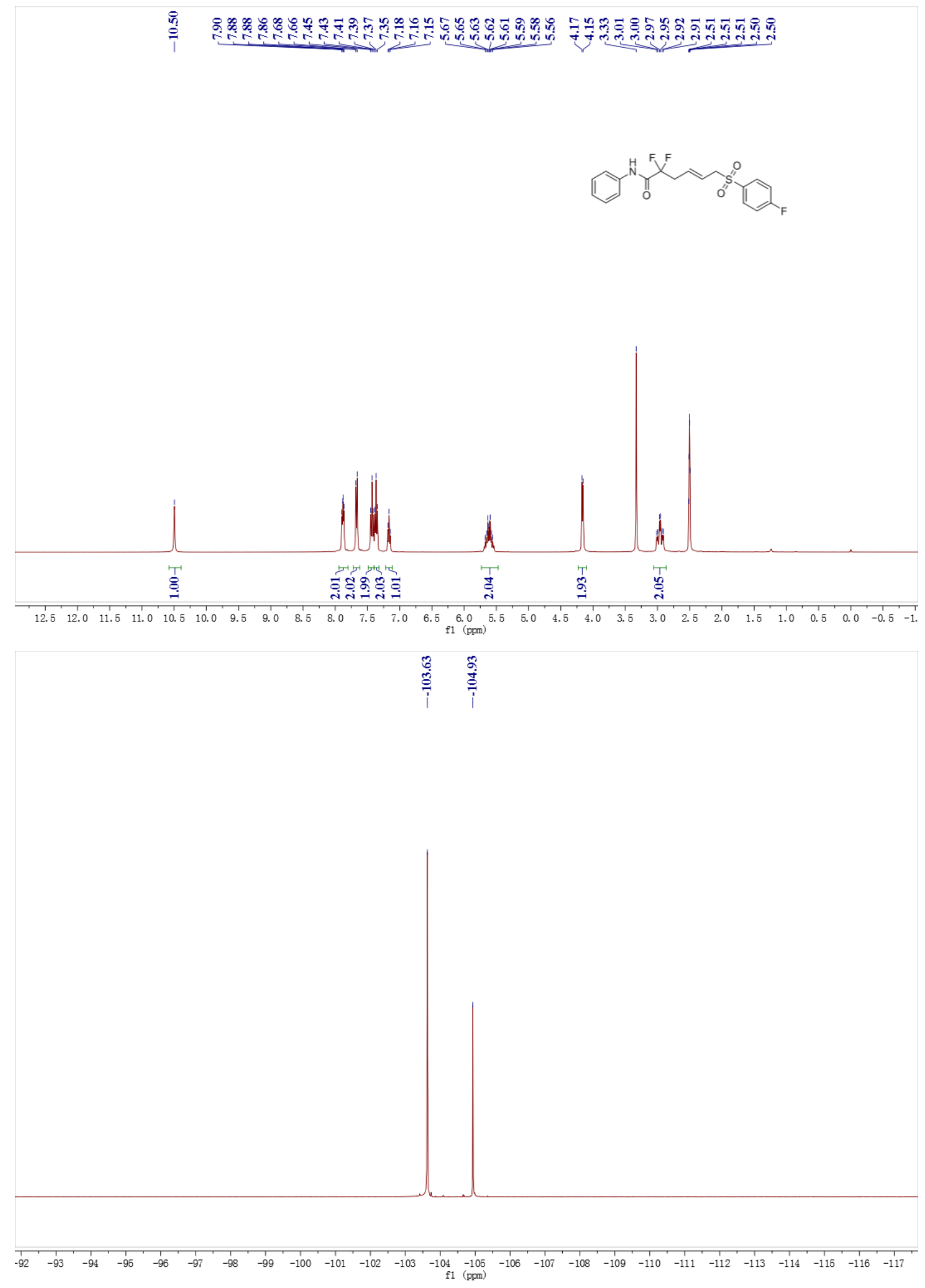




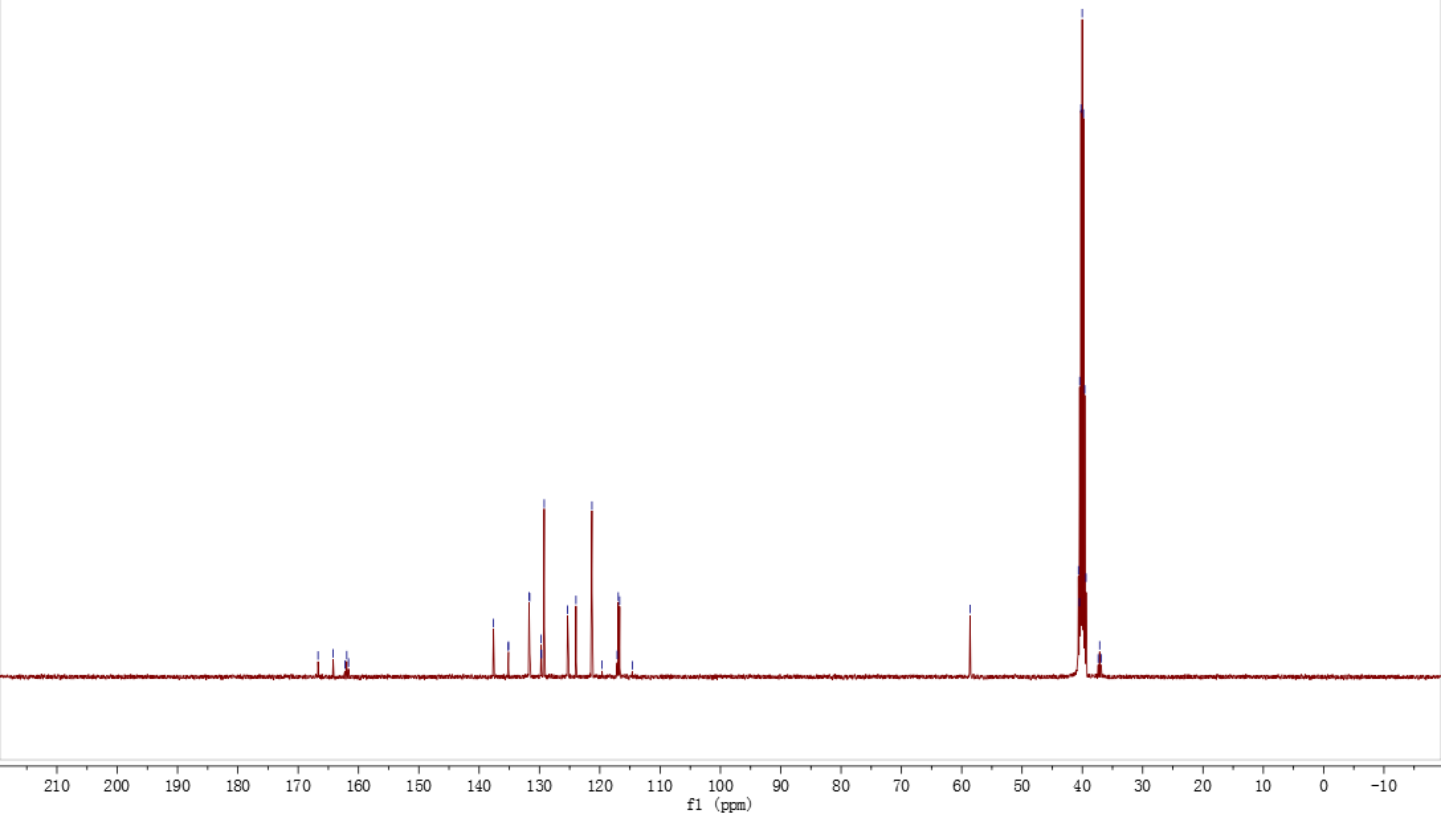


${ }^{1} \mathrm{H}$ NMR (400 MHz, DMSO- $\left.d_{6}\right),{ }^{19} \mathrm{~F}\left\{{ }^{1} \mathrm{H}\right\}$ NMR $\left(375 \mathrm{MHz}\right.$, DMSO- $\left.d_{6}\right),{ }^{13} \mathrm{C}\left\{{ }^{1} \mathrm{H}\right\}$ NMR (100 MHz, DMSO- $d_{6}$ ) spectrum of product $6 \mathrm{c}$

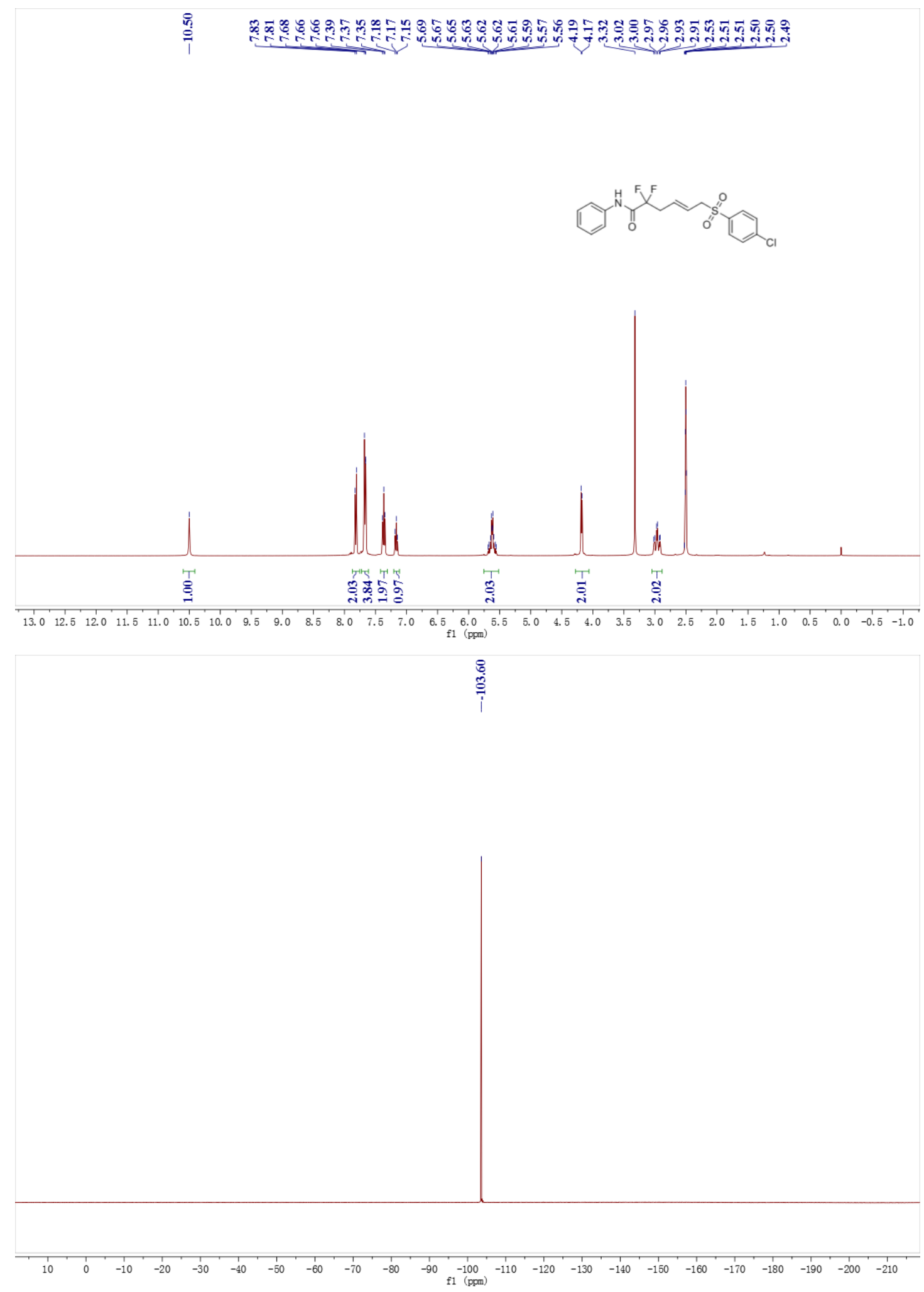




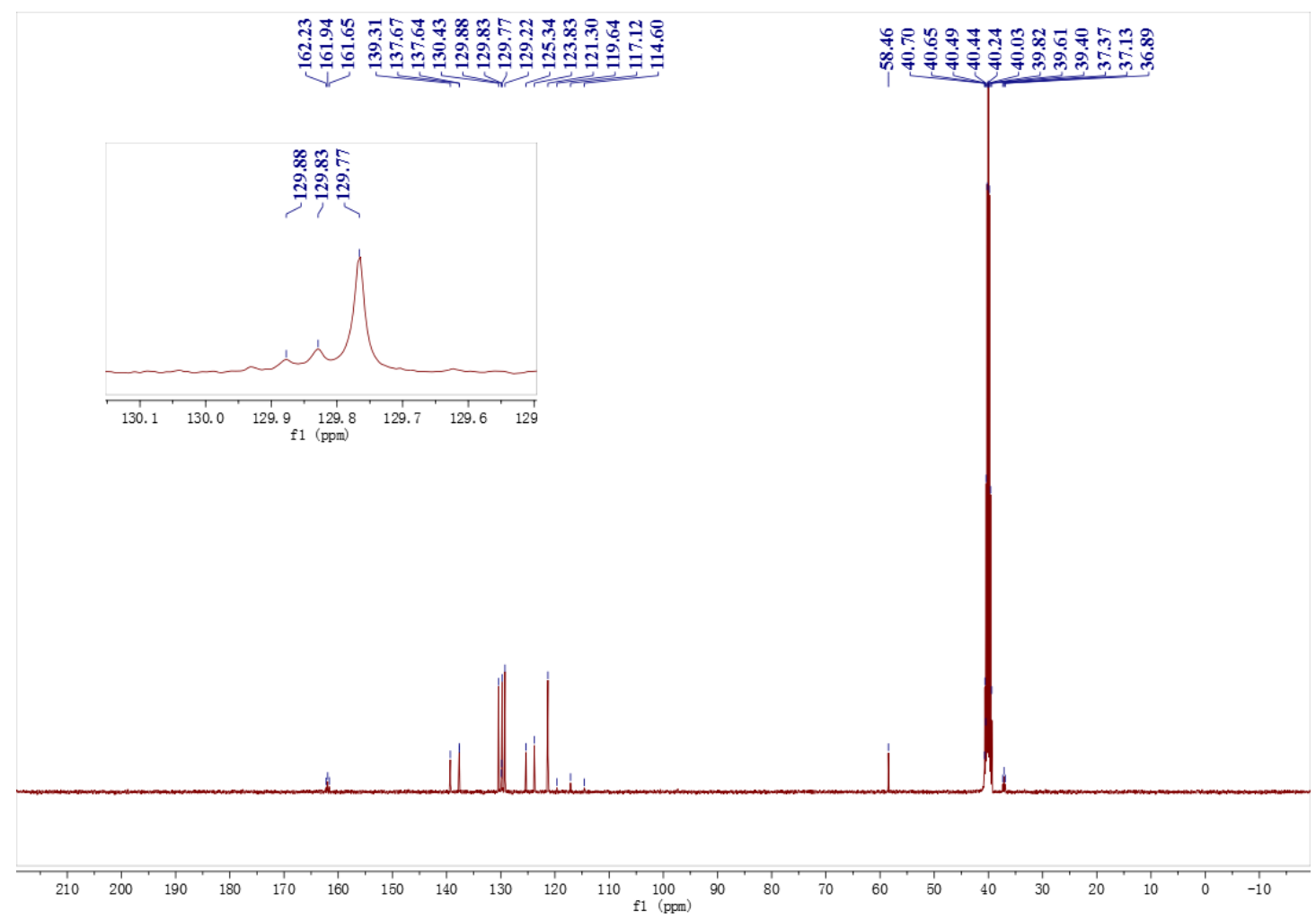


${ }^{1} \mathrm{H}$ NMR (400 MHz, DMSO-d $\left.d_{6}\right),{ }^{19} \mathrm{~F}\left\{{ }^{1} \mathrm{H}\right\}$ NMR (375 MHz, DMSO- $\left.d_{6}\right),{ }^{13} \mathrm{C}\left\{{ }^{1} \mathrm{H}\right\}$ NMR (100 MHz, DMSO- $d_{6}$ ) spectrum of product $6 \mathrm{~d}$

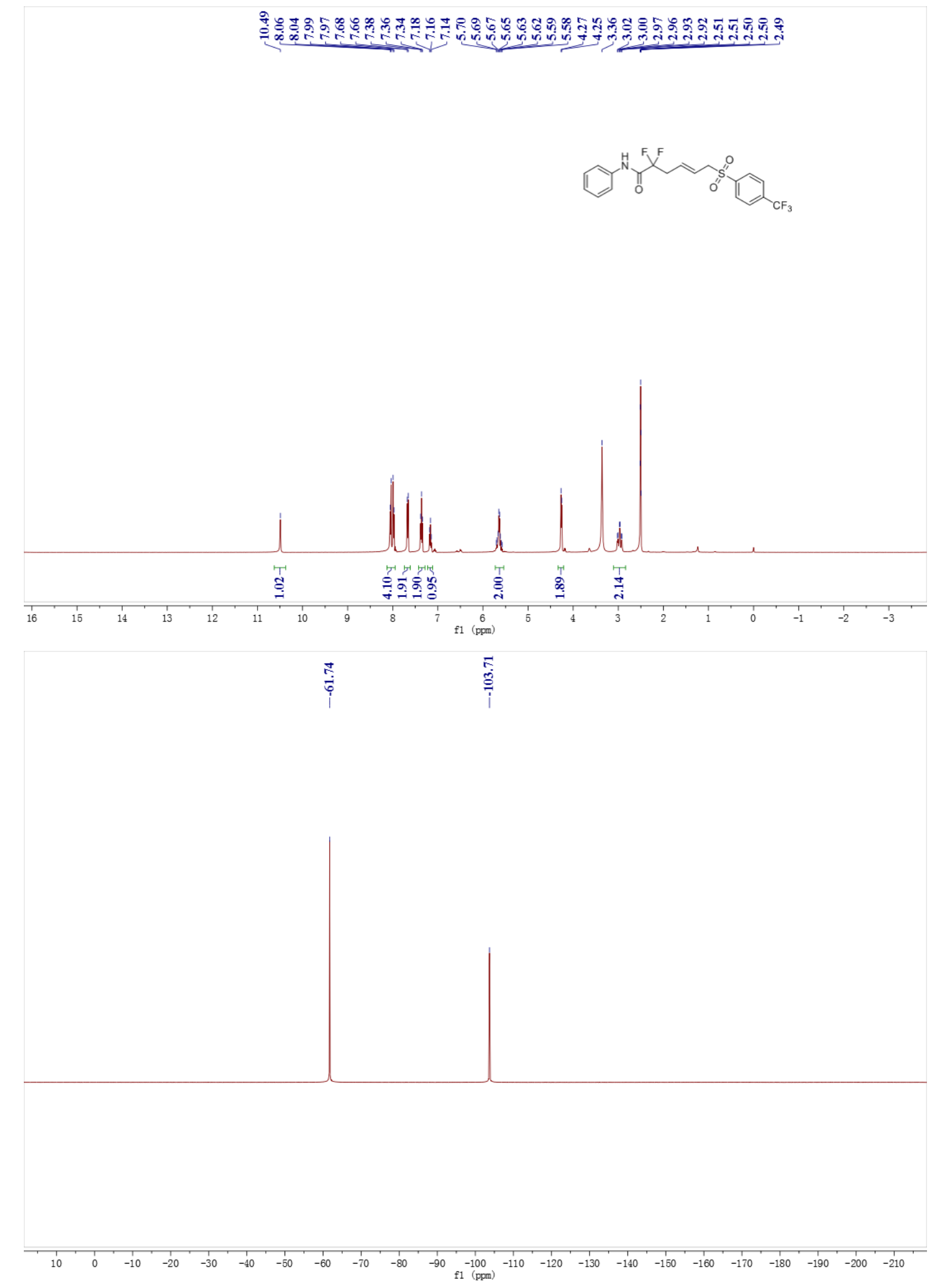




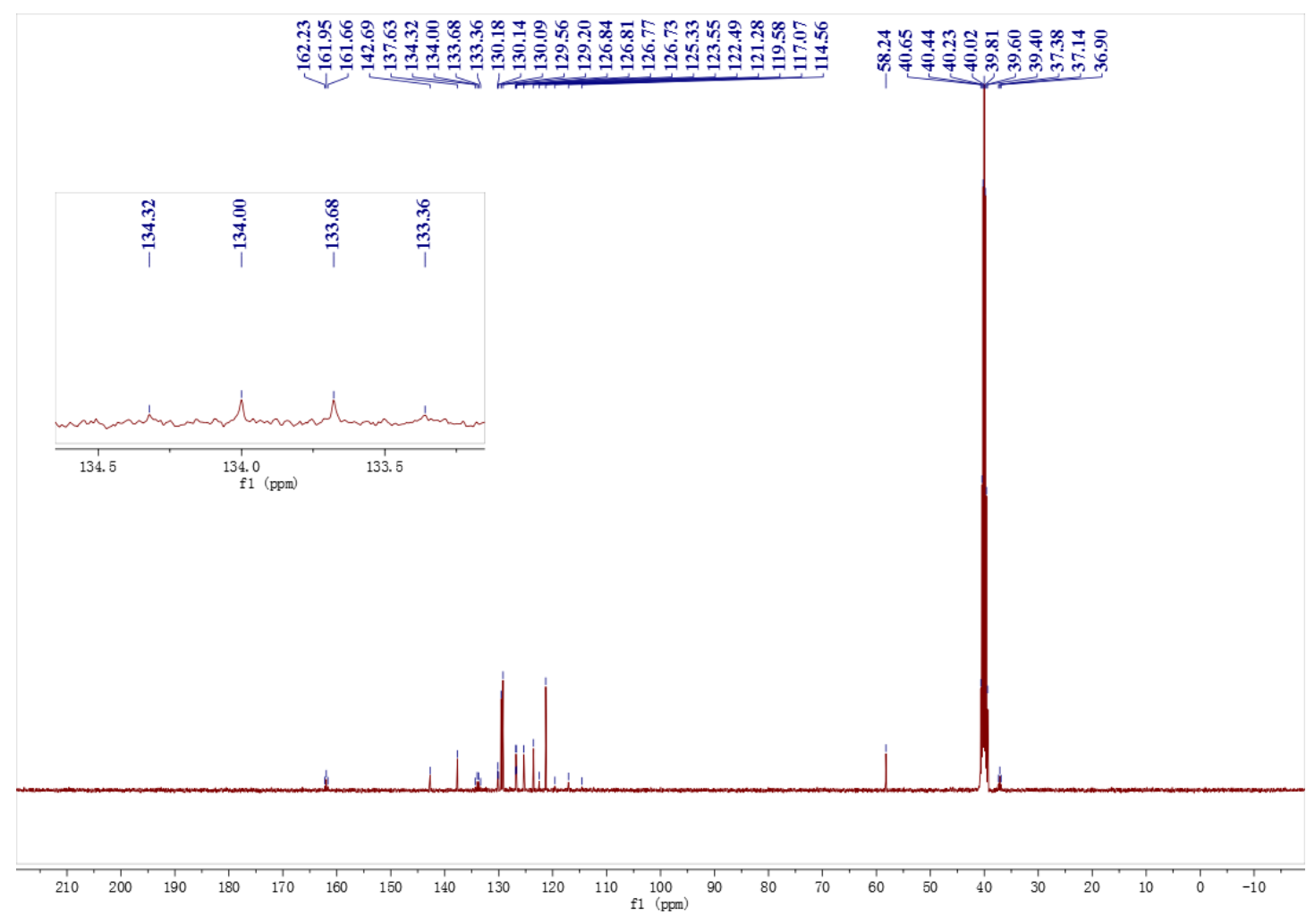


${ }^{1} \mathrm{H}$ NMR (400 MHz, CDCl $),{ }^{19} \mathrm{~F}\left\{{ }^{1} \mathrm{H}\right\}$ NMR $\left(375 \mathrm{MHz}, \mathrm{CDCl}_{3}\right),{ }^{13} \mathrm{C}\left\{{ }^{1} \mathrm{H}\right\}$ NMR (100 MHz, $\mathrm{CDCl}_{3}$ ) spectrum of product $6 \mathrm{e}$

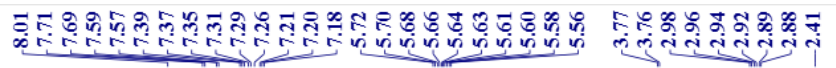
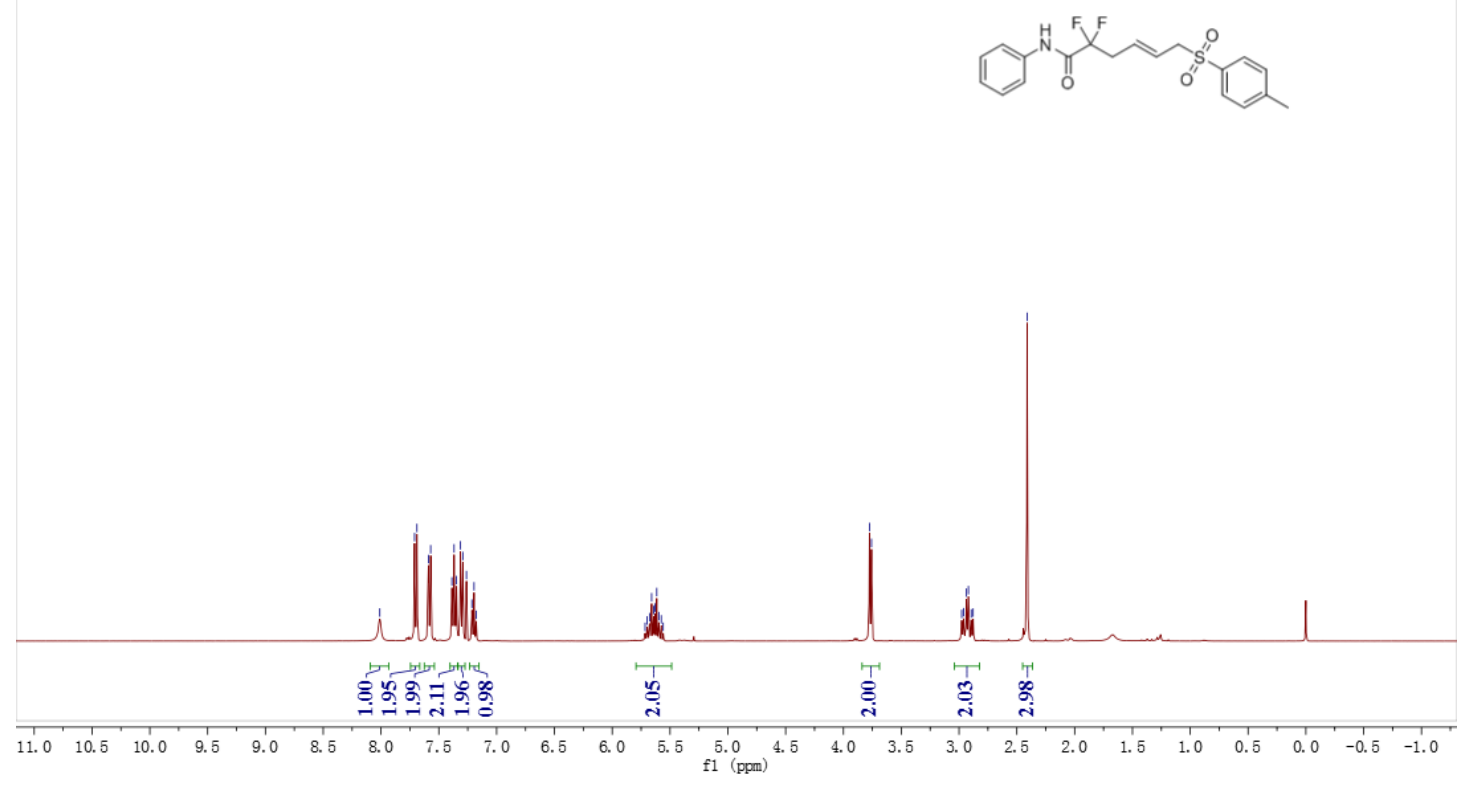

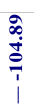

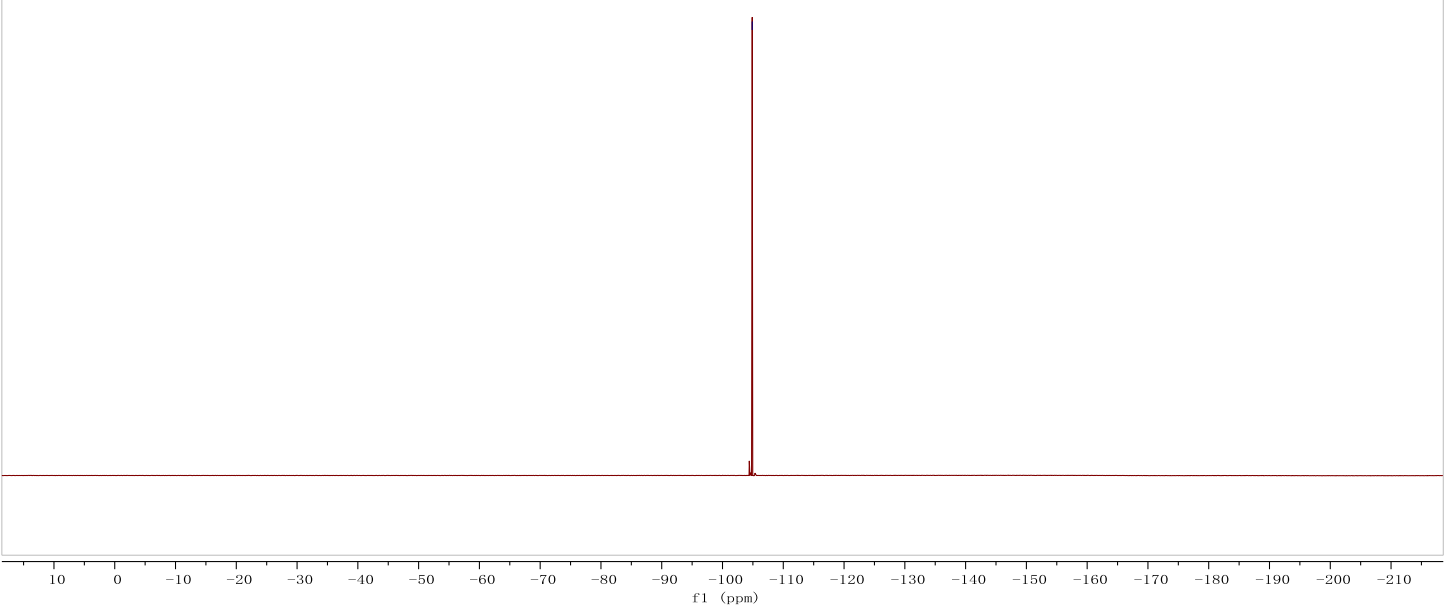




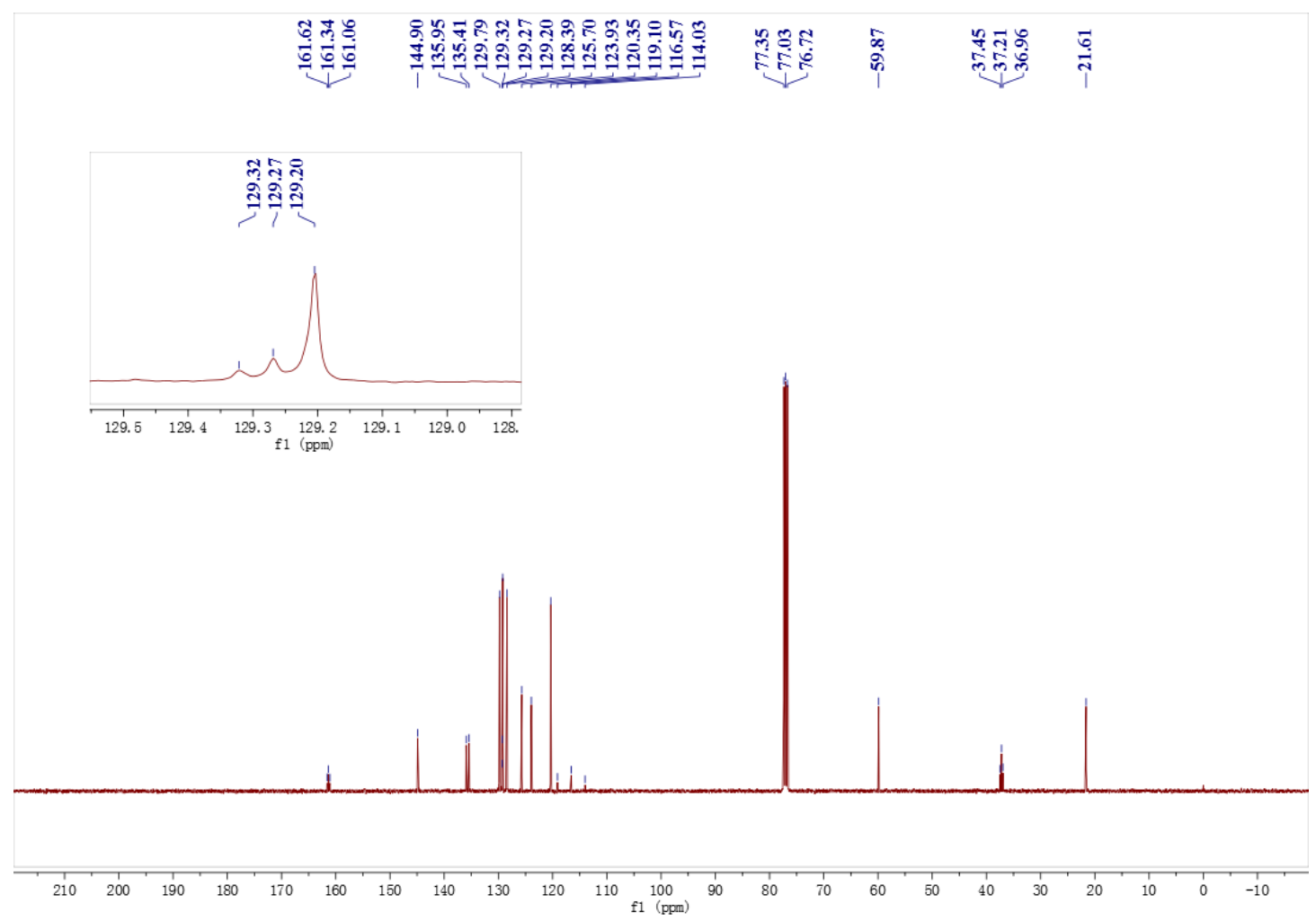


${ }^{1} \mathrm{H}$ NMR (400 MHz, CDCl $),{ }^{19} \mathrm{~F}\left\{{ }^{1} \mathrm{H}\right\}$ NMR $\left(375 \mathrm{MHz}, \mathrm{CDCl}_{3}\right),{ }^{13} \mathrm{C}\left\{{ }^{1} \mathrm{H}\right\}$ NMR (100 MHz, $\mathrm{CDCl}_{3}$ ) spectrum of product $6 \mathrm{f}$

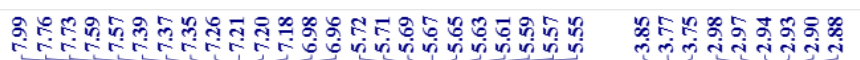
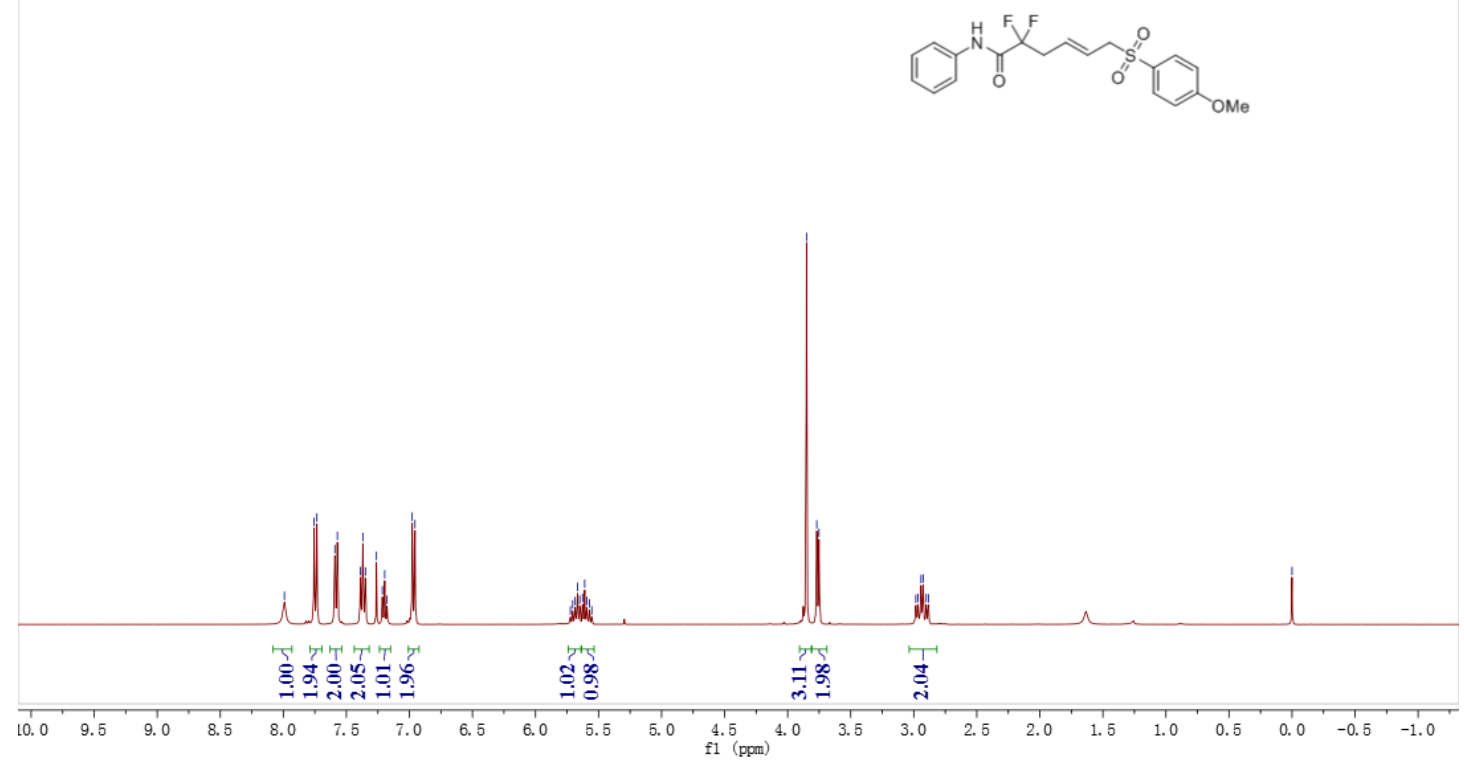

$\stackrel{\substack{+\dot{+}}}{\vec{i}}$

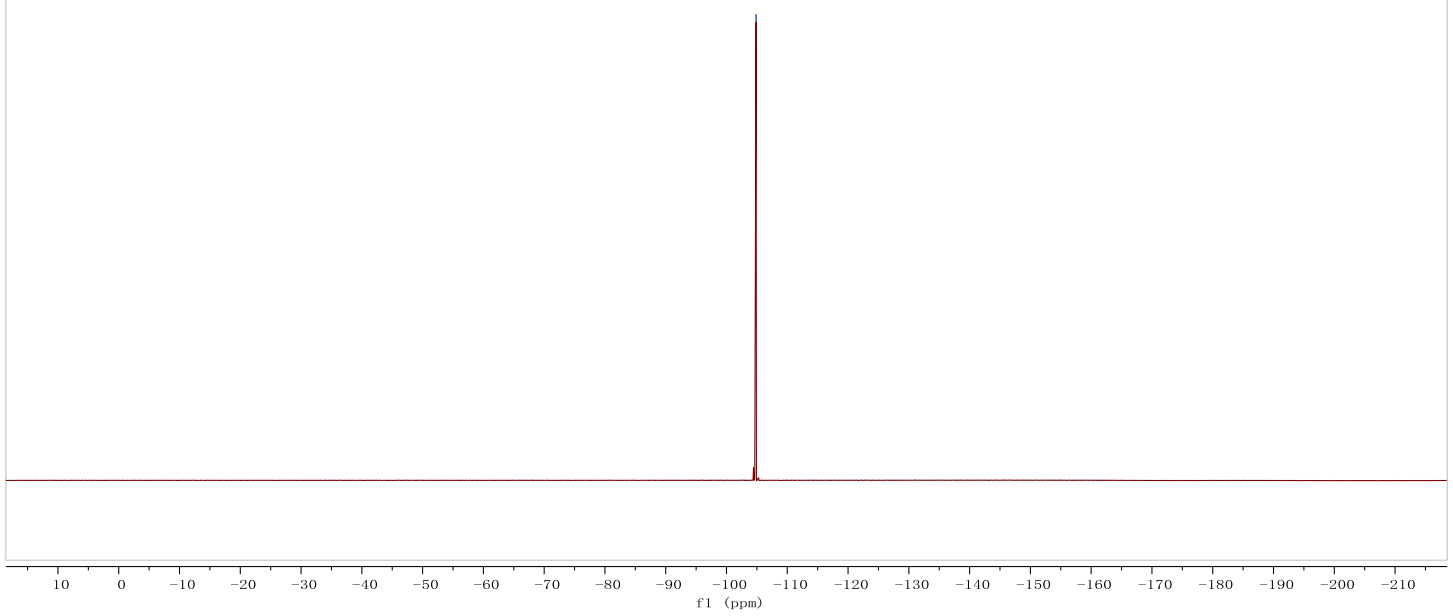




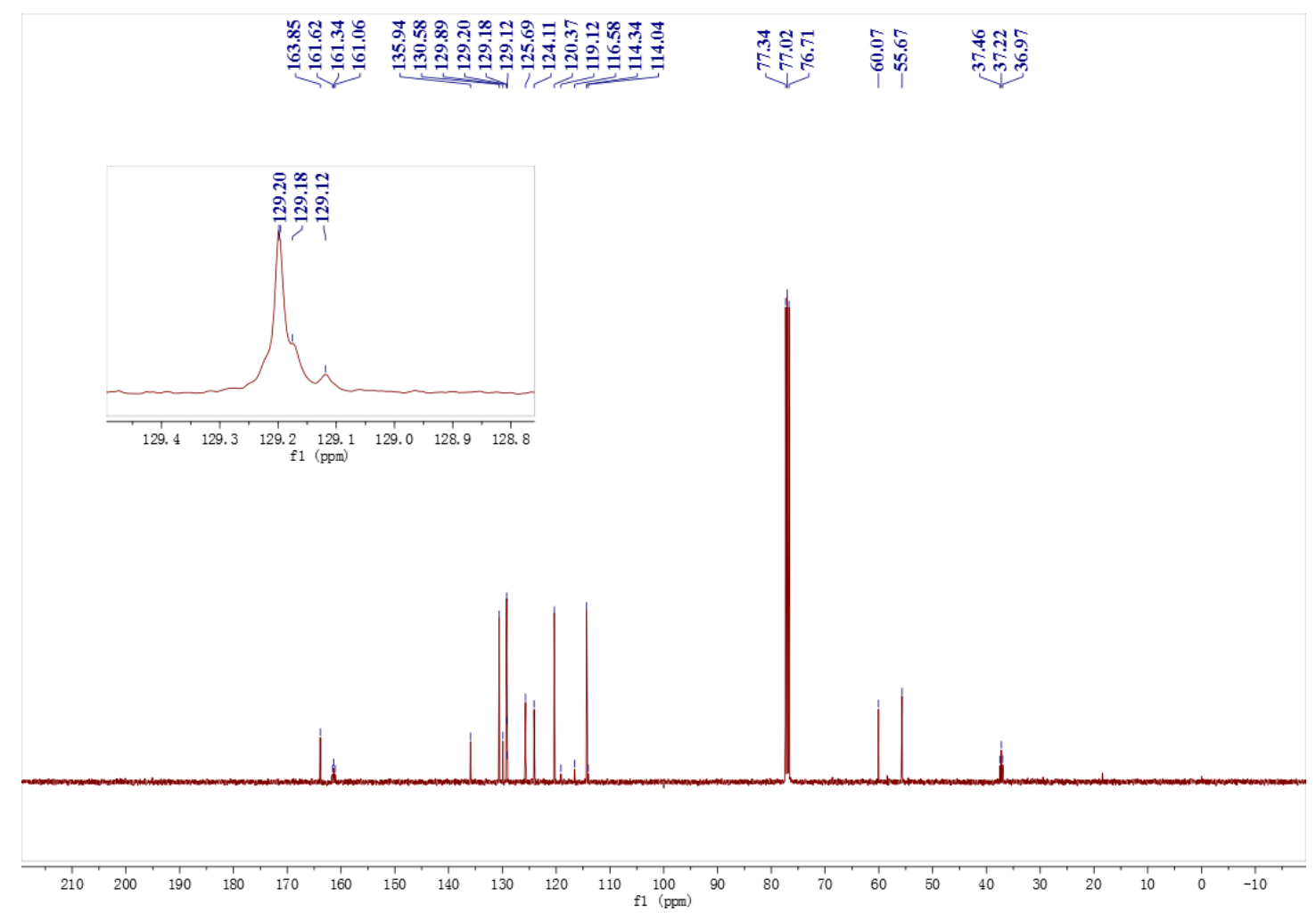


${ }^{1} \mathrm{H}$ NMR (400 MHz, CDCl $),{ }^{19} \mathrm{~F}\left\{{ }^{1} \mathrm{H}\right\}$ NMR $\left(375 \mathrm{MHz}, \mathrm{CDCl}_{3}\right),{ }^{13} \mathrm{C}\left\{{ }^{1} \mathrm{H}\right\}$ NMR (100 MHz, $\mathrm{CDCl}_{3}$ ) spectrum of product $6 \mathrm{~g}$

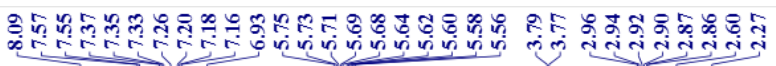

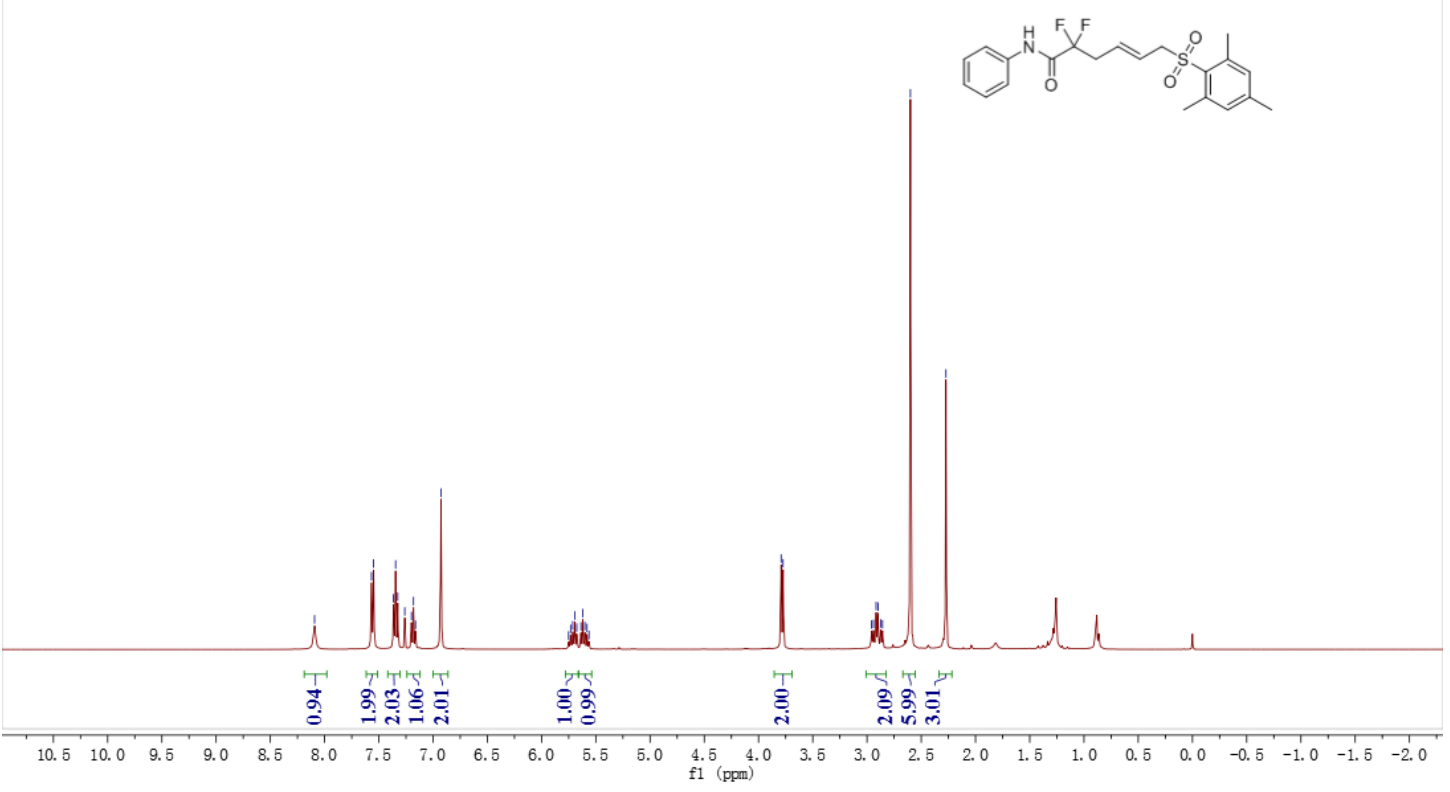

है

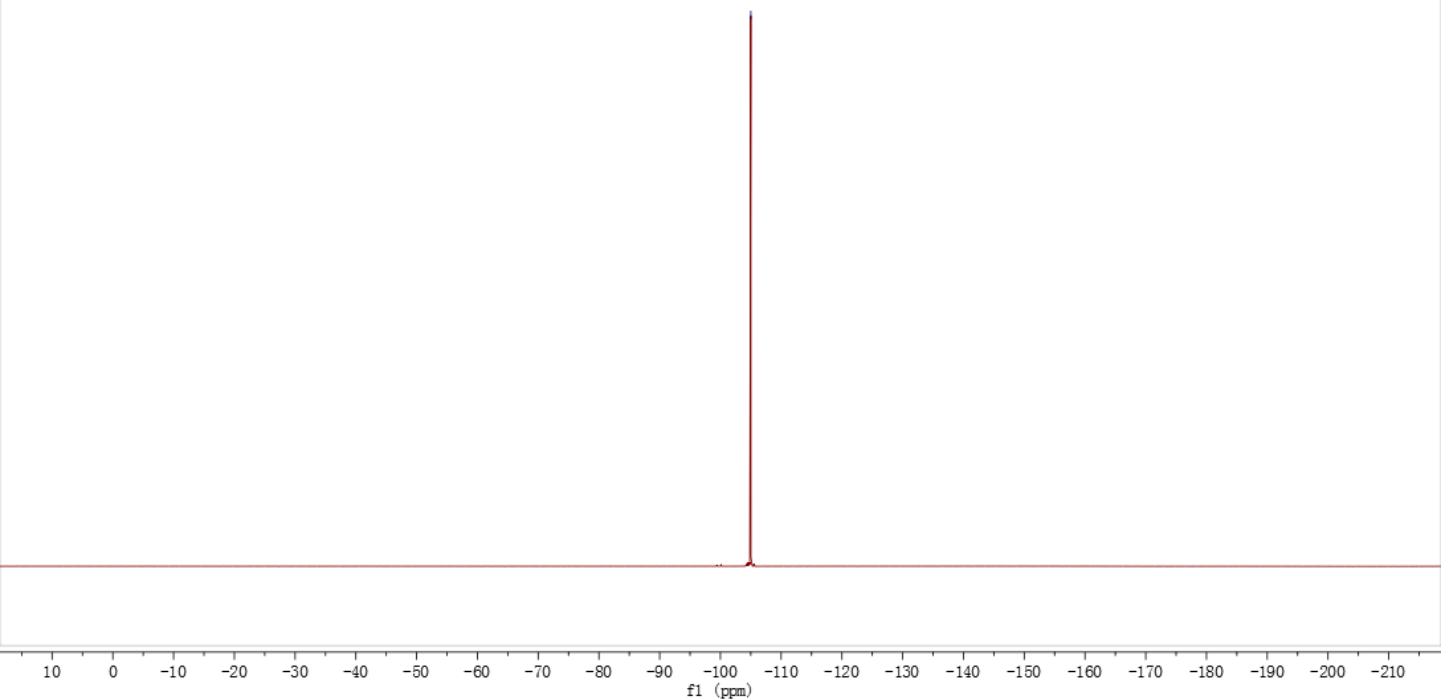




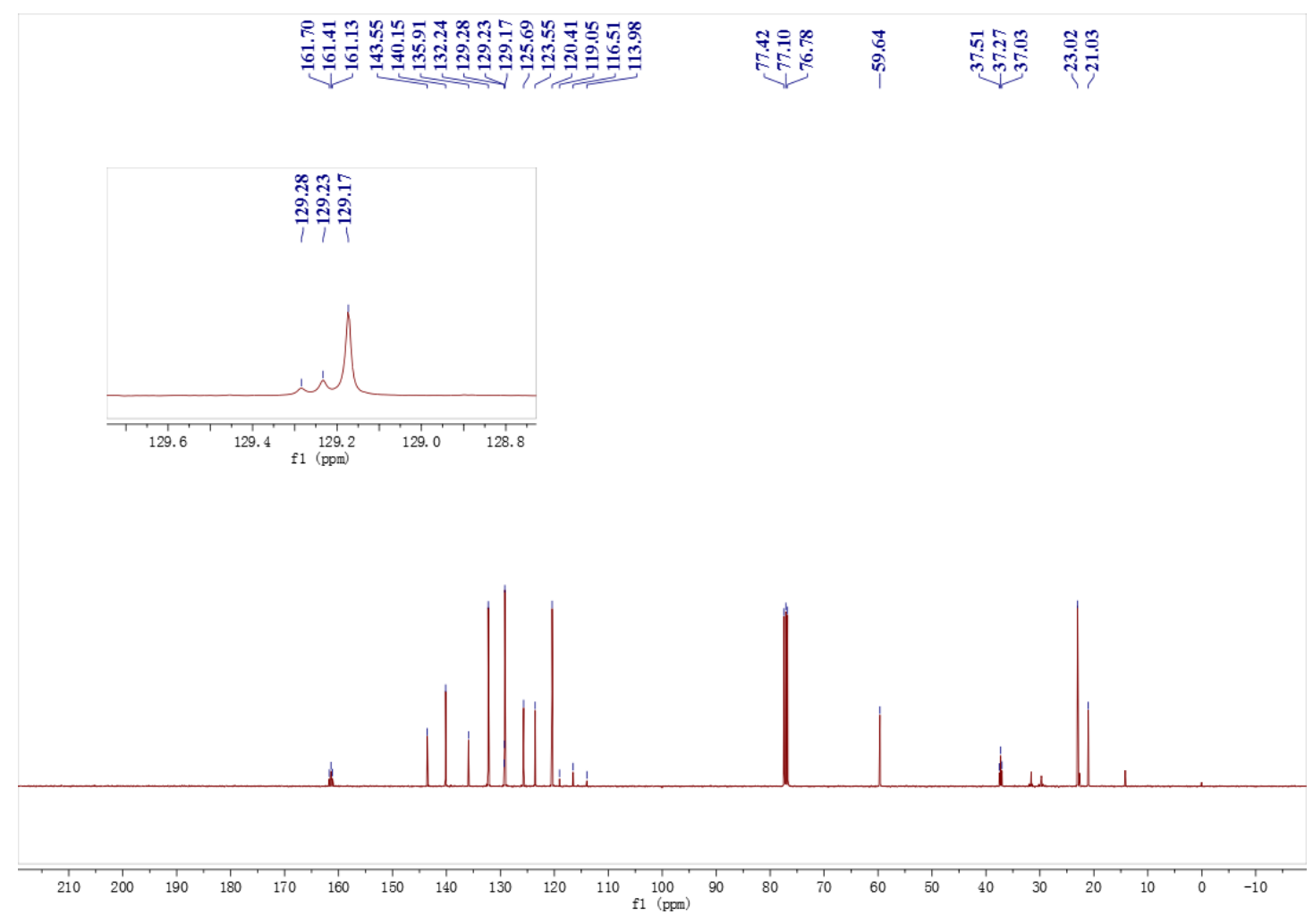


${ }^{1} \mathrm{H}$ NMR (400 MHz, CDCl $),{ }^{19} \mathrm{~F}\left\{{ }^{1} \mathrm{H}\right\}$ NMR $\left(375 \mathrm{MHz}, \mathrm{CDCl}_{3}\right),{ }^{13} \mathrm{C}\left\{{ }^{1} \mathrm{H}\right\}$ NMR (100 MHz, $\mathrm{CDCl}_{3}$ ) spectrum of product $6 \mathrm{~h}$

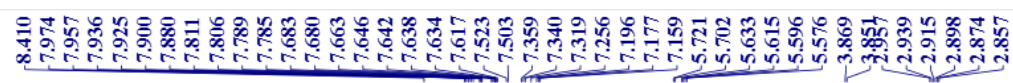
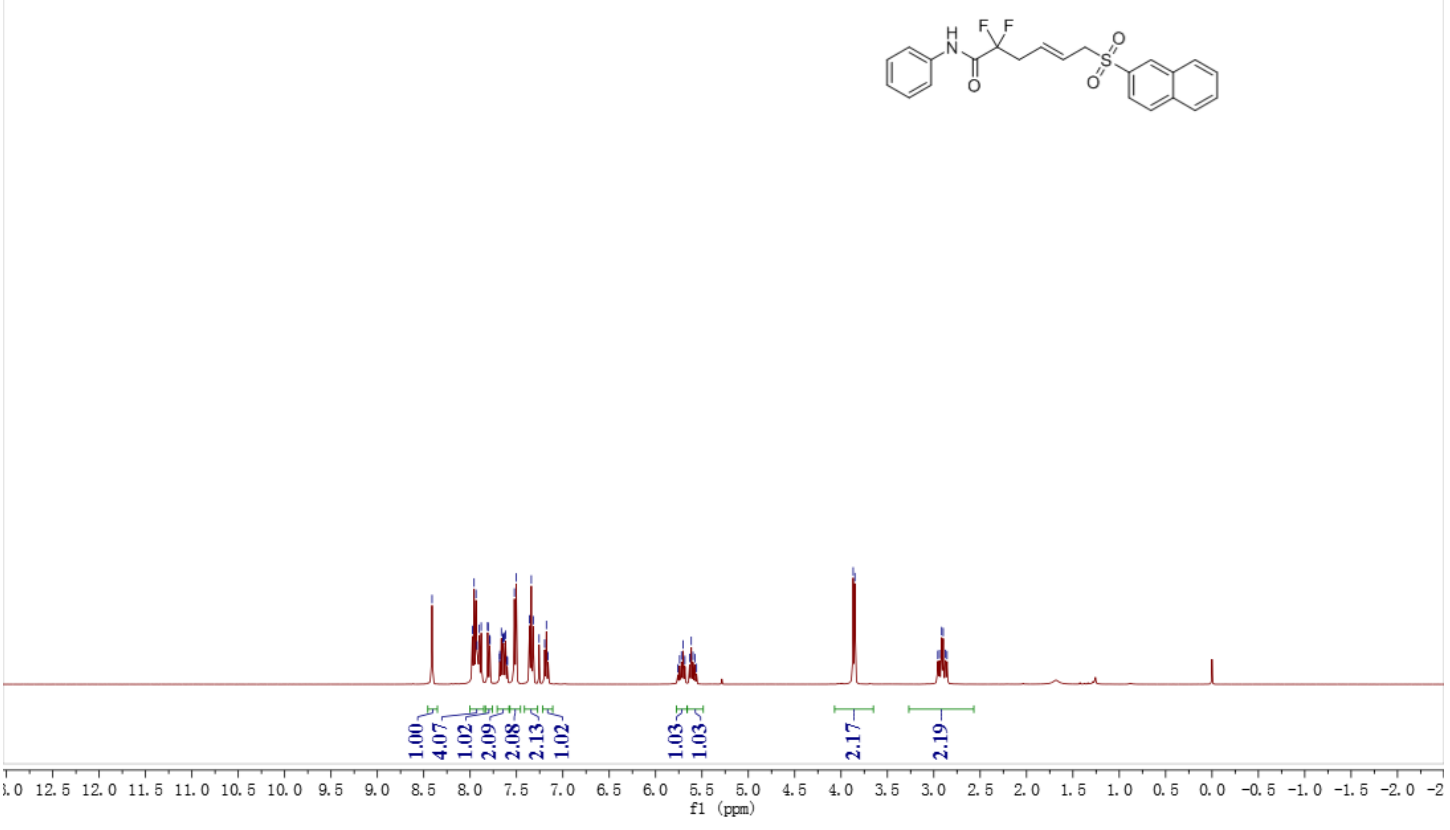

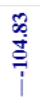

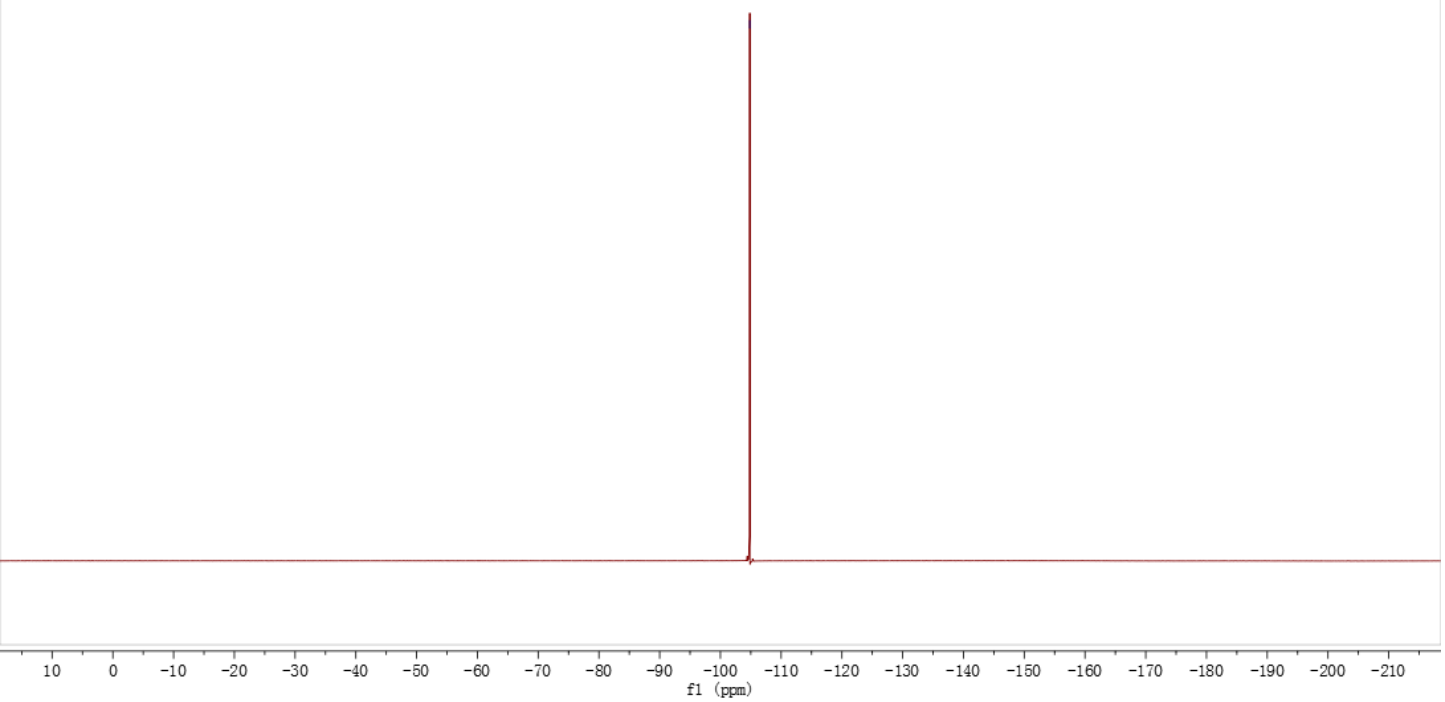




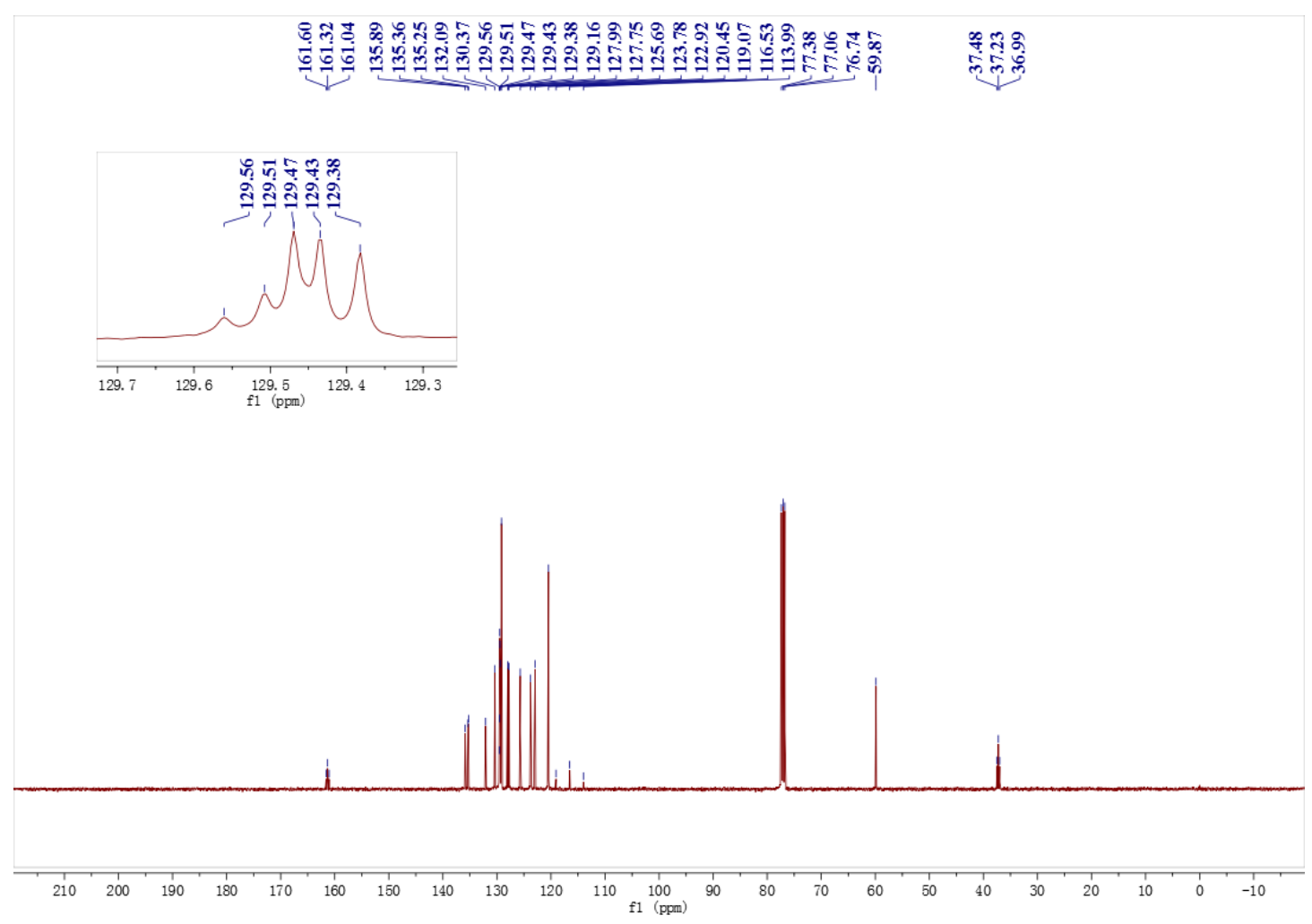


${ }^{1} \mathrm{H}$ NMR (400 MHz, CDCl $),{ }^{19} \mathrm{~F}\left\{{ }^{1} \mathrm{H}\right\}$ NMR $\left(375 \mathrm{MHz}, \mathrm{CDCl}_{3}\right),{ }^{13} \mathrm{C}\left\{{ }^{1} \mathrm{H}\right\}$ NMR (100 MHz, $\mathrm{CDCl}_{3}$ ) spectrum of product $6 \mathbf{i}$

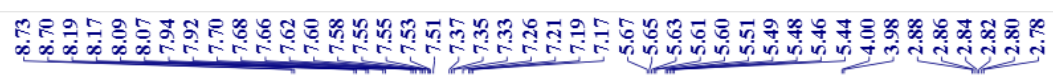

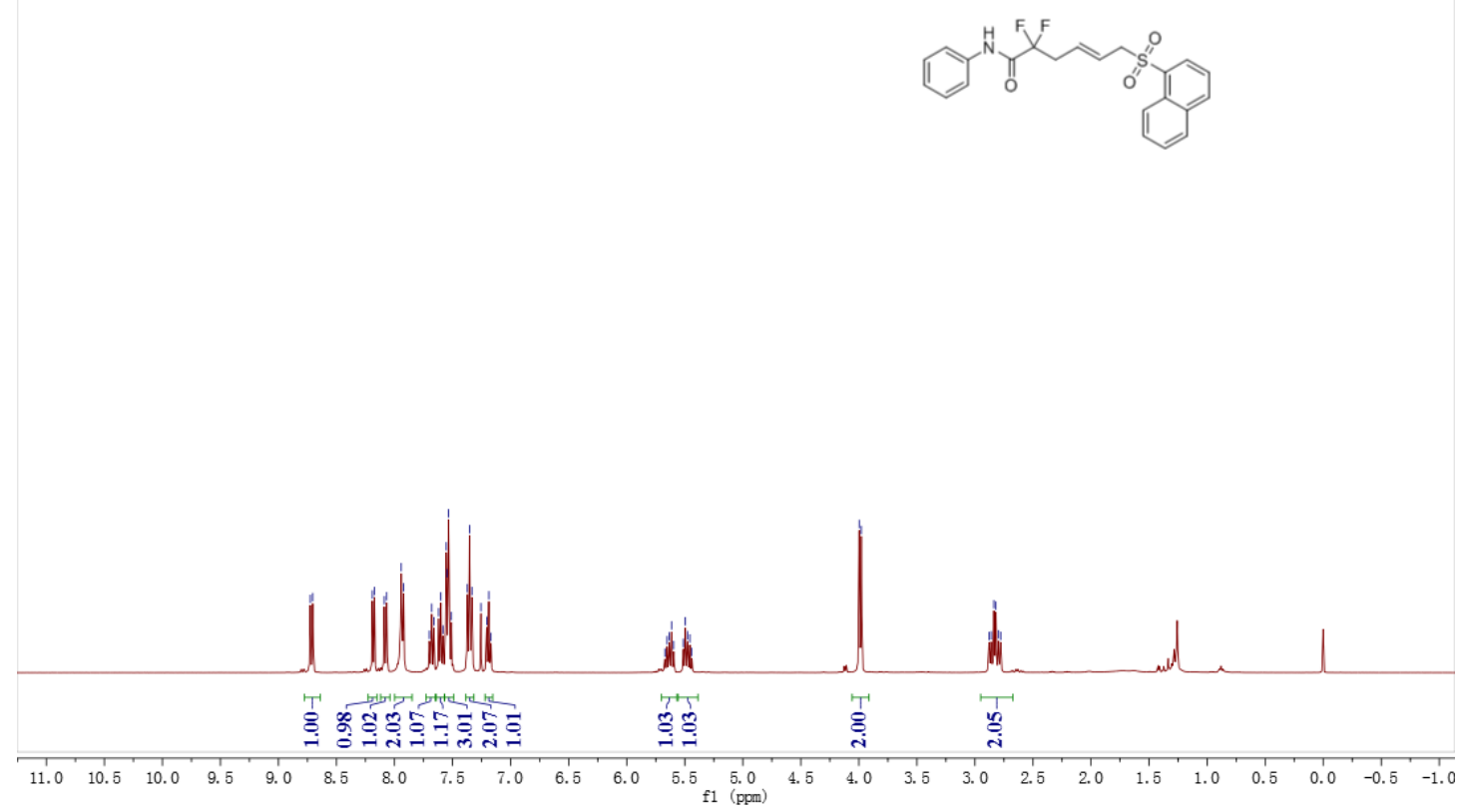

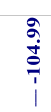

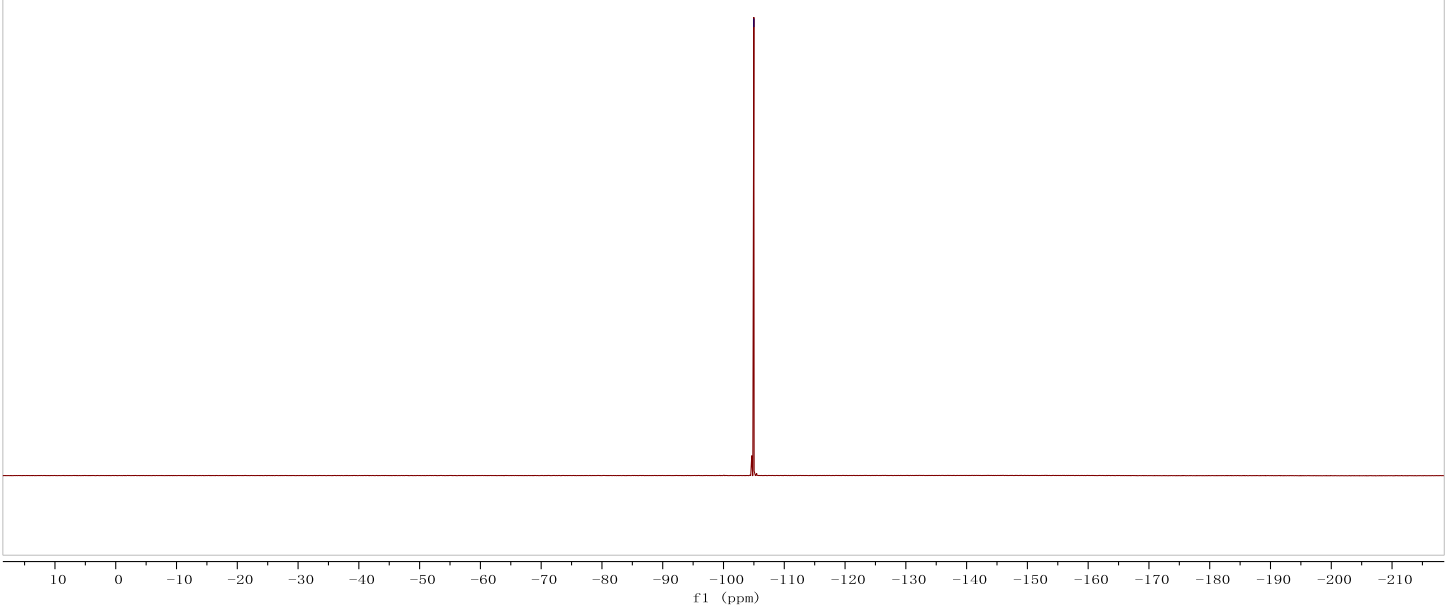




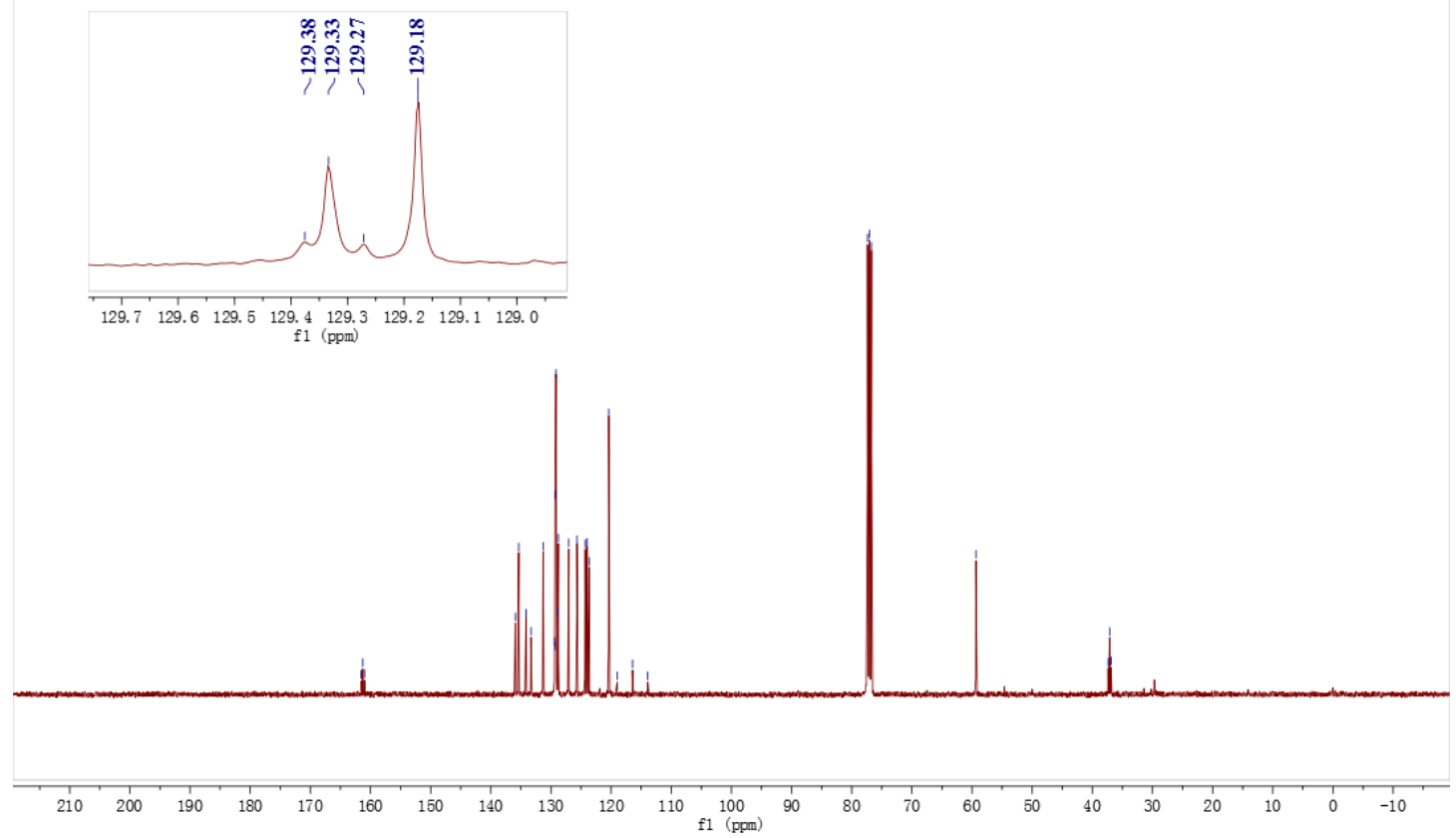


${ }^{1} \mathrm{H}$ NMR (400 MHz, CDCl $),{ }^{19} \mathrm{~F}\left\{{ }^{1} \mathrm{H}\right\}$ NMR $\left(375 \mathrm{MHz}, \mathrm{CDCl}_{3}\right),{ }^{13} \mathrm{C}\left\{{ }^{1} \mathrm{H}\right\}$ NMR (100 MHz, $\mathrm{CDCl}_{3}$ ) spectrum of product $\mathbf{6 j}$

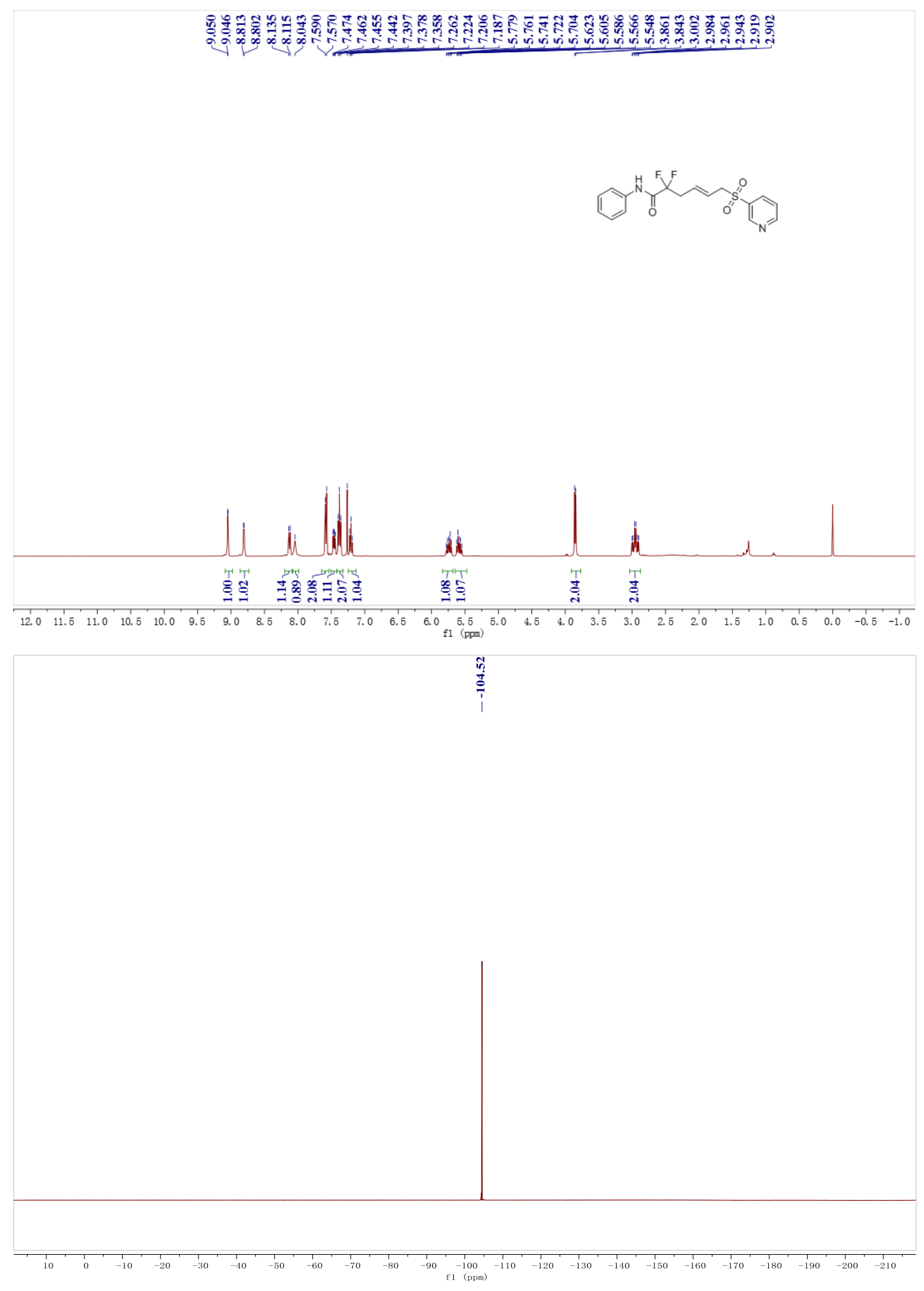




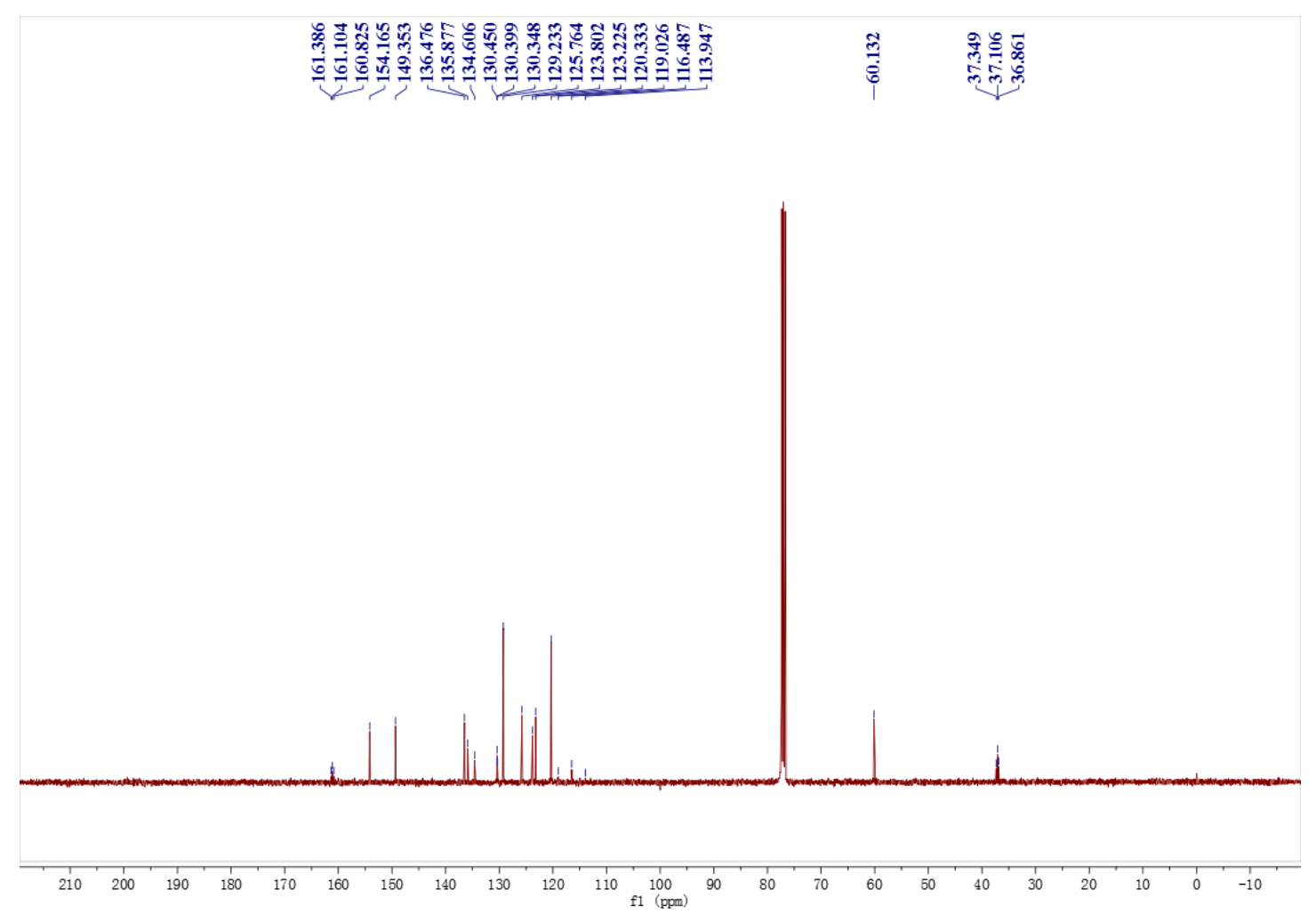

S96 
${ }^{1} \mathrm{H}$ NMR (400 MHz, CDCl $),{ }^{19} \mathrm{~F}\left\{{ }^{1} \mathrm{H}\right\}$ NMR $\left(375 \mathrm{MHz}, \mathrm{CDCl}_{3}\right),{ }^{13} \mathrm{C}\left\{{ }^{1} \mathrm{H}\right\}$ NMR (100 MHz, $\mathrm{CDCl}_{3}$ ) spectrum of product $6 \mathrm{k}$

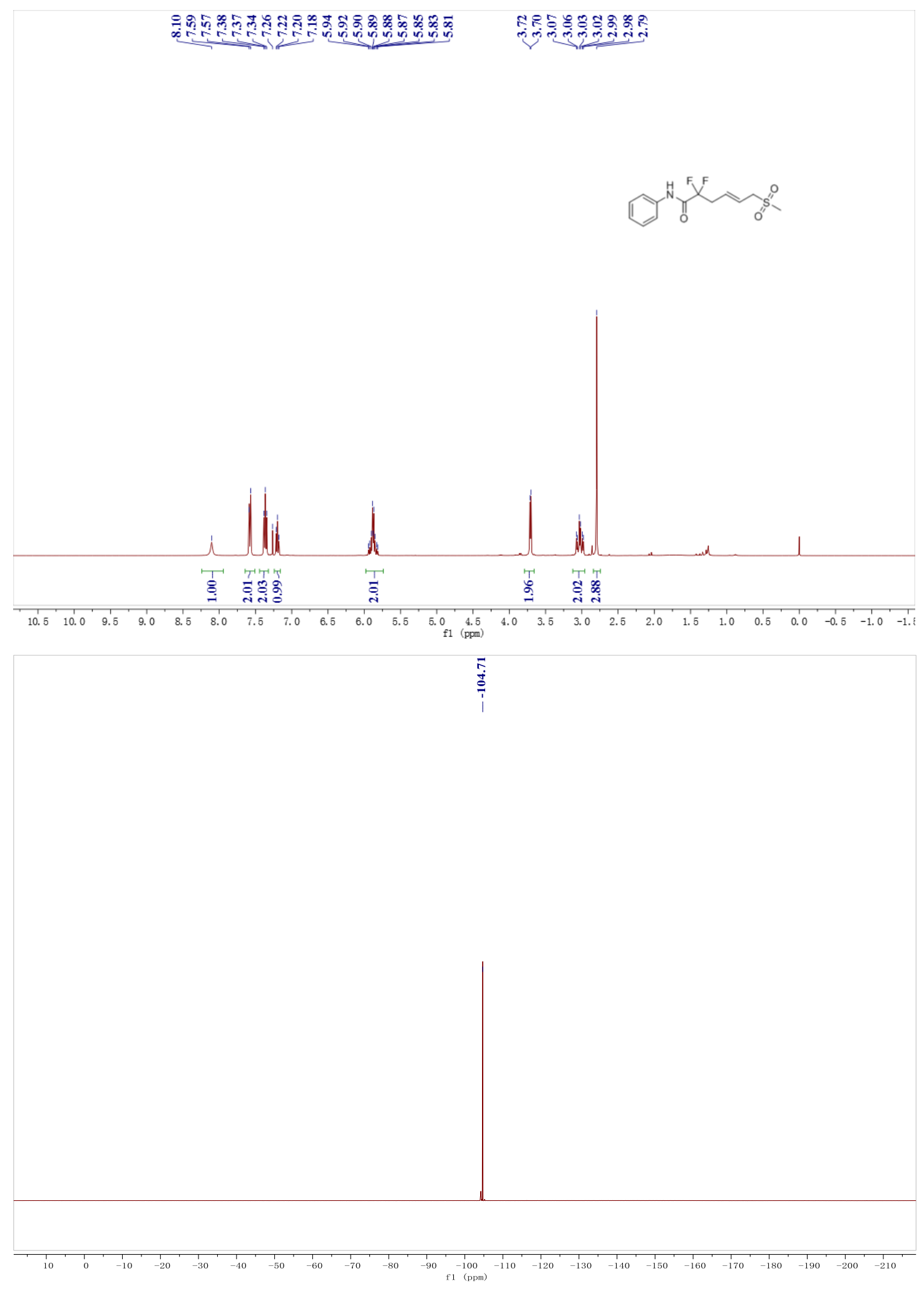




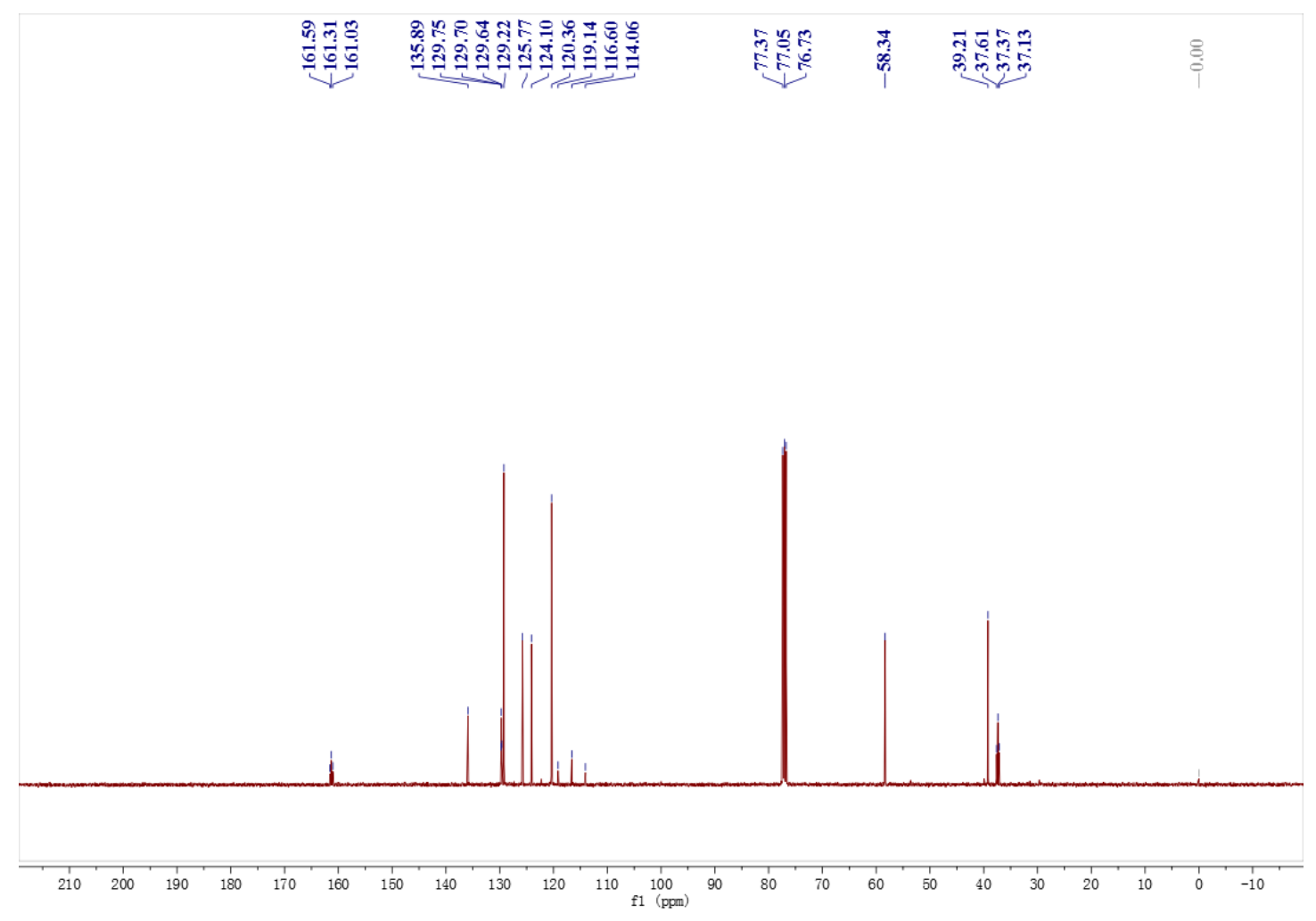


${ }^{1} \mathrm{H}$ NMR (400 MHz, CDCl $),{ }^{19} \mathrm{~F}\left\{{ }^{1} \mathrm{H}\right\}$ NMR $\left(375 \mathrm{MHz}, \mathrm{CDCl}_{3}\right),{ }^{13} \mathrm{C}\left\{{ }^{1} \mathrm{H}\right\}$ NMR (100 MHz, $\mathrm{CDCl}_{3}$ ) spectrum of product $6 \mathrm{l}$

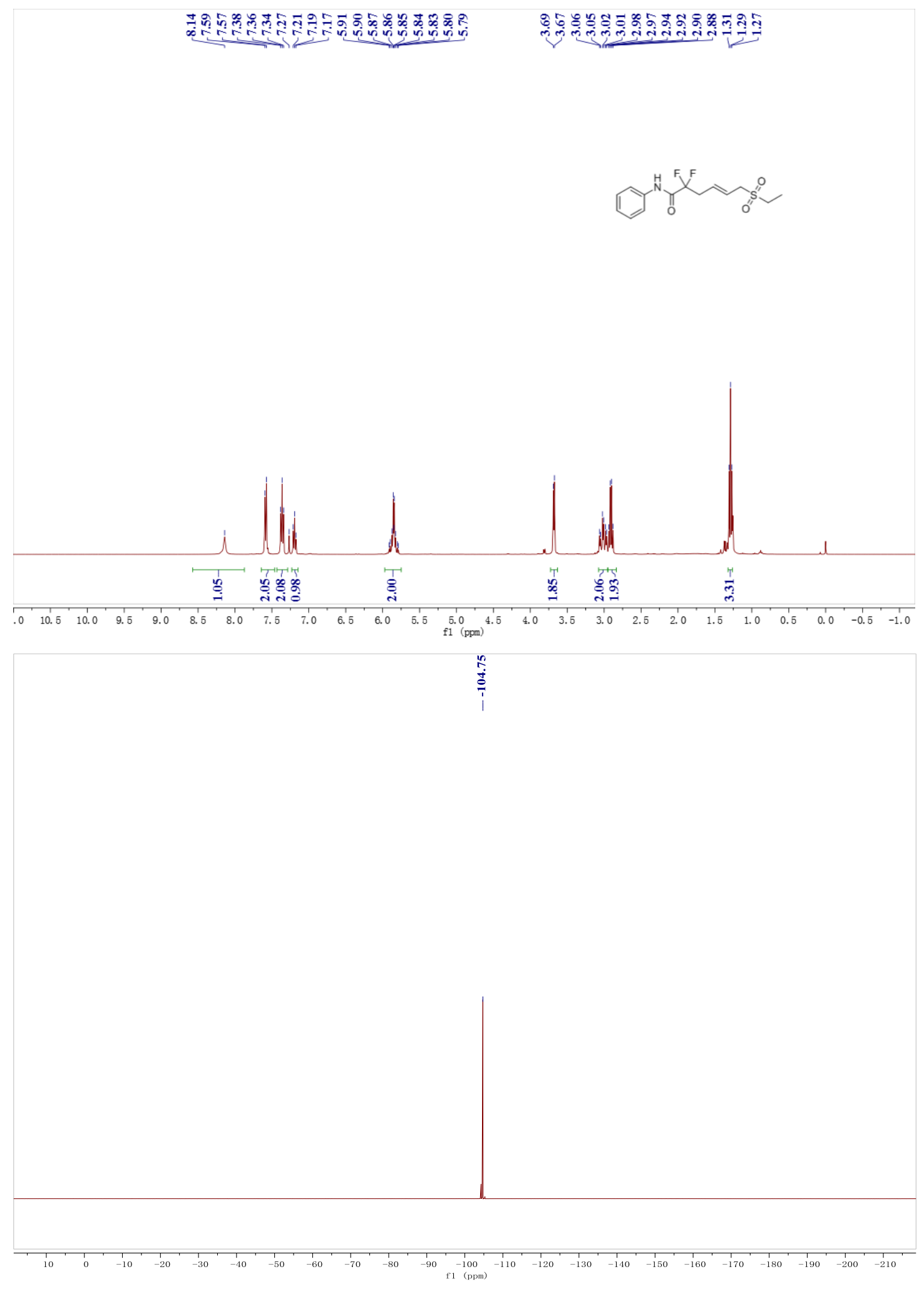




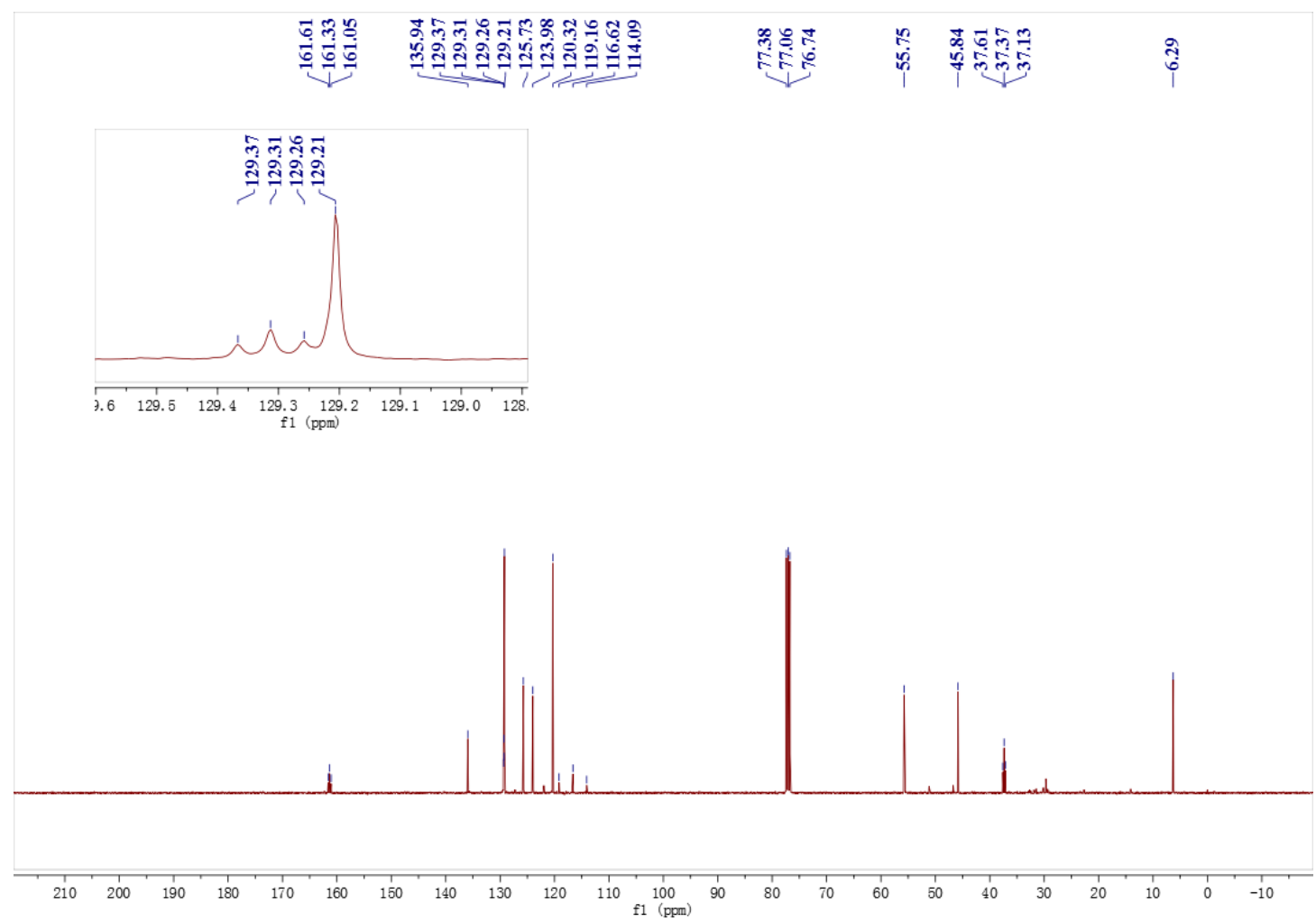


${ }^{1} \mathrm{H}$ NMR (400 MHz, $\left.\mathrm{CDCl}_{3}\right),{ }^{19} \mathrm{~F}\left\{{ }^{1} \mathrm{H}\right\}$ NMR $\left(375 \mathrm{MHz}, \mathrm{CDCl}_{3}\right),{ }^{13} \mathrm{C}\left\{{ }^{1} \mathrm{H}\right\}$ NMR (100 MHz, $\mathrm{CDCl}_{3}$ ) spectrum of product $6 \mathrm{~m}$

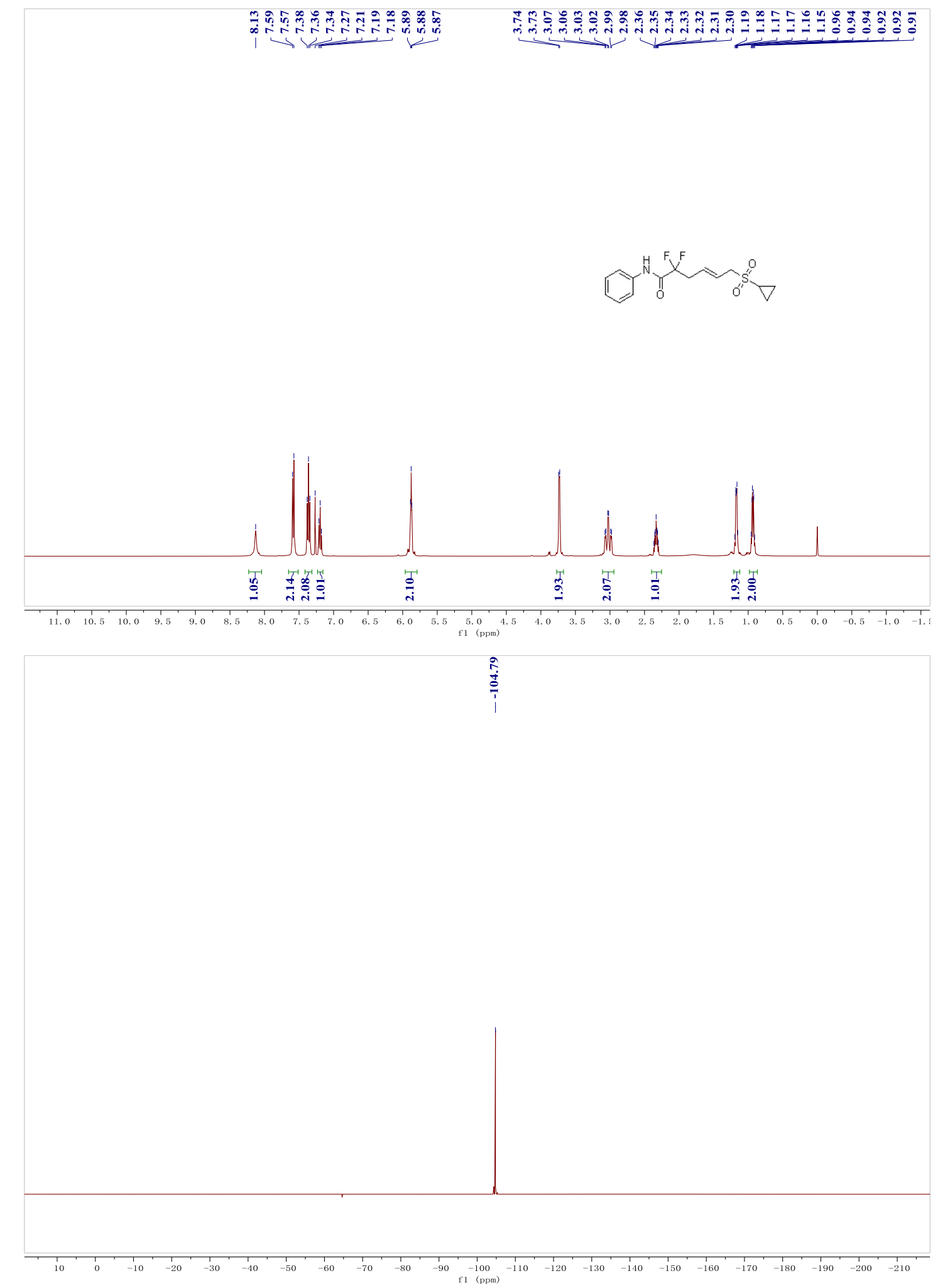




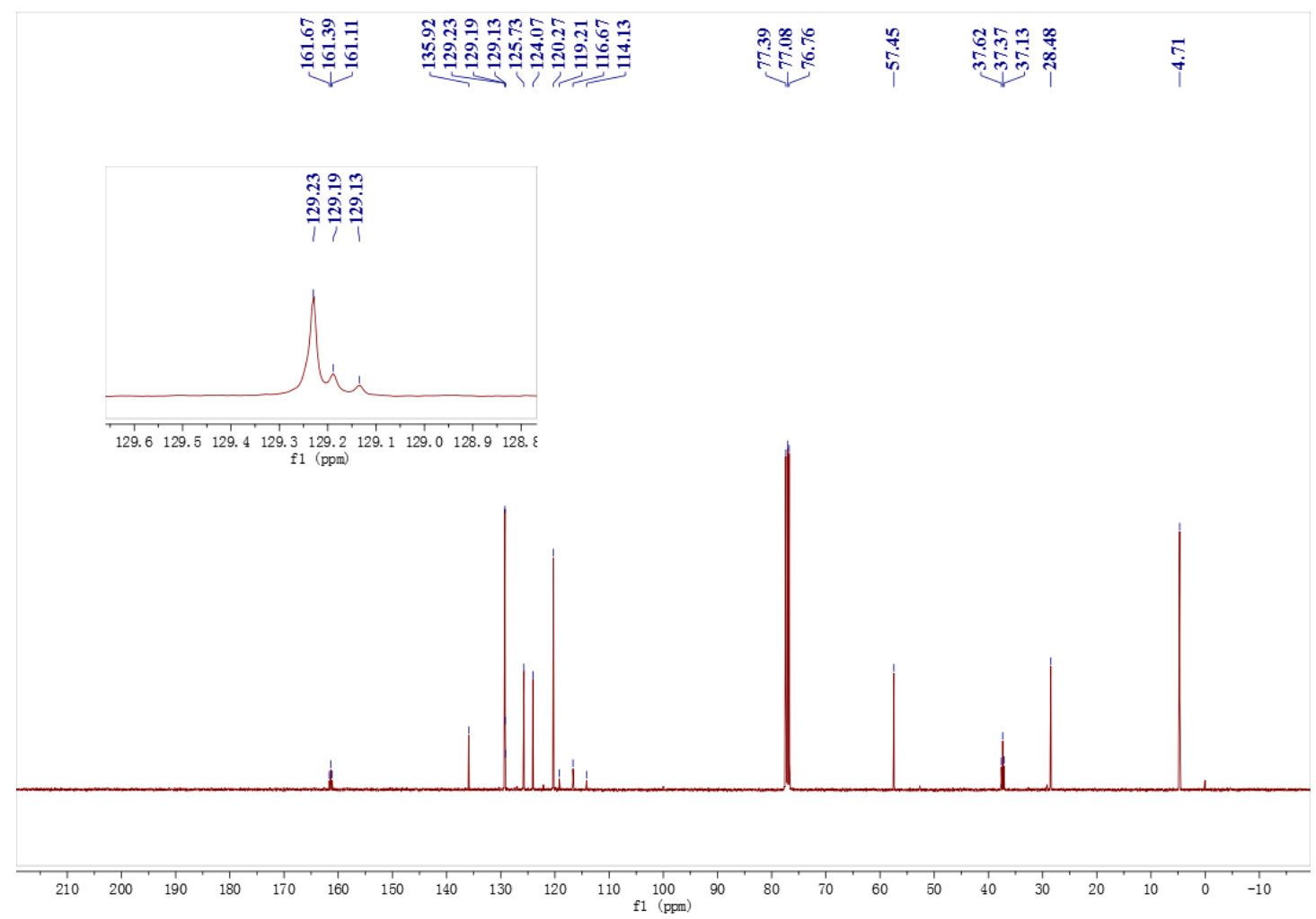


${ }^{1} \mathrm{H}$ NMR (400 MHz, CDCl $),{ }^{19} \mathrm{~F}\left\{{ }^{1} \mathrm{H}\right\}$ NMR $\left(375 \mathrm{MHz}, \mathrm{CDCl}_{3}\right),{ }^{13} \mathrm{C}\left\{{ }^{1} \mathrm{H}\right\}$ NMR (100 MHz, $\mathrm{CDCl}_{3}$ ) spectrum of product $6 \mathrm{n}$

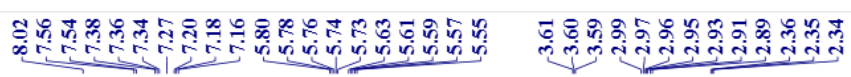
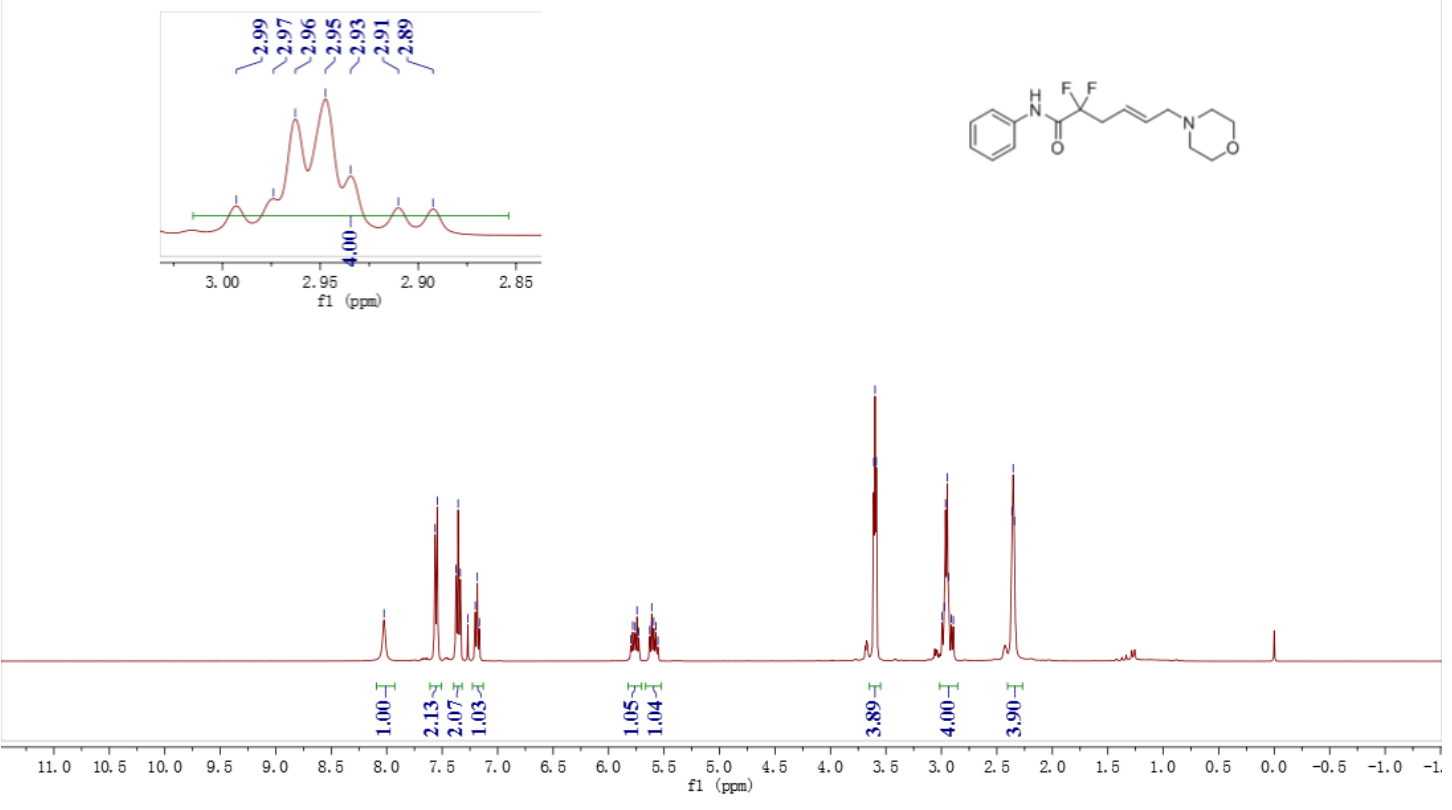
$\stackrel{7}{i}$
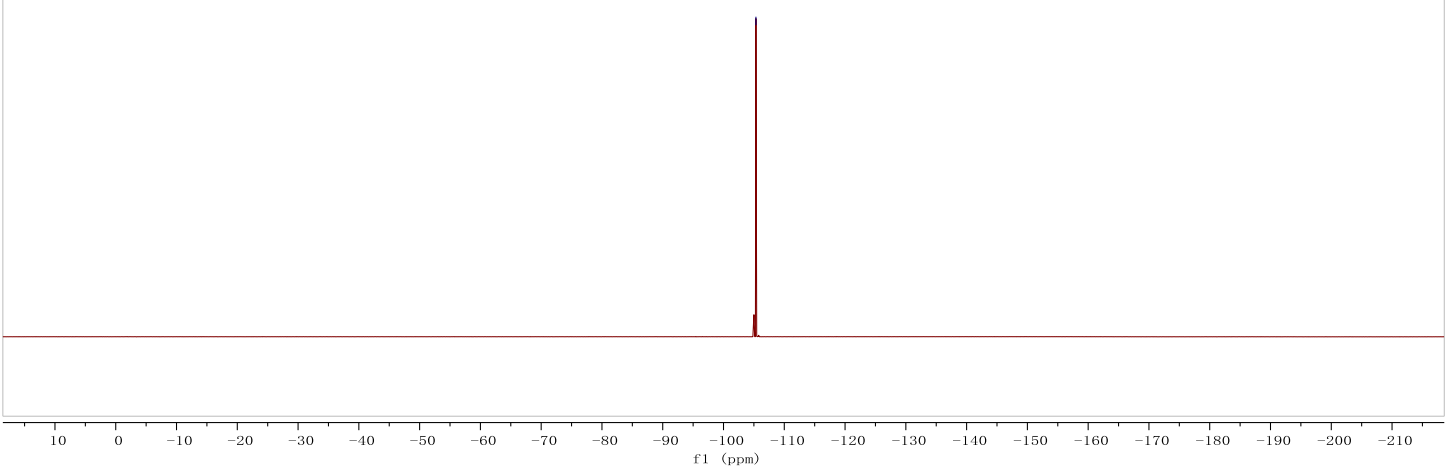


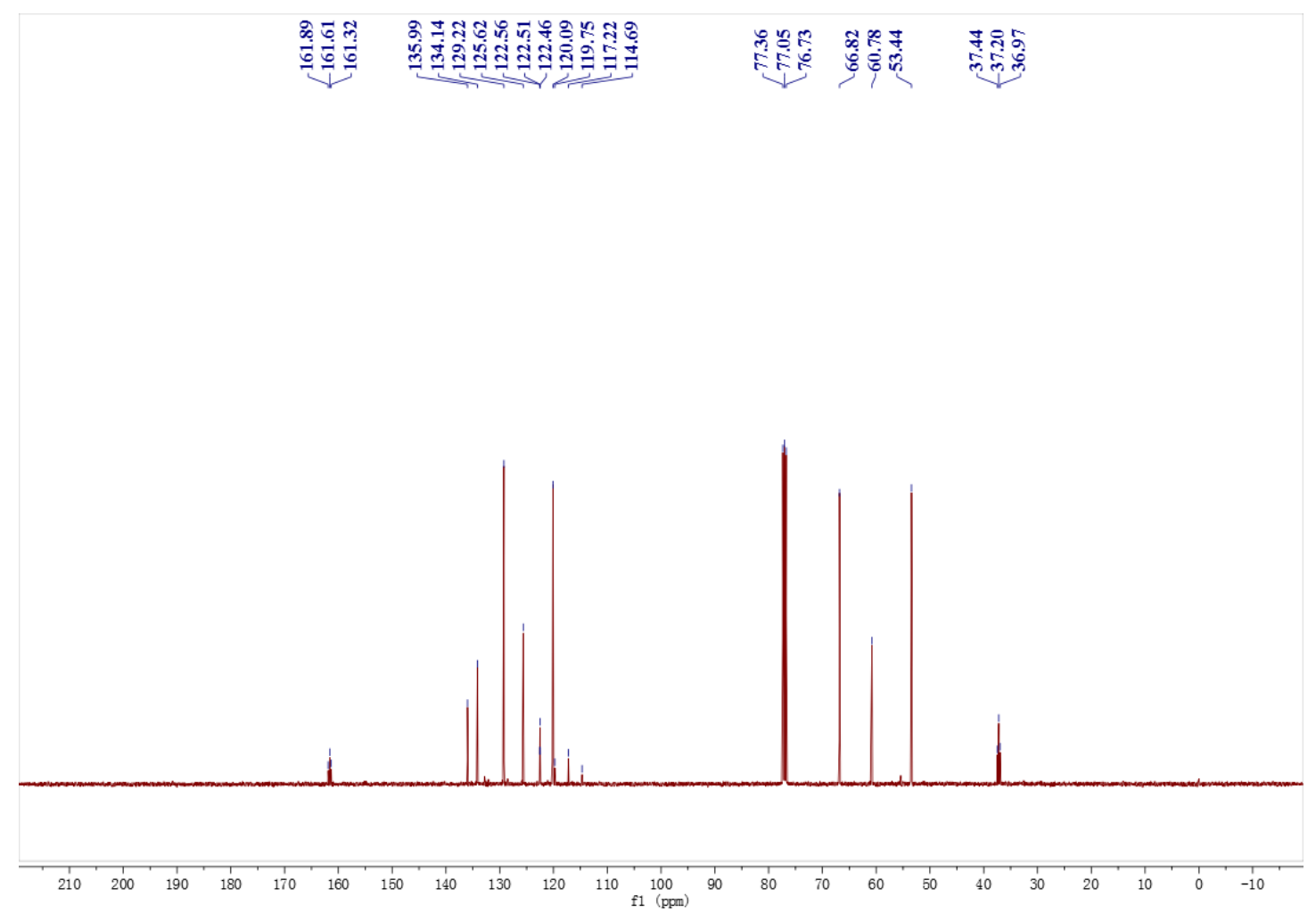


${ }^{1} \mathrm{H}$ NMR (400 MHz, CDCl $),{ }^{19} \mathrm{~F}\left\{{ }^{1} \mathrm{H}\right\}$ NMR $\left(375 \mathrm{MHz}, \mathrm{CDCl}_{3}\right),{ }^{13} \mathrm{C}\left\{{ }^{1} \mathrm{H}\right\}$ NMR (100 MHz, $\mathrm{CDCl}_{3}$ ) spectrum of product 60

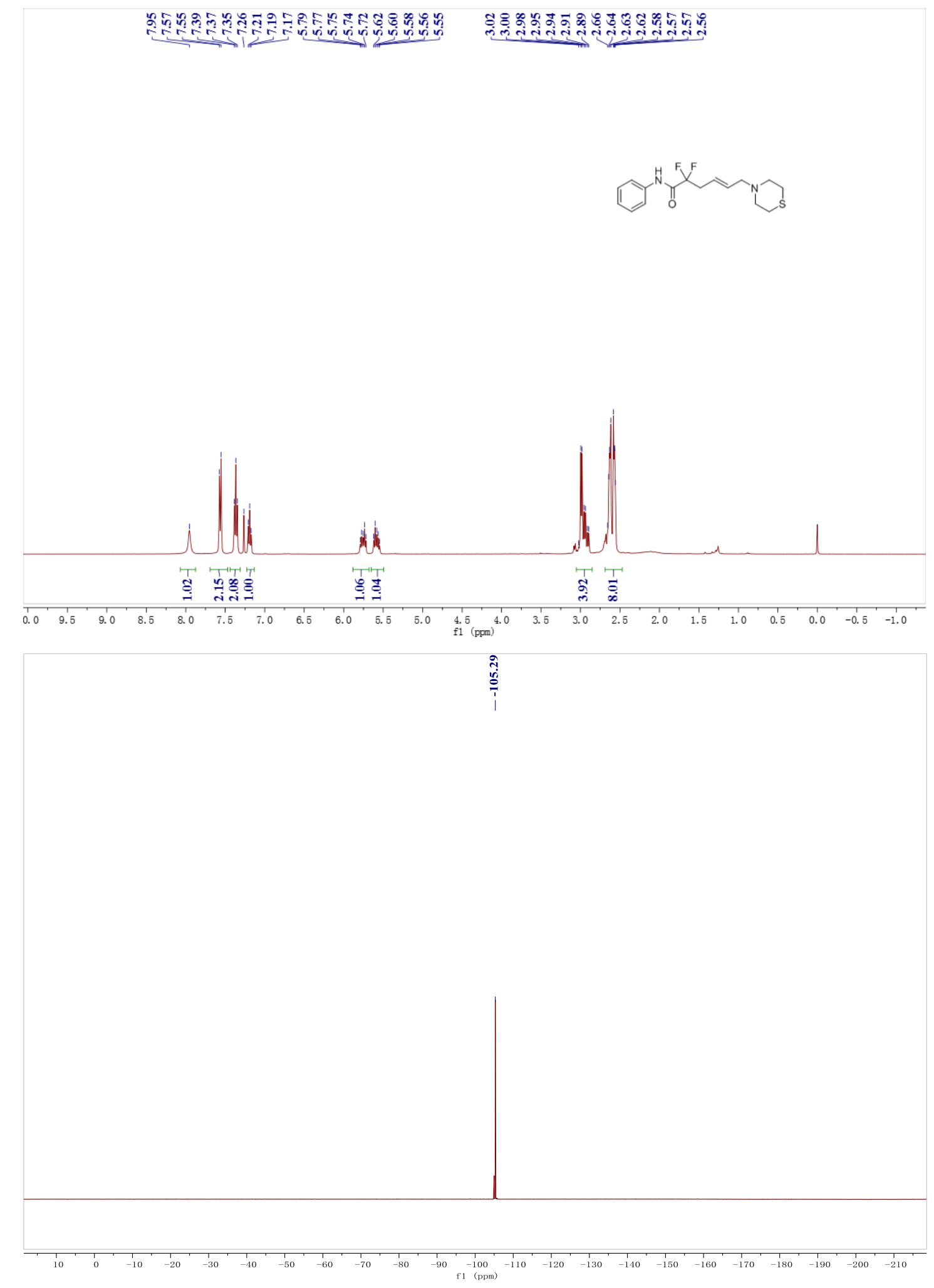




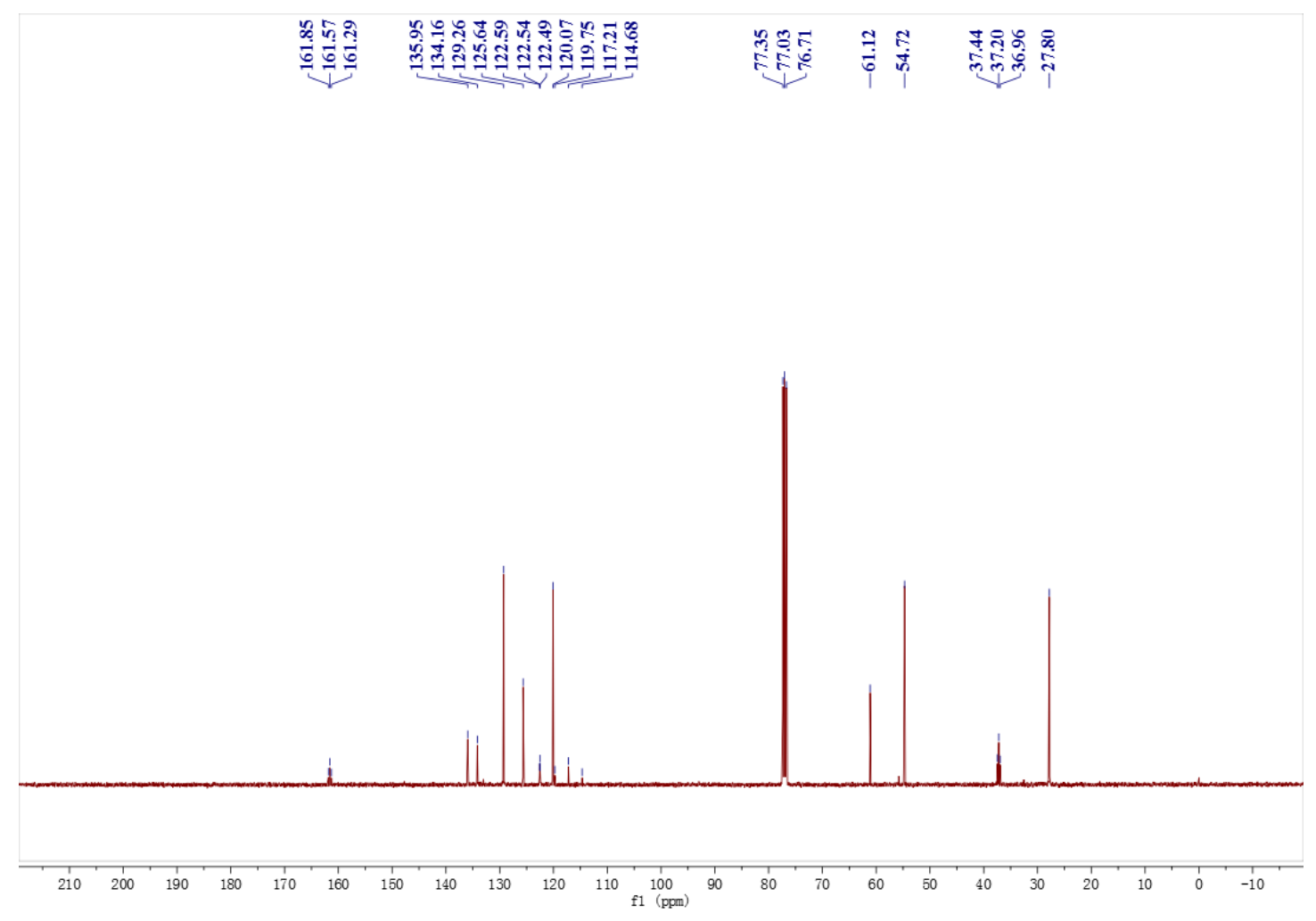


${ }^{1} \mathrm{H}$ NMR (400 MHz, $\left.\mathrm{CDCl}_{3}\right),{ }^{19} \mathrm{~F}\left\{{ }^{1} \mathrm{H}\right\}$ NMR $\left(375 \mathrm{MHz}, \mathrm{CDCl}_{3}\right),{ }^{13} \mathrm{C}\left\{{ }^{1} \mathrm{H}\right\}$ NMR (100 MHz, $\mathrm{CDCl}_{3}$ ) spectrum of product $6 \mathrm{p}$

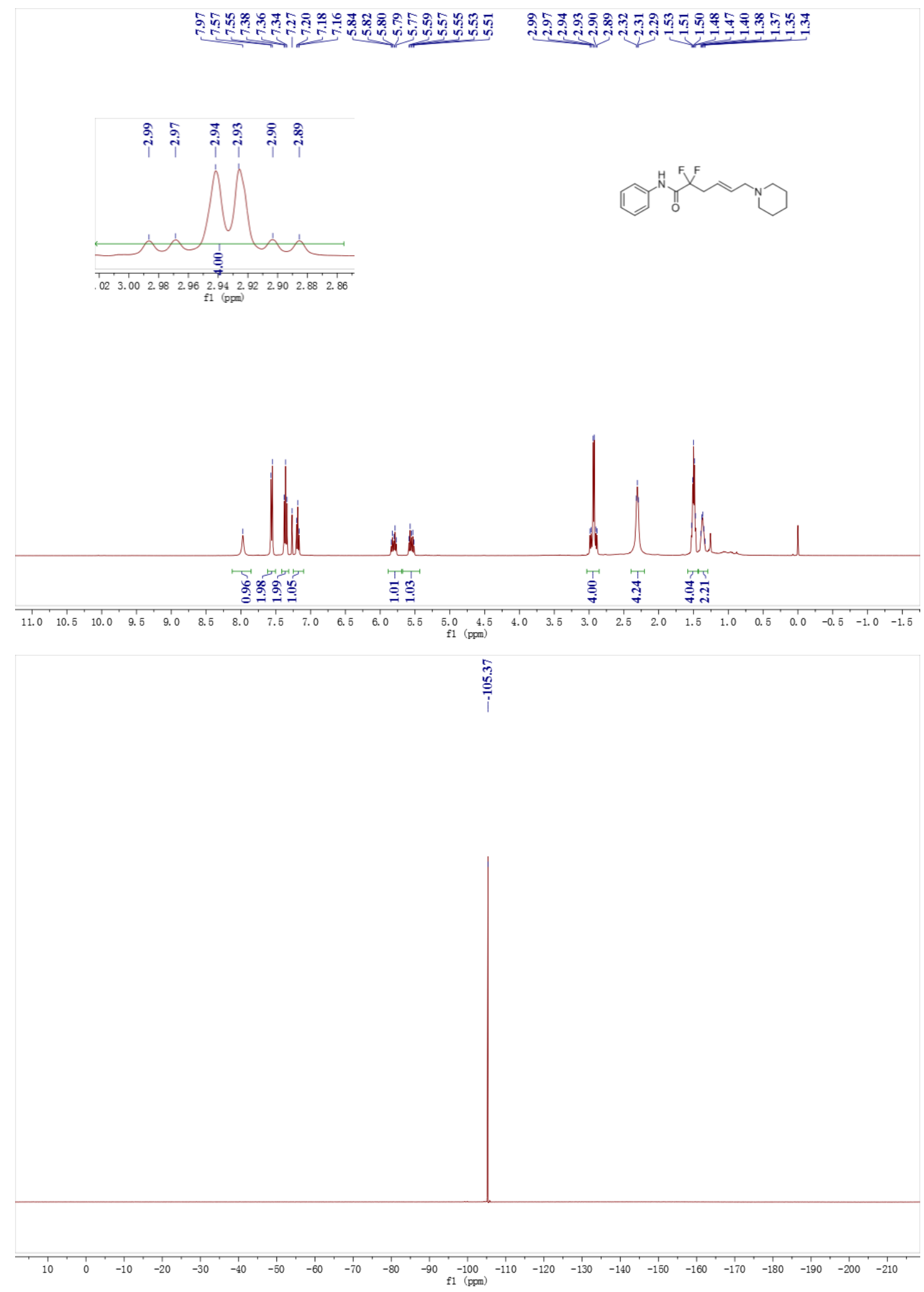




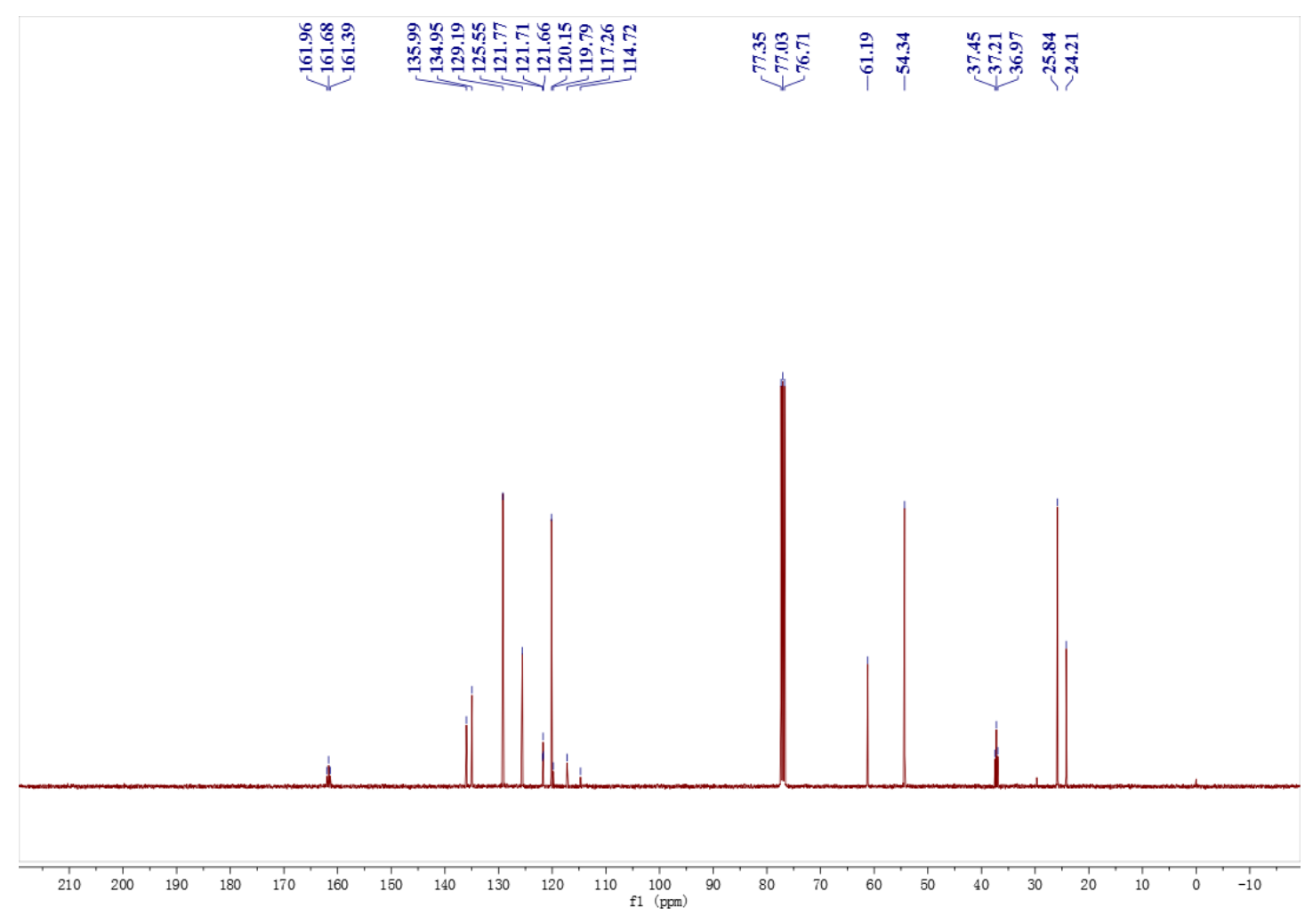


${ }^{1} \mathrm{H}$ NMR (400 MHz, CDCl $),{ }^{19} \mathrm{~F}\left\{{ }^{1} \mathrm{H}\right\}$ NMR $\left(375 \mathrm{MHz}, \mathrm{CDCl}_{3}\right),{ }^{13} \mathrm{C}\left\{{ }^{1} \mathrm{H}\right\}$ NMR (100 MHz, $\mathrm{CDCl}_{3}$ ) spectrum of product $\mathbf{6 q}$

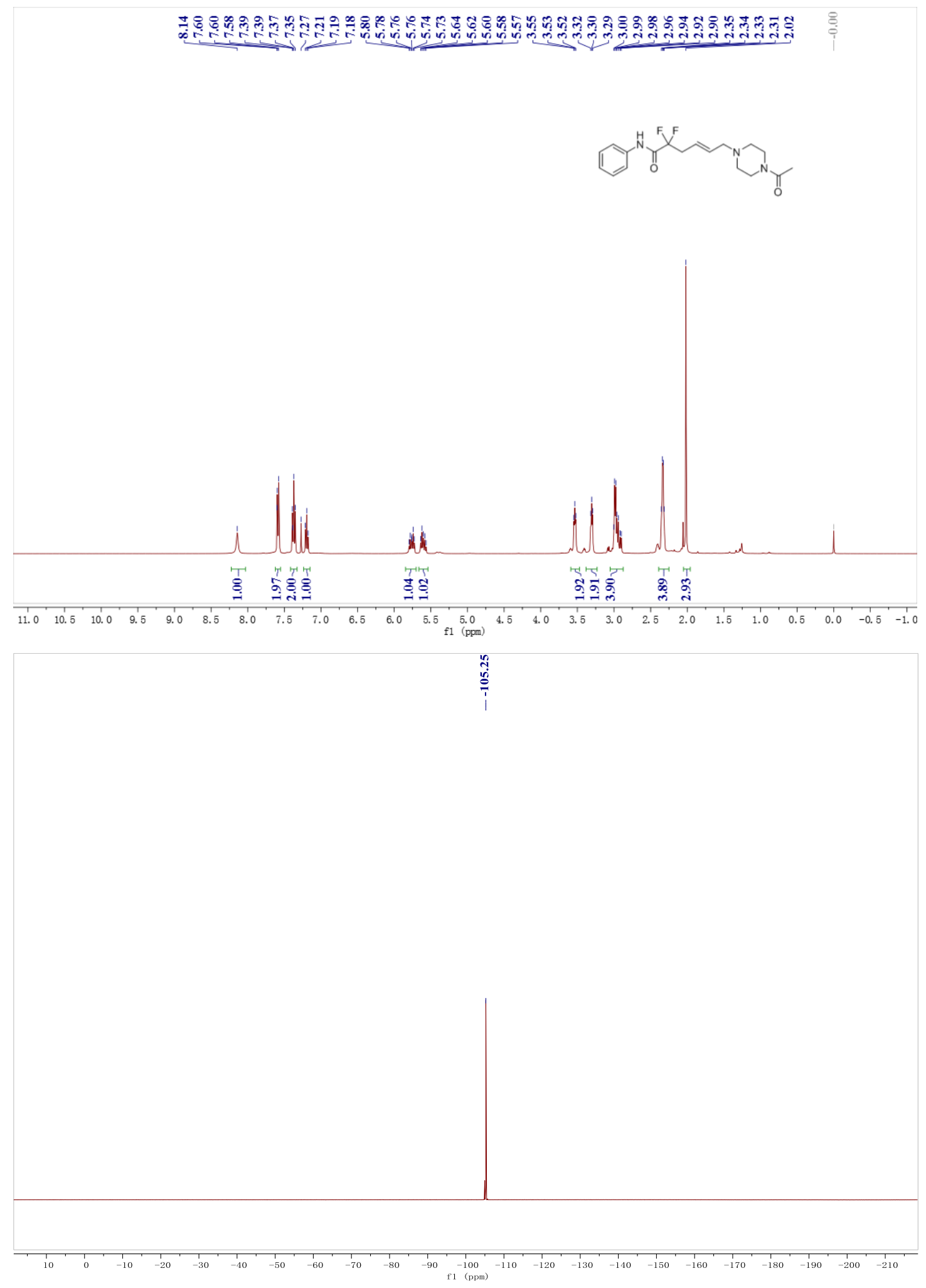




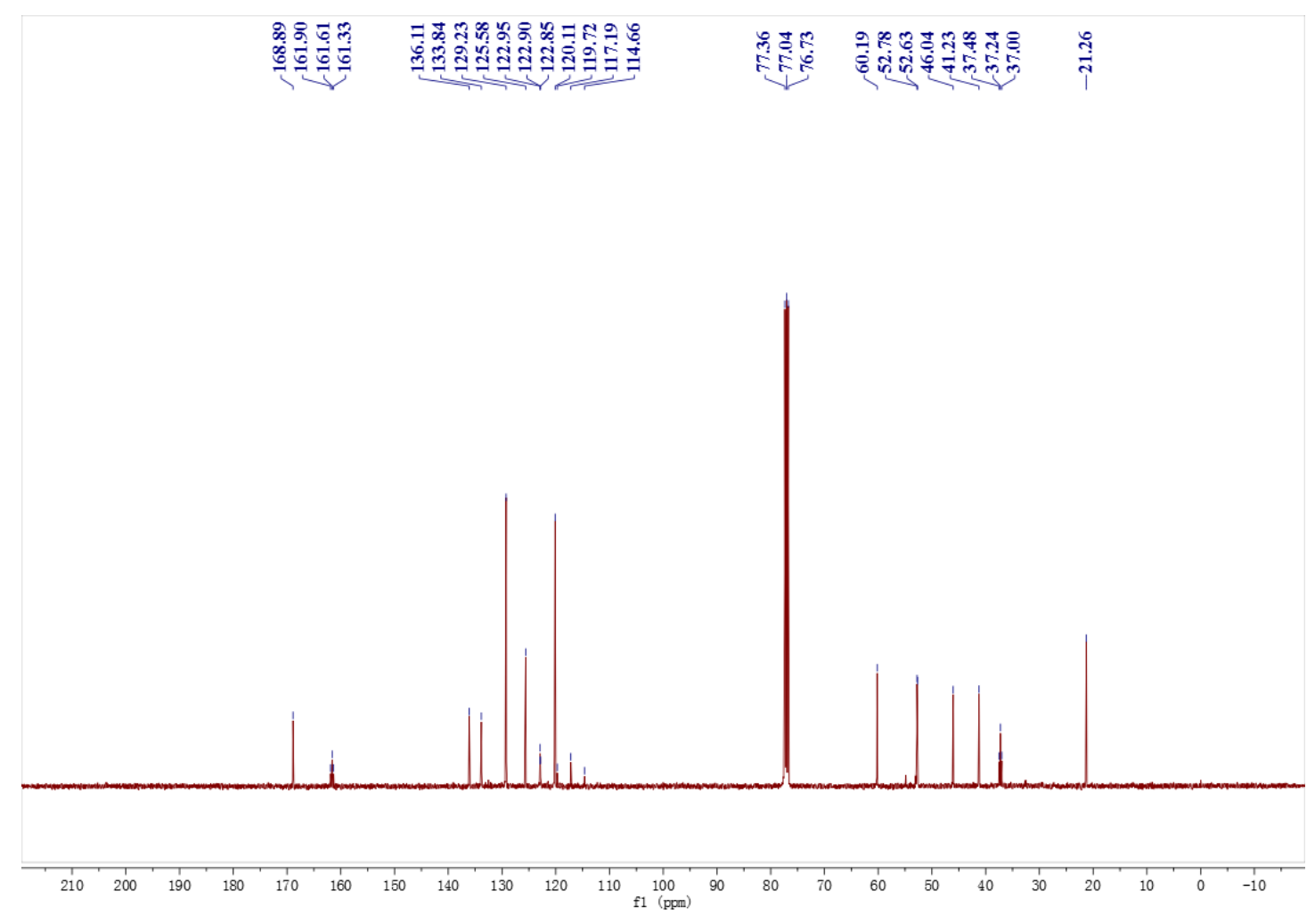




\section{X-ray crystallographic data of compound}

Crystal of compound 4a was prepared in a solvent mixture of $\mathrm{CHCl}_{3}$ and $n$-hexane (v/v $\left.=10 / 1\right)$. 4a $(\sim 50 \mathrm{mg})$ was firstly dissolved in $\mathrm{CHCl}_{3}(\sim 1 \mathrm{~mL})$ in a vial, then $n$-hexane $(\sim 0.1 \mathrm{~mL})$ was added dropwise to it. The vial was not fully screwed down and the sample was carefully setting in room temperature. The crystal was obtained in about $48 \mathrm{~h}$.

All the measurements were performed on a BRUKER Single Crystal X-Ray Diffractometer, Germany (model of the instrument -AXS D8 Quest System).

Specification: D8 QUEST, Photon 100 CMOS Detector, Horizontal Goniometer, Fixed Chi stage, Goniometer headmanual, Ceramic Tube KFF Mo-2K-90c, two pinhole collimator $(0.3 / 17 \mathrm{mrad}$, 0.6/17 mrad), Head turned by $90^{\circ}$, APEX2 w.SHELXTL S/W, Video microscope SCD, Cryostream- 700plus extended range low Temperature.

X-Ray crystallographic analysis of (E)-2,2-difluoro- $N$-phenyl-6-(phenylsulfonyl)hex-4-enamide 4a (2126957) showing the thermal ellipsoids at 30\% probability level.

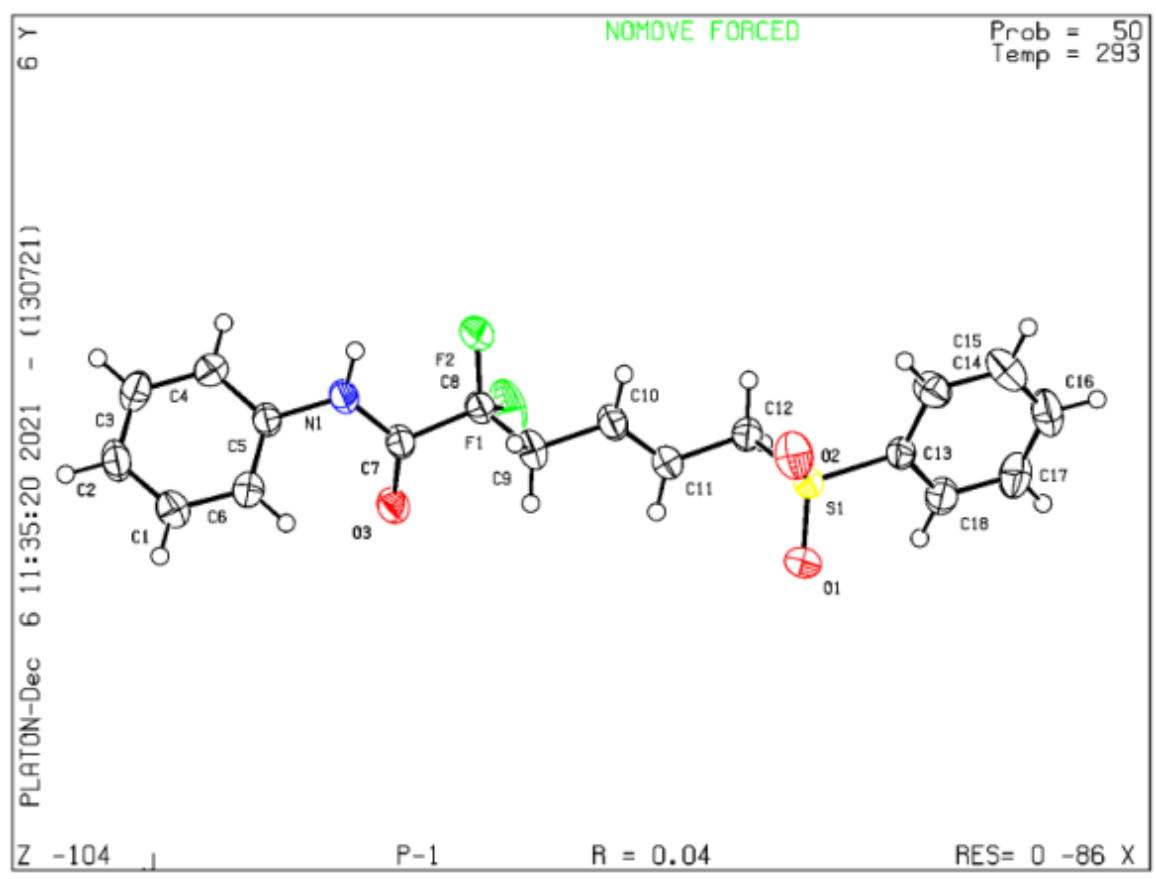




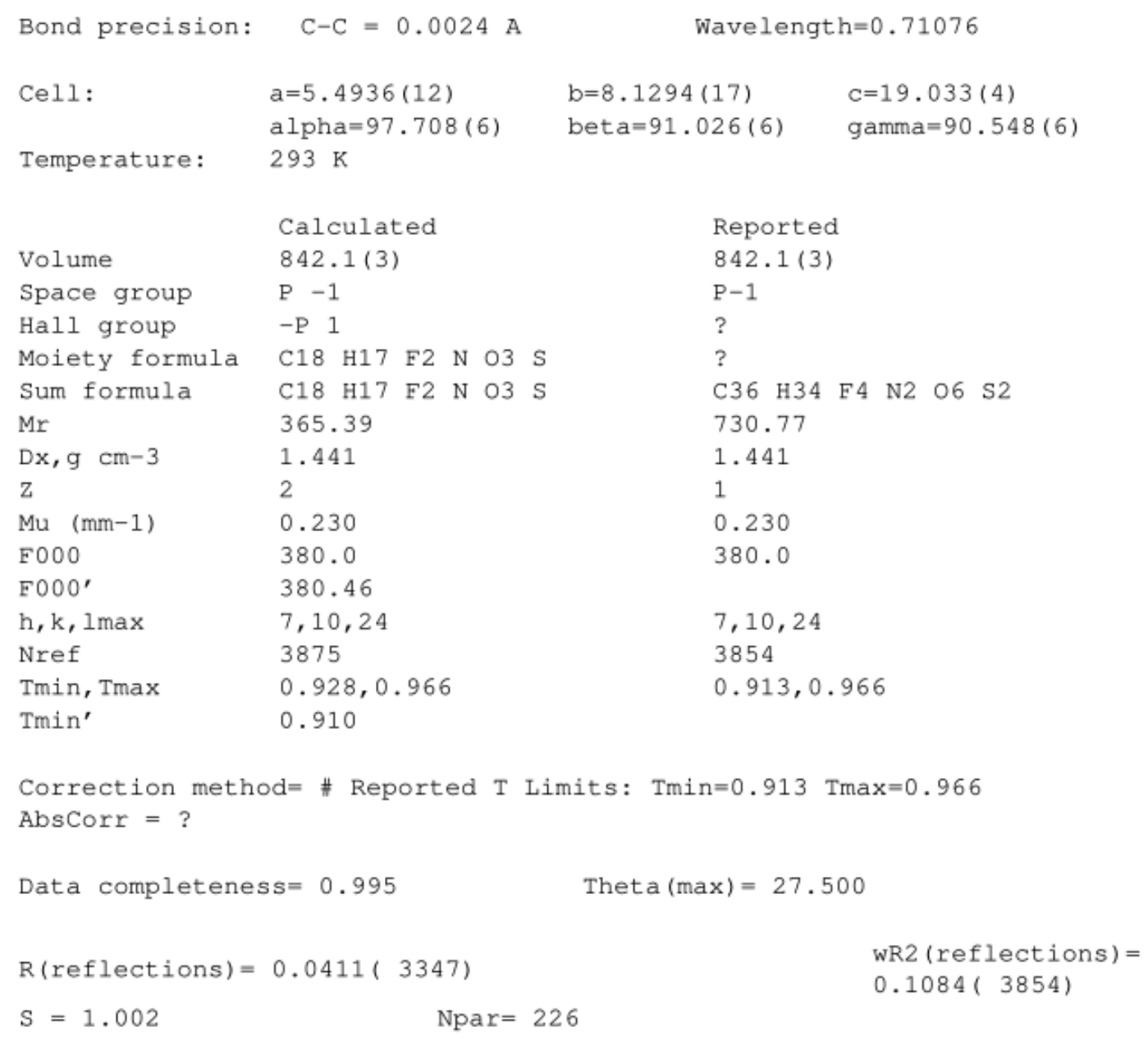

